\title{
Pontos parcialmente umbílicos em famílias a um parâmetro de hipersuperfícies imersas em $\mathbb{R}^{4}$
}

Débora Lopes da Silva

\section{TESE APRESENTADA}

$\mathrm{AO}$

Instituto DE MATEMÁtica E EstatísticA

DA

Universidade DE SÃo PAUlo

PARA

OBTENÇÃO DO TÍTULO

$\mathrm{DE}$

Doutor EM CiÊNCIAS

\author{
Programa: Matemática Aplicada \\ Orientador: Prof. Dr. Jorge Manuel Sotomayor Tello
}

Projeto financiado pelo CNPQ/CAPES

São Paulo, Novembro de 2012 


\title{
Pontos parcialmente umbílicos em famílias a um parâmetro de hipersuperfícies imersas em $\mathbb{R}^{4}$
}

\author{
Esta versão da tese contém as correções e alterações sugeridas \\ pela Comissão Julgadora durante a defesa da versão original do trabalho, \\ realizada em 09/11/2012. Uma cópia da versão original está disponível no \\ Instituto de Matemática e Estatística da Universidade de São Paulo.
}

Comissão Julgadora:

- Prof. Dr Jorge Manuel Sotomayor Tello - IME-USP

- Prof. Dr. Washington Luiz Marar - ICMC - USP

- Prof. Dr. Ronaldo Alves Garcia - UFG

- Prof. Dr. Antonio Carlos Asperti - IME - USP

- Prof. Dr. Fábio Scalco Dias - UNIFEI 


\section{Dedicatória}

Às mulheres da minha vida: a minha filha Ana Rosa e a minha mãe Ana Maria . 


\section{Agradecimentos}

À Deus, causa primária de todos as coisas, adradeço por ter me dado a oportunidade de alcançar meus objetivos, e por sua suprema sabedoria em guiar com cuidado os meus passos.

À minha filha, Ana Rosa, razão da minha existência, agradeço pela sua vida.

Agradeço a todos os amigos e colegas que contribuíram direta ou indiretamente com este trabalho. Em especial à Taty, Getúlio, Dy, Pricila, Gustavo, Gra, Juliano, Rose, Flausino e Gláucia pelas diversas maneiras de apoio, principalmente durante a gravidez: caronas, hospedagens e discussões matemáticas. Ao Humberto mais que um amigo, agradeço pela paciência, carinho, apoio e incentivo. Aos funcionários do IME por toda ajuda.

Agradeço, também, à todos os colegas professores do DMA-UFS pelo incentivo e apoio, em especial ao professor Paulo de Souza Rabelo, cujas iniciativas possibilitaram as condições necessárias para a conclusão deste trabalho.

Agradeço à Aryana, Francisco, Clara e João, família amiga, por todo o apoio com Ana Rosa.

Agradeço a madrinha, tia Terezinha, Tia Rosa e Gorki por todo incentivo, e pelo carinho e cuidado com Ana Rosa sempre que precisei me ausentar.

Ao meu orientador, professor Jorge Manuel Sotomayor Tello, agradeço muito pela paciência e atenção conferidas durante toda execução desta tese.

Ao professor Ronaldo Garcia, agradeço pelo grande apoio e pelas valiosas contribuições indispensáveis para a conclusão deste trabalho.

Aos demais membros da banca, Washington Luiz Marar, Fábio Scalco Dias e Antonio Carlos Asperti agradeço pelas sugestões.

Finalmente, aos meus pais, Argemiro e Ana, e aos meus irmãos Daniel, Hiram e Heber agradeço pelo amor, carinho e apoio sempre constante, sem os quais eu não estaria alcançando meus objetivos e seguindo a profissão que desejo. 


\section{Resumo}

Silva, D. L. Pontos parcialmente umbílicos em famílias a um parâmetro de hipersuperfícies imersas em $\mathbb{R}^{4}$. 2012. 187 f. Tese (Doutorado) - Instituto de Matemática e Estatística, Universidade de São Paulo, São Paulo, 2012.

Neste trabalho, estudamos as singularidades das folheações mutuamente ortogonais, numa variedade orientada $\mathbb{M}^{3}$ de dimensão 3, cujas folhas são as curvas integrais dos campos de direções de curvatura principal associadas a uma imersão $\alpha: \mathbb{M}^{3} \rightarrow \mathbb{R}^{4}$.

Damos aqui continuidade às contribuições de R. Garcia referente ao estudo das singularidades genéricas das folheações principais. Apresentamos as configurações principais numa vizinhança dos pontos parcialmente umbílicos de codimensão 1, ou seja, as singularidades das folheações principais que aparecem genericamente em famílias a 1 parâmetro de hipersuperfícies imersas em $\mathbb{R}^{4}$, e os diagramas de bifurcação pertinentes. Enfraquecendo a condição de genericidade, da maneira mais simples possível, encontramos oito tipos genéricos: $D_{1}^{1}, D_{2}^{1}, D_{3}^{1}, D_{13}^{1}, D_{1 h, p}^{1}, D_{1 h, n}^{1}, D_{p}^{1}$ e $D_{c}^{1}$, definidos ao longo do trabalho.

Nesta tese consubstanciamos matematicamente a seguinte conclusão: As singularidades das folheações principais, que aparecem genericamente em famílias a 1 parâmetro de hipersuperfícies imersas em $\mathbb{R}^{4}$, são os pontos parcialmente umbílicos $D_{1}^{1}, D_{2}^{1}, D_{3}^{1}$, $D_{13}^{1}, D_{1 h, p}^{1}, D_{1 h, n}^{1}, D_{p}^{1}$ e $D_{c}^{1}$, cujas definições e propriedades serão apresentados aqui. A parte central desta tese é estabelecer, analítica e geometricamente, a configuração principal destes pontos incluindo seus diagramas de bifurcação.

Palavras-chave: pontos parcialmente umbílicos, configuração principal, bifurcação. 


\section{Abstract}

\section{Silva, D. L. Partially Umbilic Points in One-parameter Families of Hypersur-}

faces Immersed in $\mathbb{R}^{4}$. 2012. 187 f. Tese (Doutorado) - Instituto de Matemática e Estatística, Universidade de São Paulo, São Paulo, 2012.

In this work we study the mutually ortogonal foliations, in oriented three dimensional manifolds $\mathbb{M}^{3}$, whose leaves are the integral curves of the principal curvature direction fields associated to immersions $\alpha: \mathbb{M}^{3} \rightarrow \mathbb{R}^{4}$. We focus on behavior of these foliation around singularities. Here we extend the contributions of R. Garcia concerning the study of generic singularities. To this end we establish the principal configurations in a neighborhood of partially umbilic points of codimension one. These are the singularities which appear generically in one parameter families of hypersurfaces and give their bifurcation diagrams. We express the condition of genericity by minimally weakening those given by R. Garcia and by adding instead new higher order ones. This procedure leads to the novel generic types: $D_{1}^{1}, D_{2}^{1}, D_{3}^{1}, D_{13}^{1}, D_{1 h, p}^{1}, D_{1 h, n}^{1}$, $D_{p}^{1}$ and $D_{c}^{1}$, studied in this work. The central part of this thesis is to establish, analitically and geometrically, the local principal configurations at these points, including their bifurcations diagrams.

Keywords: partially umbilic point, principal configuration, Bifurcation. 


\section{Conteúdo}

$\begin{array}{ll}\text { Introdução } & 13\end{array}$

1 Levantamento de Lie-Cartan e os Pontos Parcialmente Umbílicos 15

1.1 Preliminares . . . . . . . . . . . . . . . . . . 15

1.2 Levantamento de Lie-Cartan . . . . . . . . . . . . . . . . . . . 23

1.3 Apêndice: Cálculos até ordem 4 . . . . . . . . . . . . . . . 30

1.3.1 Coeficientes das formas fundamentais na carta de Monge reduzida 30

1.3.2 Expressões das formas fundamentais restritas ao campo de planos ortogonais à direção principal regular . . . . . . . . . . . . . . 34

1.3.3 Expressão de $L_{r}, M_{r}$ e $N_{r}$, até segunda ordem, na carta de Monge reduzida. . . . . . . . . . . . . . . . . 38

2 Estrutura das linhas de curvatura numa vizinhança de um ponto parcialmente umbílico Darbouxiano e semi-Darbouxiano 39

2.1 Preliminares . . . . . . . . . . . . . . . . . . . . . . . . . . 39

2.2 Pontos Parcialmente Umbílicos Darbouxianos . . . . . . . . . . . . . . 42

2.3 Curva Parcialmente Umbílica em torno de um ponto do tipo $D_{23}$. . . . 60

2.4 Linhas de curvatura em torno de um ponto do tipo $D_{12} \ldots \ldots$. . . . 73

2.5 Apêndice . . . . . . . . . . . . . . . . . . . . . . . . . 83

3 Pontos Parcialmente Umbílicos $D_{1}^{1}, D_{2}^{1}, D_{3}^{1}, D_{13}^{1}, D_{1 h, p}^{1}$ e $D_{1 h, n}^{1} \quad 86$

3.1 Linhas de Curvatura numa Vizinhança de um Ponto $D_{1}^{1}$. . . . . . . . 88

3.2 Linhas de Curvatura numa Vizinhança dos Pontos Parcialmente Umbílicos dos Tipos $D_{2}^{1}$ e $D_{3}^{1} \ldots \ldots \ldots 7$

3.3 Linhas de Curvatura numa Vizinhança de um Ponto $D_{13}^{1}$. . . . . . . . 104

3.4 Linhas de Curvatura numa Vizinhança de um Ponto $D_{1 h, p}^{1}$ e $D_{1 h, n}^{1}$. . . 122 
4 Pontos Parcialmente Umbílicos Críticos: $D_{p}^{1}$ e $D_{c}^{1} \quad 129$

4.1 Preliminares . . . . . . . . . . . . . . . . . . . 129

4.2 Linhas de curvatura numa vizinhança de um ponto parcialmente um-

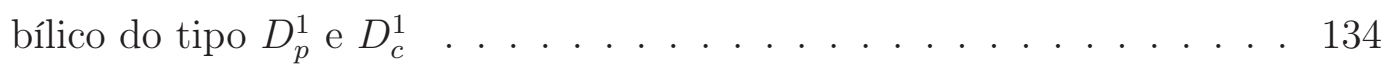

5 Teorema de Genericidade 143

6 Diagramas de Bifurcação dos Pontos Parcialmente Umbílicos $D_{1}^{1}, D_{2}^{1}$, $D_{3}^{1}, D_{13}^{1}, D_{1 h, n}^{1}, D_{1 h, p}^{1}, D_{p}^{1}$ e $D_{c}^{1} \quad 148$

6.1 Preliminares . . . . . . . . . . . . . . . . . . . . 148

6.2 Diagrama de Bifurcação de um Ponto Parcialmente Umbílico do tipo $D_{1}^{1} 151$

6.3 Diagramas de Bifurcação dos Pontos Parcialmente Umbílicos $D_{2}^{1}$ e $D_{3}^{1}$. 158

6.4 Diagrama de Bifurcação de um Ponto Parcialmente Umbílico do tipo $D_{13}^{1} 164$

6.5 Diagramas de Bifurcação dos Pontos Parcialmente Umbílicos $D_{1 h, p}^{1}$ e $D_{1 h, n}^{1} 171$

6.6 Diagramas de Bifurcação dos Pontos Parcialmente Umbílicos $D_{p}^{1}$ e $D_{c}^{1}$. 176

$\begin{array}{lll}7 & \text { Conclusões } & 183\end{array}$

$\begin{array}{lr}\text { Bibliografia } & 184\end{array}$ 


\section{Lista de Figuras}

1.1 Comportamento de $\mathcal{F}_{1}(\alpha)$ numa vizinhança de uma curva parcialmente umbílica Darbouxiana $c(\alpha)$ do tipo $D_{i}$, veja seção 2.2 do capítulo 2 . O mesmo acontece para a folheação $\mathcal{F}_{2}(\alpha)$. A folheação $\mathcal{F}_{3}(\alpha)$ é regular. .

1.2 Comportamento de $\mathcal{F}_{2}(\alpha)$ numa vizinhança de uma linha parcialmente umbílica contendo um ponto do tipo $D_{12}$ (esquerda), e de $\mathcal{F}_{1}(\alpha)$ e $\mathcal{F}_{2}(\alpha)$ numa vizinhança de uma linha parcialmente umbílica contendo um ponto do tipo $D_{23}$ (centro e direita) . . . . . . . . . . . . . 22

1.3 Contato da curva parcialmente umbílica com o plano umbílico. . . . . 27

2.1 Setor Parabólico . . . . . . . . . . . . . . . . . . . . . . . . . 41

2.2 Setor Hiperbólico . . . . . . . . . . . . . . . . . . . . . . . . . . . 41

2.3 Comportamento de $\mathcal{F}_{1}(\alpha)$ e $\mathcal{F}_{2}(\alpha)$ numa vizinhança de uma curva parcialmente umbílica Darbouxiana $c$ do tipo $D_{1}$. As folheações são unidimensionais.

2.4 Comportamento de $\mathcal{F}_{1}(\alpha)$ e $\mathcal{F}_{2}(\alpha)$ numa vizinhança de uma curva parcialmente umbílica Darbouxiana $c$ do tipo $D_{2}$. As folheações são unidimensionais. . . . . . . . . . . . . . . . . . . . 44 44

2.5 Comportamento de $\mathcal{F}_{1}(\alpha)$ e $\mathcal{F}_{2}(\alpha)$ numa vizinhança de uma curva parcialmente umbílica Darbouxiana $c$ do tipo $D_{3}$. As folheações são unidimensionais. . . . . . . . . . . . . . . . . . . 4 45

2.6 Projeção para o caso $D_{1} \ldots \ldots \ldots \ldots$. . . . . . . . . . 56

2.7 Projeção para o caso $D_{2} \operatorname{com} \frac{a}{b}<2 \ldots \ldots \ldots$. . . . . . . . 58

2.8 Projeção para o caso $D_{3} \ldots \ldots \ldots \ldots$

2.9 A figura do meio representa a configuração principal de $\alpha$ numa vizinhança de um ponto do tipo $D_{23}$, e as das laterais representam cada folheação $\mathcal{F}_{1}$ e $\mathcal{F}_{2}$ separadamente 
2.10 Equilíbrios de $X$ associados a um ponto parcialmente umbílico do tipo $D_{23} \ldots \ldots \ldots \ldots \ldots \ldots \ldots \ldots$

2.11 Retrato de fase do campo de Lie-Cartan restrito a hipersuperfície de Lie-Cartan, numa vizinhança da curva de equilíbrios $\beta_{1} \ldots \ldots 66$

2.12 Retrato de fase de $\left.Y_{r}\right|_{W^{c}} \ldots \ldots \ldots \ldots \ldots \ldots \ldots$

2.13 Seções da Hipersuperfície de Lie-Cartan e suas projeções na hipersuperfície imersa em $\mathbb{R}^{4}$.

2.14 Projeção das curvas integrais de $X_{\mathcal{L}}$ na hipersuperfície imersa em $\mathbb{R}^{4}$. Observamos que, numa vizinhança de $P_{2}(0)=\frac{c+\sqrt{c^{2}+4 b^{2}}}{2 b}$ e de $P_{3}(0)=$ $\frac{c-\sqrt{c^{2}+4 b^{2}}}{2 b}$, podemos escrever a hipersuperfície de Lie-Cartan da forma $u_{3}=u_{3}\left(u_{1}, u_{2}, P\right)$ (veja a equação $(2.27)$ ) e, numa vizinhaça de $P_{1}(0)=0$, a hipersuperfície de Lie-Cartan é dada por $u_{2}=u_{2}\left(u_{1}, u_{3}, P\right)$

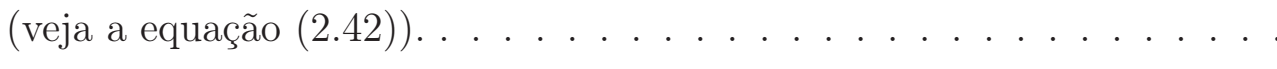

2.15 Variedades invariantes estratificadas numa vizinhança de um ponto do tipo $D_{23} \ldots \ldots \ldots \ldots \ldots \ldots \ldots \ldots \ldots$

2.16 Configuração Principal numa vizinhança de um ponto parcialmente umbílico do tipo $D_{12} \ldots \ldots \ldots$. . . . . . . . . . . . . . . . 74

2.17 Curvas $\beta_{1}$ e $\beta_{2}$ no plano $u_{3}$ P. . . . . . . . . . . . . . . 77

2.18 Equilíbrios de $X$ associados a um ponto parcialmente umbílico do tipo

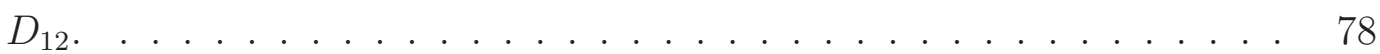

2.19 Retrato de fase de $X$, restrito à hipersuperfície de Lie-Cartan, numa vizinhança da origem. . . . . . . . . . . . . . . . 79

2.20 Retrato de fase de $X$ restrito a $W$ no ponto $\beta_{2}(0) \ldots \ldots$. . . . . . . . 82

2.21 Resolução de Lie-Cartan para um ponto parcialmente umbílico do tipo

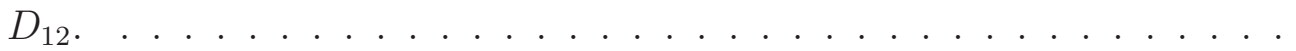

2.22 Variedades invariantes estratificadas numa vizinhança de um ponto do tipo $D_{12} \ldots \ldots \ldots \ldots \ldots \ldots \ldots \ldots \ldots$

3.1 Retrato de fase de $X_{c} \ldots \ldots \ldots \ldots \ldots$. . . . . . . . . . . 95

3.2 Retrato de fase de $X_{J} \ldots \ldots \ldots \ldots$. . . . . . . . . . 96

3.3 Retrato de fase de $X_{\mathcal{L}}$ restrito a variedade central para $\tilde{\chi}>0$ (esquerda) e $\tilde{\chi}<0$ (direita) . . . . . . . . . . . . . . . . . . . . . . 102

3.4 Retrato de fase de $X_{\mathcal{L}}$ numa vizinhança da curva de equilíbrios para $\tilde{\chi}>0$ (esquerda) e $\tilde{\chi}<0$ (direita). 
3.5 Projeção das curvas integrais do campo de Lie-Cartan na imersão $\alpha$, quando esta possui um ponto parcialmente umbílico do tipo $D_{3}^{1}$ (esquerda) ou $D_{2}^{1}$ (direita). . . . . . . . . . . . . . . . . . . . . 104

3.6 Linhas de curvatura numa vizinhança de um ponto do tipo $D_{13}^{1}$. . . . . 106

3.7 Retrato de fase de $X_{\mathcal{L}}$ restrito a variedade $W \ldots \ldots \ldots$. . . . . . 113

3.8 Variedade W. . . . . . . . . . . . . . . . . . . . . . . . . . . . . . . . . 114

3.9 Nesta figura, a curva vermelha representa a curva de equilíbrios. . . . . 115

3.10 Retrato de fase de $\tilde{X}$ próximo a esfera. . . . . . . . . . . . . . . . . 118

3.11 Retrato de fase de $X$ numa vizinhança da esfera, parte superior. . . . . 119

3.12 Ilustração dos resultados obtidos nesta seção. . . . . . . . . . . . . . . . 121

3.13 Linhas de curvatura numa vizinhança de um ponto do tipo $D_{1 h, n}^{1}$. . . . 124

3.14 Linhas de curvatura numa vizinhança de um ponto do tipo $D_{1 h, p}^{1}$. . . 125

3.15 Comportamento de $X_{\mathcal{L}}$ restrito a variedade central quando $\tilde{\chi}_{2}<0$. . 127

3.16 Comportamento de $X_{\mathcal{L}}$ restrito a variedade central quando $\tilde{\chi}_{2}>0$. . 127

3.17 Comportamento de $X_{\mathcal{L}}$ numa vizinhança da origem quando $\tilde{\chi}_{2}<0$. . 128

3.18 Comportamento de $X_{\mathcal{L}}$ numa vizinhança da origem quando $\tilde{\chi}_{2}>0 \ldots 128$

4.1 Configuração principal numa vizinhança de um ponto parcialmente um-

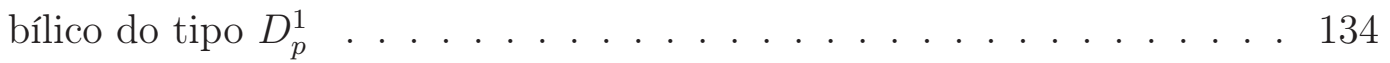

4.2 Configuração principal numa vizinhança de um ponto parcialmente umbílico do tipo $D_{c}^{1} \ldots \ldots \ldots \ldots$. . . . . . . . . . . . . . 135

4.3 Comportamento de $\left.X_{\mathcal{L}}\right|_{W^{c}}$ quando $\zeta<0 \ldots \ldots \ldots \ldots \ldots$

4.4 Comportamento de $\left.X_{\mathcal{L}}\right|_{W^{c}}$ quando $\zeta>0 \ldots \ldots \ldots \ldots \ldots$

6.1 Bifurcação de uma curva parcialmente umbílica que contém um ponto do tipo $D_{1}^{1}$. . . . . . . . . . . . . . . . . . . . . . . . . . 153

6.2 Resolução dos pontos parcialmente umbílicos de numa família a um parâmetro de hipersuperfícies, para o caso $D_{1}^{1}$. . . . . . . . . . . 157

6.3 Bifurcação de uma linha parcialmente umbílica que contém um ponto do tipo $D_{2}^{1}(\tilde{\chi}<0) \ldots \ldots \ldots \ldots$. . . . . . . . . . . . . 159

6.4 Bifurcação de uma linha parcialmente umbílica que contém um ponto do tipo $D_{3}^{1}(\tilde{\chi}>0) \ldots \ldots \ldots$. . . . . . . . . . . . . 160

6.5 Conjunto parcialmente umbílico da família $\alpha_{\lambda}$ nas proximidades de um ponto do tipo $D_{c}^{1} \ldots \ldots \ldots \ldots$. . . . . . . . . . . . . . . 164 
6.6 Bifurcação de uma linha parcialmente umbílica que contém um ponto do tipo $D_{13}^{1} \ldots \ldots \ldots \ldots \ldots$. . . . . . . . . . . . . . . 166

6.7 Conjunto parcialmente umbílico de uma família a 1 parâmetro associada a um ponto do tipo $D_{13}^{1}$. . . . . . . . . . . . . . . . . . . . . . . . . 169

6.8 Bifurcação de uma linha parcialmente umbílica que contém um ponto do tipo $D_{1 h, p}^{1} \ldots \ldots \ldots \ldots$. . . . . . . . . . . . . . 172

6.9 Bifurcação de uma linha parcialmente umbílica que contém um ponto do tipo $D_{1 h, n}^{1}$. . . . . . . . . . . . . . . . . . . . . . . . . . . . . . . 173

6.10 Bifurcação de uma linha parcialmente umbílica que contém um ponto do tipo $D_{p}^{1}$. . . . . . . . . . . . . . . . . . . . . . . . . . . 177

6.11 Bifurcação de uma linha parcialmente umbílica que contém um ponto do tipo $D_{c}^{1}$. . . . . . . . . . . . . . . . . . . . . . . 178

6.12 Conjunto parcialmente umbílico da família $\alpha_{\lambda}$ nas proximidades de um ponto do tipo $D_{p}^{1} \ldots \ldots \ldots$. . . . . . . . . . . . . . . 181

6.13 Conjunto parcialmente umbílico da família $\alpha_{\lambda}$ nas proximidades de um ponto do tipo $D_{c}^{1} \ldots \ldots \ldots \ldots$. . . . . . . . . . . . . . . . . 182 


\section{Introdução}

Para uma variedade de dimensão $n$ imersa em $\mathbb{R}^{n+1}$ são associadas $n$ folheações de dimensão 1, mutuamente ortogonais, com singularidades. As folhas são as curvas integrais dos campos de direções segundo as quais a curvatura normal é crítica. As curvaturas normais correspondentes são chamadas curvaturas principais e as folhas pertinentes de linhas de curvatura principal. Assim, para $n=2$ teremos duas curvaturas principais $k_{1}$ (mínima) $\leq k_{2}$ (máxima), para $n=3$ teremos três curvaturas principais $k_{1}$ (mínima) $\leq k_{2}($ intermediária $) \leq k_{3}$ (máxima), etc. Trataremos o caso $n=3$.

Para superfícies imersas em $\mathbb{R}^{3}$ as singularidades das folheações principais ocorrem onde as duas curvaturas principais são iguais, ou seja, nos pontos umbílicos. O estudo das linhas de curvatura, numa vizinhança de tais pontos, remonta aos trabalhos de Monge, Dupin, Darboux, entre outros. No contexto de sistemas dinâmicos e teoria das folheações, tal estudo foi iniciado em 1982 por Gutierrez e Sotomayor, ver por exemplo [12], [13], [14], [8] e [10]. Darboux [2] determinou o comportamento das folheações principais numa vizinhança de um ponto umbílico genérico no caso analítico, estes pontos são conhecidos como pontos umbílicos Darbouxianos e aparecem em [12] no contexto de estabilidade estrutural das linhas de curvatura principal numa versão para classe $C^{4}$.

Para hipersuperfícies imersas em $\mathbb{R}^{4}$ teremos dois tipos de singularidades: um tipo ocorre nos pontos umbílicos da imersão e o outro nos pontos parcialmente umbílicos, onde duas das curvaturas principais são iguais. O estudo das folheações principais numa vizinhança de um ponto parcialmente umbílico foi iniciado por R. Garcia em [6], onde ele caracterizou os tipos genéricos.

O objetivo deste trabalho consiste no estudo dos pontos parcialmente umbílicos que aparecem genericamente em famílias a um parâmetro de hipersuperfícies imersas em $\mathbb{R}^{4}$. Abordaremos as configurações principais em torno dos ditos pontos e suas bifurcações. Para o caso de superfícies em $\mathbb{R}^{3}$ tal estudo foi desenvolvido em [8] por 
Garcia, Gutierrez e Sotomayor.

No capítulo 1 apresentamos o levantamento de Lie-Cartan adaptado para o estudo das linhas de curvatura próximas a um ponto parcialmente umbílico. Para a análise dos pontos umbílicos em superfícies isto foi feito em [1] por Bruce e Fidal. Ver também $[8]$.

No capítulo 2, como aplicação preliminar do levantamento de Lie-Cartan descrito no capítulo 1, daremos uma nova demonstração dos resultados de R. Garcia referentes à estrutura das linhas de curvatura principal numa vizinhança de uma curva regular formada por pontos parcialmente umbílicos Darbouxianos e em torno dos pontos SemiDarbouxianos. Ver definições 1 e 2 no capítulo 1.

No capítulo 3 , definimos os pontos parcialmente umbílicos regulares $D_{1}^{1}, D_{2}^{1}$, $D_{3}^{1}, D_{1 h, p}^{1}, D_{1 h, n}^{1}$ e $D_{13}^{1}$ e analisamos as folheações principais numa vizinhança de tais pontos. Estes pontos aparecem isoladamente ao longo de curvas regulares de pontos parcialmente umbílicos e têm estreita relação com os pontos umbílicos de codimensão 2, estudados por Garcia e Sotomayor em [9].

No capítulo 4, definimos os pontos parcialmente umbílicos para o caso crítico, $D_{p}^{1}$ e $D_{c}^{1}$, e estudamos as folheações principais numa vizinhança de tais pontos.

No capítulo 5 definimos a estratificação canônica do espaço dos 5-jatos das imersões, $\alpha$, de $\mathbb{M}^{3}$ em $\mathbb{R}^{4}$ e, usando o teorema de transversalidade de Thom [17], mostraremos que, genericamente, as singularidades das folheações principais que aparecem em famílias a 1 parâmetro de hipersuperfícies são exatamente os pontos parcialmente umbílicos $D_{1}^{1}$, $D_{2}^{1}, D_{3}^{1}, D_{13}^{1}, D_{1 h, p}^{1}, D_{1 h, n}^{1}, D_{p}^{1}$ e $D_{c}^{1}$, estudados nos capítulos 3 e 4 . Neste sentido, estes pontos serão ditos de codimensão 1 .

No capítulo 6 estudaremos as mudanças qualitativas -bifurcações- que ocorrem nas configurações principais dos pontos parcialmente umbílicos $D_{1}^{1}, D_{2}^{1}, D_{3}^{1}, D_{13}^{1}, D_{1 h, p}^{1}$, $D_{1 h, n}^{1}, D_{p}^{1}$ e $D_{c}^{1}$.

Um resumo dos resultados deste trabalho é apresentado no capítulo 7. 


\section{Capítulo 1}

\section{Levantamento de Lie-Cartan e os Pontos Parcialmente Umbílicos}

\subsection{Preliminares}

Seja $\mathbb{M}^{3}$ uma variedade compacta de dimensão 3 , orientada e de classe $C^{k}, k \geq 5$. Uma imersão $\alpha: \mathbb{M}^{3} \rightarrow \mathbb{R}^{4}$ é uma aplicação tal que $D \alpha_{p}: T \mathbb{M}_{p}^{3} \rightarrow \mathbb{R}^{4}$ é injetiva para todo $p \in \mathbb{M}^{3}$. Denotemos por $\mathcal{I}^{k, s}=\mathcal{I}^{k, s}\left(\mathbb{M}^{3}, \mathbb{R}^{4}\right)(s \leq k)$ o conjunto das imersões de $\mathbb{M}^{3}$ em $\mathbb{R}^{4}$ de classe $C^{k}$, munido com a topologia $C^{s}$. Associada a cada $\alpha \in \mathcal{I}^{k}$, definimos a normal à imersão $\alpha, N_{\alpha}: \mathbb{M}^{3} \rightarrow \mathbb{R}^{4}$, por

$$
N_{\alpha}=\frac{\alpha_{1} \wedge \alpha_{2} \wedge \alpha_{3}}{\left|\alpha_{1} \wedge \alpha_{2} \wedge \alpha_{3}\right|}
$$

onde $\wedge$ denota o produto vetorial em $\mathbb{R}^{4}$ cuja orientação é dada por este produto, $\alpha_{1}=\frac{\partial \alpha}{\partial u_{1}}, \alpha_{2}=\frac{\partial \alpha}{\partial u_{2}}, \alpha_{3}=\frac{\partial \alpha}{\partial u_{3}}$ onde $\left(u_{1}, u_{2}, u_{3}\right):\left(\mathbb{M}^{3}, p\right) \rightarrow\left(\mathbb{R}^{3}, 0\right)$ é uma carta positiva em torno de $p$, e $\mid=<,>^{1 / 2}$ é a norma euclidiana em $\mathbb{R}^{4}$. As Formas Fundamentais de $\alpha$ em um ponto $p$ de $\mathbb{M}^{3}$ são

$$
\begin{aligned}
I_{\alpha} & =\sum_{i, j=1}^{3} g_{i j}(p) d u_{i} d u_{j}=v G v^{T}, \\
I I_{\alpha} & =\sum_{i, j=1}^{3} \lambda_{i j}(p) d u_{i} d u_{j}=v \Lambda v^{T},
\end{aligned}
$$

onde $g_{i j}=\left\langle\frac{\partial \alpha}{\partial u_{i}}, \frac{\partial \alpha}{\partial u_{j}}\right\rangle, \lambda_{i j}=\left\langle\frac{\partial^{2} \alpha}{\partial u_{i} \partial u_{j}}, N_{\alpha}\right\rangle, G=\left[g_{i j}\right]_{3 \times 3}, \Lambda=\left[\lambda_{i j}\right]_{3 \times 3}$ e $v=$ $\left(d u_{1}, d u_{2}, d u_{3}\right)$. Observamos que $g_{i j}=g_{j i}$ e $\lambda_{i j}=\lambda_{j i}$. 
A curvatura normal de $\alpha$ no ponto $p$ e numa direção $v=\left(d u_{1}, d u_{2}, d u_{3}\right)$ é definida por $k_{n}(p, v)=\frac{I I_{\alpha}}{I_{\alpha}}$. As direções onde $k_{n}(p, \cdot)$ assume valores críticos são chamadas $d i$ reções principais. Os valores de $k_{n}$ nestas direções são chamados curvaturas principais, as quais denotaremos por $k_{1}(p) \leq k_{2}(p) \leq k_{3}(p)$.

Observamos que as direções onde $k_{n}(p, \cdot)$ assume valor crítico, restrito à condição $I_{\alpha}=1$, são dadas pelo método dos multiplicadores de Lagrange com multiplicador $k$, resolvendo a equação

$$
\nabla I I_{\alpha}=k \nabla I_{\alpha}
$$

Neste caso, $k$ é uma das curvaturas principais. Disto, tais curvaturas são definidas pela equação

$$
\operatorname{det}(\Lambda-k G)=0
$$

Da equação (1.1) resulta que as direções principais são as soluções do seguinte sistema de equações lineares:

$$
\left\{\begin{array}{l}
\left(\lambda_{11}-k_{i} g_{11}\right) d u_{1}+\left(\lambda_{12}-k_{i} g_{12}\right) d u_{2}+\left(\lambda_{13}-k_{i} g_{13}\right) d u_{3}=0 \\
\left(\lambda_{12}-k_{i} g_{12}\right) d u_{1}+\left(\lambda_{22}-k_{i} g_{22}\right) d u_{2}+\left(\lambda_{23}-k_{i} g_{23}\right) d u_{3}=0 \\
\left(\lambda_{13}-k_{i} g_{13}\right) d u_{1}+\left(\lambda_{23}-k_{i} g_{23}\right) d u_{2}+\left(\lambda_{33}-k_{i} g_{33}\right) d u_{3}=0
\end{array}\right.
$$

onde $k_{i}(i=1,2,3)$ são as curvaturas principais.

Associadas às direções principais, dadas pelo sistema (1.3), temos três campos de linhas chamados campos de linhas principais. Dois tipos de singularidades, isto é, pontos onde estes não estão bem definidos, podem aparecer para estes campos. Estas são os pontos umbílicos onde as três curvaturas principais coincidem, e os pontos parcialmente umbilicos, onde apenas duas delas coincidem. Definimos $\mathcal{U}(\alpha)=\{p \in$ $\left.\mathbb{M}^{3}: k_{1}(p)=k_{2}(p)=k_{3}(p)\right\}, \mathcal{P}_{12}(\alpha)=\left\{p \in \mathbb{M}^{3}: k_{1}(p)=k_{2}(p) \neq k_{3}(p)\right\}, \mathcal{P}_{23}(\alpha)=$ $\left\{p \in \mathbb{M}^{3}: k_{1}(p) \neq k_{2}(p)=k_{3}(p)\right\}$, e $\mathcal{P}(\alpha)=\mathcal{P}_{12}(\alpha) \cup \mathcal{P}_{23}(\alpha)$. Os conjuntos $\mathcal{U}(\alpha)$ e $\mathcal{P}(\alpha)$ são chamados, respectivamente, conjunto umbílico e conjunto parcialmente umbílico da imersão $\alpha$.

Seja $k(p)=k_{1}(p)=k_{2}(p)$. Em torno de $p \in \mathcal{P}_{12}(\alpha)$, podemos escrever localmente a imersão $\alpha$ como gráfico de uma função definida no espaço tangente, ou seja, se $N(p)$ é o vetor normal à imersão em $p, e_{3}(p)$ é a direção principal associada a $k_{3}(p)$ e $\left\{e_{1}(p), e_{2}(p)\right\}$ é uma base ortonormal do auto-espaço de $D N(p)$ correspondente $^{1}$ a $-k(p)$, tal que $\left(e_{1} \wedge e_{2} \wedge e_{3}\right)(p)=N(p)$, então

$$
\alpha\left(u_{1}, u_{2}, u_{3}\right)=u_{1} e_{1}(p)+u_{2} e_{2}(p)+u_{3} e_{3}(p)+h\left(u_{1}, u_{2}, u_{3}\right) N(p)
$$

\footnotetext{
${ }^{1}$ Veja [15] para mais detalhes sobre a derivada da aplicação normal.
} 
onde

$$
\begin{aligned}
h\left(u_{1}, u_{2}, u_{3}\right) & =\frac{k}{2}\left(u_{1}^{2}+u_{2}^{2}\right)+\frac{k_{3}}{2} u_{3}^{2}+\frac{a}{6} u_{1}^{3}+\frac{b}{2} u_{1} u_{2}^{2}+\frac{c}{6} u_{2}^{3}+\frac{q_{003}}{6} u_{3}^{3} \\
& +\frac{q_{012}}{2} u_{2} u_{3}^{2}+q_{111} u_{1} u_{2} u_{3}+\frac{q_{021}}{2} u_{2}^{2} u_{3}+\frac{q_{102}}{2} u_{1} u_{3}^{2} \\
& +\frac{q_{201}}{2} u_{1}^{2} u_{3}+O(4) .
\end{aligned}
$$

Observe que nesta expressão de $h$ o termo $u_{1}^{2} u_{2}$ não aparece, isto pode ser obtido escolhendo adequadamente a base ortonormal $\left\{e_{1}(p), e_{2}(p)\right\}$. A carta dada em (1.4) é conhecida como carta de Monge. Quando escrevemos $\alpha$ como em (1.4) com $h$ da forma (1.5) (ou seja, sem o termo $u_{1}^{2} u_{2}$ ), dizemos que $\alpha$ está escrita na carta de Monge reduzida (por rotação).

Além da restrição feita, com uma homotetia e uma inversão podemos fazer $k_{3}=1$ e $k=0$.

Tais transformações não mudam a estrutura topológica das linhas de curvatura numa vizinhança de um ponto parcialmente umbílico.

Definição 1 [Ponto Parcialmente Umbílico Darbouxiano/ Sejam $p \in \mathbb{M}^{3}$ um ponto parcialmente umbílico tal que $k_{1}(p)=k_{2}(p)=k(p) \neq k_{3}(p)=1$ e $\left(u_{1}, u_{2}, u_{3}\right): \mathbb{M}^{3} \rightarrow \mathbb{R}^{3}$ a carta de Monge reduzida. O ponto p é chamado parcialmente umbílico Darbouxiano do tipo $D_{i}$ se satisfaz às condições $T$ ) e $D_{i}$ ) abaixo:

T) $b(b-a) \neq 0$;

$\left.D_{1}\right) \frac{a}{b}>\left(\frac{c}{2 b}\right)^{2}+2$;

D ) $1<\frac{a}{b}<\left(\frac{c}{2 b}\right)^{2}+2, a \neq 2 b$;

$\left.D_{3}\right) \frac{a}{b}<1$.

A definição acima independe da escolha da base $\left\{e_{1}(p), e_{2}(p)\right\}$. De fato, tomemos uma outra base ortonormal $\left\{\tilde{e}_{1}(p), \tilde{e}_{2}(p)\right\}$ do autoespaço, de $D N(p)$, associado à $-k(p)$ e satisfazendo $\left(\tilde{e}_{1} \wedge \tilde{e}_{2} \wedge e_{3}\right)(p)=N(p)$, com

$$
\alpha\left(\tilde{u}_{1}, \tilde{u}_{2}, u_{3}\right)=\tilde{u}_{1} \tilde{e}_{1}(p)+\tilde{u}_{2} \tilde{e}_{2}(p)+u_{3} e_{3}(p)+\tilde{h}\left(\tilde{u}_{1}, \tilde{u}_{2}, u_{3}\right) N(p)
$$


onde

$$
\begin{aligned}
\tilde{h}\left(\tilde{u}_{1}, \tilde{u}_{2}, u_{3}\right) & =\frac{k}{2}\left(\tilde{u}_{1}^{2}+\tilde{u}_{2}^{2}\right)+\frac{k_{3}}{2} u_{3}^{2}+\frac{1}{6} \tilde{a} \tilde{u}_{1}^{3}+\frac{1}{2} \tilde{d}_{1}^{2} \tilde{u}_{2}+\frac{1}{2} \tilde{b}_{1} \tilde{u}_{2}^{2}+\frac{1}{6} \tilde{c} \tilde{u}_{2}^{3} \\
& +\frac{1}{6} p_{003} u_{3}^{3}+\frac{1}{2} p_{012} \tilde{u}_{2} u_{3}^{2}+p_{111} \tilde{u}_{1} \tilde{u}_{2} u_{3}+\frac{1}{2} p_{021} \tilde{u}_{2}^{2} u_{3} \\
& +\frac{1}{2} p_{102} \tilde{u}_{1} u_{3}^{2}+\frac{1}{6} p_{201} \tilde{u}_{1}^{2} u_{3}+\tilde{O}(4) .
\end{aligned}
$$

Seja $\theta$ o ângulo entre $\tilde{e}_{1}$ e $e_{1}$ :

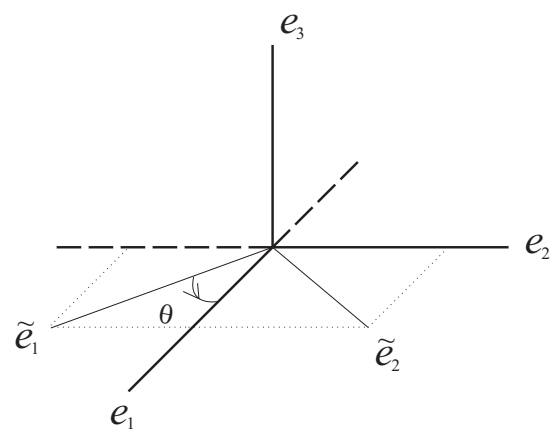

Então, as coordenadas $\left(u_{1}, u_{2}\right)$ e $\left(\tilde{u}_{1}, \tilde{u}_{2}\right)$ se relacionam por $u_{1}=\cos \theta \tilde{u}_{1}+\operatorname{sen} \theta \tilde{u}_{2}$, $u_{2}=-\operatorname{sen} \theta \tilde{u}_{1}+\cos \theta \tilde{u}_{2}$. Substituindo-as na expressão de $\tilde{h}$ acima obtemos que

$$
\tilde{d}=b \operatorname{sen}^{2} \theta+c \operatorname{sen} \theta \cos \theta+(a-2 b) \cos ^{2} \theta .
$$

Observemos que se $\cos \theta=0$ então $\tilde{d}=b \neq 0$. Assim, podemos supor que $\cos \theta \neq 0$. Escrevemos

$$
\tilde{d}=\tau \cos ^{3} \theta\left(b \tau^{2}+c \tau+a-2 b\right), \quad \tau=\frac{\operatorname{sen} \theta}{\cos \theta} .
$$

Para expressarmos as condições $\left.T), D_{1}\right), D_{2}$ ) e $D_{3}$ ) na definição 1 , com respeito à base $\left\{\tilde{e}_{1}(p), \tilde{e}_{2}(p)\right\}$, devemos ter que $\tilde{d}=0$. Assim, ou $\theta=0, \pi$ ou $\theta$ satisfaz $b \tau^{2}+c \tau+a-2 b=$ 0 . Se $\theta$ é 0 ou $\pi$, as condições $\left.\left.T), D_{1}\right), D_{2}\right)$ e $\left.D_{3}\right)$ são trivialmente satisfeitas para os coeficientes $\tilde{a}, \tilde{b}$ e $\tilde{c}$, já que $|\tilde{a}|=|a|,|\tilde{b}|=|b|$ e $|\tilde{c}|=|c|$. Se $\operatorname{sen} \theta \neq 0$ então, impondo a condição $b \tau^{2}+c \tau+a-2 b=0$, obtemos que

$$
\begin{aligned}
& \tilde{a}=\frac{-b \cos ^{2} \theta+b+a \cos ^{2} \theta}{\cos \theta} \\
& \tilde{b}=(-b+a) \cos \theta \\
& \tilde{c}=-\frac{-2 b \cos ^{2} \theta+2 \cos ^{2} \theta a-a}{\operatorname{sen} \theta}
\end{aligned}
$$

donde vemos que $\tilde{b}(\tilde{b}-\tilde{a})=b(b-a)$, implicando que a condição $T)$ é invariante por mudança de coordenadas. Com relação às condições $\left.\left.D_{1}\right), D_{2}\right)$ e $D_{3}$ ), temos o seguinte: 
- Se $\frac{a}{b}>\left(\frac{c}{2 b}\right)^{2}+2$ então a equação $b \tau^{2}+c \tau+a-2 b=0$ não tem raízes reais, logo $\theta$ é 0 ou $\pi$ e, portanto, $a= \pm \tilde{a}, b= \pm \tilde{b}$ e $c= \pm \tilde{c}$.

- Se $1<\frac{a}{b}<\left(\frac{c}{2 b}\right)^{2}+2, a \neq 2 b$ então

$$
\frac{\tilde{a}}{\tilde{b}}=\frac{\left(\cos ^{2} \theta\right) a+b-b \cos ^{2} \theta}{\cos ^{2} \theta(a-b)}=1+\frac{1}{\cos ^{2} \theta(a / b-1)}>1 \text { e } \neq 2,
$$

assim como

$$
\begin{aligned}
\left(\frac{\tilde{c}}{2 \tilde{b}}\right)^{2}-\frac{\tilde{a}}{\tilde{b}}+2 & =\frac{1}{4} \frac{a^{2}+4 b^{2}-4 a b}{\operatorname{sen}^{2} \theta(a-b)^{2} \cos ^{2} \theta} \\
& =\frac{1}{\operatorname{sen}^{2} \theta \cos ^{2} \theta(a / b-1)^{2}}\left(\left(\frac{c}{2 b}\right)^{2}-\frac{a}{b}+2\right)>0
\end{aligned}
$$

- E, finalmente, se a condição $D_{3}$ ) é válida então, por (1.7), teremos que $\frac{\tilde{a}}{\tilde{b}}<1$.

Assim, concluímos que a definição 1 independe da escolha da base do autoespaço, de $D N(p)$, associado a $-k$, que torna $d=0$, onde $d$ é o coeficiente do termo $u_{1}^{2} u_{2}$ na carta de Monge.

Em [7], Garcia mostrou que um ponto parcialmente umbílico satisfazendo a condição $T$ ) pertence a uma curva regular formada por pontos parcialmente umbílicos, isto será demonstrado aqui na proposição 1. Um arco regular de pontos parcialmente umbílicos do tipo $D_{i}(i=1,2,3)$ é chamado curva parcialmente umbílica Darbouxiana do tipo $D_{i}(i=1,2,3)$.

Ao longo de uma componente conexa de $\mathcal{P}(\alpha)$, é esperado que possam ocorrer transições entre os tipos $D_{i}, i=1,2,3$. As transições mais simples são as $D_{1}-D_{2}$ e $D_{2}-D_{3}$, que ocorrem em torno dos pontos parcialmente umbílicos Semi-Darbouxianos $D_{12}$ e $D_{23}$, que definiremos a seguir. Estes pontos têm estreita relação com os pontos umbílicos de codimensão 1 em superfícies, estudados por R. Garcia, C. Gutierrez e J. Sotomayor em [8].

Sejam $p \in \mathbb{M}^{3}$ tal que $k_{1}(p)=k_{2}(p)=k(p) \neq k_{3}(p)$ e $\left(u_{1}, u_{2}, u_{3}\right): \mathbb{M}^{3} \rightarrow \mathbb{R}^{3}$ a 
carta de Monge reduzida ( ver (1.4)), com $k_{3}(p)=1$, onde

$$
\begin{aligned}
h\left(u_{1}, u_{2}, u_{3}\right)= & \frac{k}{2}\left(u_{1}^{2}+u_{2}^{2}\right)+\frac{1}{2} u_{3}^{2}+\frac{1}{6} a u_{1}^{3}+\frac{1}{2} b u_{1} u_{2}^{2}+\frac{1}{6} c u_{2}^{3}+\frac{1}{6} q_{003} u_{3}^{3} \\
+ & \frac{1}{2} q_{012} u_{2} u_{3}^{2}+q_{111} u_{1} u_{2} u_{3}+\frac{1}{2} q_{021} u_{2}^{2} u_{3}+\frac{1}{2} q_{102} u_{1} u_{3}^{2} \\
& +\frac{1}{2} q_{201} u_{1}^{2} u_{3}+\frac{1}{24} A u_{1}^{4}+\frac{1}{6} B u_{1}^{3} u_{2}+\frac{1}{4} C u_{1}^{2} u_{2}^{2}+\frac{1}{6} D u_{1} u_{2}^{3} \\
& +\frac{1}{24} E u_{2}^{4}+\frac{1}{24} Q_{004} u_{3}^{4}+\frac{1}{6} Q_{013} u_{2} u_{3}^{3}+\frac{1}{6} Q_{103} u_{1} u_{3}^{3}+\frac{1}{4} Q_{022} u_{2}^{2} u_{3}^{2} \\
& +\frac{1}{4} Q_{202} u_{1}^{2} u_{3}^{2}+\frac{1}{2} Q_{112} u_{1} u_{2} u_{3}^{2}+\frac{1}{6} Q_{031} u_{2}^{3} u_{3}+\frac{1}{6} Q_{301} u_{1}^{3} u_{3} \\
& +\frac{1}{2} Q_{121} u_{1} u_{2}^{2} u_{3}+\frac{1}{2} Q_{211} u_{1}^{2} u_{2} u_{3}+O(5) .
\end{aligned}
$$

Definição 2 [Ponto Parcialmente Umbílico Semi-Darbouxiano $D_{23}$ ] O ponto p é chamado parcialmente umbílico Semi-Darbouxiano do tipo $D_{23}$ se:

$\left.D_{23}\right) \quad b=a \neq 0,-b\left(-q_{201}+q_{021}\right)-c q_{111} \neq 0 e$

$$
\chi=c B-\left(C-A+2 k^{3}\right) b+\frac{-2 q_{111}^{2} b+2 q_{201}^{2} b+2 q_{201} q_{111} c}{b(k-1)} \neq 0
$$

Definição 3 [Ponto Parcialmente Umbilico Semi-Darbouxiano $D_{12}$ / O ponto p é chamado parcialmente umbílico Semi-Darbouxiano do tipo $D_{12}$ se:

$\left.D_{12}\right) c b(b-a) \neq 0, \frac{a}{b}=2$ e $\chi_{2} \neq 0$. onde

$$
\begin{aligned}
\chi_{2} & =\frac{1}{27 b^{6}(k-1)}\left[q_{111} b C+q_{201} B b+2 q_{111} q_{201} b q_{021}+2 q_{111} q_{102} b^{2}+\right. \\
& +q_{111} B c-3 q_{201} q_{111}^{2} c-2 q_{111}^{3} b-Q_{211} b^{2}-3 q_{111} q_{201}^{2} b-B b q_{021}+ \\
& +q_{201} q_{012} b^{2}+\left(B b q_{021}+Q_{211} b^{2}-q_{201} B b-q_{111} B c-q_{111} b C\right) k- \\
& \left.-q_{111} b k^{3}+q_{111} b k^{4}\right] c^{3}
\end{aligned}
$$

Observação 10 não anulamento da constante $\chi_{2}$ garante a transição transversal $D_{1}-D_{2}$ ao longo da curva parcialmente umbílica que contém um ponto deste tipo, como mostraremos na seção 2.4 do capítulo 2. 
Observação 2 A estrutura das linhas de curvatura numa vizinhança de um ponto parcialmente umbilico onde $\frac{a}{b}=\left(\frac{c}{2 b}\right)^{2}+2$ é topologicamente equivalente à de um ponto do tipo $D_{12}$ (definição 3 acima).

Fora do conjunto $\mathcal{P}(\alpha) \cup \mathcal{U}(\alpha)$, os campos de linhas principais, denotados por $\mathcal{L}_{i}(\alpha)(i=1,2,3)$, são mutuamente ortogonais. As curvas integrais destes campos são chamadas linhas de curvatura principal (ou linhas principais) e as folheações resultantes $\mathcal{F}_{i}(\alpha)(i=1,2,3)$ são chamadas folheações principais.

A estrutura das linhas de curvatura, próximas a um arco parcialmente umbílico Darbouxiano e em torno de um ponto Semi-Darbouxiano, foi estudada por R. Garcia em [6]. (ver figuras 1.1 e 1.2)
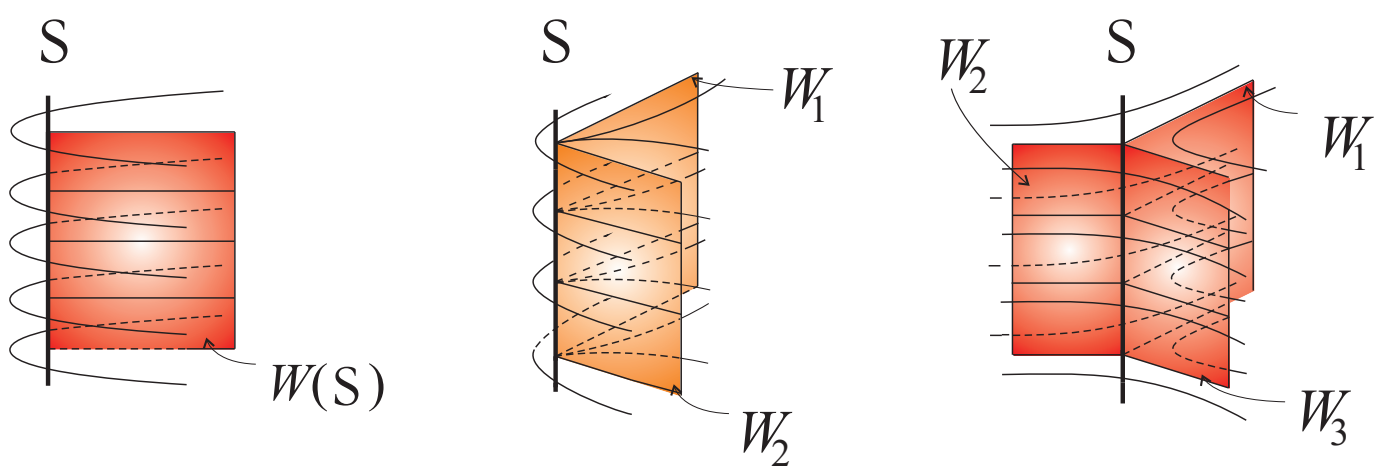

Figura 1.1: Comportamento de $\mathcal{F}_{1}(\alpha)$ numa vizinhança de uma curva parcialmente umbílica Darbouxiana $c(\alpha)$ do tipo $D_{i}$, veja seção 2.2 do capítulo 2. O mesmo acontece para a folheação $\mathcal{F}_{2}(\alpha)$. A folheação $\mathcal{F}_{3}(\alpha)$ é regular. 

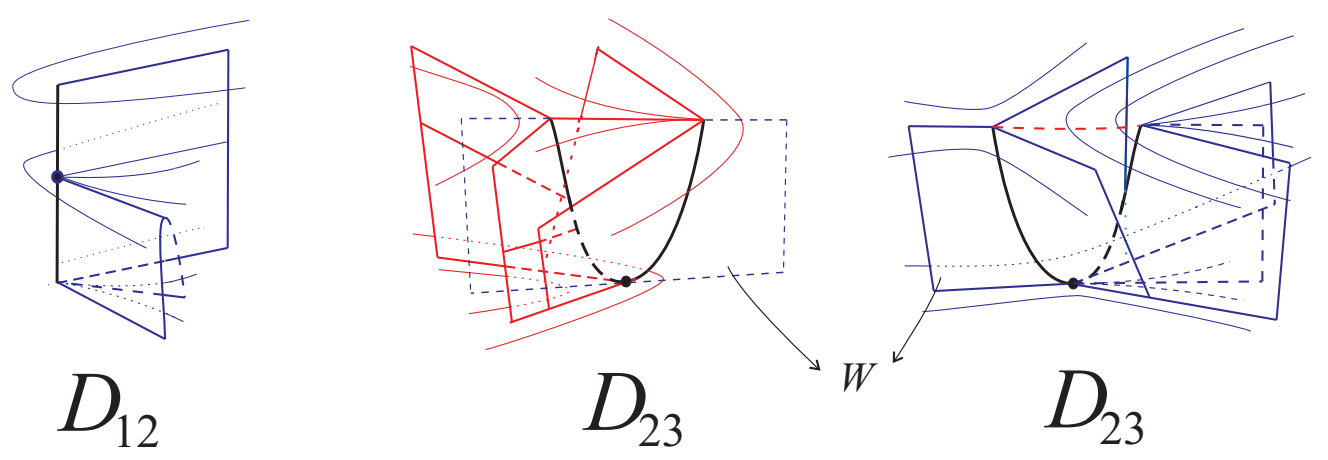

Figura 1.2: Comportamento de $\mathcal{F}_{2}(\alpha)$ numa vizinhança de uma linha parcialmente umbílica contendo um ponto do tipo $D_{12}$ (esquerda), e de $\mathcal{F}_{1}(\alpha)$ e $\mathcal{F}_{2}(\alpha)$ numa vizinhança de uma linha parcialmente umbílica contendo um ponto do tipo $D_{23}$ (centro e direita)

Observação 3 As folhas nas figuras 1.1 e 1.2 são unidimensionais. 


\subsection{Levantamento de Lie-Cartan}

O levantamento que descreveremos, para o estudo de um ponto parcialmente umbílico, consiste basicamente em definir um campo vetorial local, dito de Lie-Cartan, tangente a uma hipersuperfície no fibrado projetivo de $\mathbb{M}^{3}$, que é o levantamento das direções principais tangentes às folheações $\mathcal{F}_{1}(\alpha)$ e $\mathcal{F}_{2}(\alpha)$, e cujas curvas integrais se projetam nas linhas principais da imersão $\alpha$.

Para isto, encontraremos o campo de planos, $q \mapsto \Pi(q)$, ortogonal à direção principal $e_{3}(q)$ (direção regular) correspondente a $k_{3}$, numa vizinhança de um ponto parcialmente umbílico.

Tais planos são definidos por

$$
\Pi(q)=\left\{\left(d u_{1}, d u_{2}, d u_{3}\right) ;\left\langle\left(d u_{1}, d u_{2}, d u_{3}\right), G \cdot\left(e_{3}(q)\right)^{T}\right\rangle=0\right\},
$$

onde $G=\left[g_{i j}\right]_{3 \times 3}$ é a matriz associada à primeira forma fundamental, definida anteriormente.

Resolvendo as duas primeiras equações de (1.3) em $d u_{1}$, $d u_{2}$ com $i=3$, encontramos que $e_{3}(q)=\left(d u_{1}, d u_{2}, d u_{3}\right)$ satisfaz:

$$
\begin{aligned}
& \frac{d u_{1}}{d u_{3}}=\frac{U_{1}\left(u_{1}, u_{2}, u_{3}\right)}{W_{1}\left(u_{1}, u_{2}, u_{3}\right)} \\
& \frac{d u_{2}}{d u_{3}}=\frac{V_{1}\left(u_{1}, u_{2}, u_{3}\right)}{W_{1}\left(u_{1}, u_{2}, u_{3}\right)}
\end{aligned}
$$

onde

$$
\begin{aligned}
U_{1} & =\left(g_{12} g_{23}-g_{22} g_{13}\right) k_{3}^{2}+\left(-g_{12} \lambda_{23}-g_{23} \lambda_{12}+\lambda_{22} g_{13}+g_{22} \lambda_{13}\right) k_{3}+ \\
& +\lambda_{23} \lambda_{12}-\lambda_{22} \lambda_{13} \\
V_{1} & =\left(-g_{11} g_{23}+g_{13} g_{12}\right) k_{3}^{2}+\left(\lambda_{11} g_{23}+g_{11} \lambda_{23}-\lambda_{13} g_{12}-g_{13} \lambda_{12}\right) k_{3}+ \\
& -\lambda_{11} \lambda_{23}+\lambda_{13} \lambda_{12} \\
W_{1} & =\left(g_{11} g_{22}-g_{12}^{2}\right) k_{3}^{2}+\left(-\lambda_{11} g_{22}-g_{11} \lambda_{22}+2 \lambda_{12} g_{12}\right) k_{3}+ \\
& +\lambda_{11} \lambda_{22}-\lambda_{12}^{2}
\end{aligned}
$$

Assim, o plano procurado satisfaz a equação

$$
\left\langle\left(d u_{1}, d u_{2}, d u_{3}\right), G \cdot\left(\frac{U_{1}\left(u_{1}, u_{2}, u_{3}\right)}{W_{1}\left(u_{1}, u_{2}, u_{3}\right)}, \frac{V_{1}\left(u_{1}, u_{2}, u_{3}\right)}{W_{1}\left(u_{1}, u_{2}, u_{3}\right)}, 1\right)^{T}\right\rangle=0,
$$


que escrevemos da forma

$$
\begin{aligned}
d u_{3} & =\mathcal{U}\left(u_{1}, u_{2}, u_{3}\right) d u_{1}+\mathcal{V}\left(u_{1}, u_{2}, u_{3}\right) d u_{2}, \\
\mathcal{U} & =\frac{\left[g_{11} \frac{U_{1}}{W_{1}}+g_{12} \frac{V_{1}}{W_{1}}+g_{13}\right]}{\left[g_{13} \frac{U_{1}}{W_{1}}+g_{23} \frac{V_{1}}{W_{1}}+g_{33}\right]}, \mathcal{V}=\frac{\left[g_{12} \frac{U_{1}}{W_{1}}+g_{22} \frac{V_{1}}{W_{1}}+g_{23}\right]}{\left[g_{13} \frac{U_{1}}{W_{1}}+g_{23} \frac{V_{1}}{W_{1}}+g_{33}\right]} .
\end{aligned}
$$

Disto,

$$
\Pi(q)=\left\{\left(d u_{1}, d u_{2}, d u_{3}\right) \in T_{q} \mathbb{M}^{3} ; d u_{3}=\mathcal{U}\left(u_{1}, u_{2}, u_{3}\right) d u_{1}+\mathcal{V}\left(u_{1}, u_{2}, u_{3}\right) d u_{2}\right\}
$$

Observação 4 Este campo de planos é, em geral, não integrável. De fato, dada a um forma

$$
\begin{aligned}
\omega & =\left[g_{11} \frac{U_{1}}{W_{1}}+g_{12} \frac{V_{1}}{W_{1}}+g_{13}\right] d u_{1}+\left[g_{12} \frac{U_{1}}{W_{1}}+g_{22} \frac{V_{1}}{W_{1}}+g_{23}\right] d u_{2}, \\
& +\left[g_{13} \frac{U_{1}}{W_{1}}+g_{23} \frac{V_{1}}{W_{1}}+g_{33}\right] d u_{3},
\end{aligned},
$$

que define o campo de planos, teremos que, na carta de Monge reduzida $\left(\right.$ com $\left.k_{3}=1\right)$, a condição de integrabilidade de Frobenius $\omega \wedge d \omega=0$ não é válida, numa vizinhança da origem, pois

$$
(\omega \wedge d \omega)\left(u_{1}, u_{2}, u_{3}\right)=F\left(u_{1}, u_{2}, u_{3}\right) d u_{1} \wedge d u_{2} \wedge d u_{3}
$$

onde, considerando apenas os termos de primeira ordem, teremos que

$$
F\left(u_{1}, u_{2}, u_{3}\right)=\frac{\left(a q_{111}-q_{111} b\right)}{k^{2}-2 k+1} u_{1}+\frac{\left(q_{021} b-c q_{111}-q_{201} b\right)}{k^{2}-2 k+1} u_{2}+\text { h.o.t. }
$$

implicando que $\omega \wedge d \omega \neq 0$. Assim, o estudo que desenvolveremos neste trabalho é estritamente tridimensional.

As direções principais $e_{1}(q)$ e $e_{2}(q)$ associadas a $k_{1}(q)$ e $k_{2}(q)$ pertencem a $\Pi(q)$, e são obtidas calculando-se os valores críticos de $k_{n}^{r}(q, \cdot)=\frac{I I_{r}}{I_{r}}(q, \cdot)$ onde $I_{r}(q)$ e $I I_{r}(q)$ são as restrições das formas fundamentais de $\alpha$ ao plano $\Pi(q)$, ou seja,

$$
\begin{aligned}
I_{r}\left(d u_{1}, d u_{2}\right) & =\left.I_{\alpha}\right|_{d u_{3}=\mathcal{U}\left(u_{1}, u_{2}, u_{3}\right) d u_{1}+\mathcal{V}\left(u_{1}, u_{2}, u_{3}\right) d u_{2}} \\
& =E_{r} d u_{1}^{2}+2 F_{r} d u_{1} d u_{2}+G_{r} d u_{2}^{2}, \\
I I_{r}\left(d u_{1}, d u_{2}\right) & =\left.I I_{\alpha}\right|_{d u_{3}=\mathcal{U}\left(u_{1}, u_{2}, u_{3}\right) d u_{1}+\mathcal{V}\left(u_{1}, u_{2}, u_{3}\right) d u_{2}} \\
& =e_{r} d u_{1}^{2}+2 f_{r} d u_{1} d u_{2}+g_{r} d u_{2}^{2},
\end{aligned}
$$


onde

$$
\begin{aligned}
& E_{r}=\frac{\partial^{2} I_{r}}{2 \partial d u_{1}^{2}}(0,0), F_{r}=\frac{\partial^{2} I_{r}}{2 \partial d u_{1} \partial d u_{2}}(0,0), G_{r}=\frac{\partial^{2} I_{r}}{2 \partial d u_{2}^{2}}(0,0), \\
& e_{r}=\frac{\partial^{2} I I_{r}}{2 \partial d u_{1}^{2}}(0,0), f_{r}=\frac{\partial^{2} I I_{r}}{2 \partial d u_{1} \partial d u_{2}}(0,0) \text { e } g_{r}=\frac{\partial^{2} I I_{r}}{2 \partial d u_{2}^{2}}(0,0) .
\end{aligned}
$$

Fazendo $P=\frac{d u_{2}}{d u_{1}}$ em $k_{n}^{r}\left(q ; d u_{1}, d u_{2}\right)$, derivando com relação à $P$ e igualando a zero, teremos que $e_{1}(q)$ e $e_{2}(q)$ são obtidas resolvendo

$$
L_{r}\left(u_{1}, u_{2}, u_{3}\right) P^{2}+M_{r}\left(u_{1}, u_{2}, u_{3}\right) P+N_{r}\left(u_{1}, u_{2}, u_{3}\right)=0,
$$

onde

$$
L_{r}=F_{r} g_{r}-f_{r} G_{r}, \quad M_{r}=E_{r} g_{r}-e_{r} G_{r}, \quad N_{r}=E_{r} f_{r}-e_{r} F_{r}
$$

Tais direções não estão definidas se $k_{1}(q)=k_{2}(q)$, ou seja, nos pontos onde

$$
\left\{\begin{array}{l}
L_{r}\left(u_{1}, u_{2}, u_{3}\right)=0 \\
M_{r}\left(u_{1}, u_{2}, u_{3}\right)=0
\end{array}\right.
$$

Observação 5 1) A função $N_{r}$ é combinação linear das funções $L_{r}$ e $M_{r}$.

2) As expressões para $E_{r}, G_{r}, F_{r}, e_{r}, g_{r}, f_{r}, L_{r}, M_{r}$ e $N_{r}$, na carta de Monge reduzida por homotetia e rotação, são dadas no apêndice: ver (1.25), (1.26), (1.27), (1.28), (1.29), (1.30), (1.31), (1.32) e (1.33).

3) Os pontos parcialmente umbílicos são as soluções de (1.14).

Proposição 1 Se p é um ponto parcialmente umbilico Darbouxiano do tipo $D_{i}(i=$ $1,2,3)$ então p pertence a uma curva regular formada por pontos parcialmente umbílicos do mesmo tipo $D_{i}(i=1,2,3)$.

Demonstração: Suponhamos que $p$ é levado na origem do $\mathbb{R}^{3}$ pela carta $\left(u_{1}, u_{2}, u_{3}\right)$ : $\mathbb{M}^{3} \rightarrow \mathbb{R}^{3}$. Como vimos na seção anterior, os pontos parcialmente umbílicos, numa vizinhança de $p$, são dados por (1.14). Escrevendo $L_{r}$ e $M_{r}$ na carta de Monge reduzida (ver $(1.31)$ e (1.32)), teremos que

$$
\left.\frac{\partial\left(L_{r}, M_{r}\right)}{\partial\left(u_{1}, u_{2}, u_{3}\right)}\right|_{u_{1}=0, u_{2}=0, u_{3}=0}=\left(\begin{array}{ccc}
0 & -b & -q_{111} \\
-a+b & c & -q_{201}+q_{021}
\end{array}\right) .
$$


portanto, se $b(b-a) \neq 0$ então os pontos parcialmente umbílicos, em torno de $p$, formam uma curva regular $c$ de pontos parcialmente umbílicos. Por serem as condições que definem os tipos $D_{1}, D_{2}$ e $D_{3}$ abertas (ver definição 1 ), segue-se que se $p \in c$ é do tipo $D_{i}$ então $q \in c$, suficientemente próximo de $p$, também é do mesmo tipo $D_{i}$.

Observação 6 Por (1.15), vemos também que um ponto parcialmente umbílico semiDarbouxiano pertence a uma curva regular formada por pontos parcialmente umbílicos. Para mais detalhes, ver lemas 3 e 6 do capitulo 2.

Definição 4 Sejam $p \in \mathbb{M}^{3}$ um ponto parcialmente umbílico tal que $k_{1}(p)=k_{2}(p)=$ $k(p) \neq k_{3}(p)=1 \quad e\left(u_{1}, u_{2}, u_{3}\right): \mathbb{M}^{3} \rightarrow \mathbb{R}^{3}$ a carta de Monge reduzida. O ponto $p$ é dito regular se matriz (1.15) tem posto 2 , e é dito crítico se (1.15) tem posto 1.

Definição 5 Suponha $p$ um ponto parcialmente umbilico regular. Se a matriz formada pelas duas primeiras colunas de (1.15) tem determinante diferente de zero diremos que $p$ é bi-regular (transversal), caso contrário, diremos que p é regular-tangente.

A figura 1.3 mostra a curva parcialmente umbílica, composta por pontos parcialmente umbílicos regulares, e os diferentes contatos com o plano umbílico , i. e., plano gerado por $\left\{e_{1}(p), e_{2}(p)\right\}$, onde $p$ é um ponto parcialmente umbílico. 


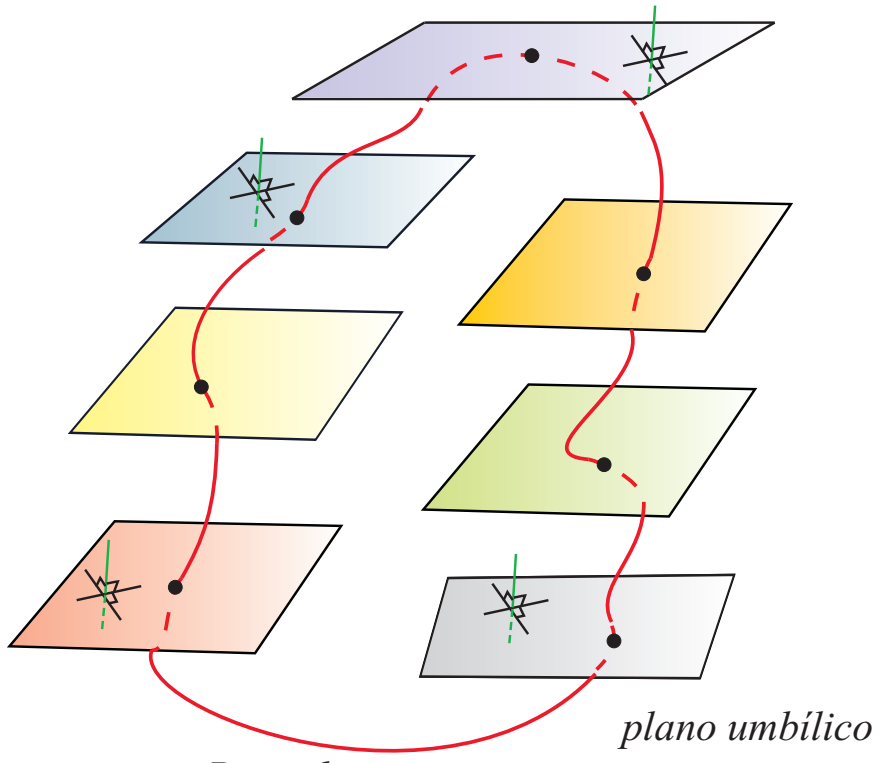

curva Parcialmente umbílica

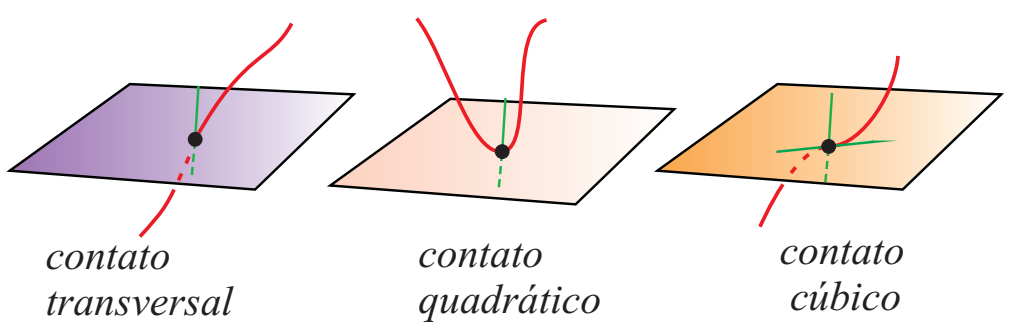

Figura 1.3: Contato da curva parcialmente umbílica com o plano umbílico.

Seja

$$
\mathcal{L}\left(u_{1}, u_{2}, u_{3}, P\right)=L_{r}\left(u_{1}, u_{2}, u_{3}\right) P^{2}+M_{r}\left(u_{1}, u_{2}, u_{3}\right) P+N_{r}\left(u_{1}, u_{2}, u_{3}\right),
$$

a equação

$$
\mathcal{L}\left(u_{1}, u_{2}, u_{3} ; P\right)=0
$$

define uma hipersuperfície no fibrado tangente projetivo $\mathbb{P M}^{3}$ sobre um aberto de $\mathbb{M}^{3}$ se (1.15) tem posto 2. Tal hipersuperfície será chamada hipersuperfície de Lie-Cartan.

Encontraremos um campo

$$
X:\left(u_{1}, u_{2}, u_{3}, P\right) \rightarrow\left\{\begin{array}{l}
\dot{u}_{1}=X_{1}\left(u_{1}, u_{2}, u_{3}, P\right) \\
\dot{u}_{2}=X_{2}\left(u_{1}, u_{2}, u_{3}, P\right) \\
\dot{u}_{3}=X_{3}\left(u_{1}, u_{2}, u_{3}, P\right) \\
\dot{P}=X_{4}\left(u_{1}, u_{2}, u_{3}, P\right)
\end{array}\right.
$$


tangente à hipersuperfície $\mathcal{L}=0$ e tal que a projeção $\Pi\left(u_{1}, u_{2}, u_{3}, P\right)=\left(u_{1}, u_{2}, u_{3}\right)$ das suas curvas integrais sejam as linhas de curvatura principal. Por (1.11) e sendo $P=\frac{d u_{2}}{d u_{1}}$ segue-se que,

$$
\dot{u}_{1}=1, \dot{u}_{2}=P, \dot{u}_{3}=\mathcal{U}+\mathcal{V} P .
$$

Impondo que o campo $X$ seja tangente à hipersuperfície $\mathcal{L}$, ou seja, que ${ }^{2}$

$$
\mathcal{L}_{u_{1}} X_{1}+\mathcal{L}_{u_{2}} X_{2}+\mathcal{L}_{u_{3}} X_{3}+\mathcal{L}_{P} X_{4}=0
$$

obtemos que

$$
\dot{P}=-\frac{\mathcal{L}_{u_{1}}+P \mathcal{L}_{u_{2}}+\mathcal{L}_{u_{3}}(\mathcal{U}+\mathcal{V} P)}{\mathcal{L}_{P}}
$$

Para efeito de simplificação, multiplicamos $\dot{u}_{1}, \dot{u}_{2}, \dot{u}_{3}$ e $\dot{P}$ por $\mathcal{L}_{P}$ obtendo o campo

$$
X:=\left\{\begin{array}{l}
\dot{u}_{1}=\mathcal{L}_{P} \\
\dot{u}_{2}=P \mathcal{L}_{P} \\
\dot{u}_{3}=(\mathcal{U}+\mathcal{V} P) \mathcal{L}_{P} \\
\dot{P}=-\left(\mathcal{L}_{u_{1}}+P \mathcal{L}_{u_{2}}+\mathcal{L}_{u_{3}}(\mathcal{U}+\mathcal{V} P)\right)
\end{array}\right.
$$

Além da carta $\left(u_{1}, u_{2}, u_{3} ; P\right)$, precisamos da carta $\left(u_{1}, u_{2}, u_{3} ; Q\right), Q=\frac{d u_{1}}{d u_{2}}$. Nesta carta, o campo de Lie-Cartan é escrito da forma:

$$
Y:=\left\{\begin{aligned}
\dot{u}_{1} & =Q \frac{\partial \mathcal{G}}{\partial Q} \\
\dot{u}_{2} & =\frac{\partial \mathcal{G}}{\partial Q} \\
\dot{u}_{3} & =(\mathcal{U} Q+\mathcal{V}) \frac{\partial \mathcal{G}}{\partial Q} \\
\dot{Q} & =-\left(Q \mathcal{G}_{u_{1}}+\mathcal{G}_{u_{2}}+\mathcal{G}_{u_{3}}(\mathcal{U} Q+\mathcal{V})\right)
\end{aligned}\right.
$$

onde $\mathcal{G}\left(u_{1}, u_{2}, u_{3}, Q\right)=L_{r}\left(u_{1}, u_{2}, u_{3}\right)+M_{r}\left(u_{1}, u_{2}, u_{3}\right) Q+N_{r}\left(u_{1}, u_{2}, u_{3}\right) Q^{2}$.

Os equilíbrios de $X$ (respectivamente $Y$ ) são as soluções de

$$
\begin{gathered}
\left\{\begin{array}{l}
\mathcal{L}_{P}\left(u_{1}, u_{2}, u_{3}, P\right)=0 \\
\left(\mathcal{L}_{u_{1}}+P \mathcal{L}_{u_{2}}+\mathcal{L}_{u_{3}}(\mathcal{U}+\mathcal{V} P)\right)\left(u_{1}, u_{2}, u_{3}, P\right)=0
\end{array}\right. \\
\left(\begin{array}{l}
\text { respectivamente de }\left\{\begin{array}{l}
\mathcal{G}_{Q}\left(u_{1}, u_{2}, u_{3}, Q\right)=0 \\
\left(Q \mathcal{G}_{u_{1}}+\mathcal{G}_{u_{2}}+\mathcal{G}_{u_{3}}(\mathcal{U} Q+\mathcal{V})\right)\left(u_{1}, u_{2}, u_{3}, Q\right)=0
\end{array}\right) . \\
{ }^{2} \mathcal{L}_{u_{i}}=\frac{\partial \mathcal{L}}{\partial u_{i}}(i=1,2,3), \quad \mathcal{L}_{P}=\frac{\partial \mathcal{L}}{\partial P}
\end{array}\right.
\end{gathered}
$$


A primeira equação do sistema (1.19) se anula no conjunto parcialmente umbílico e a segunda é uma equação cúbica em $P$ (respectivamente $Q$ ), donde vemos que a projeção $\Pi\left(u_{1}, u_{2}, u_{3}, P\right)=\left(u_{1}, u_{2}, u_{3}\right)$ (respectivamente para $(1.20) \Pi\left(u_{1}, u_{2}, u_{3}, Q\right)=$ $\left.\left(u_{1}, u_{2}, u_{3}\right)\right)$ dos equilíbrios de $X$ (respectivamente $Y$ ), na hipersuperfície, são os pontos parcialmente umbílicos. Além disso, por construção, as curvas integrais de $X$ (respectivamente $Y$ ) se projetam nas linhas de curvatura de uma das folheações, $\mathcal{F}_{1}$ ou $\mathcal{F}_{2}$, da imersão.

Nos próximos capítulos, usaremos o levantamento de Lie-Cartan para reduzir o estudo das linhas de curvatura, numa vizinhança de pontos parcialmente umbílicos, ao estudo de curvas integrais de campos vetoriais, numa vizinhança dos seus equilíbrios. 


\subsection{Apêndice: Cálculos até ordem 4}

Nesta seção, usando a carta de Monge reduzida com $h$ escrita até ordem 4 , encontraremos as formas fundamentais de $\alpha$, o campo de planos (definido em (1.9)) e as formas fundamentais restritas a este campo de planos. Além disso, escrevemos as expressões das funções $L_{r}, M_{r}$ e $N_{r}$, que aparecem na definição da hipersuperfície de Lie-Cartan (ver (1.13)).

\subsubsection{Coeficientes das formas fundamentais na carta de Monge reduzida}

Para facilitar a apresentação, reescrevemos (1.4) na forma

$$
\alpha\left(u_{1}, u_{2}, u_{3}\right)=\left(u_{1}, u_{2}, u_{3}, h\left(u_{1}, u_{2}, u_{3}\right)\right)
$$

onde

$$
\begin{aligned}
h\left(u_{1}, u_{2}, u_{3}\right) & =\frac{k}{2}\left(u_{1}^{2}+u_{2}^{2}\right)+\frac{k_{3}}{2} u_{3}^{2}+\frac{1}{6} a u_{1}^{3}+\frac{1}{2} b u_{1} u_{2}^{2}+\frac{1}{6} c u_{2}^{3}+\frac{1}{6} q_{003} u_{3}^{3} \\
& +\frac{1}{2} q_{012} u_{2} u_{3}^{2}+q_{111} u_{1} u_{2} u_{3}+\frac{1}{2} q_{021} u_{2}^{2} u_{3}+\frac{1}{2} q_{102} u_{1} u_{3}^{2} \\
& +\frac{1}{2} q_{201} u_{1}^{2} u_{3}+\frac{1}{24} A u_{1}^{4}+\frac{1}{6} B u_{1}^{3} u_{2}+\frac{1}{4} C u_{1}^{2} u_{2}^{2}+\frac{1}{6} D u_{1} u_{2}^{3} \\
& +\frac{1}{24} E u_{2}^{4}+\frac{1}{24} Q_{004} u_{3}^{4}+\frac{1}{6} Q_{013} u_{2} u_{3}^{3}+\frac{1}{6} Q_{103} u_{1} u_{3}^{3}+\frac{1}{4} Q_{022} u_{2}^{2} u_{3}^{2} \\
& +\frac{1}{4} Q_{202} u_{1}^{2} u_{3}^{2}+\frac{1}{2} Q_{112} u_{1} u_{2} u_{3}^{2}+\frac{1}{6} Q_{031} u_{2}^{3} u_{3}+\frac{1}{6} Q_{301} u_{1}^{3} u_{3} \\
& +\frac{1}{2} Q_{121} u_{1} u_{2}^{2} u_{3}+\frac{1}{2} Q_{211} u_{1}^{2} u_{2} u_{3}+O(5) .
\end{aligned}
$$

Fazendo uma homotetia, podemos supor que $k_{3}(p)=1$. Então:

$$
\begin{aligned}
\frac{\partial h}{\partial u_{1}} & =u_{1} k+\frac{1}{2} a u_{1}^{2}+\frac{1}{2} b u_{2}^{2}+\frac{1}{2} q_{102} u_{3}^{2}+q_{201} u_{1} u_{3}+q_{111} u_{2} u_{3}+\frac{1}{6} Q_{103} u_{3}^{3} \\
& +\frac{1}{2} B u_{1}^{2} u_{2}+\frac{1}{6} D u_{2}^{3}+\frac{1}{2} Q_{202} u_{1} u_{3}^{2}+\frac{1}{2} Q_{301} u_{1}^{2} u_{3}+\frac{1}{2} Q_{112} u_{3}^{2} u_{2} \\
& +Q_{211} u_{3} u_{2} u_{1}+\frac{1}{2} Q_{121} u_{3} u_{2}^{2}+\frac{1}{6} A u_{1}^{3}+\frac{1}{2} C u_{1} u_{2}^{2}+O(4),
\end{aligned}
$$




$$
\begin{aligned}
\frac{\partial h}{\partial u_{2}} & =u_{2} k+\frac{1}{2} c u_{2}^{2}+q_{021} u_{2} u_{3}+b u_{1} u_{2}+q_{111} u_{1} u_{3}+\frac{1}{2} D u_{1} u_{2}^{2}+\frac{1}{2} q_{012} u_{3}^{2} \\
& +\frac{1}{6} B u_{1}^{3}+\frac{1}{2} Q_{112} u_{3}^{2} u_{1}+\frac{1}{6} Q_{211} u_{3} u_{1}^{2}+Q_{121} u_{3} u_{2} u_{1}+\frac{1}{2} Q_{022} u_{2} u_{3}^{2} \\
& +\frac{1}{6} E u_{2}^{3}+\frac{1}{6} Q_{013} u_{3}^{3}+\frac{1}{2} Q_{031} u_{3} u_{2}^{2}+\frac{1}{2} C u_{1}^{2} u_{2}+O(4), \\
\frac{\partial h}{\partial u_{3}}= & u_{3}+\frac{1}{2} q_{021} u_{2}^{2}+\frac{1}{2} q_{201} u_{1}^{2}+q_{102} u_{1} u_{3}+q_{012} u_{3} u_{2}+q_{111} u_{1} u_{2}+\frac{1}{2} q_{003} u_{3}^{2} \\
+ & \frac{1}{6} Q_{301} u_{1}^{3}+Q_{112} u_{3} u_{1} u_{2}+\frac{1}{2} Q_{013} u_{3}^{2} u_{2}+\frac{1}{6} Q_{031} u_{2}^{3}+\frac{1}{2} Q_{202} u_{1}^{2} u_{3} \\
+ & \frac{1}{2} Q_{211} u_{2} u_{1}^{2}+\frac{1}{2} Q_{121} u_{2}^{2} u_{1}+\frac{1}{2} Q_{022} u_{2}^{2} u_{3}+\frac{1}{6} Q_{004} u_{3}^{3}+\frac{1}{2} Q_{103} u_{3}^{2} u_{1} \\
+ & O(4),
\end{aligned}
$$

Disto, expandindo as coordenadas do vetor normal

$$
N=\frac{\frac{\partial h}{\partial u_{1}} \wedge \frac{\partial h}{\partial u_{2}} \wedge \frac{\partial h}{\partial u_{3}}}{\left|\frac{\partial h}{\partial u_{1}} \wedge \frac{\partial h}{\partial u_{2}} \wedge \frac{\partial h}{\partial u_{3}}\right|},
$$

em série de Taylor (numa vizinhança da origem) obtemos que $N=\left(n_{1}, n_{2}, n_{3}, n_{4}\right)$, onde

$$
\begin{aligned}
n_{1} & =-u_{1} k-\frac{1}{2} q_{102} u_{3}^{2}-\frac{1}{2} a u_{1}^{2}-q_{111} u_{2} u_{3}-\frac{1}{2} b u_{2}^{2}-q_{201} u_{1} u_{3}-\frac{1}{2} Q_{112} u_{3}^{2} u_{2} \\
& -Q_{211} u_{1} u_{2} u_{3}-\frac{1}{6} Q_{103} u_{3}^{3}-\frac{1}{2} Q_{121} u_{3} u_{2}^{2}-\frac{1}{6} D u_{2}^{3}+\left(-\frac{1}{2} C+\frac{1}{2} k^{3}\right) u_{1} u_{2}^{2} \\
& +\left(-\frac{1}{2} Q_{202}+\frac{1}{2} k\right) u_{1} u_{3}^{2}-\frac{1}{2} Q_{301} u_{1}^{2} u_{3}+\left(-\frac{1}{6} A+\frac{1}{2} k^{3}\right) u_{1}^{3}-B u_{1}^{2} u_{2} \\
& +O(4), \\
n_{2} & =-u_{2} k-\frac{1}{2} q_{012} u_{3}^{2}-b u_{1} u_{2}-\frac{1}{2} c u_{2}^{2}-q_{111} u_{1} u_{3}-q_{021} u_{2} u_{3}-Q_{121} u_{3} u_{2} u_{1} \\
& -\frac{1}{6} Q_{013} u_{3}^{3}-Q_{031} u_{3} u_{2}^{2}-\frac{1}{2} Q_{112} u_{3}^{2} u_{1}-\frac{1}{2} Q_{211} u_{3} u_{1}^{2}-\frac{1}{2} D u_{1} u_{2}^{2} \\
& +\left(-\frac{1}{2} C+\frac{1}{2} k^{3}\right) u_{1}^{2} u_{2}+\left(-\frac{1}{2} Q_{022}+\frac{1}{2} k\right) u_{2} u_{3}^{2}+\left(-\frac{1}{6} E+\frac{1}{2} k^{3}\right) u_{2}^{3} \\
& -\frac{1}{6} B u_{1}^{3}+O(4),
\end{aligned}
$$




$$
\begin{aligned}
n_{3} & =-u_{3}-\frac{1}{2} q_{201} u_{1}^{2}-\frac{1}{2} q_{003} u_{3}^{2}-q_{102} u_{1} u_{3}-q_{111} u_{1} u_{2}-\frac{1}{2} q_{021} u_{2}^{2}-q_{012} u_{3} u_{2} \\
& -\frac{1}{2} Q_{211} u_{2} u_{1}^{2}-\frac{1}{2} Q_{103} u_{3}^{2} u_{1}+\left(-Q_{202}+\frac{1}{2} k^{2}\right) u_{1}^{2} u_{3}-\frac{1}{2} Q_{013} u_{3}^{2} u_{2} \\
& -\frac{1}{2} Q_{121} u_{2}^{2} u_{1}+\left(-\frac{1}{2} Q_{022}+\frac{1}{2} k^{2}\right) u_{2}^{2} u_{3}-\frac{1}{6} Q_{301} u_{1}^{3}-Q_{112} u_{3} u_{1} u_{2} \\
& -\frac{1}{6} Q_{031} u_{2}^{3}+\left(-\frac{1}{6} Q_{004}+\frac{1}{2}\right) u_{3}^{3}+O(4), \\
n_{4} & =1-\frac{1}{2} u_{2}^{2} k^{2}-\frac{1}{2} u_{1}^{2} k^{2}-\frac{1}{2} u_{3}^{2}-\frac{1}{2} k a u_{1}^{3}+\left(-\frac{1}{2} k-1\right) q_{012} u_{3}^{2} u_{2} \\
& +\left(-\frac{1}{2} k-1\right) q_{102} u_{1} u_{3}^{2}-\frac{1}{2} q_{003} u_{3}^{3}-\frac{3}{2} b k u_{2}^{2} u_{1}+(-2 k-1) q_{111} u_{1} u_{3} u_{2} \\
& -\frac{1}{2} k c u_{2}^{3}+\left(-k-\frac{1}{2}\right) q_{201} u_{3} u_{1}^{2}+\left(-k-\frac{1}{2}\right) q_{021} u_{3} u_{2}^{2}+O(4) .
\end{aligned}
$$

Das equações acima obtemos os coeficientes da primeira e da segunda formas fundamentais denotados, respectivamente, por $g_{i j}$ e $\lambda_{i j}$ :

$$
\begin{aligned}
g_{11} & =\left\langle\alpha_{u_{1}}, \alpha_{u_{1}}\right\rangle=1+k^{2} u_{1}^{2}+k a u_{1}^{3}+b k u_{2}^{2} u_{1}+k q_{102} u_{3}^{2} u_{1}+2 k q_{111} u_{2} u_{3} u_{1} \\
& +2 k q_{201} u_{1}^{2} u_{3}+O(4) \\
g_{12} & =\left\langle\alpha_{u_{1}}, \alpha_{u_{2}}\right\rangle=k^{2} u_{1} u_{2}+k q_{111} u_{3} u_{1}^{2}+\frac{k c}{2} u_{1} u_{2}^{2}+\left(k q_{021}+q_{201} k\right) u_{3} u_{2} u_{1} \\
& +k\left(b+\frac{a}{2}\right) u_{1}^{2} u_{2}+\frac{k q_{012}}{2} u_{3}^{2} u_{1}+\frac{b k}{2} u_{2}^{3}+k q_{111} u_{3} u_{2}^{2}+q_{102} u_{3}^{2} k u_{2}+O(4), \\
g_{13} & =\left\langle\alpha_{u_{1}}, \alpha_{u_{3}}\right\rangle=u_{1} k u_{3}+\frac{k q_{201}}{2} u_{1}^{3}+k q_{111} u_{1}^{2} u_{2}+\left(k q_{102}+\frac{a}{2}\right) u_{3} u_{1}^{2} \\
& +\frac{k q_{021}}{2} u_{2}^{2} u_{1}+k q_{012} u_{3} u_{2} u_{1}+\left(\frac{k q_{003}}{2}+q_{201}\right) u_{3}^{2} u_{1}+\frac{b}{2} u_{3} u_{2}^{2}+q_{111} u_{3}^{2} u_{2} \\
& +\frac{q_{102}}{2} u_{3}^{3}+O(4), \\
g_{22} & =\left\langle\alpha_{u_{2}}, \alpha_{u_{2}}\right\rangle=1+k^{2} u_{2}^{2}+k c u_{2}^{3}+2 k q_{021} u_{2}^{2} u_{3}+k q_{012} u_{3}^{2} u_{2}+2 k b u_{1} u_{2}^{2} \\
& +2 k q_{111} u_{2} u_{3} u_{1}+O(4), \\
g_{23} & =\left\langle\alpha_{u_{2}}, \alpha_{u_{3}}\right\rangle=k u_{2} u_{3}+\frac{1}{2} k q_{201} u_{2} u_{1}^{2}+k q_{111} u_{2}^{2} u_{1}+\left(k q_{102}+b\right) u_{3} u_{2} u_{1} \\
& +q_{111} u_{3}^{2} u_{1}+\frac{1}{2} k q_{021} u_{2}^{3}+\left(k q_{012}+\frac{1}{2} c\right) u_{3} u_{2}^{2}+\left(\frac{1}{2} k q_{003}+q_{021}\right) u_{3}^{2} u_{2} \\
& +\frac{1}{2} q_{012} u_{3}^{3}+O(4),
\end{aligned}
$$




$$
\begin{aligned}
& g_{33}=\left\langle\alpha_{u_{3}}, \alpha_{u_{3}}\right\rangle=1+u_{3}^{2}+q_{021} u_{2}^{2} u_{3}+q_{003} u_{3}^{3}+2 q_{102} u_{1} u_{3}^{2}+q_{201} u_{1}^{2} u_{3} \\
& +2 q_{012} u_{3}^{2} u_{2}+2 q_{111} u_{1} u_{2} u_{3}+O(4), \\
& \lambda_{11}=\left\langle\frac{\partial^{2} \alpha}{\partial u_{1}^{2}}, N\right\rangle=k+a u_{1}+q_{201} u_{3}+\left(-\frac{1}{2} k^{3}+\frac{1}{2} A\right) u_{1}^{2}+B u_{1} u_{2}+Q_{301} u_{1} u_{3} \\
& +\left(-\frac{1}{2} k^{3}+\frac{1}{2} C\right) u_{2}^{2}+Q_{211} u_{3} u_{2}+\left(-\frac{1}{2} k+\frac{1}{2} Q_{202}\right) u_{3}^{2}+O(3) \\
& \lambda_{12}=\left\langle\frac{\partial^{2} \alpha}{\partial u_{1} \partial u_{2}}, N\right\rangle=q_{111} u_{3}+b u_{2}+Q_{121} u_{3} u_{2}+\frac{1}{2} Q_{112} u_{3}^{2}+Q_{211} u_{3} u_{1}+\frac{1}{2} D u_{2}^{2} \\
& +C u_{1} u_{2}+\frac{1}{2} B u_{1}^{2}+O(3) \\
& \lambda_{13}=\left\langle\frac{\partial^{2} \alpha}{\partial u_{1} \partial u_{3}}, N\right\rangle=q_{201} u_{1}+q_{111} u_{2}+q_{102} u_{3}+\frac{1}{2} Q_{103} u_{3}^{2}+Q_{112} u_{3} u_{2}+Q_{211} u_{2} u_{1} \\
& +Q_{202} u_{1} u_{3}+\frac{1}{2} Q_{301} u_{1}^{2}+\frac{1}{2} Q_{121} u_{2}^{2}+O(3), \\
& \lambda_{22}=\left\langle\frac{\partial^{2} \alpha}{\partial u_{2}^{2}}, N\right\rangle=k+c u_{2}+q_{021} u_{3}+b u_{1}+\left(-\frac{1}{2} k^{3}+\frac{1}{2} C\right) u_{1}^{2}+Q_{121} u_{3} u_{1} \\
& +D u_{1} u_{2}+\left(-\frac{1}{2} k^{3}+\frac{1}{2} E\right) u_{2}^{2}+Q_{031} u_{3} u_{2}+\left(-\frac{1}{2} k+\frac{1}{2} Q_{022}\right) u_{3}^{2}+O(3), \\
& \lambda_{23}=\left\langle\frac{\partial^{2} \alpha}{\partial u_{2} \partial u_{3}}, N\right\rangle=q_{012} u_{3}+q_{111} u_{1}+q_{021} u_{2}+\frac{1}{2} Q_{013} u_{3}^{2}+Q_{112} u_{3} u_{1}+Q_{121} u_{2} u_{1} \\
& +\frac{1}{2} Q_{031} u_{2}^{2}+Q_{022} u_{2} u_{3}+\frac{1}{2} Q_{211} u_{1}^{2}+O(3), \\
& \lambda_{33}=\left\langle\frac{\partial^{2} \alpha}{\partial u_{3}^{2}}, N\right\rangle=1+q_{012} u_{2}+q_{102} u_{1}+q_{003} u_{3}+\left(-\frac{1}{2} k^{2}+\frac{1}{2} Q_{202}\right) u_{1}^{2} \\
& +Q_{112} u_{1} u_{2}+Q_{013} u_{3} u_{2}+\left(-\frac{1}{2} k^{2}+\frac{1}{2} Q_{022}\right) u_{2}^{2}+\left(\frac{1}{2} Q_{004}-\frac{1}{2}\right) u_{3}^{2}+O(3),
\end{aligned}
$$




\subsubsection{Expressões das formas fundamentais restritas ao campo de planos ortogonais à direção principal regular}

Usando as expressões para $g_{i j}$ e $\lambda_{i j}$ dadas acima e a equação (1.2), encontraremos o segundo jato ${ }^{3}$ da curvatura principal associada à direção principal regular. Escrevamos

$$
\begin{aligned}
k_{3}\left(u_{1}, u_{2}, u_{3}\right) & =1+a_{100} u_{1}+a_{010} u_{2}+a_{001} u_{3}+\frac{a_{200}}{2} u_{1}^{2}+a_{110} u_{1} u_{2} \\
& +\frac{a_{020}}{2} u_{2}^{2}+a_{101} u_{1} u_{3}+a_{011} u_{2} u_{3}+\frac{a_{002}}{2} u_{3}^{2} .
\end{aligned}
$$

A seguir determinaremos os coeficientes $a_{100}, a_{010}, a_{001}, a_{200}, a_{110}, a_{020}, a_{101}, a_{011}$ e $a_{002}$. Seja $f\left(u_{1}, u_{2}, u_{3}\right)=\operatorname{det}\left(\Lambda\left(u_{1}, u_{2}, u_{3}\right)-k_{3}\left(u_{1}, u_{2}, u_{3}\right) G\left(u_{1}, u_{2}, u_{3}\right)\right)$, onde $\Lambda=\left[\lambda_{i j}\right]_{3 \times 3}$ e $G=\left[g_{i j}\right]_{3 \times 3}$. Então, os coeficientes procurados são obtidos resolvendo o sistema formado pelas seguintes equações:

$$
\begin{aligned}
& \frac{\partial f}{\partial u_{1}}(0,0,0)=0, \frac{\partial f}{\partial u_{2}}(0,0,0)=0, \frac{\partial f}{\partial u_{3}}(0,0,0)=0 \\
& \frac{\partial^{2} f}{\partial u_{1}^{2}}(0,0,0)=0, \frac{\partial^{2} f}{\partial u_{2}^{2}}(0,0,0)=0, \frac{\partial^{2} f}{\partial u_{3}^{2}}(0,0,0)=0 \\
& \frac{\partial^{2} f}{\partial u_{1} \partial u_{2}}(0,0,0)=0, \frac{\partial^{2} f}{\partial u_{1} \partial u_{3}}(0,0,0)=0, \frac{\partial^{2} f}{\partial u_{2} \partial u_{3}}(0,0,0)=0 .
\end{aligned}
$$

Disto,

$$
\begin{aligned}
& a_{100}=q_{102} ; a_{010}=q_{012} ; a_{001}=q_{003} ; \\
& a_{200}=-\frac{k^{3}-k^{2}-k Q_{202}+2 q_{201}^{2}+Q_{202}+2 q_{111}^{2}}{k-1} ; \\
& a_{110}=\frac{k Q_{112}-2 q_{201} q_{111}-Q_{112}-2 q_{111} q_{021}}{k-1} ; \\
& a_{020}=-\frac{k^{3}-k^{2}-k Q_{022}+2 q_{111}{ }^{2}+2 q_{021}{ }^{2}+Q_{022}}{k-1} ; \\
& a_{101}=\frac{k Q_{103}-2 q_{012} q_{111}-2 q_{201} q_{102}-Q_{103}}{k-1} ; \\
& a_{011}=\frac{k Q_{013}-2 q_{102} q_{111}-2 q_{021} q_{012} Q_{013}}{k-1} ; \\
& a_{002}=\frac{k Q_{004}-3 k+3-2 q_{102}{ }^{2}-Q_{004}-2 q_{012}}{k-1} .
\end{aligned}
$$

\footnotetext{
${ }^{3}$ Neste trabalho, só precisaremos do segundo jato da curvatura principal na direção principal regular.
} 
Encontrados $k_{3}$ e os coeficientes das formas fundamentais, obtemos as expressões para $\mathcal{U}\left(u_{1}, u_{2}, u_{3}\right)$ e $\mathcal{V}\left(u_{1}, u_{2}, u_{3}\right)$ em (1.11):

$$
\begin{aligned}
\mathcal{U} & =\frac{1}{2(k-1)^{2}}\left(2 q_{201} u_{1}+2 q_{111} u_{2}+2 q_{102} u_{3}+\left(-2 q_{021} b+2 q_{111} q_{012}+k Q_{121}-\right.\right. \\
& \left.-Q_{121}\right) u_{2}^{2}+\left(2 q_{003} q_{102}-2 q_{201} q_{102}+k Q_{103}-Q_{103}-2 q_{111} q_{012}\right) u_{3}^{2}+\left(2 k^{2}-\right. \\
& \left.-2 q_{201}^{2}-2 Q_{202}-2 k^{3}+2 k Q_{202}+2 q_{102}^{2}-2 q_{111}^{2}-2 a q_{102}+2 q_{201} q_{003}\right) u_{3} u_{1}+ \\
& +\left(-Q_{301}+2 q_{201} q_{102}-2 q_{201} a+Q_{301} k\right) u_{1}^{2}+\left(-2 Q_{112}+2 q_{012} q_{102}-\right. \\
& \left.-2 q_{111} q_{021}+2 q_{003} q_{111}-2 q_{012} b-2 q_{201} q_{111}+2 k Q_{112}\right) u_{3} u_{2}+\left(-2 a q_{111}+\right. \\
& \left.\left.+2 q_{102} q_{111}+2 q_{201} q_{012}-2 q_{111} b-2 Q_{211}+2 Q_{211} k\right) u_{1} u_{2}+O(3)\right) \\
& +\left(-2 q_{111} c-2 q_{201} b+2 k Q_{121}+2 q_{102} q_{021}-2 q_{021} b-2 Q_{121}+2 q_{111} q_{012}\right) u_{1} u_{2}+ \\
& \left.+Q_{211} k\right) u_{1}^{2}+\left(k Q_{013}+2 q_{003} q_{012}-Q_{013}-2 q_{012} q_{021}-2 q_{102} q_{111}\right) u_{3}^{2}+ \\
& +2(k-1)^{2}\left(2 q_{021} u_{2}+2 q_{111} u_{1}+2 q_{012} u_{3}+\left(k Q_{031}-2 q_{111} b-2 q_{021} c-Q_{031}+\right.\right. \\
& \left.+2 q_{012} q_{021}\right) u_{2}^{2}+\left(-2 k^{3}-2 q_{111}^{2}+2 k^{2}-2 q_{012}+2 k Q_{022}-2 Q_{022}-2 q_{021}^{2}-\right. \\
& \left.-2 q_{102} b+2 q_{012}^{2}+2 q_{021} q_{003}\right) u_{3} u_{2}+\left(-2 q_{111} q_{201}+2 q_{003} q_{111}+2 k Q_{112}-\right. \\
& \left.+2 q_{012} q_{102}-2 q_{012} b-2 Q_{112}\right) u_{3} u_{1}+\left(-Q_{211}-2 q_{111} b+2 q_{102} q_{111}+\right. \\
& \\
& +1
\end{aligned}
$$

Daí, as expressões da restrição das formas fundamentais ao campo de planos $q \mapsto \Pi(q)$ 
é dada a seguir:

$$
\begin{gathered}
I_{r}\left(d u_{1}, d u_{2}\right)=\left.I_{\alpha}\right|_{d u_{3}=\mathcal{U} d u_{1}+\mathcal{V} d u_{2}}=E_{r} d u_{1}^{2}+2 F_{r} d u_{1} d u_{2}+G_{r} d u_{2}^{2}, \\
I I_{r}\left(d u_{1}, d u_{2}\right)=\left.I I_{\alpha}\right|_{d u_{3}=\mathcal{U} d u_{1}+\mathcal{V} d u_{2}}=e_{r} d u_{1}^{2}+2 f_{r} d u_{1} d u_{2}+g_{r} d u_{2}^{2}
\end{gathered}
$$

onde, na carta de Monge reduzida,

$$
\begin{aligned}
& E_{r}=1+\left(k^{2}+\frac{q_{201}{ }^{2}}{(k-1)^{2}}\right) u_{1}{ }^{2}+2 \frac{q_{111} q_{201}}{(k-1)^{2}} u_{2} u_{1}+2 \frac{q_{102} q_{201}}{(k-1)^{2}} u_{1} u_{3} \\
& +\frac{q_{111}^{2}}{(k-1)^{2}} u_{2}^{2}+2 \frac{q_{102} q_{111}}{(k-1)^{2}} u_{3} u_{2}+\frac{q_{102}^{2}}{(k-1)^{2}} u_{3}^{2}+O(3) \\
& F_{r}=\frac{2 q_{102} q_{111}+2 q_{201} q_{012}}{(k-1)^{2}} u_{3} u_{1}+\frac{2 q_{102} q_{012}}{(k-1)^{2}} u_{3}^{2}+\frac{2 q_{111} q_{021}}{(k-1)^{2}} u_{2}^{2} \\
& +\left(\frac{2 q_{111}^{2}}{(k-1)^{2}}+k^{2}+\frac{2 q_{201} q_{021}}{(k-1)^{2}}\right) u_{2} u_{1}+\frac{2 q_{102} q_{021}+2_{q 111} q_{012}}{(k-1)^{2}} u_{2} u_{3} \\
& +\frac{2 q_{201} q_{111}}{(k-1)^{2}} u_{1}^{2}+O(3) \\
& G_{r}=1+\frac{q_{012}^{2}}{(k-1)^{2}} u_{3}^{2}+\left(k^{2}+\frac{q_{021}^{2}}{(k-1)^{2}}\right) u_{2}^{2}+\frac{q_{111}^{2}}{(k-1)^{2}} u_{1}^{2} \\
& +\frac{2 q_{021} q_{012}}{(k-1)^{2}} u_{3} u_{2}+\frac{2 q_{111} q_{021}}{(k-1)^{2}} u_{1} u_{2}+\frac{2 q_{012} q_{111}}{(k-1)^{2}} u_{3} u_{1}+O(3) ; \\
& e_{r}=k+a u_{1}+q_{201} u_{3}+\left(\frac{A}{2}+\frac{(2 k-1) q_{201}^{2}}{(k-1)^{2}}-\frac{k^{3}}{2}\right) u_{1}^{2} \\
& +\left(\frac{(2 k-1) q_{111}^{2}}{(k-1)^{2}}+\frac{C}{2}-\frac{k^{3}}{2}\right) u_{2}^{2}+\left(\frac{Q_{202}}{2}+\frac{(2 k-1) q_{102}^{2}}{(k-1)^{2}}-\frac{k}{2}\right) u_{3}^{2} \\
& +\left(B+\frac{(4 k-2) q_{201} q_{111}}{(k-1)^{2}}\right) u_{1} u_{2}+\left(\frac{(4 k-2) q_{102} q_{201}}{(k-1)^{2}}+Q_{301}\right) u_{3} u_{1} \\
& +\left(Q_{211}+\frac{(4 k-2) q_{102} q_{111}}{(k-1)^{2}}\right) u_{3} u_{2}+O(3)
\end{aligned}
$$




$$
\begin{aligned}
& f_{r}=q_{111} u_{3}+b u_{2}+\left(B+\frac{(4 k-2) q_{201} q_{111}}{(k-1)^{2}}\right) u_{1}^{2} \\
& +\left(\frac{(4 k-2) q_{021} q_{111}}{(k-1)^{2}}+D\right) u_{2}^{2}+\left(\frac{(4 k-2) q_{012} q_{102}}{(k-1)^{2}}+Q_{112}\right) u_{3}^{2} \\
& +\left(2 C+\frac{(4 k-2) q_{021} q_{201}}{(k-1)^{2}}+\frac{(4 k-2) q_{111}^{2}}{(k-1)^{2}}\right) u_{1} u_{2} \\
& +\left(2 Q_{211}+\frac{(4 k-2) q_{012} q_{201}}{(k-1)^{2}}+\frac{(4 k-2) q_{102} q_{111}}{(k-1)^{2}}\right) u_{3} u_{1} \\
& +\left(2 Q_{121}+\frac{(4 k-2) q_{012} q_{111}}{(k-1)^{2}}+\frac{(4 k-2) q_{021} q_{102}}{(k-1)^{2}}\right) u_{3} u_{2}+O(3) ; \\
& g_{r}=k+q_{021} u_{3}+b u_{1}+c u_{2}+\left(-\frac{k^{3}}{2}+\frac{C}{2}+\frac{(2 k-1) q_{111}^{2}}{(k-1)^{2}}\right) u_{1}^{2} \\
& +\left(-\frac{k^{3}}{2}+\frac{E}{2}+\frac{(2 k-1) q_{021}{ }^{2}}{(k-1)^{2}}\right) u_{2}^{2}+\left(\frac{Q_{022}}{2}-\frac{k^{3}}{2}+\frac{(2 k-1) q_{012}{ }^{2}}{(k-1)^{2}}\right) u_{3}{ }^{2} \\
& +\left(D+\frac{(-2+4 k) q_{021} q_{111}}{(k-1)^{2}}\right) u_{1} u_{2}+\left(Q_{121}+\frac{(-2+4 k) q_{012} q_{111}}{(k-1)^{2}}\right) u_{3} u_{1} \\
& +\left(Q_{031}+\frac{(-2+4 k) q_{012} q_{021}}{(k-1)^{2}}\right) u_{3} u_{2}+O(3) .
\end{aligned}
$$




\subsubsection{Expressão de $L_{r}, M_{r}$ e $N_{r}$, até segunda ordem, na carta de Monge reduzida.}

Iremos supor $k_{3}=1$.

$$
\begin{aligned}
& L_{r}=-b u_{2}-q_{111} u_{3}-\frac{\left(2 q_{201} q_{111}+B k-B\right)}{2(k-1)} u_{1}^{2}-\left(\frac{\left(2 q_{201} q_{021}+2 q_{111}^{2}\right)}{2(k-1)}+\right. \\
& \left.+C-2 k^{3}\right) u_{1} u_{2}-\left(Q_{211}+\frac{\left(2 q_{102} q_{111}+2 q_{201} q_{012}\right)}{2(k-1)}\right) u_{1} u_{3}-\left(\frac{\left(2 q_{111} q_{021}\right)}{2(k-1)}+\right. \\
& \left.+\frac{D}{2}\right) u_{2}^{2}-\left(Q_{121}+\frac{\left(q_{102} q_{021}+q_{111} q_{012}\right)}{(k-1)}\right) u_{2} u_{3}-\left(\frac{\left(q_{012} q_{102}\right)}{(k-1)}+\right. \\
& \left.+\frac{Q_{112}}{2}\right) u_{3}^{2}+O(3) \\
& M_{r}=(b-a) u_{1}+c u_{2}-\left(q_{201}-q_{021}\right) u_{3}+\left(-\frac{A}{2}+\frac{C}{2}+k^{3}-\frac{q_{201}^{2}-q_{111}^{2}}{k-1}\right) u_{1}^{2}+ \\
& +\left(-B-D-\frac{2 q_{201} q_{111}-2 q_{111} q_{021}}{k-1}\right) u_{1} u_{2}+\left(-\frac{-2 q_{111} q_{012}+2 q_{201} q_{102}}{k-1}+\right. \\
& \left.+Q_{121}-Q_{301}\right) u_{1} u_{3}+\left(\frac{E}{2}-\frac{C}{2}-k^{3}-\frac{q_{111}^{2}-q_{021}^{2}}{k-1}\right) u_{2}^{2}+\left(-Q_{211}+Q_{031}-\right. \\
& \left.-\frac{-2 q_{021} q_{012}+2 q_{102} q_{111}}{k-1}\right) u_{2} u_{3}+\left(\frac{Q_{022}}{2}-\frac{Q_{202}}{2}-\frac{-q_{012}^{2}+q_{102}^{2}}{k-1}\right) u_{3}^{2}+O(3) \\
& N_{r}=b u_{2}+q_{111} u_{3}+\left(\frac{B}{2}+\frac{q_{201} q_{111}}{k-1}\right) u_{1}^{2}+\left(C-k^{3}+\frac{q_{201} q_{021}+q_{111}^{2}}{k-1}\right) u_{1} u_{2}+ \\
& +\left(Q_{211}+\frac{q_{102} q_{111}+q_{201} q_{012}}{k-1}\right) u_{1} u_{3}+\left(\frac{D}{2}+\frac{q_{111} q_{021}}{k-1}\right) v^{2}+\left(Q_{121}+\right. \\
& \left.+\frac{q_{102} q_{021}+q_{111} q_{012}}{k-1}\right) u_{2} u_{3}+\left(\frac{Q_{112}}{2}+\frac{q_{012} q_{102}}{k-1}\right) u_{3}^{2}+O(3)
\end{aligned}
$$




\section{Capítulo 2}

\section{Estrutura das linhas de curvatura numa vizinhança de um ponto parcialmente umbílico Darbouxiano e semi-Darbouxiano}

Neste capítulo, usando o levantamento de Lie-Cartan, daremos uma prova, independente dos resultados de R. Garcia, da estrutura das linhas de curvatura próximas aos pontos parcialmente umbílicos Darbouxianos e semi-Darbouxianos.

Veremos na seção 2.2 que a configuração principal na vizinhança das curvas parcialmente umbílicas dos tipos $D_{1}, D_{2}$ e $D_{3}$ são provenientes da projeção por $\Pi\left(u_{1}, u_{2}, u_{3}, P\right)=\left(u_{1}, u_{2}, u_{3}\right)$ do retrato de fase do campo de Lie-Cartan numa vizinhança de curvas de equilíbrios normalmente hiperbólicas: uma do tipo sela para $D_{1}$, um atrator (ou repulsor) entre duas do tipo sela (para $D_{2}$ ) e três do tipo sela (para $D_{3}$ ). Veja figuras 2.6, 2.7 e 2.8. Quanto aos pontos $D_{23}$ e $D_{12}$, no campo de Lie-Cartan, além dos conjuntos normalmente hiperbólicos aparecem conjuntos do tipo sela-nó, veja as figuras 2.14 e 2.21 .

\section{$2.1 \quad$ Preliminares}

Nesta seção definiremos separatriz parcialmente umbílica e setores parabólicos e hiperbólicos associados às linhas de curvatura em uma variedade de dimensão 3.

Sejam $\mathbb{M}^{3}$ uma variedade compacta de dimensão 3 , orientada e de classe $C^{k}$, 
$k \geq 4$ e $c$ uma curva parcialmente umbílica em $\mathbb{M}^{3}$.

Definição 6 (Superfície Separatriz Parcialmente Umbílica de $c$ ) Um subconjunto $S_{c}$ de $\mathbb{M}^{3}$ é dito Superfície Separatriz Parcialmente Umbilica de c se as seguintes condições forem satisfeitas:

- $S_{c}$ é uma subvariedade de $\mathbb{M}^{3}$, conexa e de dimensão 2;

- $S_{c}$ não possui pontos parcialmente umbílicos;

- $S_{c}$ é invariante pelas linhas de curvatura de uma das folheações;

- c está contida no fecho de $S_{c}$.

Analogamente, definimos separatriz parcialmente umbílica de um ponto parcialmente umbílico $p$ :

Definição 7 (Separatriz Parcialmente Umbílica de $p$ ) Um subconjunto $S_{p}$ de $\mathbb{M}^{3}$ é dito Separatriz Parcialmente Umbílica de p se as seguintes condições forem satisfeitas:

- $S_{p}$ é uma subvariedade de $\mathbb{M}^{3}$, conexa e de dimensão 1 ;

- $S_{p}$ não possui pontos parcialmente umbílicos;

- $S_{p}$ é invariante pelas linhas de curvatura;

- $p$ está contido no fecho de $S_{p}$.

Definiremos a seguir o que são setores parabólicos e hiperbólicos, referentes às linhas de curvatura em $\mathbb{M}^{3}$.

Sejam $c$ uma curva parcialmente umbílica Darbouxiana, $p \in c$ e $V_{p}$ uma vizinhança tubular de $c$, suficientemente pequena e contendo $p$. Neste trabalho, o conjunto das superfícies separatrizes parcialmente umbílicas, relativas a uma mesma folheação principal, particionam $V_{p}$ em um número finito de regiões abertas que denominaremos setores.

Seja $S$ um setor. Dado $q \in S$ denotaremos por $\gamma_{q}$ a linha principal passando por $q$ relativa à mesma folheação principal que define o setor $S$.

- Dizemos que $S$ é um setor parabólico se, para todo $q \in S, \overline{V_{p} \cap \gamma_{q}} \cap c \neq \emptyset \mathrm{e}$ $\partial V_{p} \cap \gamma_{q} \neq \emptyset$. Topologicamente, um setor parabólico é como na figura 2.1. 
- Dizemos que $S$ é um setor hiperbólico se, para todo $q \in S, \overline{V_{p} \cap \gamma_{q}} \cap c=\emptyset$, $\partial S \cap \gamma_{q}=\partial V_{p} \cap \gamma_{q}$ e a fronteira de $V_{p}$ intersecta $\gamma_{q}$ em pelo menos 2 pontos. Topologicamente, um setor hiperbólico é como na figura 2.2.

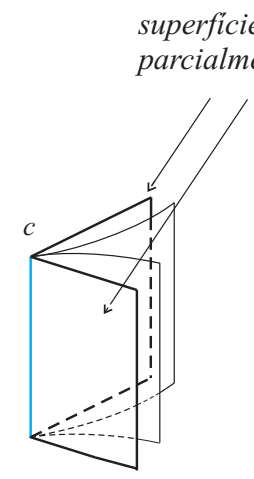

Figura 2.1: Setor Parabólico superficies separatrizes parcialmente umbílicas

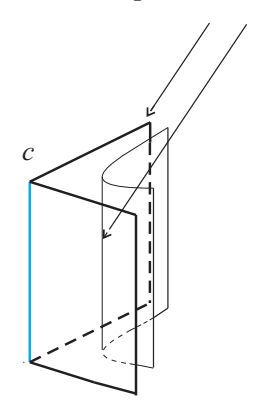

Figura 2.2: Setor Hiperbólico 


\subsection{Pontos Parcialmente Umbílicos Darbouxianos}

Pela proposição 1 do capítulo 1, os pontos parcialmente umbílicos Darbouxianos aparecem em curvas regulares, chamadas curvas parcialmente umbílicas Darbouxianas. A estrutura das linhas de curvatura numa vizinhança de tais curvas é descrita no seguinte teorema:

Teorema 1 (R. Garcia, [6], [7]) Suponhamos $\alpha \in \mathcal{I}^{k}\left(\mathbb{M}^{3}, \mathbb{R}^{4}\right), k \geq 4$. Denotemos por c uma curva parcialmente umbílica Darbouxiana. Então,

(i) Se c é do tipo $D_{1}$, existe uma única superfície separatriz parcialmente umbílica, $W_{c}$, de classe $C^{k-2}$, fibrada sobre c e cujas fibras são folhas de $\mathcal{F}_{1}(\alpha)$. Além disso, existe uma vizinhança tubular $V_{c}$ de c tal que o conjunto $V_{c} \backslash W_{c}$ é um setor hiperbólico de $\mathcal{F}_{1}(\alpha)$.

(ii) Se c é do tipo $D_{2}$, então existem duas superfícies separatrizes parcialmente umbílicas, $W_{1}$ e $W_{2}$, e exatamente um setor parabólico e um setor hiperbólico de $\mathcal{F}_{1}(\alpha)$.

(iii) Se c é do tipo $D_{3}$, então existem três superfícies separatrizes parcialmente umbílicas, $W_{1}, W_{2}$ e $W_{3}$, e exatamente três setores hiperbólicos de $\mathcal{F}_{1}(\alpha)$.

(iv) O mesmo acontece para a folheação $\mathcal{F}_{2}(\alpha)$ que é ortogonal a $\mathcal{F}_{1}(\alpha)$ e singular na curva c. Além disto, as superfícies separatrizes parcialmente umbílicas associadas à $\mathcal{F}_{2}(\alpha)$, no caso $D_{i}$, são tangentes às superfícies separatrizes parcialmente umbilicas associadas à $\mathcal{F}_{1}(\alpha)$ ao longo de c. Veja as figuras 2.3, 2.4 e 2.5. 


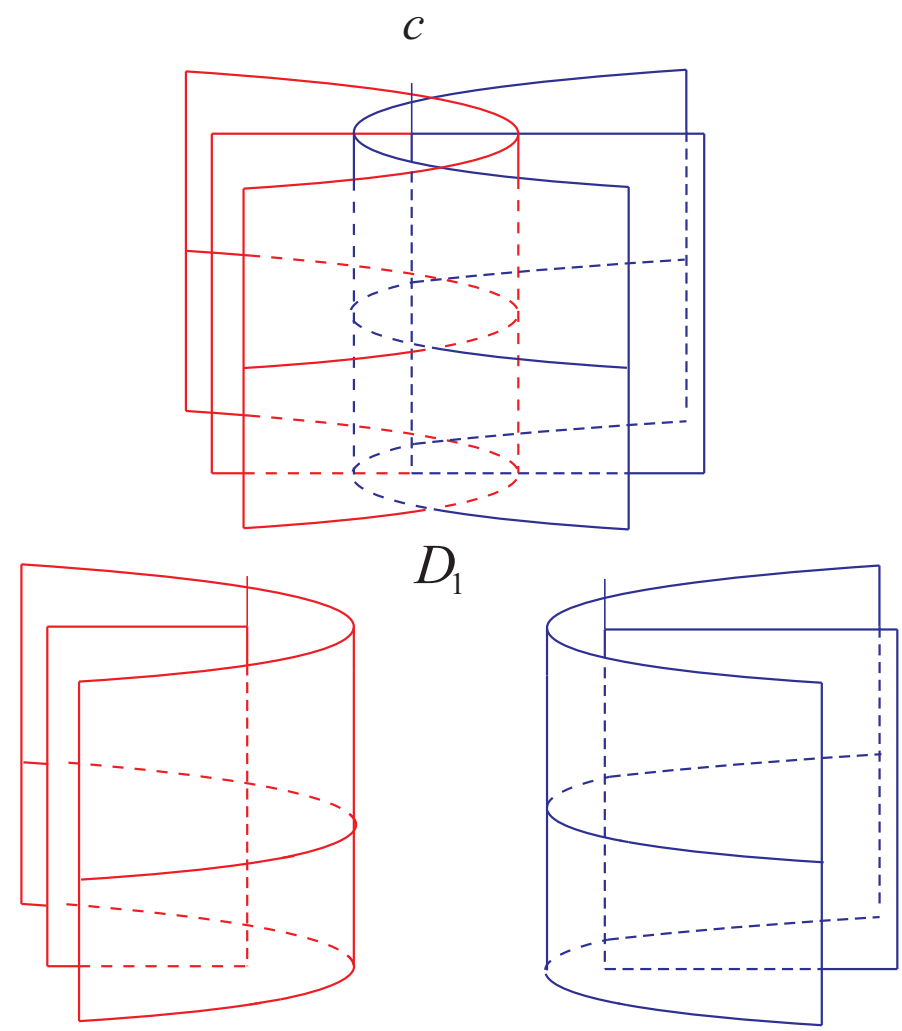

Figura 2.3: Comportamento de $\mathcal{F}_{1}(\alpha)$ e $\mathcal{F}_{2}(\alpha)$ numa vizinhança de uma curva parcialmente umbílica Darbouxiana $c$ do tipo $D_{1}$. As folheações são unidimensionais. 

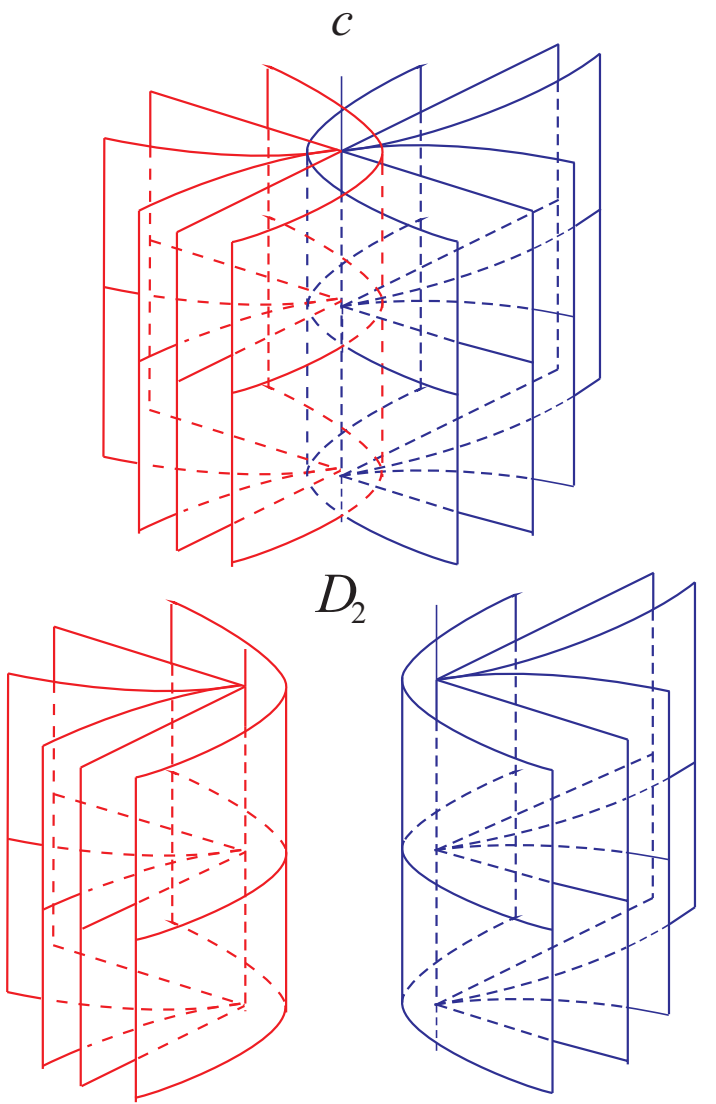

Figura 2.4: Comportamento de $\mathcal{F}_{1}(\alpha)$ e $\mathcal{F}_{2}(\alpha)$ numa vizinhança de uma curva parcialmente umbílica Darbouxiana $c$ do tipo $D_{2}$. As folheações são unidimensionais. 


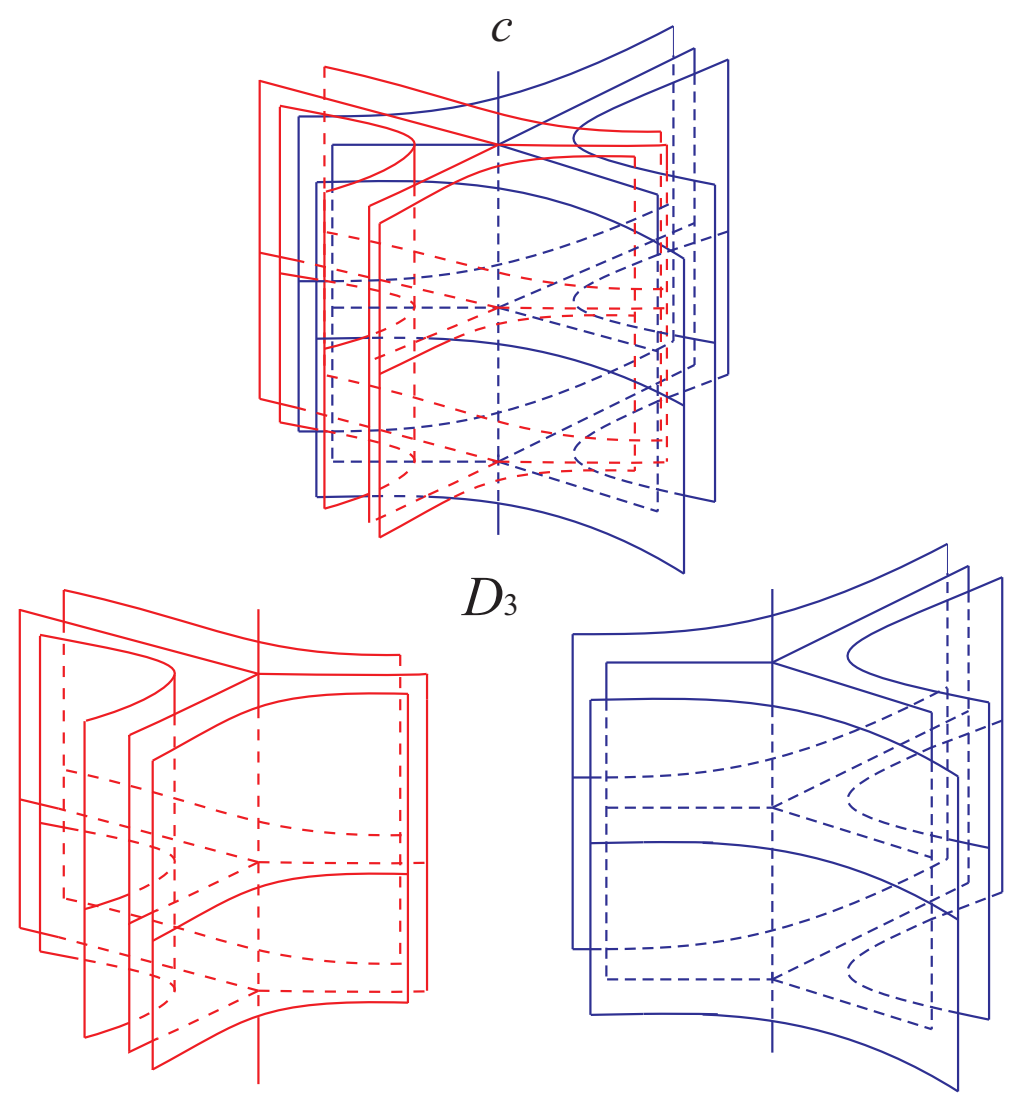

Figura 2.5: Comportamento de $\mathcal{F}_{1}(\alpha)$ e $\mathcal{F}_{2}(\alpha)$ numa vizinhança de uma curva parcialmente umbílica Darbouxiana $c$ do tipo $D_{3}$. As folheações são unidimensionais. 
O objetivo desta seção é demonstrar o teorema 1 usando o levantamento de LieCartan.

Seja $c$ uma curva parcialmente umbílica Darbouxiana do tipo $D_{i},(i=1,2,3)$. No espaço das variáveis $u_{1}, u_{2}$ e $u_{3}$, segue-se de (1.15) que podemos escrever $c$ da forma

$$
u_{1}=c_{1}\left(u_{3}\right), \quad u_{2}=c_{2}\left(u_{3}\right)
$$

Além disso, usando a carta de Monge reduzida com $k_{3}=1$, vemos que

$$
c^{\prime}(0)=\left[\frac{\left(-c q_{111}+q_{021} b-q_{201} b\right)}{b(-b+a)},-\frac{q_{111}}{b}, 1\right] .
$$

Como consequência, teremos que $c$ é transversal ao plano umbílico, i. e., ao plano gerado por $e_{1}(p)$ e $e_{2}(p)$, onde $p=\alpha(0)$ (veja a equação (1.4) no capítulo 1 ).

Para demonstrar o teorema 1, usando o levantamento de Lie-Cartan, devemos analisar o campo de Lie-Cartan, $X$, restrito à hipersuperfície de Lie-Cartan, $\mathcal{L}\left(u_{1}, u_{2}, u_{3}, P\right)=$ 0 , onde

$$
\begin{gathered}
\mathcal{L}\left(u_{1}, u_{2}, u_{3}, P\right)=L_{r}\left(u_{1}, u_{2}, u_{3}\right) P^{2}+M_{r}\left(u_{1}, u_{2}, u_{3}\right) P+N_{r}\left(u_{1}, u_{2}, u_{3}\right), \\
X:=\left\{\begin{array}{l}
\dot{u}_{1}=\mathcal{L}_{P} \\
\dot{u}_{2}=P \mathcal{L}_{P} \\
\dot{u}_{3}=(\mathcal{U}+\mathcal{V} P) \mathcal{L}_{P} \\
\dot{P}=-\left(\mathcal{L}_{u_{1}}+P \mathcal{L}_{u_{2}}+\mathcal{L}_{u_{3}}(\mathcal{U}+\mathcal{V} P)\right)
\end{array}\right.
\end{gathered}
$$

e, como visto em (1.11), as funções $\mathcal{U}$ e $\mathcal{V}$ definem o campo de planos ortogonal à direção principal regular.

Vimos em (1.23), (1.24), (1.31), (1.32) e (1.33) (apêndice do capítulo 1) que, na carta de Monge reduzida com $k_{3}=1$, as funções $\mathcal{U}, \mathcal{V}, L_{r}, M_{r}$ e $N_{r}$ são dadas por 


$$
\begin{aligned}
\mathcal{U} & =\frac{1}{2(k-1)^{2}}\left(2 q_{201} u_{1}+2 q_{111} u_{2}+2 q_{102} u_{3}+\left(-2 q_{021} b+2 q_{111} q_{012}+k Q_{121}-\right.\right. \\
& \left.-Q_{121}\right) u_{2}^{2}+\left(2 q_{003} q_{102}-2 q_{201} q_{102}+k Q_{103}-Q_{103}-2 q_{111} q_{012}\right) u_{3}^{2}+\left(2 k^{2}-\right. \\
& \left.-2 q_{201}^{2}-2 Q_{202}-2 k^{3}+2 k Q_{202}+2 q_{102}^{2}-2 q_{111}^{2}-2 a q_{102}+2 q_{201} q_{003}\right) u_{3} u_{1}+ \\
& +\left(-Q_{301}+2 q_{201} q_{102}-2 q_{201} a+Q_{301} k\right) u_{1}^{2}+\left(-2 Q_{112}+2 q_{012} q_{102}-\right. \\
& \left.-2 q_{111} q_{021}+2 q_{003} q_{111}-2 q_{012} b-2 q_{201} q_{111}+2 k Q_{112}\right) u_{3} u_{2}+\left(-2 a q_{111}+\right. \\
& \left.\left.+2 q_{102} q_{111}+2 q_{201} q_{012}-2 q_{111} b-2 Q_{211}+2 Q_{211} k\right) u_{1} u_{2}+O(3)\right) \\
& + \\
& +\left(-2 q_{111} c-2 q_{201} b+2 k Q_{121}+2 q_{102} q_{021}-2 q_{021} b-2 Q_{121}+2 q_{111} q_{012}\right) u_{1} u_{2}+ \\
& \left.+2 q_{012} q_{021}\right) u_{2}^{2}+\left(-2 k^{3}-2 q_{111}^{2}+2 k^{2}-2 q_{012}+2 k Q_{022}-2 Q_{022}-2 q_{021}^{2}-\right. \\
& \left.+2 q_{102} b+2 q_{012}^{2}+2 q_{021} q_{003}\right) u_{3} u_{2}+\left(-2 q_{111} q_{201}+2 q_{003} q_{111}+2 k Q_{112}-\right. \\
& -2 q_{111} q_{021}+2 u_{12}^{2}+\left(k Q_{013}+2 q_{102}-2 q_{012} b-2 Q_{112}\right) u_{3} u_{1}+\left(-Q_{211}-2 q_{111} b+2 q_{102} q_{111}+\right. \\
& \left.+Q_{013}-2 q_{012} q_{021}-2 q_{102} q_{111}\right) u_{3}^{2}+\left(k Q_{031}-2 q_{111} b-2 q_{021} c-Q_{031}+\right.
\end{aligned}
$$




$$
\begin{aligned}
& L_{r}=-b u_{2}-q_{111} u_{3}-\frac{\left(2 q_{201} q_{111}+B k-B\right)}{2(k-1)} u_{1}^{2}-\left(\frac{\left(2 q_{201} q_{021}+2 q_{111}^{2}\right)}{2(k-1)}+\right. \\
& \left.+C-2 k^{3}+\right) u_{1} u_{2}-\left(Q_{211}+\frac{\left(2 q_{102} q_{111}+2 q_{201} q_{012}\right)}{2(k-1)}\right) u_{1} u_{3}-\left(\frac{\left(2 q_{111} q_{021}\right)}{2(k-1)}+\right. \\
& \left.+\frac{D}{2}\right) u_{2}^{2}-\left(Q_{121}+\frac{\left(q_{102} q_{021}+q_{111} q_{012}\right)}{(k-1)}\right) u_{2} u_{3}-\left(\frac{\left(q_{012} q_{102}\right)}{(k-1)}+\right. \\
& \left.+\frac{Q_{112}}{2}\right) u_{3}^{2}+O(3) \\
& M_{r}=(b-a) u_{1}+c u_{2}-\left(q_{201}-q_{021}\right) u_{3}+\left(-\frac{A}{2}+\frac{C}{2}+k^{3}-\frac{q_{201}^{2}-q_{111}^{2}}{k-1}\right) u_{1}^{2}+ \\
& +\left(-B-D-\frac{2 q_{201} q_{111}-2 q_{111} q_{021}}{k-1}\right) u_{1} u_{2}+\left(-\frac{-2 q_{111} q_{012}+2 q_{201} q_{102}}{k-1}+\right. \\
& \left.+Q_{121}-Q_{301}\right) u_{1} u_{3}+\left(\frac{E}{2}-\frac{C}{2}-k^{3}-\frac{q_{111}^{2}-q_{021}^{2}}{k-1}\right) u_{2}^{2}+\left(-Q_{211}+Q_{031}-\right. \\
& \left.-\frac{-2 q_{021} q_{012}+2 q_{102} q_{111}}{k-1}\right) u_{2} u_{3}+\left(\frac{Q_{022}}{2}-\frac{Q_{202}}{2}-\frac{-q_{012}^{2}+q_{102}^{2}}{k-1}\right) u_{3}^{2}+O(3), \\
& N_{r}=b u_{2}+q_{111} u_{3}+\left(\frac{B}{2}+\frac{q_{201} q_{111}}{k-1}\right) u_{1}^{2}+\left(C-k^{3}+\frac{q_{201} q_{021}+q_{111}^{2}}{k-1}\right) u_{1} u_{2}+ \\
& +\left(Q_{211}+\frac{q_{102} q_{111}+q_{201} q_{012}}{k-1}\right) u_{1} u_{3}+\left(\frac{D}{2}+\frac{q_{111} q_{021}}{k-1}\right) v^{2}+\left(Q_{121}+\right. \\
& \left.+\frac{q_{102} q_{021}+q_{111} q_{012}}{k-1}\right) u_{2} u_{3}+\left(\frac{Q_{112}}{2}+\frac{q_{012} q_{102}}{k-1}\right) u_{3}^{2}+O(3) .
\end{aligned}
$$

Disto, denotando as coordenadas de $X$ por $X_{1}, X_{2}, X_{3}, X_{4}$ teremos que

$$
\begin{aligned}
& X_{1}=\left(-2 b u_{2}-2 q_{111} u_{3}\right) P+(-a+b) u_{1}+c u_{2}+\left(-q_{201}+q_{021}\right) u_{3}+O(2) \\
& X_{2}=P X_{1} \\
& X_{3}=\left(\left(\frac{q_{111} u_{1}+q_{021} u_{2}+q_{012} u_{3}}{(k-1)^{2}}+O(2)\right) P+\frac{q_{201} u_{1}+q_{111} u_{2}+q_{102} u_{3}}{(k-1)^{2}}+O(2)\right) X_{1} \\
& X_{4}=A_{3}\left(u_{1}, u_{2}, u_{3}\right) P^{3}+A_{2}\left(u_{1}, u_{2}, u_{3}\right) P^{2}+A_{1}\left(u_{1}, u_{2}, u_{3}\right) P+A_{0}\left(u_{1}, u_{2}, u_{3}\right)
\end{aligned}
$$


onde

$$
\begin{aligned}
& A_{3}\left(u_{1}, u_{2}, u_{3}\right)=b+\left(C-k^{3}+\frac{q_{111}^{2}+q_{201} q_{021}}{k-1}\right) u_{1}+\left(D+3 \frac{q_{111} q_{021}}{k-1}\right) u_{2}+ \\
& +\left(Q_{121}+\frac{2 q_{111} q_{012}+q_{102} q_{021}}{k-1}\right) u_{3}+O(2) \\
& A_{2}\left(u_{1}, u_{2}, u_{3}\right)=c+\left(-D+2 B+\frac{6 q_{111} q_{201}-3 q_{111} q_{021}}{k-1}\right) u_{1}+ \\
& +\left(-E+k^{3}+2 C+\frac{4 q_{111}^{2}-3 q_{021}^{2}+2 q_{201} q_{021}}{k-1}\right) u_{2}+ \\
& +\left(-Q_{031}+2 \frac{Q_{211}\left(2 q_{201} q_{012}+4 q_{102} q_{111}-3 q_{012} q_{021}\right)}{k-1}\right) u_{3}+O(2) \\
& A_{1}\left(u_{1}, u_{2}, u_{3}\right)=a-2 b+\left(-2 C+A-k^{3}+\frac{-2 q_{201} q_{021}-4 q_{111}^{2}+3 q_{201}^{2}}{k-1}\right) u_{1}+ \\
& +\left(-2 D+B+\frac{3 q_{111} q_{201}-6 q_{111} q_{021}}{k-1}\right) u_{2}+ \\
& +\left(-2 Q_{121}+Q_{301}+\frac{3 q_{102} q_{201}-2 q_{102} q_{021}-4 q_{111} q_{012}}{k-1}\right) u_{3}+O(2) \\
& A_{0}\left(u_{1}, u_{2}, u_{3}\right)=\left(-B-3 \frac{q_{111} q_{201}}{k-1}\right) u_{1}+\left(-C+k^{3}-\frac{2 q_{111}^{2}+q_{201} q_{021}}{k-1}\right) u_{2}+ \\
& +\left(-Q_{211}-\frac{q_{201} q_{012}+2 q_{102} q_{111}}{k-1}\right) u_{3}+O(2)
\end{aligned}
$$

Os equilíbrios de $X$ serão encontrados no lema 1, e a estrutura topológica das curvas integrais de $X$, numa vizinhança dos seus equilíbrios, será dada no lema 2. No final desta seção demonstraremos o teorema 1 usando ambos os lemas.

Lema 1 Sejam X o campo de Lie-Cartan e c uma curva parcialmente umbilica Darbouxiana.

i) Se c é do tipo $D_{1}$ então existe uma única curva de equilíbrios para $X$ que se projeta em $c$.

ii) Se c é do tipo $D_{2}$ ou $D_{3}$ então existem exatamente três curvas de equilíbrios de $X$ que se projetam em $c$.

Demonstração: Seja $\left(u_{1}, u_{2}, u_{3}\right): \mathbb{M}^{3} \rightarrow \mathbb{R}^{3}$ a carta de Monge reduzida com a restrição $k_{3}=1$. De (2.2), vemos que os equilíbrios de $X$ são dados por

$$
\left\{\begin{array}{l}
\mathcal{L}_{P}\left(u_{1}, u_{2}, u_{3}, P\right)=0 \\
\left(\mathcal{L}_{u_{1}}+P \mathcal{L}_{u_{2}}+\mathcal{L}_{u_{3}}(\mathcal{U}+\mathcal{V} P)\right)\left(u_{1}, u_{2}, u_{3}, P\right)=0
\end{array}\right.
$$


Como

$$
\mathcal{L}_{P}\left(u_{1}, u_{2}, u_{3}, P\right)=2 P L_{r}\left(u_{1}, u_{2}, u_{3}\right)+M_{r}\left(u_{1}, u_{2}, u_{3}\right),
$$

segue-se que os pontos parcialmente umbílicos, que neste caso formam uma curva regular $c$, anulam a primeira equação de (2.4). Vimos em (2.1) que podemos escrever as coordenadas de $c$ da forma

$$
u_{1}=c_{1}\left(u_{3}\right), \quad u_{2}=c_{2}\left(u_{3}\right)
$$

e que, além disso,

$$
\begin{aligned}
& c_{1}\left(u_{3}\right)=\frac{\left(-c q_{111}+q_{021} b-q_{201} b\right)}{b(-b+a)} u_{3}+O(2) \\
& c_{2}\left(u_{3}\right)=-\frac{q_{111}}{b} u_{3}+O(2)
\end{aligned}
$$

Substituindo (2.5) e (2.6) na segunda equação de (2.4) e usando (2.3), obtemos a seguinte equação cúbica em $P$ :

$$
(b+O(1)) P^{3}+(-c+O(1)) P^{2}+(a-2 b+O(1)) P+O(1)=0,
$$

onde $O(1)$ representa os termos de ordem maior ou igual a 1 na variável $u_{3}$. O discriminante $^{1}, D\left(u_{3}\right)$, de $(2.7)$ é dado por

$$
D\left(u_{3}\right)=-\left(\frac{c^{2}}{4 b^{2}}-\frac{a}{b}+2\right)\left(\frac{2 a}{b}-4\right)^{2}+O(1),
$$

donde vemos que, para $u_{3}$ suficientemente pequeno (veja definição 1 no capítulo 1 ):

Condição $\left.D_{1}\right) \Rightarrow D\left(u_{3}\right)>0 \Rightarrow$ Existe uma única solução, $P_{1}\left(u_{3}\right)$, de $(2.7)$,

Condições $D_{2}$ e $\left.D_{3}\right) \Rightarrow D\left(u_{3}\right)<0 \Rightarrow$ Existem exatamente 3 soluções de (2.7), que denotaremos por $P_{1}\left(u_{3}\right), P_{2}\left(u_{3}\right)$ e $P_{3}\left(u_{3}\right)$.

\footnotetext{
${ }^{1}$ Lembre que o discriminante de uma equação cúbica $p(x)=x^{3}+\alpha x^{2}+\beta x+\gamma$ é dado por

$$
D=\frac{1}{4}\left(\gamma+\frac{2 \alpha^{3}-9 \alpha \beta}{27}\right)^{2}+\frac{1}{27}\left(\beta-\frac{\alpha^{2}}{3}\right)^{3},
$$
}

com as seguintes propriedades:

$$
\left\{\begin{array}{l}
D=0 \Rightarrow \text { três raizes reais sendo duas iguais } \\
D>0 \Rightarrow \text { uma raiz real e duas complexas conjugadas } \\
D<0 \Rightarrow \text { três raizes reais distintas }
\end{array} .\right.
$$


Como, para $u_{3}=0$, a equação (2.7) tem as seguintes soluções:

$$
\begin{aligned}
& P_{1}(0)=0 \\
& P_{2}(0)=\frac{c}{2 b}+\sqrt{\frac{c^{2}}{4 b^{2}}-\frac{a}{b}+2} \\
& P_{3}(0)=\frac{c}{2 b}-\sqrt{\frac{c^{2}}{4 b^{2}}-\frac{a}{b}+2}
\end{aligned}
$$

concluímos que:

- se $c$ é do tipo $D_{1}$ então existe uma única curva de equilíbrios para $X$ que se projeta em $c$, além disso, tal curva intersecta o eixo $P$ em $P_{1}(0)$,

- se $c$ é do tipo $D_{2}$ ou $D_{3}$ então existem exatamente 3 curvas de equilíbrios de $X$ que se projetam em $c$, tais curvas intersectam o eixo $P$ em $P_{1}(0), P_{2}(0)$ e $P_{3}(0)$.

Para cada $i=1,2,3$, denotaremos a curva de equilíbrios de $X$ intersectando o eixo $P$ em $P=P_{i}(0)$ por $\beta_{i}\left(u_{3}\right)$.

Lema 2 Seja $X_{\mathcal{L}}$ o campo de Lie-Cartan restrito à hipersuperfície de Lie-Cartan. Então:

Condição $\left.D_{1}\right) \Rightarrow \beta_{1}$ é normalmente hiperbólica do tipo sela para $X_{\mathcal{L}}$,

Condição $\left.D_{2}\right) \Rightarrow$ Para $i=1,2,3, \beta_{i}$ é normalmente hiperbólica para $X_{\mathcal{L}}$ satisfazendo:

uma do tipo atratora (ou repulsora) entre duas do tipo sela,

Condição $\left.D_{3}\right) \Rightarrow \beta_{i}$ é normalmente hiperbólica do tipo sela para $X_{\mathcal{L}}, \quad i=1,2,3$.

Demonstração: Seja $X=\left(X_{1}, X_{2}, X_{3}, X_{4}\right)$ o campo de Lie-Cartan dado em (2.2). Analisaremos a linearização de $X$ ao longo das curvas de equilíbrios

$$
\beta_{i}\left(u_{3}\right)=\left(c_{1}\left(u_{3}\right), c_{2}\left(u_{3}\right), u_{3}, P_{i}\left(u_{3}\right)\right), \quad i=1,2,3,
$$

encontradas no lema anterior.

Como $L_{r}\left(c_{1}\left(u_{3}\right), c_{2}\left(u_{3}\right), u_{3}\right)=0$ e $M_{r}\left(c_{1}\left(u_{3}\right), c_{2}\left(u_{3}\right), u_{3}\right)=0$ segue-se que

$$
\begin{aligned}
& \frac{\partial X_{1}}{\partial P}\left(c_{1}\left(u_{3}\right), c_{2}\left(u_{3}\right), u_{3}, P\right)=2 L_{r}\left(c_{1}\left(u_{3}\right), c_{2}\left(u_{3}\right), u_{3}\right)=0 \\
& \frac{\partial X_{2}}{\partial P}=4 L_{r} P+M_{r} \Rightarrow \frac{\partial X_{2}}{\partial P}\left(c_{1}\left(u_{3}\right), c_{2}\left(u_{3}\right), u_{3}, P_{i}\right)=0 \\
& \frac{\partial X_{3}}{\partial P}=2 L_{r}(\mathcal{U}+P \mathcal{V})+\left(2 L_{r} P+M_{r}\right) \mathcal{V} \Rightarrow \frac{\partial X_{3}}{\partial P}\left(c_{1}\left(u_{3}\right), c_{2}\left(u_{3}\right), u_{3}, P_{i}\right)=0,
\end{aligned}
$$


disto

$$
D X\left(\beta_{i}\left(u_{3}\right)\right)=\left(\begin{array}{cccc}
\frac{\partial X_{1}}{\partial u_{1}} & \frac{\partial X_{1}}{\partial u_{2}} & \frac{\partial X_{1}}{\partial u_{3}} & 0 \\
\frac{\partial X_{2}}{\partial u_{1}} & \frac{\partial X_{2}}{\partial u_{2}} & \frac{\partial X_{2}}{\partial u_{3}} & 0 \\
\frac{\partial X_{3}}{\partial u_{1}} & \frac{\partial X_{3}}{\partial u_{2}} & \frac{\partial X_{3}}{\partial u_{3}} & 0 \\
\frac{\partial X_{4}}{\partial u_{1}} & \frac{\partial X_{3}}{\partial u_{2}} & \frac{\partial X_{4}}{\partial u_{3}} & \frac{\partial X_{4}}{\partial P}
\end{array}\right) .
$$

Sendo $X_{1}=\mathcal{L}_{P}, X_{2}=P \mathcal{L}_{P}$ e $X_{3}=(\mathcal{U}+\mathcal{V} P) \mathcal{L}_{P}$, veja $(2.2)$, segue-se que

$$
\begin{aligned}
& \frac{\partial X_{1}}{\partial u_{j}}=\frac{\partial}{\partial u_{j}}\left(\mathcal{L}_{P}\right), \\
& \frac{\partial X_{2}}{\partial u_{j}}=P \frac{\partial}{\partial u_{j}}\left(\mathcal{L}_{P}\right), \\
& \frac{\partial X_{3}}{\partial u_{j}}=P \frac{\partial}{\partial u_{j}}\left(\mathcal{L}_{P}\right)(\mathcal{U}+P \mathcal{V})+\mathcal{L}_{P}\left(\frac{\partial \mathcal{U}}{\partial u_{j}}+P \frac{\partial \mathcal{V}}{\partial u_{j}}\right),
\end{aligned}
$$

para $j=1,2,3$. Como

$$
\mathcal{L}_{P}\left(\beta_{i}\left(u_{3}\right)\right)=2 L_{r}\left(c_{1}\left(u_{3}\right), c_{2}\left(u_{3}\right), u_{3}\right) P_{i}\left(u_{3}\right)+M_{r}\left(c_{1}\left(u_{3}\right), c_{2}\left(u_{3}\right), u_{3}\right)=0
$$

resulta que $\frac{\partial X_{i}}{\partial u_{j}}\left(\beta_{i}\left(u_{3}\right)\right)$ é múltiplo de $\frac{\partial}{\partial u_{j}}\left(\mathcal{L}_{P}\right)$ implicando que

$$
\operatorname{det}\left(\begin{array}{lll}
\frac{\partial X_{1}}{\partial u_{1}} & \frac{\partial X_{1}}{\partial u_{2}} & \frac{\partial X_{1}}{\partial u_{3}} \\
\frac{\partial X_{2}}{\partial u_{1}} & \frac{\partial X_{2}}{\partial u_{2}} & \frac{\partial X_{2}}{\partial u_{3}} \\
\frac{\partial X_{3}}{\partial u_{1}} & \frac{\partial X_{3}}{\partial u_{2}} & \frac{\partial X_{3}}{\partial u_{3}}
\end{array}\right)=0
$$

Usando (2.10), teremos que o polinômio característico de $D X\left(\beta_{i}\left(u_{3}\right)\right)$ é

$$
p(\lambda)=\lambda^{2} \cdot\left(\frac{\partial X_{4}}{\partial P}-\lambda\right) \cdot\left(\lambda-\frac{\partial X_{3}}{\partial u_{3}}-\frac{\partial X_{2}}{\partial u_{2}}-\frac{\partial X_{1}}{\partial u_{1}}\right)
$$

- Condição $D_{1}\left(\frac{a}{b}>\left(\frac{c}{2 b}\right)^{2}+2\right)$ :

Suponhamos $b>0$. Segue-se de $(2.11)$ e de (2.3) que os autovalores de $D X\left(\beta_{1}\left(u_{3}\right)\right)=$ $D X\left(c_{1}\left(u_{3}\right), c_{2}\left(u_{3}\right), u_{3}, P_{1}\left(u_{3}\right)\right)$ são

$$
\begin{aligned}
& \lambda_{1}\left(u_{3}\right) \equiv \lambda_{2}\left(u_{3}\right)=0, \\
& \lambda_{3}\left(u_{3}\right)=\frac{\partial X_{4}}{\partial P}=-2 b+a+O\left(u_{3}\right), \\
& \lambda_{4}\left(u_{3}\right)=\frac{\partial X_{1}}{\partial u_{1}}+\frac{\partial X_{2}}{\partial u_{2}}+\frac{\partial X_{3}}{\partial u_{3}}=b-a+O\left(u_{3}\right),
\end{aligned}
$$

Donde vemos que, para $u_{3}$ suficientemente pequeno, $\lambda_{3}>0$ e $\lambda_{4}<0$. Logo, teremos que $\beta_{1}\left(u_{3}\right)$ é uma curva normalmente hiperbólica do tipo sela. 
- Condição $D_{2}\left(1<\frac{a}{b}<\left(\frac{c}{2 b}\right)^{2}+2\right.$ e $\left.a \neq 2 b\right)$ :

Neste caso, $D X\left(\beta_{1}\left(u_{3}\right)\right)$ tem autovalores $\lambda_{1}, \lambda_{2}, \lambda_{3}$ e $\lambda_{4}$ satisfazendo (2.12). Entretanto, no presente caso, se $u_{3}$ é suficientemente pequeno então

$$
\lambda_{4}\left(u_{3}\right)<0 \text { e }\left\{\begin{array}{l}
\lambda_{3}\left(u_{3}\right)>0 \text { se } a>2 b \\
\lambda_{3}\left(u_{3}\right)<0 \text { se } a<2 b
\end{array}\right.
$$

Suporemos que $a<2 b$ e $b>0$, os outros casos são análogos.

Para $i=2,3$ teremos que os autovalores $\lambda_{1}, \lambda_{2}, \lambda_{3}$ e $\lambda_{4}$ de $D X\left(\beta_{i}\left(u_{3}\right)\right)=$ $D X\left(c_{1}\left(u_{3}\right), c_{2}\left(u_{3}\right), u_{3}, P_{i}\left(u_{3}\right)\right)$ satisfazem $\lambda_{1}=\lambda_{2} \equiv 0, \lambda_{3}>0$ e $\lambda_{4}<0$ já que, por (2.11) e (2.3), teremos que

$$
\begin{aligned}
\lambda_{3}\left(u_{3}\right) & =\frac{\partial X_{4}}{\partial P}\left(c_{1}\left(u_{3}\right), c_{2}\left(u_{3}\right), u_{3}, P_{i}\left(u_{3}\right)\right)=b P_{i}(0)^{2}+2 b-a+O(1)>0 \\
\lambda_{4}\left(u_{3}\right) & =\left(\frac{\partial X_{1}}{\partial u_{1}}+\frac{\partial X_{2}}{\partial u_{2}}+\frac{\partial X_{3}}{\partial u_{3}}\right)\left(c_{1}\left(u_{3}\right), c_{2}\left(u_{3}\right), u_{3}, P_{i}\left(u_{3}\right)\right)= \\
& =-b P_{i}(0)^{2}-b+O(1)<0
\end{aligned}
$$

Assim, concluimos que a condição $D_{2}$ implica que $\beta_{1}$ é normalmente hiperbólica do tipo atratora e $\beta_{2}, \beta_{3}$ são normalmente hiperbólicas do tipo sela. Como $a<2 b$ então $P_{2}\left(u_{3}\right)<0<P_{3}\left(u_{3}\right)$, para $u_{3}$ suficientemente pequeno, donde vemos que $\beta_{1}\left(u_{3}\right)$ está entre $\beta_{2}\left(u_{3}\right)$ e $\beta_{3}\left(u_{3}\right)$.

- Condição $D_{3}\left(\frac{a}{b}<1\right)$ :

Os autovalores de $D X\left(\beta_{i}\left(u_{3}\right)\right)$, para $i=1,2,3$, satisfazem

$$
\lambda_{1}\left(u_{3}\right)=\lambda_{2}\left(u_{3}\right) \equiv 0 \text { e } \lambda_{3}\left(u_{3}\right) \cdot \lambda_{4}\left(u_{3}\right)<0
$$

já que $\frac{a}{b}<2$, veja equação (2.14). Assim, para $u_{3}$ suficientemente pequeno, $\beta_{i}\left(u_{3}\right)$ é normalmente hiperbólica do tipo sela $(i=1,2,3)$.

Observação 7 Visto em $\mathbb{R}^{4}$, o campo de Lie-Cartan sempre tem um autovalor nulo. Nos interessa estudar $X$ restrito a hipersuperfície de Lie-Cartan.

Observação $8 \mathrm{Na}$ carta $\left(u_{1}, u_{2}, u_{3}, Q\right)$ o campo de Lie-Cartan é regular numa vizinahnça da origem. 
Passemos a análise da restrição do campo de Lie-Cartan, $X$, à hipersuperfície de Lie-Cartan, $\mathcal{L}^{-1}(0)$.

Na carta de Monge reduzida, escrevemos

$$
\begin{aligned}
& L_{r}=-b u_{2}-q_{111} u_{3}+O(2), \\
& M_{r}=(b-a) u_{1}+c u_{2}-\left(q_{201}-q_{021}\right) u_{3}+O(2), \\
& N_{r}=b u_{2}+q_{111} u_{3}+O(2) .
\end{aligned}
$$

Sendo $\mathcal{L}=L_{r} P^{2}+M_{r} P+N_{r}$ teremos que

$$
\begin{aligned}
\frac{\partial \mathcal{L}}{\partial u_{2}}\left(0,0,0, P_{i}(0)\right) & =\frac{\partial L_{r}}{\partial u_{2}}(0,0,0)\left(P_{i}(0)\right)^{2}+\frac{\partial M_{r}}{\partial u_{2}}(0,0,0) P_{i}(0)+\frac{\partial N_{r}}{\partial u_{2}}(0,0,0) \\
& =-b\left(P_{i}(0)\right)^{2}+c P_{i}(0)+b
\end{aligned}
$$

donde vemos que $\frac{\partial \mathcal{L}}{\partial u_{2}}\left(0,0,0, P_{i}(0)\right) \neq 0$, já que

$$
\frac{\partial \mathcal{L}}{\partial u_{2}}\left(0,0,0, P_{i}(0)\right)= \begin{cases}b, & \text { se } i=1 \\ a-b, & \text { se } i=2,3\end{cases}
$$

Disto, numa vizinhança de $\left(0,0,0, P_{i}(0)\right)(i=1,2,3)$, podemos escrever $u_{2}=u_{2}\left(u_{1}, u_{3}, P\right)$ na equação

$$
\mathcal{L}\left(u_{1}, u_{2}, u_{3}, P\right)=0 .
$$

A restrição do campo de Lie-Cartan à hipersuperfície de Lie-Cartan é dada por

$$
X_{\mathcal{L}}=\left\{\begin{array}{l}
\dot{u}_{1}=X_{1}\left(u_{1}, u_{2}\left(u_{1}, u_{3}, P\right), u_{3}, P\right) \\
\dot{u}_{3}=X_{3}\left(u_{1}, u_{2}\left(u_{1}, u_{3}, P\right), u_{3}, P\right) \\
\dot{P}=X_{4}\left(u_{1}, u_{2}\left(u_{1}, u_{3}, P\right), u_{3}, P\right)
\end{array}\right.
$$

A linearização de $X_{\mathcal{L}}$ possui um autovalor nulo e dois não nulos satisfazendo as mesmas condições dos autovalores não nulos, $\lambda_{3}$ e $\lambda_{4}$, dadas em (2.12), (2.13), (2.14) e (2.15). O autovalor nulo é associado ao vetor tangente à curva de equilíbrios. Disto, com relação a $X_{\mathcal{L}}$, concluimos que

Condição $\left.D_{1}\right) \Rightarrow \beta_{1}\left(u_{3}\right)$ é normalmente hiperbólica do tipo sela, Condição $\left.D_{2}\right) \Rightarrow$ se $a<2 b$ e $b>0$ teremos que, para $u_{3}$ suficientemente pequeno, a curva $\beta_{1}\left(u_{3}\right)$ é normalmente hiperbólica do tipo atratora, as curvas $\beta_{2}\left(u_{3}\right)$ e $\beta_{3}\left(u_{3}\right)$ são normalmente hiperbólicas do tipo sela, os outros casos são análogos.

Condição $\left.D_{3}\right) \Rightarrow$ as curvas $\beta_{i}\left(u_{3}\right)(i=1,2,3)$ são normalmente hiperbólicas do tipo sela. 
A seguir provaremos o teorema 1 enunciado no início desta seção.

Prova do teorema 1:

Seja c uma curva parcialmente umbílica Darbouxiana. Como visto em (2.1), podemos escrever $c$ da forma

$$
c\left(u_{3}\right)=\left(c_{1}\left(u_{3}\right), c_{2}\left(u_{3}\right), u_{3}\right) .
$$

- Suponhamos $c$ do tipo $D_{1}$.

Pelo lema 1, exite uma única curva de equilíbrios, $\beta_{1}\left(u_{3}\right)$, do campo de Lie-Cartan restrito a hipersuperfície de Lie-Cartan.

Pelo lema $2, \beta_{1}\left(u_{3}\right)$ é normalmente hiperbólica do tipo sela.

Assim, pelo teorema 4 enunciado no apêndice deste capítulo, existem únicas variedades invariantes, numa vizinhança $V_{\beta_{1}\left(u_{3}\right)}$ de $\beta_{1}\left(u_{3}\right)$, bidimensionais $W_{\beta_{1}}^{s}$ e $W_{\beta_{1}}^{u}$, de classe $C^{k-2}$, com $W_{\beta_{1}}^{u} \cap W_{\beta_{1}}^{s}=\beta_{1}\left(u_{3}\right)$.

Afirmação: $\Pi\left(W_{\beta_{1}}^{u}\right)=\left(c_{1}\left(u_{3}\right), c_{2}\left(u_{3}\right), u_{3}\right)$, onde $\Pi\left(u_{1}, u_{2}, u_{3}, P\right)=\left(u_{1}, u_{2}, u_{3}\right)$. De fato, como

- o eixo $P$ é invariante por $X$

- e, por $(2.9),(0,0,0,1)$ é o autovetor associado ao autovalor $\frac{\partial X_{4}}{\partial P}\left(\beta_{1}\left(u_{3}\right)\right)$ que é positivo para $u_{3}$ suficientemente pequeno, veja (2.12),

segue-se, pela unicidade das variedades invariantes, que numa vizinhança tubular de $\beta_{1}\left(u_{3}\right)$

$$
W_{\beta_{1}}^{u}=\left\{\left(c\left(u_{3}\right), P\right), \text { para } u_{3} \text { suficientemente pequeno. }\right\}
$$

resultando que $\Pi\left(W_{\beta_{1}}^{u}\right)=c\left(u_{3}\right)$.

Fazendo

$$
V_{c}=\Pi\left(V_{\beta_{1}\left(u_{3}\right)}\right) \text { e } W_{c}=\Pi\left(W_{\beta_{1}}^{s}\right),
$$

concluimos que: Se $c$ é do tipo $D_{1}$ então

- existe uma única superfície separatriz parcialmente umbílica, $W_{c}$, de classe $C^{k-2}$, 
- por vi) do teorema 4 no apêndice deste capítulo, $W_{c}$ é fibrada sobre $c$ e estas fibras são folhas de $\mathcal{F}_{1}(\alpha)$,

- existe uma vizinhança tubular $V_{c}$ de $c$ tal que o conjunto $V_{c} \backslash W_{c}$ é um setor hiperbólico de $\mathcal{F}_{1}(\alpha)$.

Veja figura 2.6.

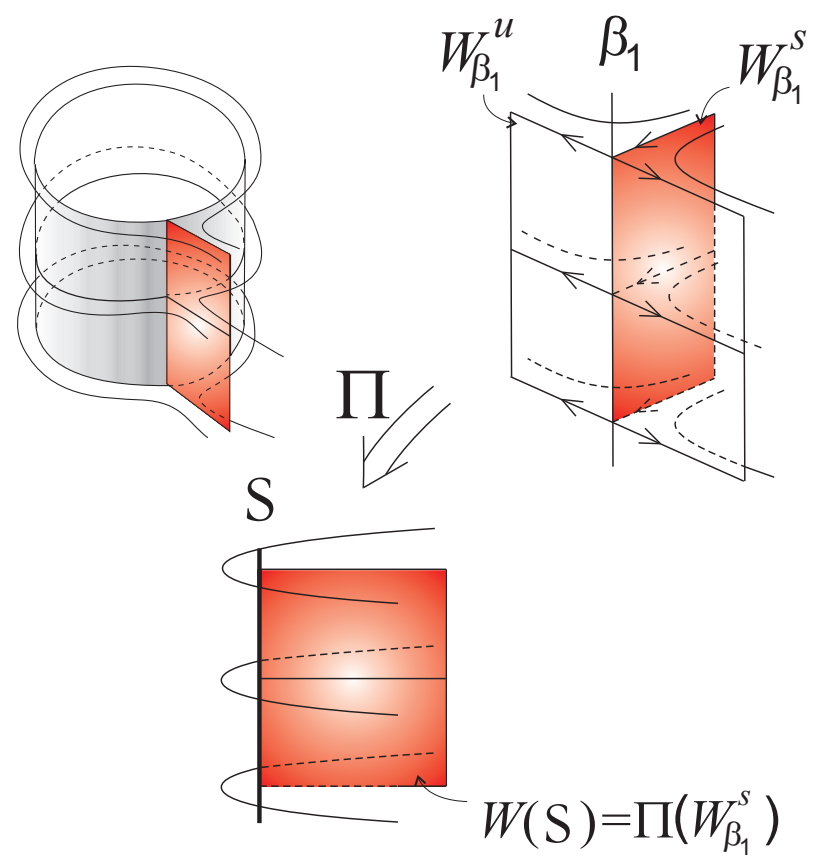

Figura 2.6: Projeção para o caso $D_{1}$. 
- Suponhamos $c$ do tipo $D_{2}$.

Pelo lema 1, existem exatamente três curvas de equilíbrios, $\beta_{1}\left(u_{3}\right), \beta_{2}\left(u_{3}\right)$ e $\beta_{3}\left(u_{3}\right)$, do campo de Lie-Cartan restrito a hipersuperfície de Lie-Cartan.

Pelo lema $2, \beta_{1}\left(u_{3}\right)$ é normalmente hiperbólica do tipo atratora, $\beta_{2}\left(u_{3}\right)$ e $\beta_{3}\left(u_{3}\right)$ são normalmente hiperbólicas do tipo sela.

Assim, pelo teorema 4, teremos que

- Para $i=2,3$, existem únicas variedades invariantes bidimensionais $W_{\beta_{i}}^{s}$ e $W_{\beta_{i}}^{u}$, de classe $C^{k-2}, \operatorname{com} W_{\beta_{i}}^{u} \cap W_{\beta_{i}}^{s}=\beta_{i}\left(u_{3}\right)$.

- Para $\beta_{1}\left(u_{3}\right), W_{\beta_{i}}^{u}=\emptyset$

Como no caso $D_{1}$, neste caso, teremos que $\Pi\left(W_{\beta_{1}}^{s}\right)=\Pi\left(W_{\beta_{i}}^{u}\right)=\left(c_{1}\left(u_{3}\right), c_{2}\left(u_{3}\right), u_{3}\right)$, $i=1,2$.

Fazendo

$$
W_{1}=\Pi\left(W_{\beta_{2}}^{s}\right) \text { e } W_{2}=\Pi\left(W_{\beta_{3}}^{s}\right),
$$

concluimos que: Se $c$ é do tipo $D_{2}$ então

- existem duas superfície separatriz parcialmente umbílica, $W_{1}$ e $W_{2}$, ambas de classe $C^{k-2}$,

- existe exatamente um setor hiperbólico e uma setor parabólico de $\mathcal{F}_{1}(\alpha)$.

Veja figura 2.7 


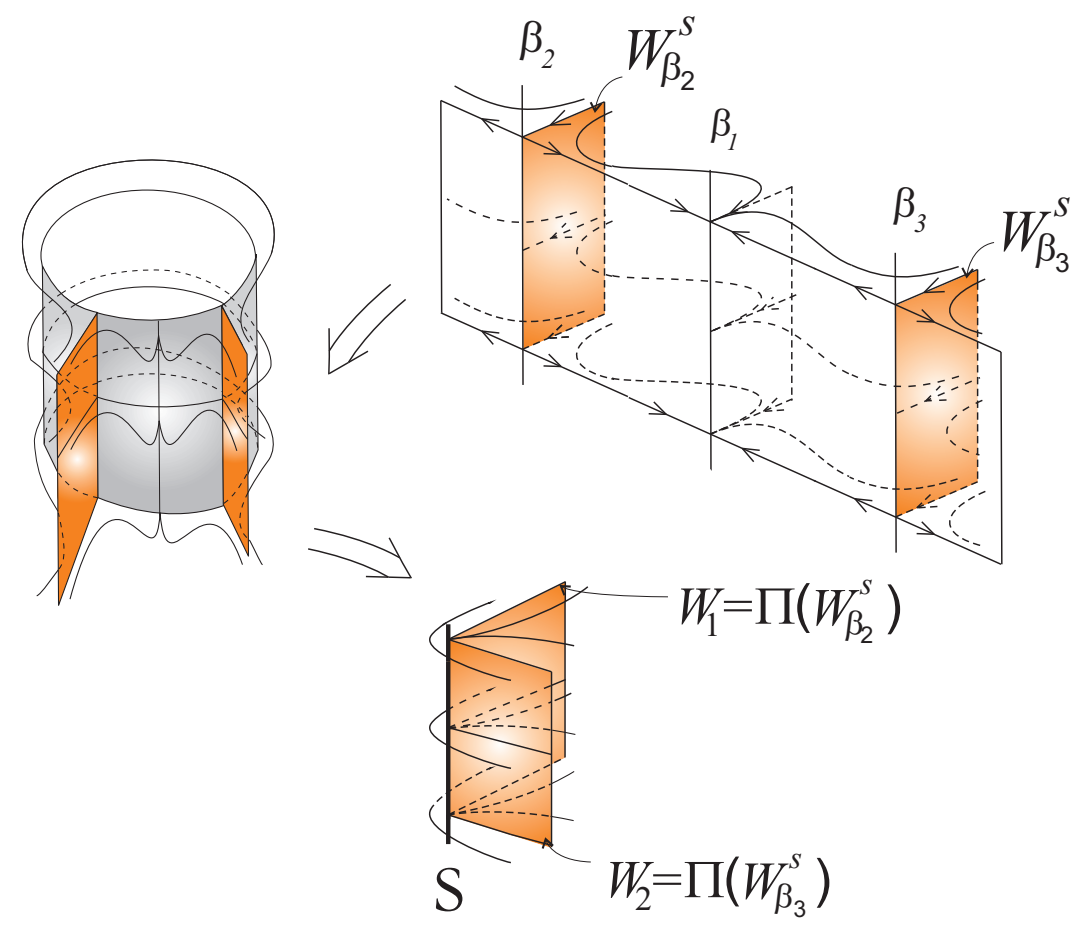

Figura 2.7: Projeção para o caso $D_{2} \operatorname{com} \frac{a}{b}<2$.

- Suponhamos $c$ do tipo $D_{3}$.

Pelo lema 1 , existem exatamente três curvas de equilíbrios, $\beta_{1}\left(u_{3}\right), \beta_{2}\left(u_{3}\right)$ e $\beta_{3}\left(u_{3}\right)$, para o campo de Lie-Cartan restrito a hipersuperfície de Lie-Cartan.

Pelo lema 2, as curvas $\beta_{1}\left(u_{3}\right), \beta_{2}\left(u_{3}\right)$ e $\beta_{3}\left(u_{3}\right)$ são normalmente hiperbólicas do tipo sela.

Assim, do teorema 4, teremos que para $i=1,2,3$, existem únicas variedades invariantes bidimensionais $W_{\beta_{i}}^{s}$ e $W_{\beta_{i}}^{u}$, de classe $C^{k-2}$, com $W_{\beta_{i}}^{u} \cap W_{\beta_{i}}^{s}=\beta_{i}$. Além disso,

$$
\Pi\left(W_{\beta_{1}}^{s}\right)=\Pi\left(W_{\beta_{i}}^{u}\right)=\left(c_{1}\left(u_{3}\right), c_{2}\left(u_{3}\right), u_{3}\right), i=2,3 .
$$

Fazendo

$$
W_{1}=\Pi\left(W_{\beta_{1}}^{u}\right), W_{2}=\Pi\left(W_{\beta_{2}}^{s}\right) \text { e } W_{3}=\Pi\left(W_{\beta_{3}}^{s}\right),
$$

concluimos que: Se $c$ é do tipo $D_{3}$ então

- existem três superfície separatrizes parcialmente umbílica, $W_{1}, W_{2}$ e $W_{3}$, todas de classe $C^{k-2}$, 
- existe exatamente três setores hiperbólicos de $\mathcal{F}_{1}(\alpha)$.

Veja figura 2.8.
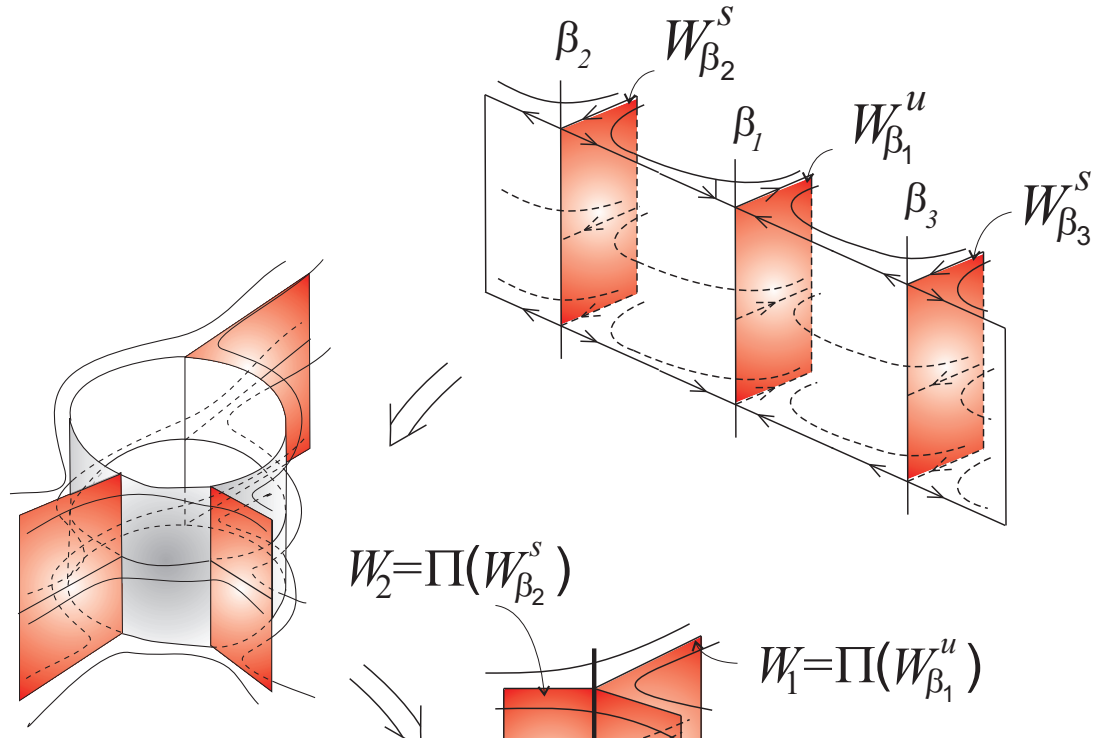

$W_{2}=\Pi\left(W_{\beta_{2}}^{s}\right)$

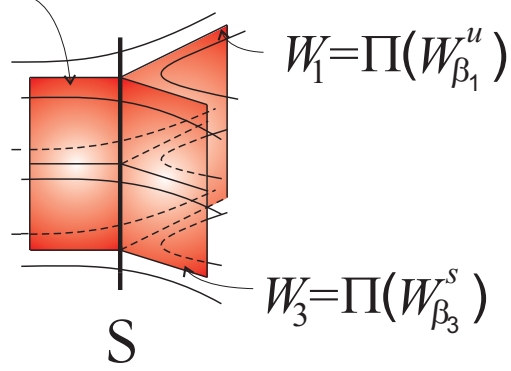

Figura 2.8: Projeção para o caso $D_{3}$. 


\subsection{Curva Parcialmente Umbílica em torno de um ponto do tipo $D_{23}$}

Nesta seção, provaremos o seguinte teorema:

Teorema 2 (R. Garcia, [6]) Suponhamos $\alpha \in \mathcal{I}^{k}\left(\mathbb{M}^{3}, \mathbb{R}^{4}\right), k \geq 4$, e p um ponto parcialmente umbílico de $\alpha$ do tipo $D_{23}$. Então o comportamento das linhas de curvatura numa vizinhança de p é como na figura 2.9.

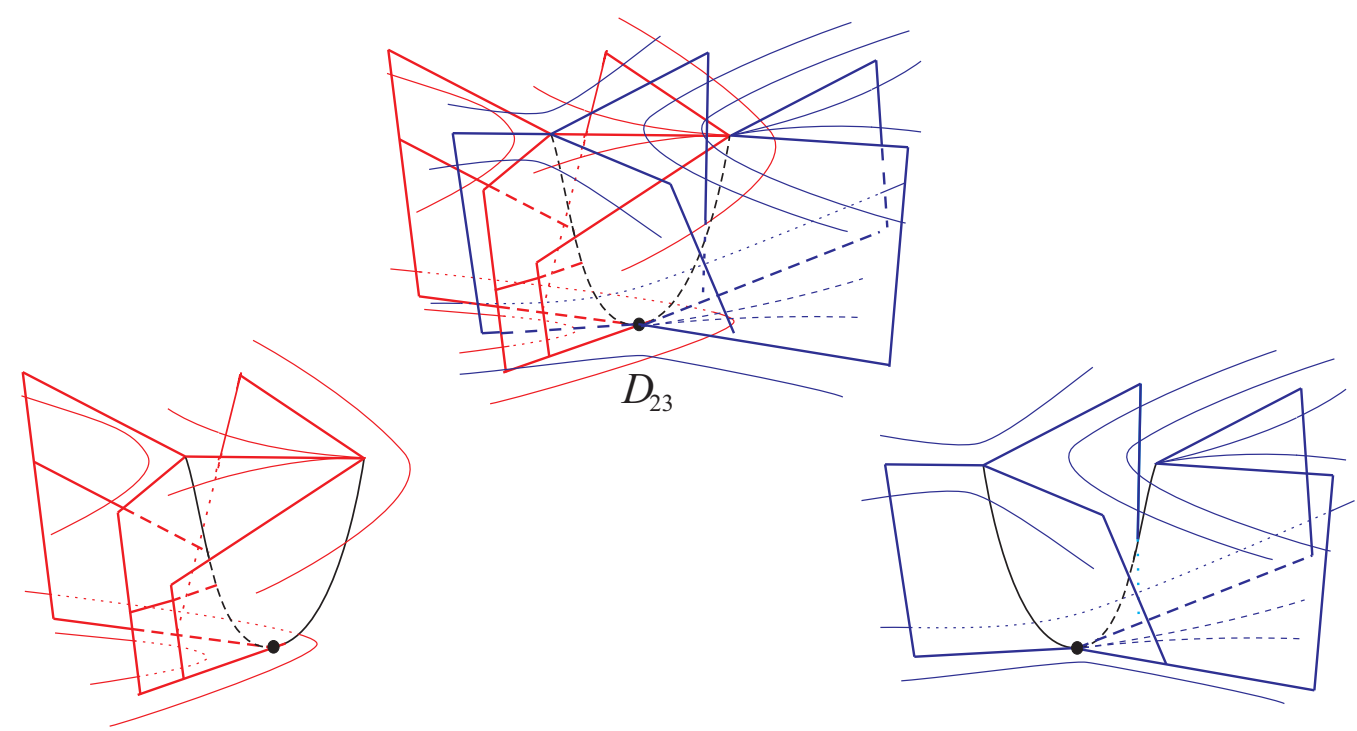

Figura 2.9: A figura do meio representa a configuração principal de $\alpha$ numa vizinhança de um ponto do tipo $D_{23}$, e as das laterais representam cada folheação $\mathcal{F}_{1}$ e $\mathcal{F}_{2}$ separadamente.

Observação 9 Dividiremos a demonstração do teorema 2 em 3 lemas, dados ao longo desta seção.

Escrevemos a imersão $\alpha$ na carta de Monge reduzida:

$$
\alpha\left(u_{1}, u_{2}, u_{3}\right)=\left(u_{1}, u_{2}, u_{3}, h\left(u_{1}, u_{2}, u_{3}\right)\right)
$$


onde

$$
\begin{aligned}
h\left(u_{1}, u_{2}, u_{3}\right) & =\frac{k}{2}\left(u_{1}^{2}+u_{2}^{2}\right)+\frac{k_{3}}{2} u_{3}^{2}+\frac{1}{6} a u_{1}^{3}+\frac{1}{2} b u_{1} u_{2}^{2}+\frac{1}{6} c u_{2}^{3}+\frac{1}{6} q_{003} u_{3}^{3} \\
& +\frac{1}{2} q_{012} u_{2} u_{3}^{2}+q_{111} u_{1} u_{2} u_{3}+\frac{1}{2} q_{021} u_{2}^{2} u_{3}+\frac{1}{2} q_{102} u_{1} u_{3}^{2} \\
& +\frac{1}{2} q_{201} u_{1}^{2} u_{3}+\frac{1}{24} A u_{1}^{4}+\frac{1}{6} B u_{1}^{3} u_{2}+\frac{1}{4} C u_{1}^{2} u_{2}^{2}+\frac{1}{6} D u_{1} u_{2}^{3} \\
& +\frac{1}{24} E u_{2}^{4}+\frac{1}{24} Q_{004} u_{3}^{4}+\frac{1}{6} Q_{013} u_{2} u_{3}^{3}+\frac{1}{6} Q_{103} u_{1} u_{3}^{3}+\frac{1}{4} Q_{022} u_{2}^{2} u_{3}^{2} \\
& +\frac{1}{4} Q_{202} u_{1}^{2} u_{3}^{2}+\frac{1}{2} Q_{112} u_{1} u_{2} u_{3}^{2}+\frac{1}{6} Q_{031} u_{2}^{3} u_{3}+\frac{1}{6} Q_{301} u_{1}^{3} u_{3} \\
& +\frac{1}{2} Q_{121} u_{1} u_{2}^{2} u_{3}+\frac{1}{2} Q_{211} u_{1}^{2} u_{2} u_{3}+O(5) .
\end{aligned}
$$

Nesta carta com $k_{3}=1$, vimos na definição 2 no capítulo 1 que as condições para que $p$ seja do tipo $D_{23}$ são:

1) $b=a \neq 0,-b\left(-q_{201}+q_{021}\right)-c q_{111} \neq 0 \mathrm{e}$

2) $\chi=c B-\left(C-A+2 k^{3}\right) b+\frac{-2 q_{111}^{2} b+2 q_{201}^{2} b+2 q_{201} q_{111} c}{b(k-1)} \neq 0$

Como veremos no lema 3 a condição 1) implicará que $p$ pertence a uma curva regular de pontos parcialmente umbílicos. Já a condição 2) será usada para mostrar que ocorre a transição $D_{2}-D_{3}$ ao longo da curva parcialmente umbílica, veja lema 5 .

Lema 3 Se $p$ é um ponto parcialmente umbílico, de $\alpha$, semi-Darbouxiano do tipo $D_{23}$ então p pertente a uma curva regular c de pontos parcialmente umbílicos. Além disso, numa vizinhança de $p$, podemos escrever as coordenadas $u_{1}, u_{2}, u_{3}$ de c da seguinte forma:

$$
\begin{aligned}
u_{2}=c_{2}\left(u_{1}\right) & =-\frac{1}{2\left(\left(q_{021}-q_{201}\right) b-c q_{111}\right)}\left[q_{111} A+q_{111} C-\left(q_{021}-q_{201}\right) B\right. \\
& \left.-2\left(q_{201} q_{021}-q_{111}^{2}\right) q_{111}(k-1)^{-1}-2 q_{111} k^{3}\right] u_{1}^{2}+O(3) \\
u_{3}=c_{3}\left(u_{1}\right)= & \frac{1}{\left(-2 q_{201} b-2 c q_{111}+42 b\right)}\left[b A-b C+c B-2 k^{3} b\right. \\
& \left.+(k-1)^{-1}\left(-2\left(q_{111}^{2}+q_{201}^{2}\right) b+2 q_{201} q_{111} c\right)\right] u_{1}^{2}+O(3)
\end{aligned}
$$


Demonstração: Vimos no capítulo 1, observação 5, que os pontos parcialmente umbílicos são dados por

$$
L_{r}\left(u_{1}, u_{2}, u_{3}\right)=0, \quad M_{r}\left(u_{1}, u_{2}, u_{3}\right)=0
$$

e, de (1.15),

$$
\operatorname{det}\left(\left.\frac{\partial\left(L_{r}, M_{r}\right)}{\partial\left(u_{2}, u_{3}\right)}\right|_{u_{1}=0, u_{2}=0, u_{3}=0}\right)=b\left(-q_{201}+q_{021}\right)+c q_{111} \neq 0,
$$

implicando, pelo teorema da função implícita, que $p$ pertence a uma curva regular de pontos parcialmente umbílicos e que, além disso, podemos escrever $u_{2}=c_{2}\left(u_{1}\right)$ e $u_{3}=c_{3}\left(u_{1}\right)$ em (2.19). Para encontrar o segundo jato de $c_{2}$ e $c_{3}$, escrevemos

$$
\begin{aligned}
& c_{2}\left(u_{1}\right)=c_{2}^{1} u_{1}+c_{2}^{2} u_{1}^{2} \\
& c_{3}\left(u_{1}\right)=c_{3}^{1} u_{1}+c_{3}^{2} u_{1}^{2} .
\end{aligned}
$$

Resolvendo

$$
\left\{\begin{aligned}
\frac{\partial}{\partial u_{1}}\left[L_{r}\left(u_{1}, c_{2}\left(u_{1}\right), c_{3}\left(u_{1}\right)\right)\right] & =0 \\
\frac{\partial}{\partial u_{1}}\left[M_{r}\left(u_{1}, c_{2}\left(u_{1}\right), c_{3}\left(u_{1}\right)\right)\right] & =0 \\
\frac{\partial^{2}}{\partial u_{1}^{2}}\left[L_{r}\left(u_{1}, c_{2}\left(u_{1}\right), c_{3}\left(u_{1}\right)\right)\right] & =0 \\
\frac{\partial^{2}}{\partial u_{1}^{2}}\left[M_{r}\left(u_{1}, c_{2}\left(u_{1}\right), c_{3}\left(u_{1}\right)\right)\right] & =0
\end{aligned}\right.
$$

em $c_{2}^{1}, c_{2}^{2}, c_{3}^{1}$ e $c_{3}^{2}$ obtemos que

$$
\begin{aligned}
c_{2}\left(u_{1}\right) & =-\frac{1}{2\left(\left(q_{021}-q_{201}\right) b-c q_{111}\right)}\left[q_{111} A+q_{111} C-\left(q_{021}-q_{201}\right) B\right. \\
& \left.-2\left(q_{201} q_{021}-q_{111}^{2}\right) q_{111}(k-1)^{-1}-2 q_{111} k^{3}\right] u_{1}^{2}+O(3) \\
c_{3}\left(u_{1}\right) & =\frac{1}{\left(-2 q_{201} b-2 c q_{111}+42 b\right)}\left[b A-b C+c B-2 k^{3} b\right. \\
& \left.+(k-1)^{-1}\left(-2\left(q_{111}^{2}+q_{201}^{2}\right) b+2 q_{201} q_{111} c\right)\right] u_{1}^{2}+O(3)
\end{aligned}
$$


Na seção anterior, vimos que a configuração principal em torno de uma curva parcialmente umbílica do tipo $D_{3}$ é proveniente da projeção das curvas integrais de $X_{\mathcal{L}}$ numa vizinhança de três selas normalmente hiperbólicas, e a configuração principal numa vizinhança de uma curva do tipo $D_{2}$ é proveniente da projeção das curvas integrais de $X_{\mathcal{L}}$ numa vizinhança de um nó normalmente hiperbólico entre duas selas normalmente hiperbólicas. Assim, devemos mostrar que existem três curvas de equilíbrios de $X$, sendo duas do tipo sela e uma do tipo sela-nó, ver figura abaixo:

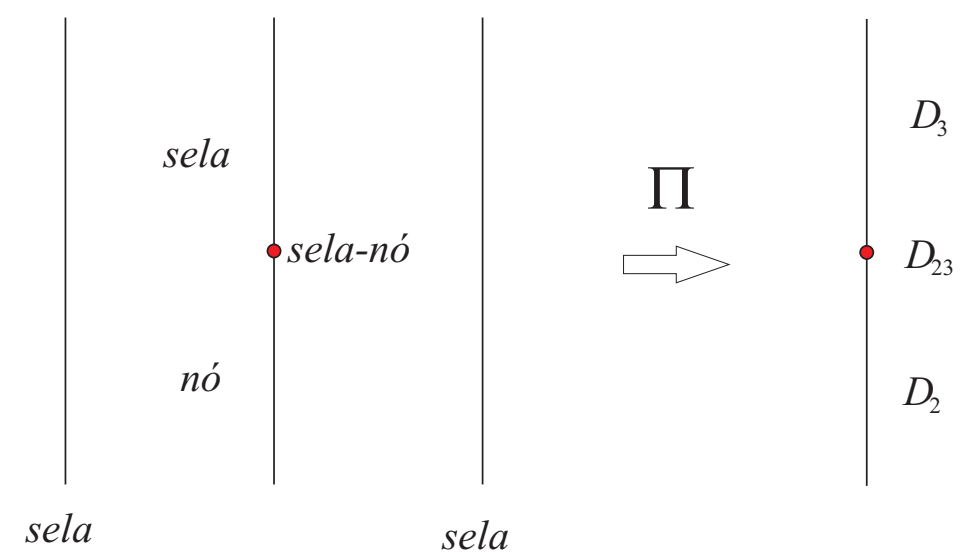

Figura 2.10: Equilíbrios de $X$ associados a um ponto parcialmente umbílico do tipo $D_{23}$ 
O lema 4 a seguir garante a existência das três curvas de equilíbrios, e o lema 5 mostra a estrutura da curvas integrais de $X$ numa vizinhança de tais curvas de equilíbrios.

Lema 4 Sejam $\left(u_{1}, u_{2}, u_{3}\right): \mathbb{M}^{3} \rightarrow \mathbb{R}^{3}$ a carta de Monge reduzida com $k_{3}=1, X$ o campo dado em (2.2) e $c\left(u_{1}\right)=\left(u_{1}, c_{2}\left(u_{1}\right), c_{3}\left(u_{1}\right)\right)$ uma curva parcialmente umbílica tal que $p=c(0)$ é do tipo $D_{23}$. Para $u_{1}$ suficientemente pequeno, o conjunto dos equilíbrios de $X$, que se projetam em c, é formado por três curvas regulares $\beta_{i}\left(u_{1}\right), i=1,2,3$, satisfazendo

$$
\beta_{i}\left(u_{1}\right)=\left(u_{1}, c_{2}\left(u_{1}\right), c_{3}\left(u_{1}\right), P_{i}\left(u_{1}\right)\right)
$$

onde

$$
\begin{aligned}
& P_{1}\left(u_{1}\right)=0+O(1) ; \\
& P_{2}\left(u_{1}\right)=\frac{c+\sqrt{c^{2}+4 b^{2}}}{2 b}+O(1) ; \\
& P_{3}\left(u_{1}\right)=\frac{c-\sqrt{c^{2}+4 b^{2}}}{2 b}+O(1) .
\end{aligned}
$$

Demonstração: Sendo

$$
X=\left\{\begin{array}{l}
\dot{u}_{1}=\mathcal{L}_{P} \\
\dot{u}_{2}=P \mathcal{L}_{P} \\
\dot{u}_{3}=(\mathcal{U}+\mathcal{V} P) \mathcal{L}_{P} \\
\dot{P}=-\left(\mathcal{L}_{u_{1}}+P \mathcal{L}_{u_{2}}+\mathcal{L}_{u_{3}}(\mathcal{U}+\mathcal{V} P)\right)
\end{array}\right.
$$

segue-se que os equilíbrios de $X$ são dados por

$$
\left\{\begin{array}{l}
\mathcal{L}_{P}\left(u_{1}, u_{2}, u_{3}, P\right)=0 \\
\left(\mathcal{L}_{u_{1}}+P \mathcal{L}_{u_{2}}+\mathcal{L}_{u_{3}}(\mathcal{U}+\mathcal{V} P)\right)\left(u_{1}, u_{2}, u_{3}, P\right)=0
\end{array}\right.
$$

Como

$$
\mathcal{L}_{P}\left(u_{1}, u_{2}, u_{3}\right)=2 P L_{r}\left(u_{1}, u_{2}, u_{3}\right)+M_{r}\left(u_{1}, u_{2}, u_{3}\right),
$$

resulta que os pontos parcialmente umbílicos, que neste caso formam uma curva regular $c$, anulam a primeira equação de (2.21). Pelo lema (3) podemos escrever as coordenadas de $c$ da forma

$$
u_{2}=c_{2}\left(u_{1}\right), \quad u_{3}=c_{3}\left(u_{1}\right),
$$


e, além disso,

$$
\begin{aligned}
c_{2}\left(u_{1}\right) & =-\frac{1}{2\left(\left(q_{021}-q_{201}\right) b-c q_{111}\right)}\left[q_{111} A+q_{111} C-\left(q_{021}-q_{201}\right) B\right. \\
& \left.-2\left(q_{201} q_{021}-q_{111}^{2}\right) q_{111}(k-1)^{-1}-2 q_{111} k^{3}\right] u_{1}^{2}+O(3) \\
c_{3}\left(u_{1}\right)= & \frac{1}{\left(-2 q_{201} b-2 c q_{111}+42 b\right)}\left[b A-b C+c B-2 k^{3} b\right. \\
& \left.+(k-1)^{-1}\left(-2\left(q_{111}^{2}+q_{201}^{2}\right) b+2 q_{201} q_{111} c\right)\right] u_{1}^{2}+O(3)
\end{aligned}
$$

Substituindo (2.22) e (2.23) na segunda equação de (2.4) e fazendo $a=b$ em (2.3) obtemos a seguinte equação cúbica em $P$ :

$$
(b+O(1)) P^{3}+(-c+O(1)) P^{2}+(-b+O(1)) P+O(1)=0,
$$

cujo discriminante é dado por

$$
D\left(u_{1}\right)=-\frac{1}{108} \frac{c^{2}+4 b^{2}}{b^{2}}+O(1)<0 .
$$

Disto, para $u_{1}$ suficientemente pequeno, a equação $(2.24)$ possui três soluções $P_{i}\left(u_{1}\right)(i=$ $1,2,3)$ como dado em (2.20), já que em $u_{1}=0$ a cúbica (2.24) tem as seguintes raízes:

$$
\begin{aligned}
& P_{1}(0)=0 \\
& P_{2}(0)=\frac{1}{2} \frac{c+\sqrt{c^{2}+4 b^{2}}}{b} \\
& P_{3}(0)=\frac{1}{2} \frac{c-\sqrt{c^{2}+4 b^{2}}}{b}
\end{aligned}
$$

Resumindo, mostramos que $X$ possui três curvas de equilíbrios $\beta_{1}, \beta_{2}$ e $\beta_{3}$ tais que $\Pi\left(\beta_{i}\right)=c, i=1,2,3$. No espaço das variáveis $u_{1}, u_{2}, u_{3}$ e $P$ escrevemos as curvas de equilíbrios de $X$ como segue:

$$
\beta_{i}: \quad u_{2}=c_{2}\left(u_{1}\right), u_{3}=c_{3}\left(u_{1}\right) \text { e } P=P_{i}\left(u_{1}\right), \quad i=1,2,3
$$

onde $c_{2}, c_{3}$ e $P_{i}(i=1,2,3)$ foram dadas em (2.18) e (2.20) respectivamente.

Lema 5 Seja $X_{\mathcal{L}}$ o campo de Lie-Cartan restrito a hipersuperfície de Lie-Cartan. As curvas $\beta_{i}, i=2,3$ são normalmente hiperbólicas do tipo sela para $X_{\mathcal{L}}$. Quanto a curva $\beta_{1}$, o retrato de fase de $X_{\mathcal{L}}$ é como na figura 2.11 abaixo. 


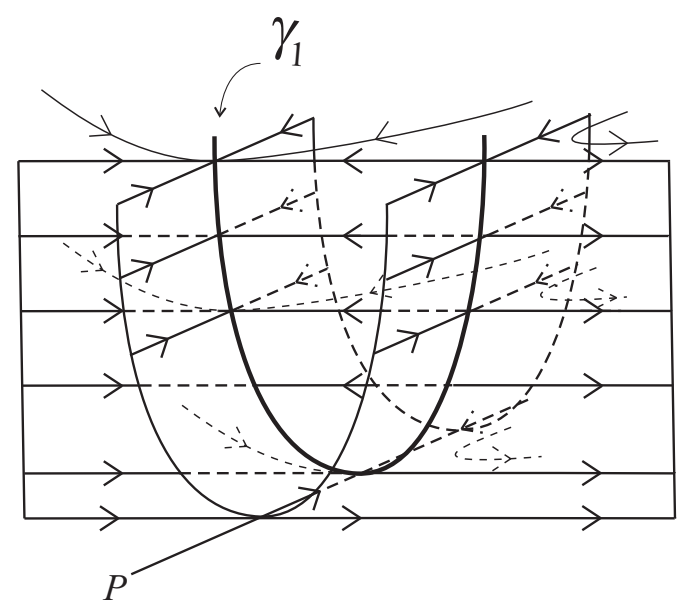

Figura 2.11: Retrato de fase do campo de Lie-Cartan restrito a hipersuperfície de Lie-Cartan, numa vizinhança da curva de equilíbrios $\beta_{1}$.

Demonstração: A linearização $D X\left(\beta_{i}\left(u_{1}\right)\right), i=2,3$, de $(2.2)$, possui dois autovalores identicamente nulos e os seguintes dois não nulos:

$$
\lambda_{1}\left(u_{1}\right)=-b\left(P_{i}(0)^{2}+1\right)+O(1), \quad \lambda_{2}\left(u_{1}\right)=b\left(P_{i}(0)^{2}+1\right)+O(1), \quad i=2,3 .
$$

Disto, para $u_{1}$ suficientemente pequeno, $\lambda_{1}\left(u_{1}\right)<0$ e $\lambda_{2}\left(u_{1}\right)>0$, donde vemos que, restrito à hipersuperfície de Lie-Cartan, $\beta_{2}$ e $\beta_{3}$ são normalmente hiperbólicas do tipo sela.

Observação 10 - Visto em $\mathbb{R}^{4}$ o campo de Lie-Cartan sempre possui um autovalor nulo, este autovalor desaparece quando feita a restrição de $X$ a hipersuperfície de Lie-Cartan. O outro autovalor nulo corresponde à direção tangente a curva de equilibrios.

- Numa vizinhança de $P=P_{i}(0), i=2,3$, podemos escrever a hipersuperfície de Lie-Cartan como gráfico nas variáveis $u_{1}, u_{2}$ e $P$, já que

$$
\frac{\partial \mathcal{L}}{\partial u_{3}}\left(0,0,0, P_{i}(0)\right)=-\frac{\left(b\left(q_{021}-q_{201}\right)-c q_{111}\right)\left(c+(-1)^{i} \sqrt{c^{2}+4 b^{2}}\right)}{2 b^{2}} \cdot(i=2,3)
$$

Passemos ao estudo de $X$, restrito a hipersuperfície de Lie-Cartan, numa vizinhança de $\beta_{1}\left(u_{1}\right)$, para $u_{1}$ suficientemente pequeno. 
Seja $\mathcal{L}\left(u_{1}, u_{2}, u_{3}, P\right)=0$ a equação que define a hipersuperfície de Lie-Cartan, onde

$$
\mathcal{L}\left(u_{1}, u_{2}, u_{3}, P\right)=L_{r}\left(u_{1}, u_{2}, u_{3}\right) P^{2}+M_{r}\left(u_{1}, u_{2}, u_{3}\right) P+N_{r}\left(u_{1}, u_{2}, u_{3}\right) .
$$

Na carta de Monge reduzida, temos que

$$
\begin{aligned}
& L_{r}=-b u_{2}-q_{111} u_{3}+O(2), \\
& M_{r}=c u_{2}-\left(q_{201}-q_{021}\right) u_{3}+O(2), \\
& N_{r}=b u_{2}+q_{111} u_{3}+O(2) .
\end{aligned}
$$

Assim,

$$
\frac{\partial \mathcal{L}}{\partial u_{2}}(0,0,0,0)=b \neq 0
$$

implicando, pelo teorema da função implícita, que podemos escrever $u_{2}=u_{2}\left(u_{1}, u_{3}, P\right)$ na equação $\mathcal{L}\left(u_{1}, u_{2}, u_{3}, P\right)=0$, numa vizinhança da origem. A expansão em Taylor, até segunda ordem, de $u_{2}\left(u_{1}, u_{3}, P\right)$ é a seguinte

$$
\begin{aligned}
u_{2}\left(u_{1}, u_{3}, P\right) & =-\frac{q_{111}}{b} u_{3}-\frac{1}{2 b}\left(B+2 \frac{q_{201} q_{111}}{k-1}\right) u_{1}^{2}+\left(\frac{q_{201}}{b}-\frac{q_{021}}{b}+\frac{c q_{111}}{b^{2}}\right) u_{3} P \\
& +\left(\frac{q_{201} q_{111} q_{021}}{(k-1) b^{2}}-\frac{q_{201} q_{012}}{(k-1) b}+\frac{q_{111}{ }^{3}}{(k-1) b^{2}}+\frac{q_{111} C}{b^{2}}-\frac{q_{102} q_{111}}{(k-1) b}\right. \\
& \left.-\frac{q_{111} k^{3}}{b^{2}}-\frac{Q_{211}}{b}\right) u_{1} u_{3}+\left(-\frac{q_{111}{ }^{3} q_{021}}{(k-1) b^{3}}+\frac{q_{111} q_{021} q_{102}}{(k-1) b^{2}}-\frac{1}{2} \frac{D q_{111}{ }^{2}}{b^{3}}\right. \\
& \left.+\frac{q_{111}^{2} q_{012}}{(k-1) b^{2}}+\frac{Q_{121} q_{111}}{b^{2}}-\frac{1}{2} \frac{Q_{112}}{b}-\frac{q_{012} q_{102}}{b(k-1)}\right) u_{3}^{2}+O(3)
\end{aligned}
$$

Substituindo $u_{2}=u_{2}\left(u_{1}, u_{3}, P\right)$ nas coordenadas de $X$ dadas em (2.3) com $a=b$, obtemos a restrição do campo de Lie-Cartan à hipersuperfície de Lie-Cartan:

$$
X_{\mathcal{L}}:=\left\{\begin{array}{l}
\left.\dot{u}_{1}=X_{1}\left(u_{1}, u_{2}\left(u_{1}, u_{3}, P\right), u_{3}, P\right)\right) \\
\dot{u}_{3}=X_{3}\left(u_{1}, u_{2}\left(u_{1}, u_{3}, P\right), u_{3}, P\right) \\
\dot{p}=X_{4}\left(u_{1}, u_{2}\left(u_{1}, u_{3}, P\right), u_{3}, P\right)
\end{array} .\right.
$$

onde

$$
\begin{aligned}
X_{1} & =\frac{\left(q_{021} b-c q_{111}-q_{201} b\right)}{b} u_{3}+O(2) \\
X_{3} & =\left(\mathcal{U}\left(u_{1}, u_{2}\left(u_{1}, u_{3}, P\right), u_{3}\right)+P \mathcal{V}\left(u_{1}, u_{2}\left(u_{1}, u_{3}, P\right), u_{3}\right)\right) X_{1} \\
X_{4} & =\left(-B-3 \frac{q_{201} q_{111}}{k-1}\right) u_{1}-b P+ \\
& +\left(-Q_{211}+\frac{q_{111} C}{b}+\frac{k^{3}}{b}-\frac{2 q_{102} q_{111}}{k-1}-\frac{q_{201} q_{012}}{k-1}+\frac{2 u_{3} q_{111}{ }^{3}}{(k-1) b}+\frac{q_{201} q_{021} q_{111}}{(k-1) b}\right) u_{3}- \\
& +O(2)
\end{aligned}
$$


Os autovalores de $D X_{\mathcal{L}}\left(\beta_{1}\left(u_{1}\right)\right)$ são

$$
\begin{aligned}
& \lambda_{1}\left(u_{1}\right) \equiv 0 \\
& \lambda_{2}\left(u_{1}\right)=-b+O(1) \\
& \lambda_{3}\left(u_{1}\right)=\frac{q_{201}\left(\left(q_{021}-q_{201}\right) b-c q_{111}\right)}{b(k-1)} u_{1}+O(2) .
\end{aligned}
$$

Observação 11 Seja $\epsilon>0$ suficientemente pequeno e suponha, sem perda de generalidade, que $\frac{q_{201}\left(\left(q_{021}-q_{201}\right) b-c q_{111}\right)}{b(k-1)}>0$ e $b>0$.

Se $\left|u_{1}\right|>\epsilon$, teremos que:

- $\beta_{1}\left(u_{1}\right)$ é normalmente hiperbólica do tipo sela, se $u_{1}>\epsilon$,

- $\beta_{1}\left(u_{1}\right)$ é normalmente hiperbólica do tipo atratora, se $u_{1}<-\epsilon$

Observação 12 Sendo $\lambda_{2}\left(u_{1}\right)<0$, para $u_{1}$ suficientemente pequeno, segue-se do teorema 4, no apêndice deste capítulo, que existe uma variedade, que denotaremos por $W_{\beta_{1}\left(u_{1}\right)}^{s}$, invariante por $X_{\mathcal{L}}$, de classe $C^{k-2}$ e onde $\beta_{1}\left(u_{1}\right)$ é atratora.

A variedade $W_{\beta_{1}\left(u_{1}\right)}^{s}$ é a variedade atratora na figura 2.11 .

Observação 13 Quando $u_{1}=0$, a variedade central em $\beta_{1}(0), W_{\beta_{1}(0)}^{c}$, tem dimensão 2 e contém a curva de equilíbrios, $\beta_{1}$, numa vizinhança de $\beta_{1}(0)$.

Como veremos, a variedade $W_{\beta_{1}(0)}^{c}$ é a variedade do tipo sela-nó na figura 2.11.

Passemos ao estudo de $X_{\mathcal{L}}$ restrito a $W_{\beta_{1}(0)}^{c}$. Para isto, faremos uma mudança de coordenadas, $\left(u_{1}, u_{3}, P\right) \mapsto(u, w, \bar{P})$, de modo a obter o espaço central como sendo o plano uw. Tal mudança é dada por

$$
\begin{aligned}
u_{1} & =\frac{\left(q_{021} b-q_{201} b-c q_{111}\right)}{b} u+ \\
& +\frac{1}{b\left(B k-B+3 q_{201} q_{111}\right)}\left(-2 q_{111} b q_{102}-Q_{211} k b+b Q_{211}-b q_{012} q_{201}+\right. \\
& \left.+2 q_{111}^{3}+q_{111} k C-q_{111} C-q_{111} k^{4}+q_{111} k^{3}+q_{201} q_{111} q_{021}\right) w \\
u_{3} & =w \\
P & =-\frac{\left(q_{021} b-q_{201} b-c q_{111}\right)\left(B k-B+3 q_{201} q_{111}\right)}{b^{2}(k-1)} u+ \\
& +\frac{\left(q_{021} b-q_{201} b-c q_{111}\right)\left(B k-B+3 q_{201} q_{111}\right)}{b^{3}(k-1)} w- \\
& -\frac{\left(q_{021} b-q_{201} b-c q_{111}\right)\left(B k-B+3 q_{201} q_{111}\right)}{b^{3}(k-1)} \bar{P}
\end{aligned}
$$


Substituindo (2.30) em $X_{\mathcal{L}}$ obtemos

$$
Y_{r}:=\left\{\begin{array}{rl}
\dot{u} & =w+f(u, w, \bar{P}) \\
\dot{w} & =g(u, w, \bar{P}) \\
\dot{\bar{P}} & =-b \bar{P}+h(u, w, \bar{P})
\end{array} .\right.
$$

onde $f(0,0,0)=g(0,0,0)=h(0,0,0)=0, \frac{\partial f}{\partial u}(0,0,0)=\frac{\partial f}{\partial w}(0,0,0)=\frac{\partial f}{\partial \bar{p}}(0,0,0)=$ $0, \frac{\partial g}{\partial u}(0,0,0)=\frac{\partial g}{\partial w}(0,0,0)=\frac{\partial g}{\partial \bar{p}}(0,0,0)=0, \frac{\partial h}{\partial u}(0,0,0)=\frac{\partial h}{\partial w}(0,0,0)=\frac{\partial h}{\partial \bar{p}}(0,0,0)=$ 0 .

A variedade central $W^{c}$, na origem, associada a $Y_{r}$ pode ser parametrizada por $\bar{P}=\bar{P}(u, w)$. A restrição de $Y_{r}$ à $W^{c}$ é dada por

$$
\left.Y_{r}\right|_{W^{c}}=\left\{\begin{array}{rl}
\dot{u} & =U(u, w) \\
\dot{w} & =W(u, w)
\end{array},\right.
$$

onde

$$
\begin{gathered}
\frac{\partial U}{\partial w}(0,0)=1, \\
\frac{\partial^{2} U}{\partial u^{2}}(0,0)=c B-\left(C-A+2 k^{3}\right) b+\frac{-2 q_{111} b+2 q_{201} b+2 q_{201} q_{111} c}{b(k-1)}=\chi \neq 0,
\end{gathered}
$$

$\mathrm{e}$

$$
\frac{\partial^{2} W}{\partial u w}(0,0)=\frac{\left(q_{021} b-q_{201} b-c q_{111}\right) q_{201} q_{111}}{b^{2}(k-1)} \neq 0
$$

Assim, o retrato de fase de $Y_{r}$ restrito a variedade central é como na figura 2.12.

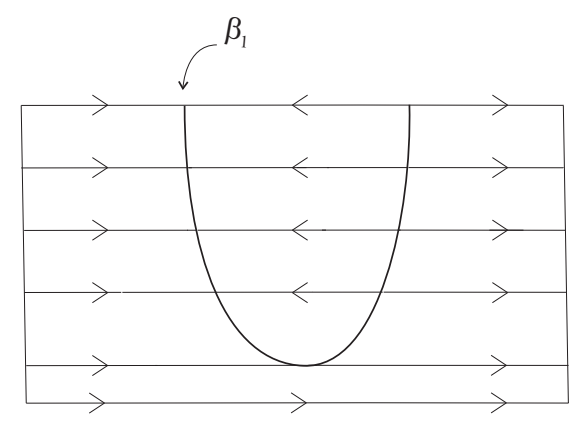

Figura 2.12: Retrato de fase de $\left.Y_{r}\right|_{W^{c}}$.

Disto, concluímos que existem variedades invariantes por $X_{\mathcal{L}}$,

$$
W_{\beta_{1}\left(u_{1}\right)}^{s} \text { e } W_{\beta_{1}(0)}^{c}
$$


cujo comportamento das curvas integrais, numa vizinhança de tais variedades, é como na figura 2.11.

Demonstração do teorema 2: Seja $p$ um ponto parcialmente umbílico do tipo $D_{23}$.

i) Lema $3 \Rightarrow p$ pertence a uma curva regular $c$ de pontos parcialmente umbílicos, escrita nas variáveis $u_{1}, u_{2}, u_{3}$ da forma

$$
c: u_{2}=c_{2}\left(u_{1}\right) \text { e } u_{3}=c_{3}\left(u_{1}\right) \text {. }
$$

ii) Lema $4 \Rightarrow$ o campo de Lie-Cartan, $X$, possui três curvas de equilíbrios $\beta_{i}, i=$ $1,2,3$, que no espaço das variáveis $u_{1}, u_{2}, u_{3}, P$ são da forma

$$
\beta_{i}: u_{2}=c_{2}\left(u_{1}\right), \quad u_{3}=c_{3}\left(u_{1}\right), \quad P=P_{i}\left(u_{1}\right), \quad i=1,2,3
$$

onde

$$
\begin{aligned}
& P_{1}(0)=0 \\
& P_{2}(0)=\frac{1}{2} \frac{c+\sqrt{c^{2}+4 b^{2}}}{b} \\
& P_{3}(0)=\frac{1}{2} \frac{c-\sqrt{c^{2}+4 b^{2}}}{b} .
\end{aligned}
$$

Observação $14 \Pi\left(\beta_{i}\right)=c, i=1,2,3$

Observação $15 P_{3}<P_{1}<P_{2}$, para $u_{1}$ suficientemente pequeno.

iii) Lema $5 \Rightarrow$ as curvas $\beta_{2}$ e $\beta_{3}$ são normalmente hiperbólicas do tipo sela para $X$ restrito à hipersuperfície de Lie-Cartan. Quanto a curva $\beta_{1}$, o retrato de fase de $X_{\mathcal{L}}$ é como na figura 2.11 .

Assim, conluímos que:

i), ii) e iii) $\Rightarrow$ a configuração principal, numa vizinhança de $p$, é como na figura 2.9. O que conclui o teorema 2. Veja figuras 2.13 e 2.14. 

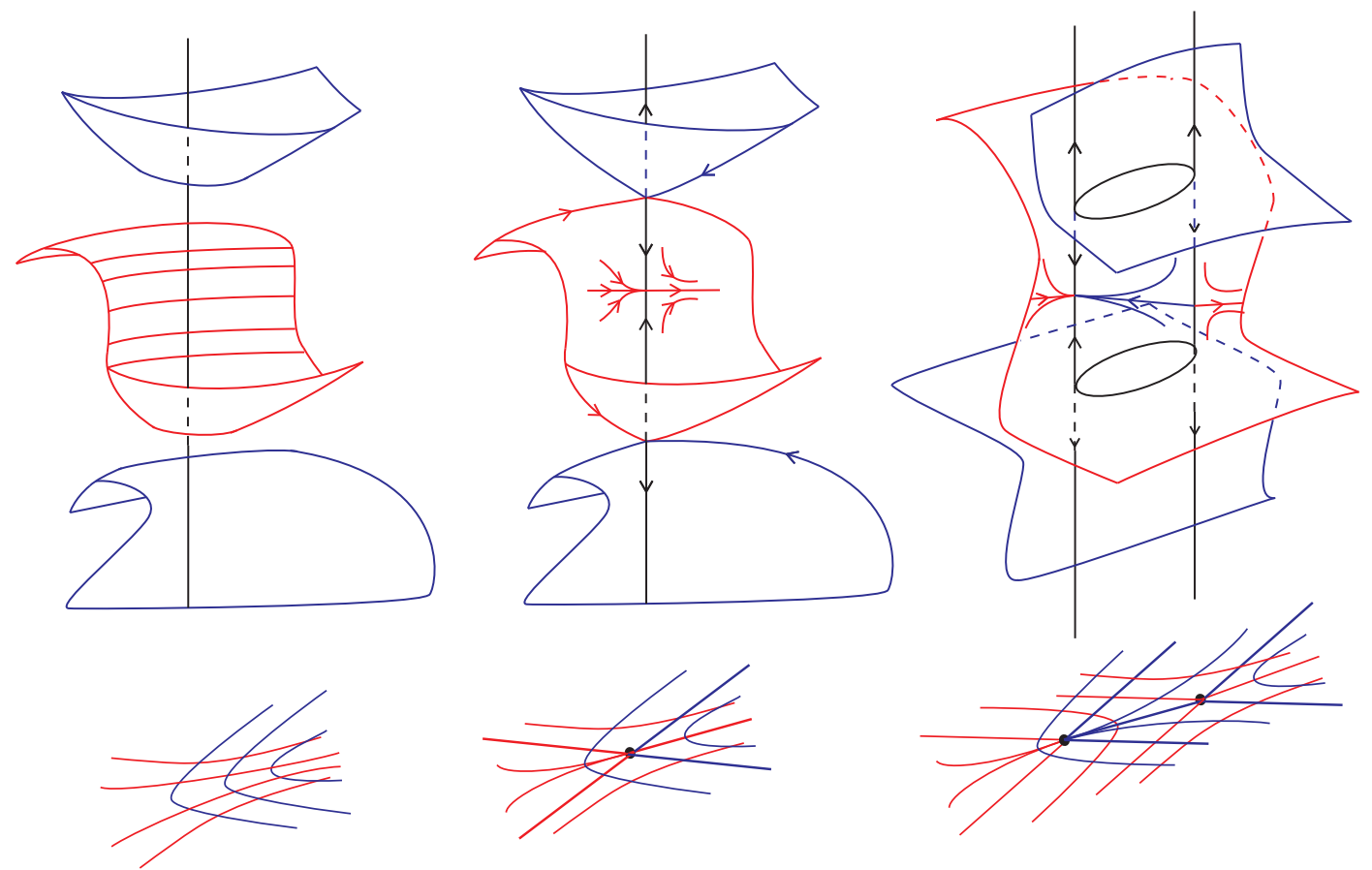

Figura 2.13: Seções da Hipersuperfície de Lie-Cartan e suas projeções na hipersuperfície imersa em $\mathbb{R}^{4}$. 

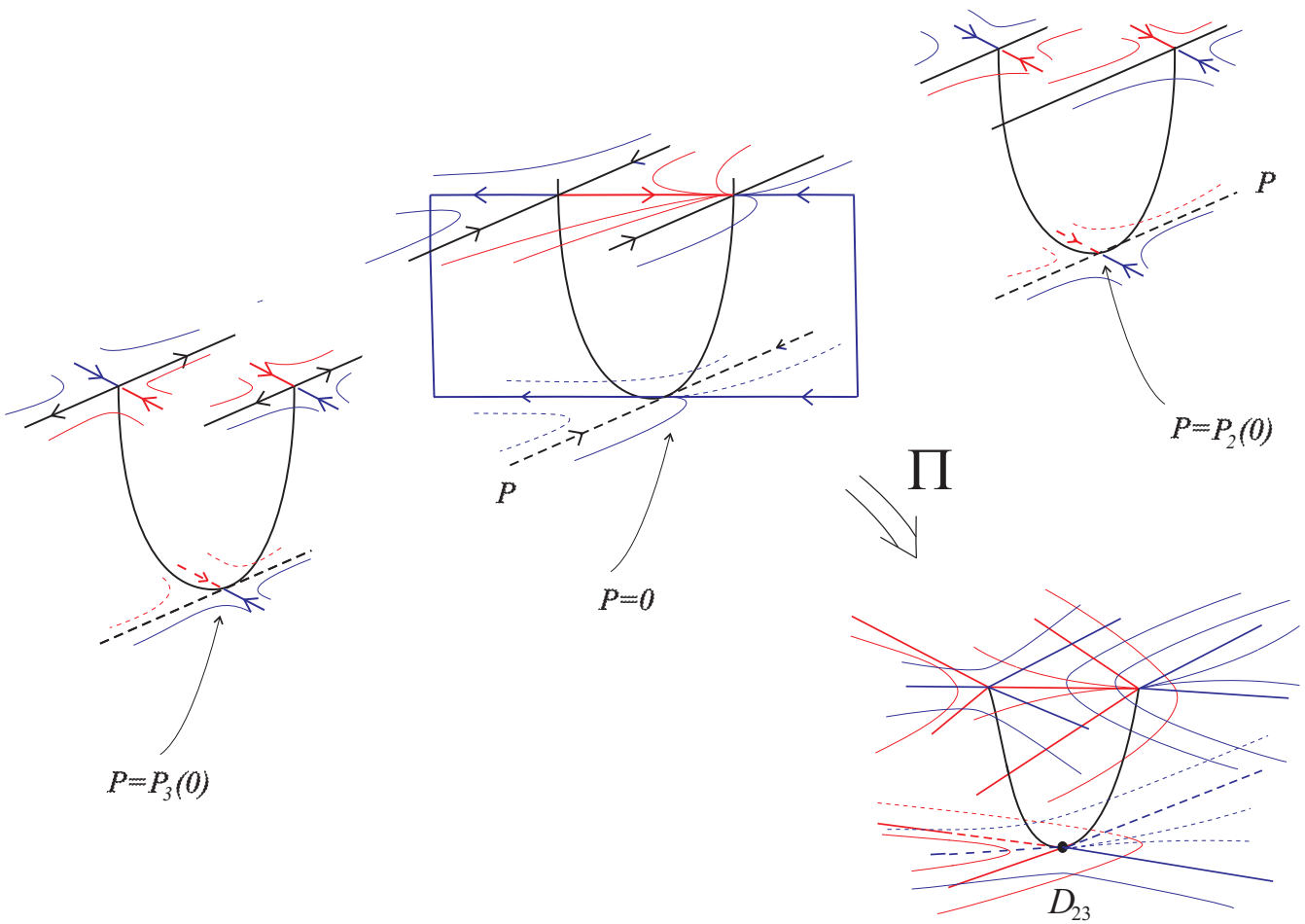

Figura 2.14: Projeção das curvas integrais de $X_{\mathcal{L}}$ na hipersuperfície imersa em $\mathbb{R}^{4}$. Observamos que, numa vizinhança de $P_{2}(0)=\frac{c+\sqrt{c^{2}+4 b^{2}}}{2 b}$ e de $P_{3}(0)=\frac{c-\sqrt{c^{2}+4 b^{2}}}{2 b}$, podemos escrever a hipersuperfície de Lie-Cartan da forma $u_{3}=u_{3}\left(u_{1}, u_{2}, P\right.$ ) (veja a equação (2.27)) e, numa vizinhaça de $P_{1}(0)=0$, a hipersuperfície de Lie-Cartan é dada por $u_{2}=u_{2}\left(u_{1}, u_{3}, P\right)$ (veja a equação $\left.(2.42)\right)$.

Na figura 2.15 descrevemos as variedades invariantes estratificadas. 


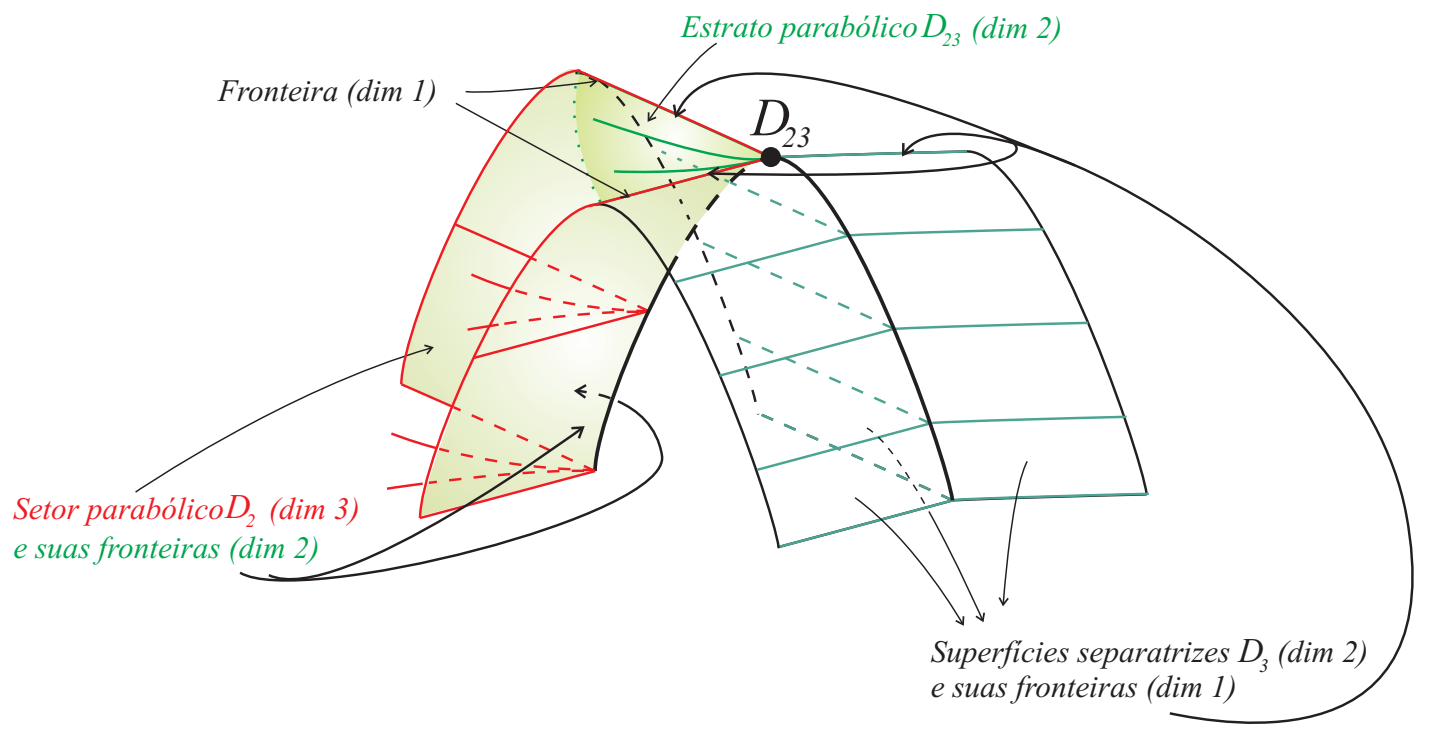

Figura 2.15: Variedades invariantes estratificadas numa vizinhança de um ponto do tipo $D_{23}$.

\subsection{Linhas de curvatura em torno de um ponto do tipo $D_{12}$}

Nesta seção, provaremos o seguinte teorema:

Teorema 3 (R. Garcia, [6]) Suponhamos $\alpha \in \mathcal{I}^{k}\left(\mathbb{M}^{3}, \mathbb{R}^{4}\right), k \geq 4$, e p um ponto parcialmente umbílico do tipo $D_{12}$. Então o comportamento das linhas de curvatura próximas à p é como na figura 2.16. 


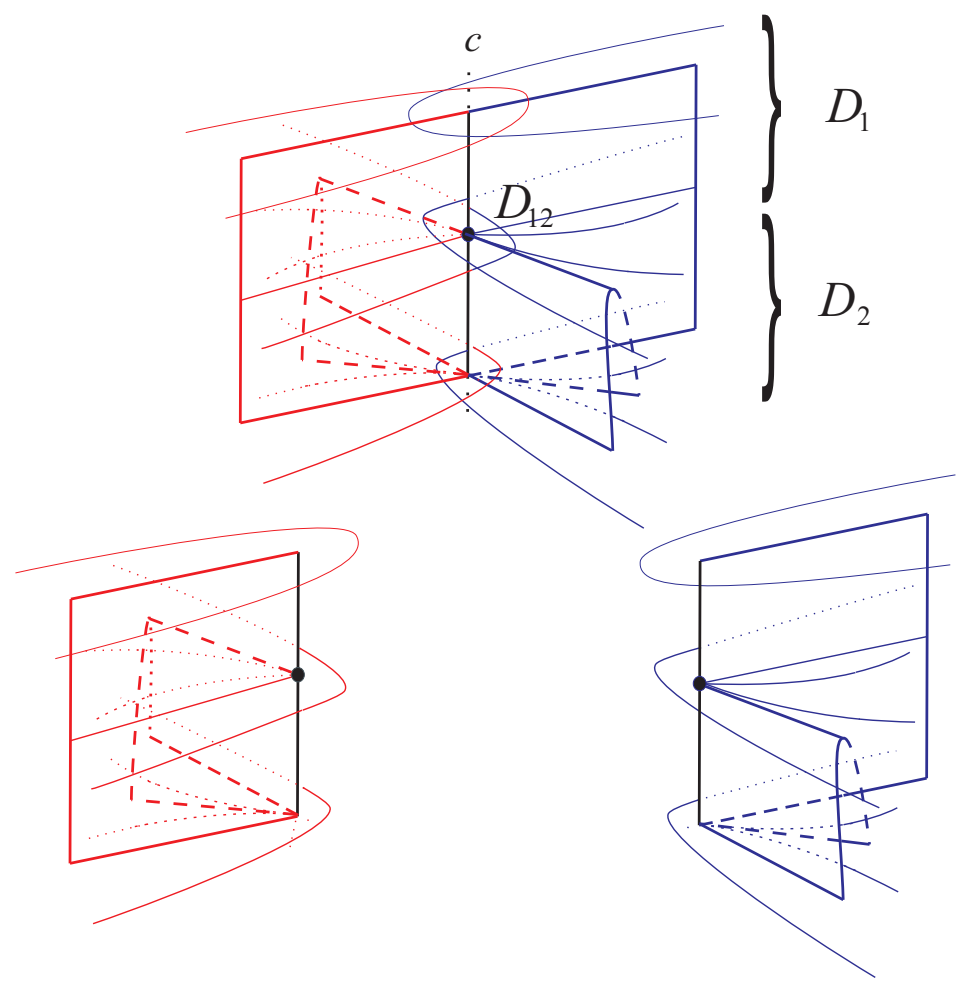

Figura 2.16: Configuração Principal numa vizinhança de um ponto parcialmente umbílico do tipo $D_{12}$.

No capítulo 1, vimos que na carta de Monge reduzida as condições para que $p$ seja do tipo $D_{12}$ são:

1) $c b(b-a) \neq 0$ e $\frac{a}{b}=2$,

2) $\chi_{2} \neq 0$, onde

$$
\begin{aligned}
\chi_{2} & =\frac{1}{27 b^{6}(k-1)}\left(b q_{111} k^{4}-b q_{111} k^{3}-k B b q_{201}-k B q_{111} c+Q_{211} k b^{2}\right. \\
& -b q_{111} k C+k B b q_{021}-Q_{211} b^{2}-B b q_{021}+b q_{111} C+B b q_{201} \\
& -3 q_{111}^{2} q_{201} c-3 q_{111} q_{201}^{2} b+2 q_{111} b^{2} q_{102}+B q_{111} c-2 b q_{111}{ }^{3} \\
& \left.+2 q_{111} q_{201} b q_{021}+b^{2} q_{012} q_{201}\right) c^{3} .
\end{aligned}
$$

Usando a condição 1) mostraremos, no lema 6 , que um ponto do tipo $D_{12}$ pertence a uma curva regular de pontos parcialmente umbílicos. 
Já a condição 2), garante a transição $D_{1}-D_{2}$ ao longo da curva parcialmente umbílica, veja lema 7 mais adiante.

Lema 6 Se p é um ponto parcialmente umbílico Semi-Darbouxiano do tipo $D_{12}$, então $p$ pertente a uma curva regular c de pontos parcialmente umbílicos. Além disso, na carta de Monge reduzida com $k_{3}=1$, escrevemos

$$
c: \begin{aligned}
& u_{1}=c_{1}\left(u_{3}\right)=\frac{b q_{021}-q_{201} b-q_{111} c}{b^{2}} u_{3}+O(2), \\
& u_{2}=c_{2}\left(u_{3}\right)=-\frac{q_{111}}{b} u_{3}+O(2),
\end{aligned}
$$

numa vizinhança de $p$.

Demonstração: Vimos no capítulo 1 (observação 5) que os pontos parcialmente umbílicos são dados por

$$
L_{r}\left(u_{1}, u_{2}, u_{3}\right)=0 \text { e } M_{r}\left(u_{1}, u_{2}, u_{3}\right)=0 .
$$

Como, na carta de Monge reduzida com $k_{3}=1$,

$$
\begin{aligned}
& L_{r}=-b v-q_{111} w+O(2), \\
& M_{r}=-b u+c v-\left(q_{201}-q_{021}\right) w+O(2), \\
& N_{r}=b v+q_{111} w+O(2) .
\end{aligned}
$$

segue-se que

$$
\left.\frac{\partial\left(L_{r}, M_{r}\right)}{\partial\left(u_{1}, u_{2}, u_{3}\right)}\right|_{u_{1}=0, u_{2}=0, u_{3}=0}=\left(\begin{array}{ccc}
0 & -b & -q_{111} \\
-b & c & -q_{201}+q_{021}
\end{array}\right),
$$

donde

$$
\left.\operatorname{det}\left(\frac{\partial\left(L_{r}, M_{r}\right)}{\partial\left(u_{1}, u_{2}\right)}\right)\right|_{u_{1}=0=u_{2}=u_{3}}=-b^{2} .
$$

Assim, pelo teorema da função implícita, teremos que $p$ pertence a uma curva regular de pontos parcialmente umbílicos. Além disso, podemos escrever $u_{1}=c_{1}\left(u_{3}\right)$ e $u_{2}=c_{2}\left(u_{3}\right)$ em (2.33). Fazendo

$$
\begin{aligned}
& c_{1}\left(u_{3}\right)=c_{1}^{1} u_{3} \\
& c_{2}\left(u_{3}\right)=c_{3}^{1} u_{3}
\end{aligned}
$$

e resolvendo

$$
\left\{\begin{array}{c}
\frac{\partial}{\partial u_{1}}\left[L_{r}\left(c_{1}\left(u_{3}\right), c_{2}\left(u_{3}\right), u_{3}\right)\right]=0 \\
\frac{\partial}{\partial u_{1}}\left[M_{r}\left(c_{1}\left(u_{3}\right), c_{2}\left(u_{3}\right), u_{3}\right)\right]=0
\end{array}\right.
$$


em $c_{2}^{1}$ e $c_{3}^{1}$, obtemos que

$$
\begin{aligned}
& c_{1}\left(u_{3}\right)=\frac{b q_{021}-q_{201} b-q_{111} c}{b^{2}} u_{3}+O(2) \\
& c_{2}\left(u_{3}\right)=-\frac{q_{111}}{b} u_{3}+O(2)
\end{aligned}
$$

Lema 7 Sejam $\left(u_{1}, u_{2}, u_{3}\right): \mathbb{M}^{3} \rightarrow \mathbb{R}^{3}$ a carta de Monge reduzida com $k_{3}=1, X$ o campo de Lie-Cartan dado em (2.2) e $c\left(u_{3}\right)=\left(c_{1}\left(u_{3}\right), c_{2}\left(u_{3}\right), u_{3}\right)$ uma curva parcialmente umbilica tal que $p=c(0)$ é do tipo $D_{12}$. Para $u_{3}$ suficientemente pequeno, o conjunto dos equilíbrios de $X$, que se projetam em c, é formado por duas curvas regulares, que denotaremos por $\beta_{1}$ e $\beta_{2}$, onde $\beta_{1}$ intersecta o eixo $P$ transversalmente em $P=c / b$, e $\beta_{2}$ passa pela origem e é tangente ao eixo $P$ neste ponto.

Demonstração: Sendo

$$
X=\left\{\begin{array}{l}
\dot{u}_{1}=\mathcal{L}_{P} \\
\dot{u}_{2}=P \mathcal{L}_{P} \\
\dot{u}_{3}=(\mathcal{U}+\mathcal{V} P) \mathcal{L}_{P} \\
\dot{P}=-\left(\mathcal{L}_{u_{1}}+P \mathcal{L}_{u_{2}}+\mathcal{L}_{u_{3}}(\mathcal{U}+\mathcal{V} P)\right)
\end{array}\right.
$$

segue-se que os equilíbrios de $X$ são dados por

$$
\left\{\begin{array}{l}
\mathcal{L}_{P}\left(u_{1}, u_{2}, u_{3}\right)=0 \\
\left(\mathcal{L}_{u_{1}}+P \mathcal{L}_{u_{2}}+\mathcal{L}_{u_{3}}(\mathcal{U}+\mathcal{V} P)\right)\left(u_{1}, u_{2}, u_{3}, P\right)=0
\end{array}\right.
$$

Como

$$
\mathcal{L}_{P}\left(u_{1}, u_{2}, u_{3}\right)=2 P L_{r}\left(u_{1}, u_{2}, u_{3}\right)+M_{r}\left(u_{1}, u_{2}, u_{3}\right),
$$

resulta que os pontos parcialmente umbílicos, que neste caso formam uma curva regular $c$, anulam a primeira equação de (2.35). Pelo lema 6 podemos escrever as coordenadas de $c$ da forma

$$
u_{1}=c_{1}\left(u_{3}\right), \quad u_{2}=c_{2}\left(u_{3}\right),
$$

e, além disso,

$$
\begin{aligned}
& c_{1}\left(u_{3}\right)=\frac{b q_{021}-q_{201} b-q_{111} c}{b^{2}} u_{3}+O(2), \\
& c_{2}\left(u_{3}\right)=-\frac{q_{111}}{b} u_{3}+O(2),
\end{aligned}
$$


Seja

$$
X_{4}\left(u_{3}, P\right)=\left(\mathcal{L}_{u_{1}}+P \mathcal{L}_{u_{2}}+\mathcal{L}_{u_{3}}(\mathcal{U}+\mathcal{V} P)\right)\left(c_{1}\left(u_{3}\right), c_{2}\left(u_{3}\right), u_{3}, P\right) .
$$

Substituindo (2.37) na última equação de (2.3) e fazendo $a=2 b$, obtemos que

$$
\left.\frac{\partial X_{4}\left(u_{3}, P\right)}{\partial P}\right|_{u_{3}=0, P=\frac{c}{b}} \neq 0
$$

e

$$
\left.\frac{\partial X_{4}\left(u_{3}, P\right)}{\partial P}\right|_{u_{3}=0, P=0}=0,\left.\quad \frac{\partial^{2} X_{4}\left(u_{3}, P\right)}{\partial P^{2}}\right|_{u_{3}=0, P=0}=-c \neq 0
$$

Assim,

$(2.38) \Rightarrow$ existe uma única curva de equilíbrios, $\beta_{1}$, transversal ao eixo $P$, passando por $u_{3}=0, P=c / b$,

$(2.39) \Rightarrow$ existe uma única curva, $\beta_{2}$, passando pela origem e tangente ao eixo $P$ neste ponto.

Como ilustração veja a figura (2.17).

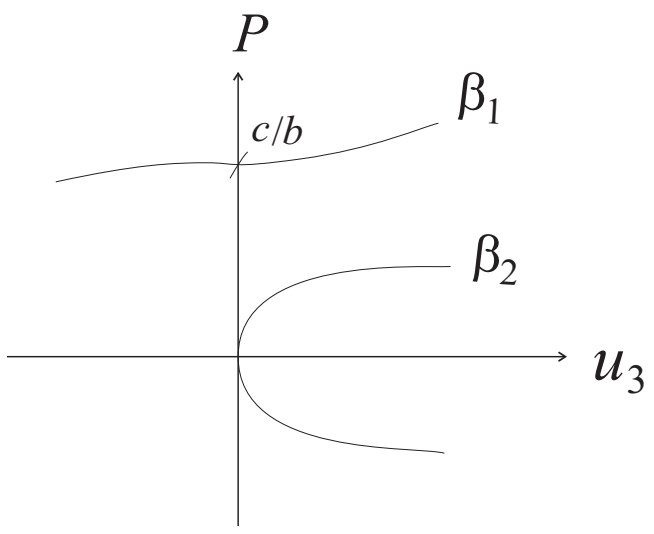

Figura 2.17: Curvas $\beta_{1}$ e $\beta_{2}$ no plano $u_{3} P$. 
Observação 16 Vimos, em (2.3) que $X_{4}$ é cúbica em P. Substituindo (2.34) em (2.3) e fazendo $a=2 b$ teremos que, para um ponto do tipo $D_{12}$,

$$
X_{4}\left(c_{1}\left(u_{3}\right), c_{2}\left(u_{3}\right), u_{3}, P\right)=A_{3}\left(u_{3}\right) P^{3}+A_{2}\left(u_{3}\right) P^{2}+A_{1}\left(u_{3}\right) P+A_{0}\left(u_{3}\right),
$$

onde $A_{3}(0)=b, A_{2}(0)=-c, A_{1}(0)=0$ e $A_{0}(0)=0$. $O$ discriminante de

$$
A_{3}\left(u_{3}\right) P^{3}+A_{2}\left(u_{3}\right) P^{2}+A_{1}\left(u_{3}\right) P+A_{0}\left(u_{3}\right)=0
$$

é

$$
D\left(u_{3}\right)=\chi_{2} u_{3}+O(2)
$$

onde $\chi_{2}$ foi dada em (2.32). Assim, supondo $\chi_{2}<0$ (o caso $\chi_{2}>0$ é análogo) teremos:

- Para $u_{3}<0$, (2.40) possui uma única raíz real;

- Para $u_{3}=0$, (2.40) possui uma raíz real dupla em $P=0$ e uma simples em $P=\frac{c}{b}$

- Para $u_{3}>0$, (2.40) possui três raízes reais.

Para que tenhamos a configuração na figura 2.16, devemos mostrar que $\beta_{1}$ é normalmente hiperbólica do tipo sela, e que $\beta_{2}$ é do tipo sela nó (veja ilustração na figura 2.18):

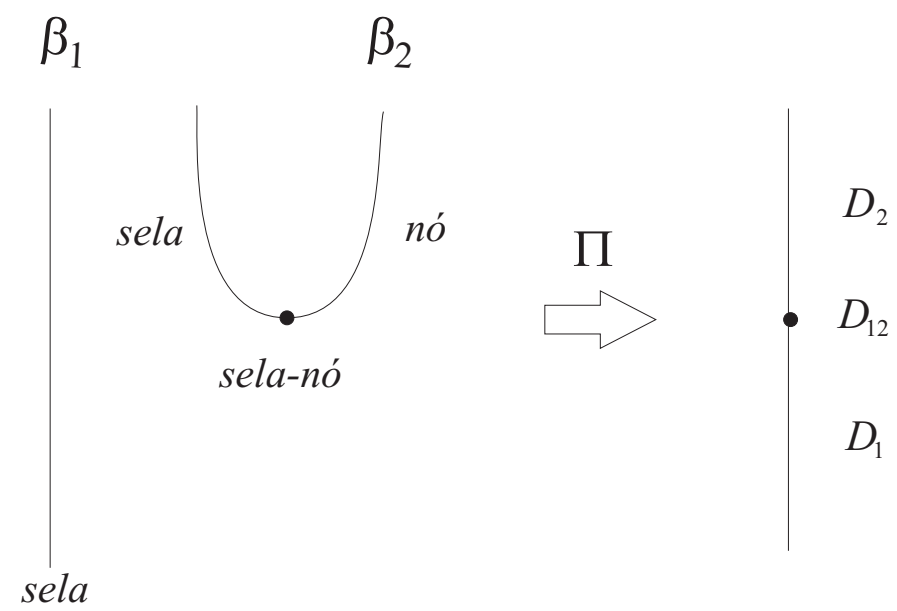

Figura 2.18: Equilíbrios de $X$ associados a um ponto parcialmente umbílico do tipo $D_{12}$. 
Lema 8 Seja $X$ o campo de Lie-Cartan e $\beta_{1}, \beta_{2}$ as respectivas curvas de equilíbrios encontradas no lema \%. Então,

- $\beta_{1}$ é normalmente hiperbólica do tipo sela para $X$ restrito à hipersuperfície de Lie-Cartan;

- Quanto a curva $\beta_{2}$, o retrato de fase de X restrito à hipersuperfície de Lie-Cartan, numa vizinhança da origem, é como na figura 2.19.

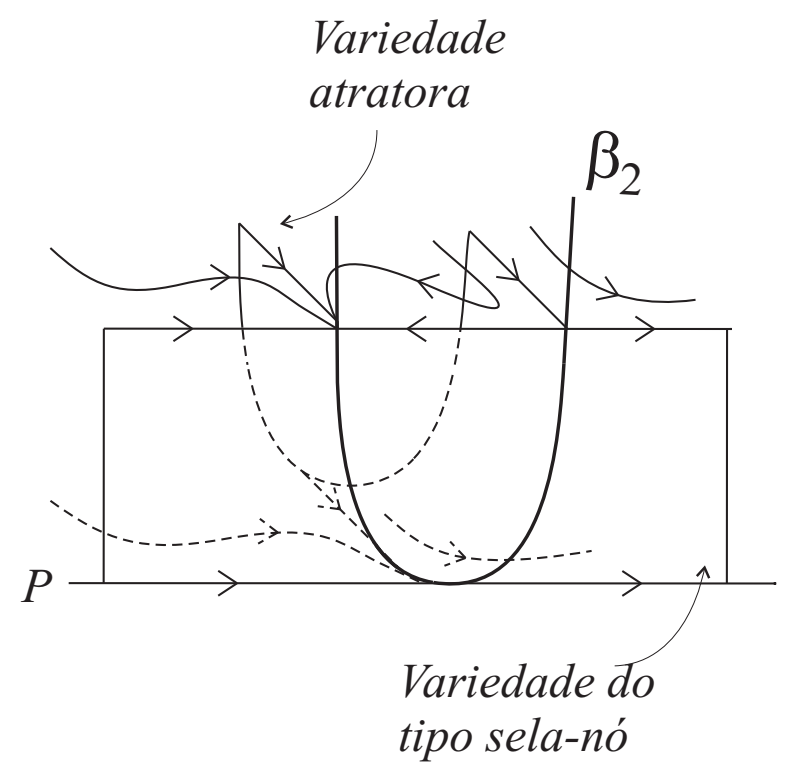

Figura 2.19: Retrato de fase de $X$, restrito à hipersuperfície de Lie-Cartan, numa vizinhança da origem. 
Demonstração: Para $u_{3}$ suficientemente pequeno, os autovalores não nulos da linearização $D X\left(\beta_{1}\left(u_{3}\right)\right)$ do campo de Lie-Cartan são:

$$
\lambda_{1}\left(u_{3}\right)=-\frac{b^{2}+c^{2}}{b}+O(1), \quad \lambda_{2}\left(u_{3}\right)=\frac{c^{2}}{b}+O(1) .
$$

Disto, restrito à hipersuperfície de Lie-Cartan, $\beta_{1}$ é normalmente hiperbólica do tipo sela.

Passemos ao estudo do campo de Lie-Cartan restrito a hipersuperfície de LieCartan numa vizinhança de $\beta_{2}$.

Seja $\mathcal{L}\left(u_{1}, u_{2}, u_{3}, P\right)=0$ a equação que define a hipersuperfície de Lie-Cartan, onde

$$
\mathcal{L}\left(u_{1}, u_{2}, u_{3}, P\right)=L_{r}\left(u_{1}, u_{2}, u_{3}\right) P^{2}+M_{r}\left(u_{1}, u_{2}, u_{3}\right) P+N_{r}\left(u_{1}, u_{2}, u_{3}\right)
$$

Na Carta de Monge reduzida, temos que

$$
\begin{aligned}
& L_{r}=-b u_{2}-q_{111} u_{3}+O(2), \\
& M_{r}=-b u_{1}+c u_{2}-\left(q_{201}-q_{021}\right) u_{3}+O(2), \\
& N_{r}=b u_{2}+q_{111} u_{3}+O(2) .
\end{aligned}
$$

Assim,

$$
\frac{\partial \mathcal{L}}{\partial u_{2}}(0,0,0,0)=b \neq 0
$$

implicando, pelo teorema da função implícita, que podemos escrever $u_{2}=u_{2}\left(u_{1}, u_{3}, P\right)$ na equação $\mathcal{L}\left(u_{1}, u_{2}, u_{3}, P\right)=0$, numa vizinhança da origem. A expansão em Taylor, até segunda ordem, de $u_{2}\left(u_{1}, u_{3}, P\right)$ é a seguinte

$$
\begin{aligned}
u_{2}\left(u_{1}, u_{3}, P\right) & =-\frac{q_{111}}{b} u_{3}-\frac{1}{2 b}\left(B+2 \frac{q_{201} q_{111}}{k-1}\right) u_{1}^{2}+\left(\frac{q_{201}}{b}-\frac{q_{021}}{b}+\frac{c q_{111}}{b^{2}}\right) u_{3} P \\
& +\left(\frac{q_{201} q_{111} q_{021}}{(k-1) b^{2}}-\frac{q_{201} q_{012}}{(k-1) b}+\frac{q_{111}{ }^{3}}{(k-1) b^{2}}+\frac{q_{111} C}{b^{2}}-\frac{q_{102} q_{111}}{(k-1) b}\right. \\
& \left.-\frac{q_{111} k^{3}}{b^{2}}-\frac{Q_{211}}{b}\right) u_{1} u_{3}+\left(-\frac{q_{111}{ }^{3} q_{021}}{(k-1) b^{3}}+\frac{q_{111} q_{021} q_{102}}{(k-1) b^{2}}-\frac{1}{2} \frac{D q_{111}{ }^{2}}{b^{3}}\right. \\
& \left.+\frac{q_{111}^{2} q_{012}}{(k-1) b^{2}}+\frac{Q_{121} q_{111}}{b^{2}}-\frac{1}{2} \frac{Q_{112}}{b}-\frac{q_{012} q_{102}}{b(k-1)}\right) u_{3}^{2}+O(3)
\end{aligned}
$$

Substituindo $u_{2}=u_{2}\left(u_{1}, u_{3}, P\right)$ nas coordenadas de $X$ dadas em (2.3) com $a=2 b$, obtemos a restrição do campo de Lie-Cartan à hipersuperfície de Lie-Cartan:

$$
X_{\mathcal{L}}=\left\{\begin{array}{l}
\left.\dot{u}_{1}=X_{1}\left(u_{1}, u_{2}\left(u_{1}, u_{3}, P\right), u_{3}, P\right)\right) \\
\dot{u}_{3}=X_{3}\left(u_{1}, u_{2}\left(u_{1}, u_{3}, P\right), u_{3}, P\right) \\
\dot{p}=X_{4}\left(u_{1}, u_{2}\left(u_{1}, u_{3}, P\right), u_{3}, P\right)
\end{array}\right.
$$


onde

$$
\begin{aligned}
X_{1} & =-b u_{1}+\frac{\left(q_{021} b-c q_{111}-q_{201} b\right)}{b} u_{3}+O(2) \\
X_{3} & =\left(\mathcal{U}\left(u_{1}, u_{2}\left(u_{1}, u_{3}, P\right), u_{3}\right)+P \mathcal{V}\left(u_{1}, u_{2}\left(u_{1}, u_{3}, P\right), u_{3}\right)\right) X_{1} \\
X_{4} & =\left(-B-3 \frac{q_{201} q_{111}}{k-1}\right) u_{1}+ \\
& +\left(-Q_{211}+\frac{q_{111} C}{b}+\frac{k^{3}}{b}-\frac{2 q_{102} q_{111}}{k-1}-\frac{q_{201} q_{012}}{k-1}+\frac{2 u_{3} q_{111}{ }^{3}}{(k-1) b}+\frac{q_{201} q_{021} q_{111}}{(k-1) b}\right) u_{3}- \\
& +O(2)
\end{aligned}
$$

A linearização $D X_{\mathcal{L}}\left(\beta_{2}\left(u_{3}\right)\right)$ possui um autovalor nulo (associado à direção tangente a curva de equilíbrios) e os seguintes dois, escritos em função de P:

$$
\lambda_{1}(P)=3 b P^{2}-2 c P+O(3) \text { e } \lambda_{2}(P)=-b+c P-2 b P^{2}+O(3) .
$$

Sem perda de generalidade, podemos supor $b, c>0$.

Sendo $\lambda_{2}\left(u_{3}\right)<0$, para $u_{3}$ suficientemente pequeno, segue-se do teorema 4 , no apêndice deste capítulo, que existe uma variedade, denotada por $W^{s}\left(\beta_{2}\left(u_{3}\right)\right)$, invariante por $X_{\mathcal{L}}$, de classe $C^{k-2}$ e onde $\beta_{2}\left(u_{3}\right)$ é atratora.

Seja $\varepsilon>0$ suficientemente pequeno. Definamos

$$
W=\left\{\left(u_{1}, u_{2}, u_{3}, P\right), \quad\left|u_{3}\right|<\varepsilon, \quad u_{1}=c_{1}\left(u_{3}\right) \text { e } u_{2}=c_{2}\left(u_{3}\right)\right\} .
$$

Então,

1. $W$ é uma variedade diferenciável de classe $C^{k-2}$ e invariante pelo fluxo de $X$.

2. $W$ está contida na hipersuperfície de Lie-Cartan

3. As coordendas de um ponto de $W$ satisfaz:

$$
\dot{u}_{1}=0, \dot{u}_{2}=0, \dot{u}_{3}=0
$$

e

$$
\dot{P}=A_{3}\left(u_{3}\right) P^{3}+A_{2}\left(u_{3}\right) P^{2}+A_{1}\left(u_{3}\right) P+A_{0}\left(u_{3}\right)
$$

onde $A_{3}(0)=b, A_{2}(0)=-c, A_{1}(0)=A_{0}(0)=0$.

Nas variáveis $u_{3}$ e $P$ vemos, de (2.39), que a solução de $\dot{P}=0$ é da forma

$$
u_{3}(P)=-c P^{2}+O(3) \text {. }
$$

Assim 
- $u_{1}=0=u_{2}=u_{3} \Rightarrow \dot{P}=-c P^{2}+b P^{3}<0$ para $P<\frac{c}{b}$,

- $u_{1}=c_{1}\left(u_{3}\right)$ e $u_{2}=c_{2}\left(u_{3}\right) \Rightarrow\left\{\begin{array}{l}\dot{P}>0 \text { se }|P|<u_{3}(P) \\ \dot{P}<0 \text { se }|P|>u_{3}(P)\end{array}\right.$

Veja figura 2.20.

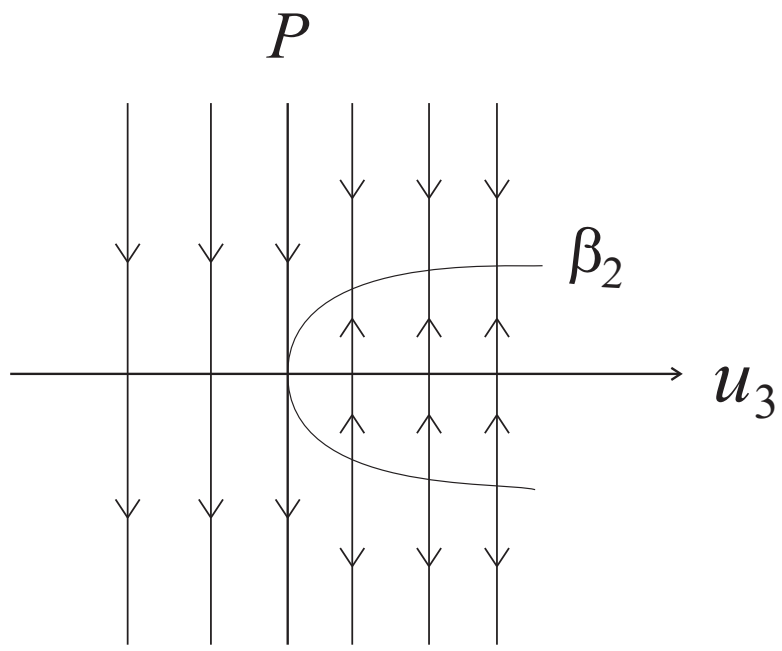

Figura 2.20: Retrato de fase de $X$ restrito a $W$ no ponto $\beta_{2}(0)$.

Prova do teorema 3: Seja $p$ um ponto parcialmente umbílico semi-Darbouxiano do tipo $D_{12}$. Então

1) Lema $6 \Rightarrow p$ pertence a uma curva regular de pontos parcialmente umbílicos.

2) Lema $7 \Rightarrow$ o campo de Lie-Cartan, restrito a hipersuperfície de Lie-Cartan, possui duas curvas de equilíbrios $\beta_{1}$ e $\beta_{2}$ que se projetam em $c$.

3) Lema $8 \Rightarrow \beta_{1}$ é normalmente hiperbólica do tipo sela, e o retrato de fase do campo de Lie-Cartan restrito a hipersuperfície de Lie Cartan, numa vizinhança de $\beta_{2}$, é como na figura 2.19.

Usando o levantamento de Lie-Cartan, vemos que 1), 2) e 3) implicam que o comportamento das linhas de curvatura numa vizinhança de um ponto parcialmente umbílico do tipo $D_{12}$ é como na figura 2.16. Como ilustração, veja figura 2.21. 

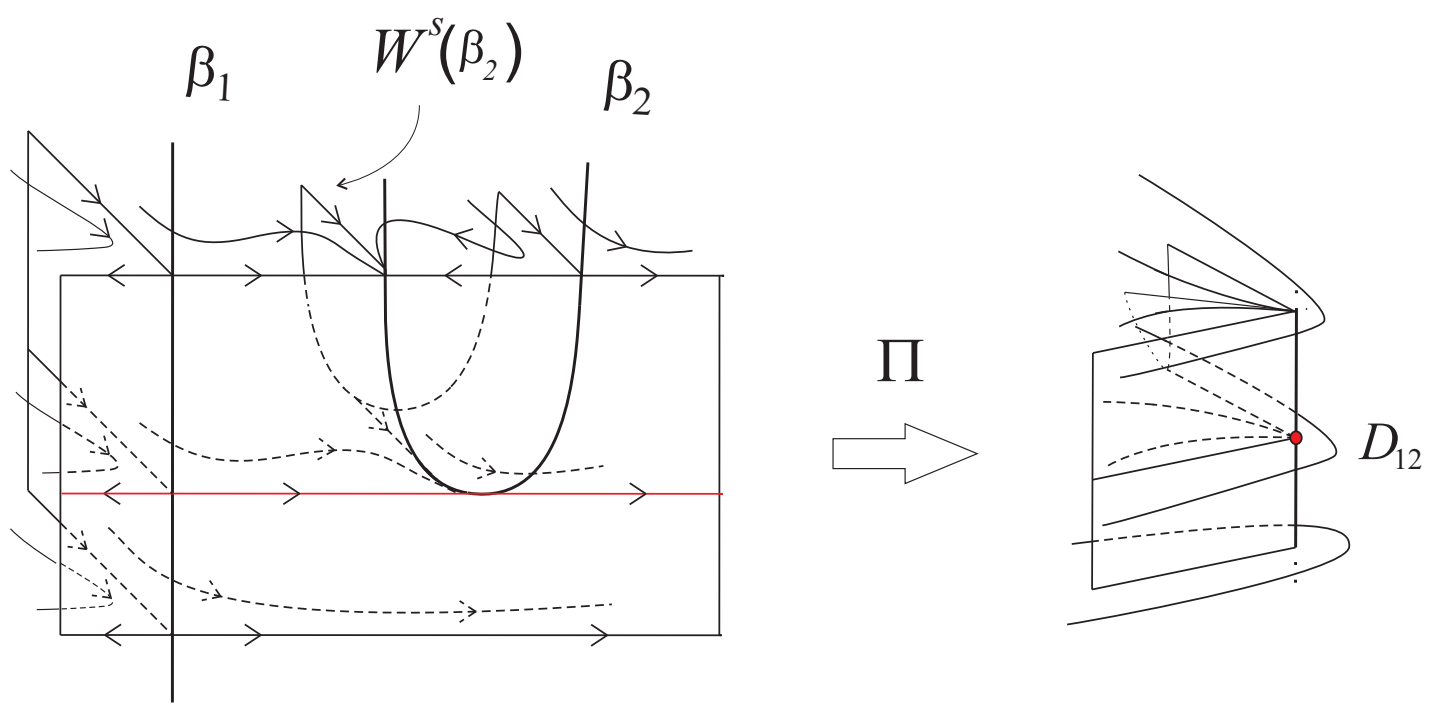

Figura 2.21: Resolução de Lie-Cartan para um ponto parcialmente umbílico do tipo $D_{12}$.

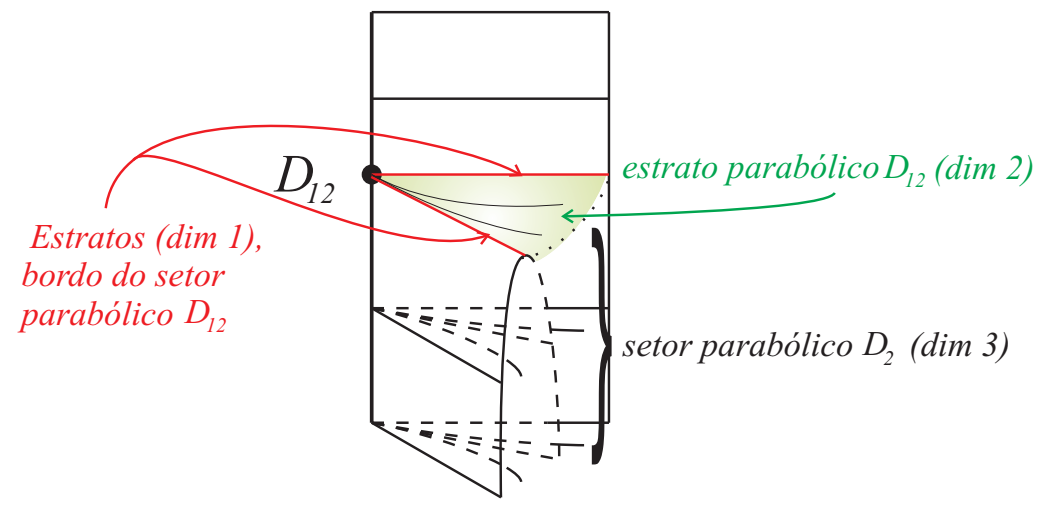

Figura 2.22: Variedades invariantes estratificadas numa vizinhança de um ponto do tipo $D_{12}$.

\subsection{Apêndice}

O seguinte resumo é baseado no apêndice de [6], o reescrevemos aqui para facilitar a leitura.

Sejam $M$ uma variedade $m$ dimensional e de classe $C^{r+1}, X: M \rightarrow T M$ um campo de vetores de classe $C^{r}$ e $\mathcal{E} \subset M$ uma subvariedade de classe $C^{r}$ e de codimensão $l>0$ tal que $\left.X\right|_{\mathcal{E}} \equiv 0$. 
Sejam $D X(p): T_{p} M \rightarrow T_{p} M$ a linearização de $X$ em $p \in \mathcal{E}$ e $Q X(p)$ a aplicação no espaço quociente $T_{p} M / T_{p} \mathcal{E}$ para $p \in \mathcal{E}$.

Para cada $p \in \mathcal{E}$, sejam $E_{p}^{s}, E_{p}^{c}$ e $E_{p}^{u}$ os autoespaços invariantes de $T_{p} M$ associados aos autovalores de $D X(p)$ que têm parte real negativa, parte real nula e parte real positiva.

Dizemos que $\mathcal{E}$ é um conjunto normalmente hiperbólico para $X$ se $Q X(p)$ for invertível para todo $p \in \mathcal{E}$. Se ambos os autoespaços $E_{p}^{s}$ e $E_{p}^{u}$ forem não vazios, diremos que $\mathcal{E}$ é normalmente hiperbólico do tipo sela. Se um deles for vazio e o outro não, diremos que $\mathcal{E}$ é normalmente hiperbólico do tipo repulsor $\left(E_{p}^{s}=\emptyset\right)$, ou normalmente hiperbólico do tipo atrator $\left(E_{p}^{u}=\emptyset\right)$.

Supondo $\mathcal{E}$ normalmente hiperbólico, teremos que $E_{p}^{c}=T_{p} \mathcal{E}$.

Dizemos que uma variedade $W^{s}(\mathcal{E})$ é uma variedade estável para $X$ se $\mathcal{E} \subset W^{s}(\mathcal{E})$, $W^{s}(\mathcal{E})$ for localmente invariante pelo fluxo de $X$ e $W^{s}(\mathcal{E})$ for tangente à $E_{p}^{s} \oplus E_{p}^{c}$ em $p$ para todo $p \in \mathcal{E}$. Definimos $W^{u}(\mathcal{E})$ de maneira análoga.

Teorema 4 Seja $M$ uma variedade de classe $C^{r+1}, 1 \leq r \leq \infty$. Seja $X: M \rightarrow T M$ um campo vetorial de classe $C^{r}$ e seja $\mathcal{E} \subset M$ um conjunto normalmente hiperbólico para $X$. Sejam $k_{1}$ e $k_{2}$ inteiros fixados, tais que $Q X(p)$ possui $k_{1}$ autovalores com parte real positiva e $k_{2}$ autovalores com parte real negativa para todo $p \in \mathcal{E}$. Então:

i) existência: existem variedades estável $W^{s}(\mathcal{E})$ e instável $W^{u}(\mathcal{E})$ tangentes em $p \in \mathcal{E} \grave{a} E_{p}^{s} \oplus E_{p}^{c}$ e $E_{p}^{u} \oplus E_{p}^{c}$, respectivamente.

ii) diferenciabilidade: $W^{u}(\mathcal{E})$ e $W^{s}(\mathcal{E})$ são de classe $C^{r}, 1 \leq r \leq \infty$.

iii) unicidade: qualquer conjunto localmente invariante contido numa vizinhança de $\mathcal{E}$ está contido em $W^{u}(\mathcal{E}) \cup W^{s}(\mathcal{E})$.

iv) invariância: Existe uma vizinhança $U$ de $\mathcal{E}$ tal que $\left\{p \in U: X_{t}(p) \in U, \forall t \in\right.$ $(-\infty, \infty)\}=\mathcal{E},\left\{p \in U: X_{t}(p) \in U, \forall t \geq 0\right\} \subset W^{s}(\mathcal{E})$ e $\left\{p \in U: X_{t}(p) \in\right.$ $U, \forall t \leq 0\} \subset W^{u}(\mathcal{E})$, onde $X_{t}(p)$ é o fluxo de $X$

v) caracterização: $W^{s}(\mathcal{E})=\{p \in M: \omega(p) \subset \mathcal{E}\}$ e $W^{u}(\mathcal{E})=\{p \in M: \alpha(p) \subset \mathcal{E}\}$, onde $\omega(p)$ denota o $\omega$-limite de $p$ e $\alpha(p)$ denota o $\alpha$-limite de $p$.

vi) laminação: $W^{s}(\mathcal{E})$ é fibrada por uma família de subvariedades de classe $C^{r}$ $\left\{W^{s s}(p): p \in \mathcal{E}\right\}$ localmente invariantes e tais que $T_{p} W^{s s}(\mathcal{E})=E_{p}^{s}$ para todo $p \in \mathcal{E}$. 
Pontos Pracialmente Umbílicos Darbouxianos e semi-Darbouxianos

vii) O análogo é verdadeiro para $W^{u}(\mathcal{E})$.

Demonstração: Veja [16] e [5]. 


\section{Capítulo 3}

\section{Pontos Parcialmente Umbílicos $D_{1}^{1}$, $D_{2}^{1}, D_{3}^{1}, D_{13}^{1}, D_{1 h, p}^{1}$ e $D_{1 h, n}^{1}$}

Neste capítulo, estudaremos as linhas de curvatura em torno de pontos parcialmente umbílicos regulares (veja definição 4 no capítulo 1 ) nos quais as condições $D_{12}$ e $D_{23}$ são infringidas da maneira mais simples possível.

Lembramos que, na carta de Monge reduzida com $k_{3}=1$, as condições para que um ponto parcialmente umbílico seja do tipo $D_{12}$ ou $D_{23}$ são, respectivamente,

$\left.D_{12}\right) c b(b-a) \neq 0, \frac{a}{b}=2$ e $\chi_{2} \neq 0$. onde

$$
\begin{aligned}
\chi_{2} & =\frac{1}{27 b^{6}(k-1)}\left[q_{111} b C+q_{201} B b+2 q_{111} q_{201} b q_{021}+2 q_{111} q_{102} b^{2}+\right. \\
& +q_{111} B c-3 q_{201} q_{111}^{2} c-2 q_{111}^{3} b-Q_{211} b^{2}-3 q_{111} q_{201}^{2} b-B b q_{021}+ \\
& +q_{201} q_{012} b^{2}+\left(B b q_{021}+Q_{211} b^{2}-q_{201} B b-q_{111} B c-q_{111} b C\right) k- \\
& \left.-q_{111} b k^{3}+q_{111} b k^{4}\right] c^{3}
\end{aligned}
$$

$\left.D_{23}\right) b=a \neq 0, \chi=c B-\left(C-A+2 k^{3}\right) b+\frac{-2 q_{111}^{2} b+2 q_{201}^{2} b+2 q_{201} q_{111} c}{b(k-1)} \neq 0 \mathrm{e}$ $-b\left(-q_{201}+q_{021}\right)-c q_{111} \neq 0$.

Assim, violando as condições $D_{12}$ e $D_{23}$ da maneira mais simples possível obtemos os seguintes novos tipos: 
Pontos Parcialmente Umbílicos $D_{1}^{1}, D_{2}^{1}, D_{3}^{1}, D_{13}^{1}, D_{1 h, p}^{1}$ e $D_{1 h, n}^{1}$

$\left.D_{1}^{1}\right)$ Se $c=0, a=2 b \neq 0$ então $\tilde{\chi}_{1} \neq 0$, onde

$$
\begin{aligned}
\tilde{\chi}_{1}= & q_{111} k^{4}-q_{111} k^{3}+Q_{211} b k+q_{021} B k-q_{201} B k-q_{111} C k-q_{021} B \\
& -2 q_{111}{ }^{3}+b q_{012} q_{201}-3 q_{201}{ }^{2} q_{111}+q_{111} C-Q_{211} b+q_{201} B \\
& +2 q_{111} b q_{102}+2 q_{201} q_{111} q_{021}
\end{aligned}
$$

$\left.D_{2}^{1}\right)$ Se $b=a \neq 0, \chi=0$ então $\tilde{\chi}<0$,

$\left.D_{3}^{1}\right)$ Se $b=a \neq 0, \chi=0$ então $\tilde{\chi}>0$, onde $\chi$ é o coeficiente de $u^{2}$ na primeira equação de $(2.31)$ e $\tilde{\chi}$ o de $u^{3}$.

$\left.D_{13}^{1}\right)$ Se $a=b=0$ então $c q_{111} \neq 0$ e $\frac{B k-B+3 q_{111} q_{201}}{k-1} \neq 0$, ou se $c=b=0$ então $a q_{111} \neq 0$ e $\frac{B k-B+3 q_{111} q_{201}}{k-1} \neq 0$,

$\left.D_{1 h, p}^{1}\right)$ Se $c b(b-a) \neq 0, \frac{a}{b}=2, \chi_{2}=0$ e $\tilde{\chi}_{2}>0$

$\left.D_{1 h, n}^{1}\right)$ Se $c b(b-a) \neq 0, \frac{a}{b}=2, \chi_{2}=0$ e $\tilde{\chi}_{2}<0$.

No capítulo 5 mostraremos que estes pontos são todos os pontos parcialmente umbílicos regulares que aparecem genericamente em famílias a um parâmetro de hipersuperfícies imersas em $\mathbb{R}^{4}$. 
Pontos Parcialmente Umbílicos $D_{1}^{1}, D_{2}^{1}, D_{3}^{1}, D_{13}^{1}, D_{1 h, p}^{1}$ e $D_{1 h, n}^{1}$

\subsection{Linhas de Curvatura numa Vizinhança de um Ponto $D_{1}^{1}$}

Seja $p \in \mathbb{M}^{3}$ um ponto parcialmente umbílico tal que $k_{1}(p)=k_{2}(p)=k(p) \neq k_{3}(p)$. Em torno de $p$, escrevemos a imersão $\alpha$ como em (1.4) onde

$$
\begin{aligned}
h\left(u_{1}, u_{2}, u_{3}\right)= & \frac{k}{2}\left(u_{1}^{2}+u_{2}^{2}\right)+\frac{k_{3}}{2} u_{3}^{2}+\frac{a}{6} u_{1}^{3}+\frac{b}{2} u_{1} u_{2}^{2}+\frac{c}{6} u_{2}^{3}+\frac{1}{6} q_{003} u_{3}^{3} \\
+ & \frac{1}{2} q_{012} u_{2} u_{3}^{2}+q_{111} u_{1} u_{2} u_{3}+\frac{1}{2} q_{021} u_{2}^{2} u_{3}+\frac{1}{2} q_{102} u_{1} u_{3}^{2} \\
& +\frac{1}{2} q_{201} u_{1}^{2} u_{3}+\frac{1}{24} A u_{1}^{4}+\frac{1}{6} B u_{1}^{3} u_{2}+\frac{1}{4} C u_{1}^{2} u_{2}^{2}+\frac{1}{6} D u_{1} u_{2}^{3} \\
& +\frac{1}{24} E u_{2}^{4}+\frac{1}{24} Q_{004} u_{3}^{4}+\frac{1}{6} Q_{013} u_{3}^{3} u_{2}+\frac{1}{6} Q_{103} u_{3}^{3} u_{1} \\
& +\frac{1}{4} Q_{022} u_{2}^{2} u_{3}^{2}+\frac{1}{4} Q_{202} u_{1}^{2} u_{3}^{2}+\frac{1}{2} Q_{112} u_{3}^{2} u_{1} u_{2}+\frac{1}{6} Q_{031} u_{3} u_{2}^{3} \\
& +\frac{1}{6} Q_{301} u_{1}^{3} u_{3}+\frac{1}{2} Q_{121} u_{3} u_{2}^{2} u_{1}+\frac{1}{2} Q_{211} u_{3} u_{2} u_{1}^{2}+O(5)
\end{aligned}
$$

Via homotetia podemos supor, sem perda de generalidade, que $k_{3}(p)=1$.

Definição 8 (Ponto parcialmente umbílico do tipo $D_{1}^{1}$ ) Sejam $p \in \mathbb{M}^{3}$ um ponto parcialmente umbílico tal que $k_{1}(p)=k_{2}(p)=k(p) \neq 1$ e $\left(u_{1}, u_{2}, u_{3}\right): \mathbb{M}^{3} \rightarrow \mathbb{R}^{3}$ a carta de Monge reduzida, onde $h\left(u_{1}, u_{2}, u_{3}\right)$ é como dada em (3.2). O ponto p é chamado parcialmente umbilico do tipo $D_{1}^{1}$ se satisfaz $c=0, a=2 b \neq 0$ e $\tilde{\chi}_{1} \neq 0$, onde

$$
\begin{aligned}
\tilde{\chi}_{1}= & q_{111} k^{4}-q_{111} k^{3}+Q_{211} b k+q_{021} B k-q_{201} B k-q_{111} C k-q_{021} B \\
& -2 q_{111}{ }^{3}+b q_{012} q_{201}-3 q_{201}{ }^{2} q_{111}+q_{111} C-Q_{211} b+q_{201} B \\
& +2 q_{111} b q_{102}+2 q_{201} q_{111} q_{021}
\end{aligned}
$$

Observação 17 Iremos supor $b>0$.

Temos como objetivo, nesta seção, mostrar o seguinte teorema:

Teorema 5 Suponhamos $\alpha \in \mathcal{I}^{k}\left(\mathbb{M}^{3}, \mathbb{R}^{4}\right), k \geq 5$ e seja $p$ um ponto parcialmente umbílico do tipo $D_{1}^{1}$. Então o comportamento das linhas de curvatura, próximas à $p$, é topologicamente equivalente ao de um ponto do tipo $D_{1}$. 
Pontos Parcialmente Umbílicos $D_{1}^{1}, D_{2}^{1}, D_{3}^{1}, D_{13}^{1}, D_{1 h, p}^{1}$ e $D_{1 h, n}^{1}$

Assim como os pontos estudados por R. Garcia, esperamos que um ponto do tipo $D_{1}^{1}$ pertença a uma curva regular de pontos parcialmente umbílicos. De fato, no próximo lema veremos que a condição $b \neq 0$ não só implica que $p$ pertence a uma curva regular de pontos parcialmente umbílicos, como também implica que tal curva é transversal ao plano umbílico (plano gerado por $e_{1}(p)$ e $e_{2}(p)$, veja (1.4)).

Lema 9 Se $p$ é um ponto parcialmente umbílico do tipo $D_{1}^{1}$ então p pertence a uma curva regular c de pontos parcialmente umbílicos. Além disso, no espaço das variáveis $u_{1}, u_{2}, u_{3}$ escrevemos as coordenadas de c em função de $u_{3}$ :

$$
\left.c: \begin{array}{l}
u_{1}=c_{1}\left(u_{3}\right)=\frac{\left(-q_{201}+q_{021}\right)}{b} u_{3}+O(2) \\
u_{2}=c_{2}\left(u_{3}\right)=-\frac{q_{111}}{b} u_{3}+O(2),
\end{array} \quad \text { ( numa vizinhança da origem, }\right)
$$

ou seja, a curva parcialmente umbílica é transversal ao plano umbílico.

Demonstração: Vimos em (1.14) que os pontos parcialmente umbílicos são dados por

$$
L_{r}\left(u_{1}, u_{2}, u_{3}\right)=0 \text { e } M_{r}\left(u_{1}, u_{2}, u_{3}\right)=0 .
$$

Como, na carta de Monge reduzida,

$$
\operatorname{det}\left(\left.\frac{\partial\left(L_{r}, M_{r}\right)}{\partial\left(u_{1}, u_{2}\right)}\right|_{u_{1}=0, u_{2}=0, u_{3}=0}\right)=-b^{2} \neq 0
$$

segue-se, pelo teorema da função inversa, que $p$ pertence a uma curva regular formada por pontos parcialmente umbílicos e que, além disso, podemos escrever $u_{1}=c_{1}\left(u_{3}\right)$ e $u_{2}=c_{2}\left(u_{3}\right)$ em (3.4). Encontremos o primeiro jato de tais funções. Escrevendo

$$
\begin{aligned}
& c_{1}\left(u_{3}\right)=c_{1}^{1} u_{3} \\
& c_{2}\left(u_{3}\right)=c_{2}^{1} u_{3}
\end{aligned}
$$

e resolvendo

$$
\left\{\begin{array}{l}
\frac{\partial}{\partial u_{3}}\left[L_{r}\left(c_{1}\left(u_{3}\right), c_{2}\left(u_{3}\right), u_{3}\right)\right]=0 \\
\frac{\partial}{\partial u_{3}}\left[M_{r}\left(c_{1}\left(u_{3}\right), c_{2}\left(u_{3}\right), u_{3}\right)\right]=0
\end{array}\right.
$$

em $c_{1}^{1}$ e $c_{2}^{1}$ obtemos que

$$
\begin{gathered}
c_{1}\left(u_{3}\right)=\frac{\left(-q_{201}+q_{021}\right)}{b} u_{3}+O(2) \\
c_{2}\left(u_{3}\right) \quad=-\frac{q_{111}}{b} u_{3}+O(2)
\end{gathered}
$$


Pontos Parcialmente Umbílicos $D_{1}^{1}, D_{2}^{1}, D_{3}^{1}, D_{13}^{1}, D_{1 h, p}^{1}$ e $D_{1 h, n}^{1}$

Para determinarmos a configuração principal numa vizinhança de uma curva parcialmente umbílica $c$, usando o levantamento de Lie-Cartan definido na seção 1.2 do capítulo 1 , devemos estudar as curvas integrais de $X=\left(X_{1}, X_{2}, X_{3}, X_{4}\right)$ (restrito à hipersuperfície de Lie-Cartan) numa vizinhança dos seus equilíbrios. Na carta de Monge reduzida com $k_{3}=1, X$ é dado por

$$
\begin{aligned}
& X_{1}=\left(-2 b u_{2}-2 q_{111} u_{3}\right) P-b u_{1}+\left(-q_{201}+q_{021}\right) u_{3}+O(2) \\
& X_{2}=P X_{1} \\
& X_{3}=\left(\left(\frac{q_{111} u_{1}+q_{021} u_{2}+q_{012} u_{3}}{k-1}+O(2)\right) P+\frac{q_{201} u_{1}+q_{111} u_{2}+q_{102} u_{3}}{k-1}+O(2)\right) X_{1} \\
& X_{4}=A\left(u_{1}, u_{2}, u_{3}\right) P^{3}+B\left(u_{1}, u_{2}, u_{3}\right) P^{2}+C\left(u_{1}, u_{2}, u_{3}\right) P+D\left(u_{1}, u_{2}, u_{3}\right)
\end{aligned}
$$

com

$$
\begin{aligned}
A\left(u_{1}, u_{2}, u_{3}\right) & =b+\left(C-k^{3}+\frac{q_{111}^{2}+q_{201} q_{021}}{k-1}\right) u_{1}+\left(D+3 \frac{q_{111} q_{021}}{k-1}\right) u_{2}+ \\
& +\left(Q_{121}+\frac{2 q_{111} q_{012}+q_{102} q_{021}}{k-1}\right) u_{3}+O(2) \\
B\left(u_{1}, u_{2}, u_{3}\right)= & \left(-D+2 B+\frac{6 q_{111} q_{201}-3 q_{111} q_{021}}{k-1}\right) u_{1}+ \\
+ & \left(-E+k^{3}+2 C+\frac{4 q_{111}^{2}-3 q_{021}^{2}+2 q_{201} q_{021}}{k-1}\right) u_{2}+ \\
& +\left(-Q_{031}+2 \frac{Q_{211}\left(2 q_{201} q_{012}+4 q_{102} q_{111}-3 q_{012} q_{021}\right)}{k-1}\right) u_{3}+O(2) \\
C\left(u_{1}, u_{2}, u_{3}\right)= & \left(-2 C+A-k^{3}+\frac{-2 q_{201} q_{021}-4 q_{111}^{2}+3 q_{201}^{2}}{k-1}\right) u_{1}+ \\
+ & \left(-2 D+B+\frac{3 q_{111} q_{201}-6 q_{111} q_{021}}{k-1}\right) u_{2}+ \\
+ & \left(-2 Q_{121}+Q_{301}+\frac{3 q_{102} q_{201}-2 q_{102} q_{021}-4 q_{111} q_{012}}{k-1}\right) u_{3}+O(2) \\
+ & \left(-B-3 \frac{q_{111} q_{201}}{k-1}\right) u_{1}+\left(-C+k^{3}-\frac{2 q_{111}{ }^{2}+q_{201} q_{021}}{k-1}\right) u_{2}+ \\
D\left(u_{1}, u_{2}, u_{3}\right)= & \left.\frac{q_{201} q_{012}+2 q_{102} q_{111}}{k-1}\right) u_{3}+O(2) .
\end{aligned}
$$

No presente caso, como queremos que $c$ seja topologicamente do tipo $D_{1}$, devemos mostrar que existe uma única curva de equilíbrios $\beta$ de $X$, e que esta seja topologi- 
Pontos Parcialmente Umbílicos $D_{1}^{1}, D_{2}^{1}, D_{3}^{1}, D_{13}^{1}, D_{1 h, p}^{1}$ e $D_{1 h, n}^{1}$

camente equivalente a um conjunto normalmente hiperbólico do tipo sela (ver figura (2.6)). Tal resultado é obtido no seguinte lema:

Lema 10 Os equilíbrios do campo X, restrito à hipersuperfície de Lie-Cartan, formam uma curva regular topologicamente equivalente a um conjunto normalmente hiperbólico do tipo sela.

Demonstração: $\quad$ Sejam $\left(u_{1}, u_{2}, u_{3}\right): \mathbb{M}^{3} \rightarrow \mathbb{R}^{3}$ a carta de Monge reduzida com $k_{3}=1$ e $c\left(u_{3}\right)$ (escrita em função de $u_{3}$ pelo lema anterior) uma curva parcialmente umbílica tal que $p=c(0)$ é do tipo $D_{1}^{1}$. Suponha $u_{3}$ suficientemente pequeno. Vimos, na seção 1.2 do capítulo 1 , que os equilíbrios de $X$ são determinados pela equação:

$$
X_{4}\left(c_{1}\left(u_{3}\right), c_{2}\left(u_{3}\right), u_{3}, P\right)=0,
$$

onde $X_{4}$ é a quarta componente de $X$ e

$$
c_{1}\left(u_{3}\right)=\frac{\left(-q_{201}+q_{021}\right)}{b} u_{3}+O(2), \quad c_{2}\left(u_{3}\right)=-\frac{q_{111}}{b} u_{3}+O(2) .
$$

A equação (3.7) é cúbica em $P$ :

$$
A_{3}\left(u_{3}\right) P^{3}+A_{2}\left(u_{3}\right) P^{2}+A_{1}\left(u_{3}\right) P+A_{0}\left(u_{3}\right)=0 .
$$

onde $A_{3}(0)=b$ e $A_{2}(0)=A_{1}(0)=A_{0}(0)=0$. O discriminante desta cúbica, em função de $u_{3}$, é

$$
D\left(u_{3}\right)=\frac{1}{2} \frac{1}{b^{4}(k-1)^{2}} \tilde{\chi}_{1}^{2} u_{3}^{2}+O(3),
$$

onde $\tilde{\chi}_{1} \neq 0$ foi dada em (3.3). Disto, para $u_{3}$ suficientemente pequeno, $D\left(u_{3}\right)>0$ implicando que (3.7) possui uma única solução, que em função de $P$, pode ser escrita da forma

$$
u_{3}(P)=\frac{(k-1) b^{2}}{\tilde{\chi}} P^{3}+O(4) .
$$

Resumindo, por (3.5), (3.7) e (3.10) obtemos que, no espaço das variáveis $u_{1}, u_{2}, u_{3}$ e $P$, a curva de equilíbrios de $X$ (que denotaremos por $\beta$ ) é escrita, em função de $\mathrm{P}$, da forma:

$$
\begin{gathered}
u_{1}=\frac{\left(-q_{201}+q_{021}\right)}{b}\left(\frac{(k-1) b^{2}}{\tilde{\chi}} P^{3}\right)+O(4), \\
\beta: \quad u_{2}=-\frac{q_{111}}{b}\left(\frac{(k-1) b^{2}}{\tilde{\chi}} P^{3}\right)+O(4), \\
u_{3}=\frac{(k-1) b^{2}}{\tilde{\chi}} P^{3}+O(4) .
\end{gathered}
$$


Pontos Parcialmente Umbílicos $D_{1}^{1}, D_{2}^{1}, D_{3}^{1}, D_{13}^{1}, D_{1 h, p}^{1}$ e $D_{1 h, n}^{1}$

Agora, estudaremos o retrato de fase de $X$ restrito à hipersuperfície de Lie-Cartan, numa vizinhança da curva de equilíbrios $\beta$. Para isto, escrevemos a hipersuperfície de Lie-Cartan como gráfico de uma função $u_{2}=u_{2}\left(u_{1}, u_{3}, P\right)$. De fato, como $\frac{\partial \mathcal{L}}{\partial u_{2}}(0,0,0,0)=b \neq 0$ podemos escrever $u_{2}=u_{2}\left(u_{1}, u_{3}, P\right)$ na equação $\mathcal{L}\left(u_{1}, u_{2}, u_{3}, P\right)=$ 0 , onde

$$
\begin{aligned}
u_{2}\left(u_{1}, u_{3}, P\right)= & -\frac{q_{111}}{b} u_{3}-\frac{1}{b}\left(\frac{B}{2}+\frac{q_{201} q_{111}}{k-1}\right) u_{1}^{2}+\left(\frac{q_{201}}{b}-\frac{q_{021}}{b}\right) u_{3} P \\
& +\left(\frac{q_{201} q_{111} q_{021}}{(k-1) b^{2}}-\frac{q_{201} q_{012}}{(k-1) b}+\frac{q_{111}{ }^{3}}{(k-1) b^{2}}+\frac{q_{111} C}{b^{2}}-\frac{q_{102} q_{111}}{(k-1) b}\right. \\
& \left.-\frac{q_{111} k^{3}}{b^{2}}-\frac{Q_{211}}{b}\right) u_{1} u_{3}+\left(-\frac{q_{111}^{3} q_{021}}{(k-1) b^{3}}+\frac{2 q_{111} q_{021} q_{102}}{(k-1) b^{2}}-\frac{D q_{111}^{2}}{b^{3}}\right. \\
& \left.+\frac{q_{111}^{2} q_{012}}{(k-1) b^{2}}+\frac{2 Q_{121} q_{111}}{b^{2}}-\frac{1}{2} \frac{Q_{112}}{b}-\frac{2 q_{012} q_{102}}{b(k-1)}\right) u_{3}^{2}+u_{1} P+O(3) .
\end{aligned}
$$

Substituindo (3.12) em $X$ (veja (3.6)), obtemos a restrição do campo de Lie-Cartan à hipersuperfície de Lie-Cartan:

$$
X_{\mathcal{L}}=\left\{\begin{array}{l}
\left.\dot{u}_{1}=X_{1}\left(u_{1}, u_{2}\left(u_{1}, u_{3}, P\right), u_{3}, P\right)\right) \\
\dot{u}_{3}=X_{3}\left(u_{1}, u_{2}\left(u_{1}, u_{3}, P\right), u_{3}, P\right) \\
\dot{P}=X_{4}\left(u_{1}, u_{2}\left(u_{1}, u_{3}, P\right), u_{3}, P\right)
\end{array}\right.
$$

onde

$$
\begin{aligned}
X_{1} & =u_{3}\left(q_{021} b-q_{201} b\right)+O(2) \\
X_{3} & =\left(\frac{q_{102} b-q_{111}^{2}}{b(k-1)} u_{3}+\frac{q_{201}}{(k-1)} u_{1}+O(2)\right) X_{1} \\
X_{4} & =\left(-B-3 \frac{q_{201} q_{111}}{k-1}\right) u_{1}-b P+ \\
& +\left(-Q_{211}+\frac{q_{111} C}{b}+\frac{k^{3}}{b}-\frac{2 q_{102} q_{111}}{k-1}-\frac{q_{201} q_{012}}{k-1}+\frac{q_{201} q_{021} q_{111}}{(k-1) b}\right) u_{3}+O(2)
\end{aligned}
$$

Usando a expressão da curva de equilíbrios $\beta$, dada em (3.11), veremos que a 
Pontos Parcialmente Umbílicos $D_{1}^{1}, D_{2}^{1}, D_{3}^{1}, D_{13}^{1}, D_{1 h, p}^{1}$ e $D_{1 h, n}^{1}$

linearização de $X_{\mathcal{L}}, D X_{\mathcal{L}}(\beta(P))$, ao longo de tal curva de equilíbrios é

$$
\left(\begin{array}{ccc}
-b-2 b P^{2}+O(3) & q_{021}-q_{201}+2\left(q_{021}-q_{201}\right) P^{2}+O(3) & 0 \\
O(3) & O(3) & 0 \\
-\frac{\left(3 q_{111} b q_{201}+(k-1) b B\right)}{b(k-1)}+O(1) & -\frac{\left(q_{021}-q_{201}\right)\left(k B+3 q_{111} q_{201}-B\right)}{b(k-1)}+O(1) & 3 b P^{2}+O(3)
\end{array}\right) .
$$

Assim, teremos que $D X_{\mathcal{L}}(\beta(P))$ possui um autovalor identicamente nulo (associado à direção tangente a curva de equilíbrios) e, em função de $P$, os dois a seguir:

$$
\begin{aligned}
& \lambda_{2}(P)=3 b P^{2}+O(3) \\
& \lambda_{3}(P)=-b-2 P^{2}+O(3)
\end{aligned}
$$

donde vemos que, fora de uma vizinhança da origem, a curva de equilíbrios é normalmente hiperbólica do tipo sela. Para $P=0$ a forma de Jordan de $D X_{\mathcal{L}}(0,0,0)$ é

$$
J=\left(\begin{array}{ccc}
0 & 1 & 0 \\
0 & 0 & 0 \\
0 & 0 & -b
\end{array}\right),
$$

ou seja, $D X_{\mathcal{L}}(0,0,0)=V^{-1} J V$ onde

$$
V=\left(\begin{array}{ccc}
0 & \frac{-q_{201}+q_{021}}{b} & \frac{\tilde{\chi}_{1}}{b\left(B k-B+3 q_{111} q_{201}\right)} \\
0 & 1 & 0 \\
-\frac{\tilde{\chi}_{1}}{b(k-1)} & \frac{\tilde{\chi}_{1}}{b^{2}(k-1)} & -\frac{\tilde{\chi}_{1}}{b^{2}(k-1)}
\end{array}\right),
$$

Portanto, em $P=0$, existe uma única variedade de dimensão 2 e invariante por $X_{\mathcal{L}}, W^{s}$, onde a curva de equilíbrios é atratora, e uma variedade $W^{c}$, dita central, de dimensão 2 e invariante pelo fluxo de $X_{\mathcal{L}}$.

Nosso objetivo é estudar o fluxo de $X_{\mathcal{L}}$ restrito a $W^{c}$. Para isto, fazemos a seguinte mudança de coordenadas em (3.13)

$$
\begin{aligned}
& u_{1}=\frac{-q_{201}+q_{021}}{b} w+\frac{\tilde{\chi}_{1}}{b\left(B k-B+3 q_{111} q_{201}\right)} \bar{p} \\
& u_{3}=w \\
& P=-\frac{\tilde{\chi}_{1}}{b(k-1)} u+\frac{\tilde{\chi}_{1}}{b^{2}(k-1)} w-\frac{\tilde{\chi}_{1}}{b^{2}(k-1)} \bar{p}
\end{aligned}=V \cdot\left[\begin{array}{c}
u \\
w \\
\bar{p}
\end{array}\right],
$$

obtendo o seguinte campo:

$$
X_{J}=\left\{\begin{aligned}
\dot{u} & =w+A(u, w, \bar{p}) \\
\dot{w} & =B(u, w, \bar{p}) \\
\dot{\bar{p}} & =-b \bar{p}+C(u, w, \bar{p})
\end{aligned}\right.
$$


Pontos Parcialmente Umbílicos $D_{1}^{1}, D_{2}^{1}, D_{3}^{1}, D_{13}^{1}, D_{1 h, p}^{1}$ e $D_{1 h, n}^{1}$

onde

$$
\begin{aligned}
& \frac{\partial A}{\partial u}(0,0,0)=\frac{\partial A}{\partial w}(0,0,0)=\frac{\partial A}{\partial \bar{p}}(0,0,0)=0, \frac{\partial^{2} A}{\partial u^{2}}(0,0,0)=0, \\
& \frac{\partial^{3} A}{\partial u^{3}}(0,0,0)=\frac{\tilde{\chi}_{1}^{2}}{b(k-1)^{2}} \neq 0, \\
& \frac{\partial C}{\partial u}(0,0,0)=\frac{\partial C}{\partial w}(0,0,0)=\frac{\partial C}{\partial \bar{p}}(0,0,0)=0, \\
& B(u, w, \bar{p})=(w+A(u, w, \bar{p})) D(u, w, \bar{p}),
\end{aligned}
$$

com

$$
\begin{aligned}
D(u, w, \bar{p}) & =\left(\frac{q_{102} b-q_{111}^{2}}{b(k-1)}+\frac{q_{201}}{(k-1)} \frac{-q_{201}+q_{021}}{b}\right) w+ \\
& +\frac{\tilde{\chi}_{1}}{b\left(B k-B+3 q_{111} q_{201}\right)} \bar{p}+O(2) .
\end{aligned}
$$

Nas coordenadas $u, w$ e $\bar{p}$ a variedade central, $W^{c}$, é tangente ao plano $u w$, isto implica que podemos parametrizar $W^{c}$ por $\bar{p}=\bar{p}(u, w)$.

Obteremos a expressão de $\bar{p}(u, w)$ até segunda ordem, em funcão dos coeficientes de $X_{J}$. Para isto, escrevemos

$$
\bar{p}(u, w)=p_{20} u^{2}+p_{11} u w+p_{02} w^{2}+O(3) .
$$

Como $\bar{p}(u, w)$ satisfaz a última equação de (3.16) segue-se que

$$
\frac{\partial \bar{p}}{\partial u} \dot{u}+\frac{\partial \bar{p}}{\partial w} \dot{w}=-b \bar{p}(u, w)+C(u, w, \bar{p}(u, w)),
$$

Escrevendo

$$
C(u, w, \bar{p})=c_{200} u^{2}+c_{020} w^{2}+c_{002} \bar{p}^{2}+c_{110} u w+c_{101} u \bar{p}+c_{011} w \bar{p}+O(3)
$$

e considerando apenas os termos de ordem 2 em (3.18), teremos

$$
w\left(2 p_{20} u+p_{11} w\right)=-b\left(p_{20} u^{2}+p_{11} u w+p_{02} w^{2}\right)+c_{200} u^{2}+c_{020} w^{2}+c_{110} u w
$$

implicando que

$$
\left(c_{200}-b p_{20}\right) u^{2}+\left(2 p_{20}-p_{11}+c_{110}\right) u w+\left(p_{11}-b p_{02}+c_{020}\right) w^{2}=0 .
$$

Disto

$$
p_{20}=\frac{c_{200}}{b}, \quad p_{11}=\frac{c_{110}}{b}+\frac{2 c_{200}}{b^{2}}, \quad p_{02}=\frac{c_{020}}{b}+\frac{c_{110}}{b^{2}}+\frac{2 c_{200}}{b^{3}},
$$

donde obtemos que a variedade central associada a $X_{J}$ é dada por

$$
\bar{p}(u, w)=\frac{c_{200}}{b} u^{2}+\left(\frac{c_{110}}{b}+\frac{2 c_{200}}{b^{2}}\right) u w+\left(\frac{c_{020}}{b}+\frac{c_{110}}{b^{2}}+\frac{2 c_{200}}{b^{3}}\right) w^{2}+O(3) .
$$


Pontos Parcialmente Umbílicos $D_{1}^{1}, D_{2}^{1}, D_{3}^{1}, D_{13}^{1}, D_{1 h, p}^{1}$ e $D_{1 h, n}^{1}$

Observação 18 Observamos que $c_{200}=\frac{1}{2} \frac{\partial^{2} C}{\partial u^{2}}(0,0,0), c_{110}=\frac{\partial^{2} C}{\partial u w}(0,0,0)$ e $c_{020}=$ $\frac{1}{2} \frac{\partial^{2} C}{\partial w^{2}}(0,0,0)$, veja as equações (3.16) e (3.19).

Substituindo (3.21) em (3.16), obtemos que a restrição de $X_{J}$ à variedade central é dado por

$$
X_{c}=\left\{\begin{array}{rl}
\dot{u} & =w+\tilde{A}(u, w) \\
\dot{w} & =\tilde{B}(u, w)
\end{array} .\right.
$$

onde

$$
\tilde{A}(u, w)=A(u, w, \bar{p}(u, w)), \quad \tilde{B}(u, w)=B(u, w, \bar{p}(u, w))
$$

e

$$
\begin{aligned}
& \frac{\partial \tilde{A}}{\partial u}(0,0)=\frac{\partial \tilde{A}}{\partial w}(0,0)=0, \frac{\partial^{2} \tilde{A}}{\partial u^{2}}(0,0)=0, \frac{\partial^{3} \tilde{A}}{\partial u^{3}}(0,0)=\frac{\tilde{\chi}_{1}^{2}}{b(k-1)^{2}} \neq 0 \\
& B(u, w, \bar{p})=(w+\tilde{A}(u, w)) D(u, w, \bar{p}(u, w)) .
\end{aligned}
$$

No campo $X_{c}$, a origem é uma singularidade nilpotente, esta foi estudada em [3], onde é obtido que o retrato de fase de $X_{c}$ é como na figura 3.1.

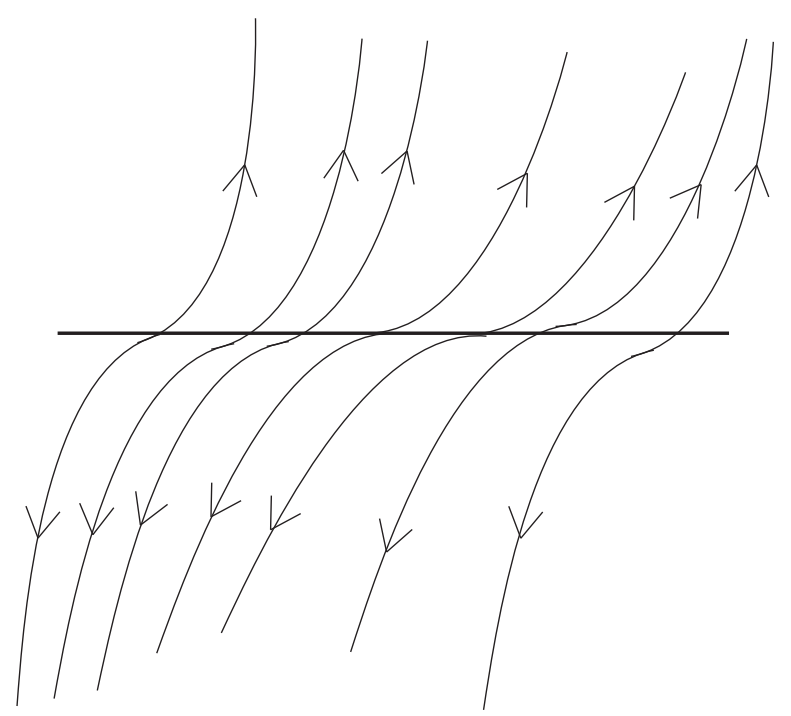

Figura 3.1: Retrato de fase de $X_{c}$. 
Pontos Parcialmente Umbílicos $D_{1}^{1}, D_{2}^{1}, D_{3}^{1}, D_{13}^{1}, D_{1 h, p}^{1}$ e $D_{1 h, n}^{1}$

Portanto $X_{J}$ tem retrato de fase, numa vizinhança da origem, como mostrado na figura 3.2 .

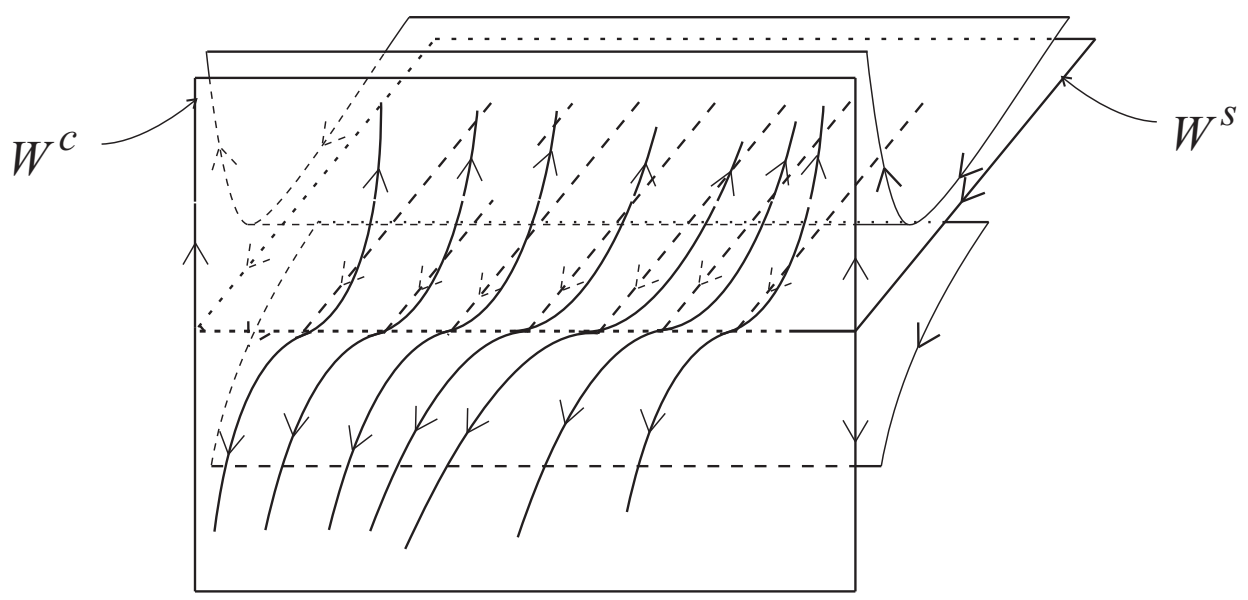

Figura 3.2: Retrato de fase de $X_{J}$.

Logo o retrato de fase na figura 3.2 é topologicamente equivalente ao retrato de fase numa vizinhança de uma curva normalmente hiperbólica do tipo sela.

Prova do teorema 5: Seja $p$ um ponto parcialmente umbílico do tipo $D_{1}^{1}$.

Pelo lema 9, $p$ pertence a uma curva regular $c$ formada por pontos parcialmente umbílicos.

Pelo lema 10, o campo de Lie-Cartan, restrito a hipersuperfície de Lie-Cartan, possui uma única curva de equilíbrios e esta é topologicamente equivalente a uma curva normalmente hiperbólica do tipo sela.

Seja $\Pi\left(u_{1}, u_{2}, u_{3}, P\right)=\left(u_{1}, u_{2}, u_{3}\right)$. Vimos na seção 1.2 do capítulo 1 que $\Pi$ projeta as curvas integrais de $X_{\mathcal{L}}$, numa vizinhança de $\beta$, nas linhas de curvatura de $\alpha$, numa vizinhança de $c$. Sendo $\beta$ topologicamente equivalente a uma curva normalmente hiperbólica do tipo sela, segue-se que $c=\Pi(\beta)$ é topologicamente equivalente a uma curva parcialmente umbílica do tipo $D_{1}$. Veja figura 2.6 no capítulo 2. 
Pontos Parcialmente Umbílicos $D_{1}^{1}, D_{2}^{1}, D_{3}^{1}, D_{13}^{1}, D_{1 h, p}^{1}$ e $D_{1 h, n}^{1}$

\subsection{Linhas de Curvatura numa Vizinhança dos Pon- tos Parcialmente Umbílicos dos Tipos $D_{2}^{1}$ e $D_{3}^{1}$}

Sejam $p \in \mathbb{M}^{3}$ um ponto parcialmente umbílico tal que $k_{1}(p)=k_{2}(p)=k(p) \neq k_{3}(p)$ e $\left(u_{1}, u_{2}, u_{3}\right): \mathbb{M}^{3} \rightarrow \mathbb{R}^{3}$ a carta de Monge reduzida, definida em (1.4), onde

$$
\begin{aligned}
h\left(u_{1}, u_{2}, u_{3}\right)= & \frac{k}{2}\left(u_{1}^{2}+u_{2}^{2}\right)+\frac{k_{3}}{2} u_{3}^{2}+\frac{a}{6} u_{1}^{3}+\frac{b}{2} u_{1} u_{2}^{2}+\frac{c}{6} u_{2}^{3}+\frac{q_{003}}{6} u_{3}^{3} \\
& +\frac{1}{2} q_{012} u_{2} u_{3}^{2}+q_{111} u_{1} u_{2} u_{3}+\frac{1}{2} q_{021} u_{2}^{2} u_{3}+\frac{1}{2} q_{102} u_{1} u_{3}^{2} \\
& +\frac{1}{2} q_{201} u_{1}^{2} u_{3}+\frac{1}{24} A u_{1}^{4}+\frac{1}{6} B u_{1}^{3} u_{2}+\frac{1}{4} C u_{1}^{2} u_{2}^{2}+\frac{1}{6} D u_{1} u_{2}^{3} \\
& +\frac{1}{24} E u_{2}^{4}+\frac{1}{24} Q_{004} u_{3}^{4}+\frac{1}{6} Q_{013} u_{3}^{3} u_{2}+\frac{1}{6} Q_{103} u_{3}^{3} u_{1} \\
& +\frac{1}{4} Q_{022} u_{2}^{2} u_{3}^{2}+\frac{1}{4} Q_{202} u_{1}^{2} u_{3}^{2}+\frac{1}{2} Q_{112} u_{3}^{2} u_{1} u_{2}+\frac{1}{6} Q_{031} u_{3} u_{2}^{3} \\
& +\frac{1}{6} Q_{301} u_{1}^{3} u_{3}+\frac{1}{2} Q_{121} u_{3} u_{2}^{2} u_{1}+\frac{1}{2} Q_{211} u_{3} u_{2} u_{1}^{2}+ \\
& +\frac{1}{120} a_{500} u_{1}^{5}+\frac{1}{24} a_{410} u_{1}^{4} u_{2}+\frac{1}{12} a_{320} u_{1}^{3} u_{2}^{2}+\frac{1}{12} a_{230} u_{1}^{2} u_{2}^{3} \\
& +\frac{1}{4} a_{122} u_{1} u_{2}^{2} u_{3}^{2}+O(6) \\
& +\frac{1}{24} a_{140} u_{1} u_{2}^{4}+\frac{1}{120} a_{050} u_{2}^{5}+\frac{1}{24} a_{401} u^{4} u_{3}+\frac{1}{12} a_{302} u_{1}^{3} u_{3}^{2} \\
& +\frac{1}{12} a_{203} u_{1}^{2} u_{3}^{3}+\frac{1}{24} a_{104} u_{1} u_{3}^{4}+\frac{1}{120} a_{005} u_{3}^{5}+\frac{1}{24} a_{041} u_{2}^{4} u_{3} \\
& +\frac{1}{12} a_{032} u_{2}^{3} u_{3}^{2}+\frac{1}{12} a_{023} u_{2}^{2} u_{3}^{3}+\frac{1}{24} a_{014} u_{2} u_{3}^{4}+\frac{1}{6} a_{311} u_{1}^{3} u_{2} u_{3} u_{2} u_{3}^{3}+\frac{1}{4} a_{221} u_{1}^{2} u_{2}^{2} u_{3}+\frac{1}{4} a_{212} u_{1}^{2} u_{2} u_{3}^{2} \\
& +\frac{1}{6}{ }^{2} \\
& \\
& \\
&
\end{aligned}
$$


Pontos Parcialmente Umbílicos $D_{1}^{1}, D_{2}^{1}, D_{3}^{1}, D_{13}^{1}, D_{1 h, p}^{1}$ e $D_{1 h, n}^{1}$

Definição 9 (Ponto parcialmente umbílico $D_{2}^{1}$ e $D_{3}^{1}$ ) O ponto p é chamado parcialmente umbílico do tipo $D_{2}^{1}$, respectivamente $D_{3}^{1}$, se satisfaz $b=a \neq 0, c q_{111}+b q_{201}-$ $b q_{021} \neq 0, \chi=0$ e $\tilde{\chi}<0$, respectivamente $\tilde{\chi}>0$, onde

$$
\chi=C-A-\frac{c B}{b}-2 \frac{c q_{111} q_{201}}{(k-1) b}+2 k^{3}-\frac{q_{201}^{2}-2 q_{111}^{2}}{k-1}
$$

$e$

$$
\begin{aligned}
\tilde{\chi} & =\frac{a_{410} c}{b}-a_{500}+a_{320}-\frac{3 D B}{b}+\frac{3 c C B}{b^{2}}+\frac{3 B^{2}}{b}+12 k^{2} b-\left(\frac{3 c B}{b^{2}}-\frac{6 q_{201} c q_{111}}{(k-1) b^{2}}\right) k^{3}+ \\
& +\frac{6 q_{201}^{2} b}{(k-1)^{2}}+\frac{6 q_{201}^{2} c q_{111} q_{021}}{(k-1)^{2} b^{2}}+\frac{6 Q_{211} q_{111}}{k-1}+\frac{3 c B q_{111}^{2}}{(k-1) b^{2}}+\frac{6 q_{201} q_{111} c}{(k-1)^{2}}+ \\
& +\frac{12 q_{111}^{2} q_{201}^{2}}{(k-1)^{2} b}-\frac{6 q_{201} q_{111} D}{(k-1) b}-\frac{6 q_{201}^{2} q_{102}}{(k-1)^{2}}+\frac{3 q_{201} c B q_{021}}{(k-1) b^{2}}+\frac{12 q_{201} B q_{111}}{(k-1) b}+ \\
& +\frac{6 q_{201} c q_{111}^{3}}{(k-1)^{2} b^{2}}-\frac{12 q_{021} q_{111}^{2} q_{201}}{(k-1)^{2} b}-\frac{6 q_{201} q_{102} c q_{111}}{(k-1)^{2} b}+\frac{6 q_{102} q_{111}^{2}}{(k-1)^{2}}-\frac{3 q_{201} c Q_{211}}{(k-1) b}+ \\
& +\frac{6 q_{201} q_{111} c C}{(k-1) b^{2}}-\frac{3 c q_{111} Q_{301}}{(k-1) b}-\frac{6 q_{111}^{2} b}{(k-1)^{2}}-\frac{6 q_{021} q_{111} B}{(k-1) b}
\end{aligned}
$$

Observação 19 Lembramos que $\chi$ e $\tilde{\chi}$ são os coeficientes de $u_{1}^{2}$ e de $u_{1}^{3}$, respectivamente, no campo de Lie-Cartan restrito a hipersuperfície de Lie-Cartan, veja (3.27) e (3.29).

Nosso objetivo, nesta seção, é mostrar o seguinte teorema:

Teorema 6 Suponhamos $\alpha \in \mathcal{I}^{k}\left(\mathbb{M}^{3}, \mathbb{R}^{4}\right), k \geq 5$ e seja $p$ um ponto parcialmente umbílico do tipo $D_{2}^{1}$, respectivamente $D_{3}^{1}$. Então o comportamento das linhas de curvatura, próximas à $p$, é topologicamente equivalente a uma curva parcialmente umbílica do tipo $D_{2}$, respectivamente $D_{3}$, se $\tilde{\chi}>0$, respectivamente $\tilde{\chi}<0$.

Como na seção anterior, começamos verificando que

$$
\operatorname{det}\left(\left.\frac{\partial\left(L_{r}, M_{r}\right)}{\partial\left(u_{2}, u_{3}\right)}\right|_{u_{1}=0, u_{2}=0, u_{3}=0}\right)=c q_{111}+b q_{201}-b q_{021} \neq 0
$$


Pontos Parcialmente Umbílicos $D_{1}^{1}, D_{2}^{1}, D_{3}^{1}, D_{13}^{1}, D_{1 h, p}^{1}$ e $D_{1 h, n}^{1}$

implica que $p$ pertence a uma curva regular $c$ formada por pontos parcialmente umbílicos. Além disso, pelo teorema da função implícita, podemos escrever $u_{2}=c_{2}\left(u_{1}\right)$ e $u_{3}=c_{3}\left(u_{1}\right) \mathrm{em}$

$$
L_{r}\left(u_{1}, u_{2}, u_{3}\right)=0, \quad M_{r}\left(u_{1}, u_{2}, u_{3}\right)=0 .
$$

Tais funções satisfazem:

$$
\begin{aligned}
& c_{2}\left(u_{1}\right)=\frac{1}{2}\left(\frac{A}{c}+2 \frac{q_{201}{ }^{2}}{(k-1) c}-\frac{C}{c}-2 k^{3} c-2 \frac{q_{111}^{2}}{(k-1) c}\right) u_{1}^{2}+O(3) \\
& c_{3}\left(u_{1}\right)=-\frac{b \chi}{-c q_{111}+b q_{021}-b q_{201}} u_{1}^{2}+O(3)
\end{aligned}
$$

Seja $X_{\mathcal{L}}$ o campo de Lie-Cartan restrito a hipersuperfície de Lie-Cartan. Para provarmos o teorema 6 , usando o levantamento de Lie-Cartan, devemos mostrar que $c$ é a projeção de três curvas de equilíbrios do tipo sela, para o caso $D_{3}$, ou uma do tipo nó entre duas do tipo sela, para o caso $D_{2}$. O próximo lema garante a existência das 3 curvas de equilíbrios e o lema 12 determina o comportamento das curvas integrais de $X_{\mathcal{L}}$ numa vizinhança de tais curvas de equilíbrios.

Lema 11 Sejam $\left(u_{1}, u_{2}, u_{3}\right): \mathbb{M}^{3} \rightarrow \mathbb{R}^{3}$ a carta de Monge, X o campo de Lie-Cartan e c $\left(u_{1}\right)$ uma curva parcialmente umbílica tal que $p=c(0)$ é do tipo $D_{2}^{1}$ ou $D_{3}^{1}$. Para $u_{1}$ suficientemente pequeno, existem exatamente três curvas, $\beta_{i}(i=1,2,3)$, de equilíbrios de $X$. No espaço das variáveis $u_{1}, u_{2}, u_{3}, P$ escrevemos $\beta_{i}: u_{2}=c_{2}\left(u_{1}\right), u_{3}=c_{3}\left(u_{1}\right)$ e $P=P_{i}\left(u_{1}\right)(i=1,2,3)$ onde

$$
\begin{aligned}
& P_{1}\left(u_{1}\right)=0+O(1) ; \\
& P_{2}\left(u_{1}\right)=\frac{1}{2} \frac{c+\sqrt{c^{2}+4 b^{2}}}{b}+O(1) ; \\
& P_{3}\left(u_{1}\right)=\frac{1}{2} \frac{c-\sqrt{c^{2}+4 b^{2}}}{b}+O(1) .
\end{aligned}
$$

Demonstração: Na seção 1.2 do capítulo 1, vimos que os equilíbrios de $X$ são dados por

$$
\left\{\begin{array}{l}
\left\{\begin{array}{l}
L_{r}\left(u_{1}, u_{2}, u_{3}\right)=0 \\
M_{r}\left(u_{1}, u_{2}, u_{3}\right)=0
\end{array}\right. \\
\left(\mathcal{L}_{u_{1}}+P \mathcal{L}_{u_{2}}+\mathcal{L}_{u_{3}}(\mathcal{U}+\mathcal{V} P)\right)\left(u_{1}, u_{2}, u_{3}, P\right)=0
\end{array}\right.
$$

Sendo as coordenadas da curva parcialmente umbílica escritas em função de $u_{1}$ : $u_{2}=c_{2}\left(u_{1}\right)$ e $u_{3}=c_{3}\left(u_{1}\right)$, segue-se que os equilíbrios de $X$ são dados por:

$$
\left(\mathcal{L}_{u_{1}}+P \mathcal{L}_{u_{2}}+\mathcal{L}_{u_{3}}(\mathcal{U}+\mathcal{V} P)\right)\left(u_{1}, c_{2}\left(u_{1}\right), c_{3}\left(u_{1}\right), P\right)=0
$$


Pontos Parcialmente Umbílicos $D_{1}^{1}, D_{2}^{1}, D_{3}^{1}, D_{13}^{1}, D_{1 h, p}^{1}$ e $D_{1 h, n}^{1}$

esta equação é cúbica em $P$ :

$$
A_{3}\left(u_{1}\right) P^{3}+A_{2}\left(u_{1}\right) P^{2}+A_{1}\left(u_{1}\right) P+A_{0}\left(u_{1}\right)=0 .
$$

onde $A_{3}(0)=b, A_{2}(0)=-c, A_{1}(0)=-b$ e $A_{0}(0)=0$. Usando a carta de Monge reduzida, o discriminante $D\left(u_{1}\right)$ de $(3.25)$ é

$$
D\left(u_{1}\right)=-\frac{c^{2}+4 b^{2}}{108 b^{2}}+O(1)<0
$$

donde vemos que, para $u_{1}$ suficientemente pequeno, $X$ possui três curvas de equilíbrios como descrito no enunciado, já que para $u_{1}=0$, (3.25) tem raízes

$$
\begin{aligned}
& P_{1}(0)=0 ; \\
& P_{2}(0)=\frac{c+\sqrt{c^{2}+4 b^{2}}}{2 b} ; \\
& P_{3}(0)=\frac{c-\sqrt{c^{2}+4 b^{2}}}{2 b} .
\end{aligned}
$$

Lema 12 Sejam $\beta_{1}, \beta_{2}$ e $\beta_{3}$ as curvas de equilíbrios encontradas no lema 11. Restrita a hipersuperfície de Lie-Cartan, as curvas $\beta_{i}\left(u_{1}\right), i=2,3$ são normalmente hiperbólicas do tipo sela para $X_{\mathcal{L}}$. Já $\beta_{1}\left(u_{1}\right)$ é topologicamente equivalente a uma curva normalmente hiperbólico do tipo sela se $\tilde{\chi}>0$, e um atrator se $\tilde{\chi}<0$.

Demonstração: Escrevendo $\mathcal{L}=L_{r} P^{2}+M_{r} P+N_{r}$ na carta de Monge reduzida, veja (1.31), (1.32) e (1.33) no apêndice do capítulo 1 , teremos que $\frac{\partial \mathcal{L}}{\partial u_{2}}(0,0,0,0)=b \neq 0$, implicando que podemos escrever $u_{2}=u_{2}\left(u_{1}, u_{3}, P\right)$ na equação $\mathcal{L}\left(u_{1}, u_{2}, u_{3}, P\right)=0$, 
Pontos Parcialmente Umbílicos $D_{1}^{1}, D_{2}^{1}, D_{3}^{1}, D_{13}^{1}, D_{1 h, p}^{1}$ e $D_{1 h, n}^{1}$

que define a hipersuperfície de Lie-Cartan. Tal função é dada por:

$$
\begin{aligned}
u_{2}\left(u_{1}, u_{3}, p\right)= & -\frac{q_{111}}{b} u_{3}-\frac{B}{2 b} u_{1}^{2}+\left(-\frac{Q_{211}}{b}-\frac{3 q_{111}^{3}}{b^{2}(k-1)}+\frac{3 q_{102} q_{111}}{(k-1) b}+\frac{q_{111} C}{b^{2}}-\right. \\
& \left.-\frac{k^{3} q_{111}}{b^{2}}+\frac{\left(q_{021} q_{111}-q_{012}\right) q_{201} b}{b^{2}(k-1)}\right) u_{1} u_{3}+\left(\frac{3 q_{012} q_{111}^{2}}{(k-1) b^{2}}-\frac{3 q_{111}^{3} q_{021}}{(k-1) b^{3}}+\right. \\
& \left.+\frac{q_{111}^{2} D}{2 b^{3}}-\frac{q_{111} Q_{121}}{b^{2}}+3 \frac{q_{102} q_{021} q_{111}}{(k-1) b^{2}}+\frac{1}{2} \frac{Q_{112}}{b}-3 \frac{q_{012} q_{102}}{b(k-1)}\right) u_{3}^{2}+ \\
& +\left(\frac{q_{111} c}{b^{2}}-\frac{q_{021}}{b}+\frac{q_{201}}{b}\right) u_{3} P+\left(\frac{B C}{b^{2}}+\frac{3 q_{021} B q_{201}}{(k-1) b^{2}}+\frac{3 B q_{111}^{2}}{(k-1) b^{2}}-\right. \\
& -\frac{3 k^{3} B}{b^{2}}-\frac{3 q_{111} Q_{301}}{(k-1) b}+\frac{6 q_{111} q_{201} C}{(k-1) b^{2}}+\frac{6 q_{111} q_{201}}{(k-1)^{2}}-\frac{3 q_{201} Q_{211}}{(k-1) b}- \\
& -\frac{6 q_{111} q_{102} q_{201}}{(k-1)^{2} b}+\frac{6 q_{021} q_{111} q_{201}^{2}}{(k-1)^{2} b^{2}}+\frac{6 q_{201} q_{111}^{3}}{(k-1)^{2} b^{2}}-\frac{6 k^{3} q_{111} q_{201}}{(k-1)^{2} b^{2}}- \\
& \left.-\frac{a_{410}}{b}\right) u_{1}^{3}+\ldots
\end{aligned}
$$

Portanto, nas variáveis $u_{1}, u_{3}, P$, o campo vetorial de Lie-Cartan $X_{\mathcal{L}}$ é dado por

$$
X_{\mathcal{L}}=\left\{\begin{array}{l}
\dot{u}_{1}=X_{1}\left(u_{1}, u_{2}\left(u_{1}, u_{3}, P\right), u_{3}, P\right) \\
\dot{u}_{3}=X_{3}\left(u_{1}, u_{2}\left(u_{1}, u_{3}, P\right), u_{3}, P\right) \\
\dot{p}=X_{4}\left(u_{1}, u_{2}\left(u_{1}, u_{3}, P\right), u_{3}, P\right)
\end{array}\right.
$$

onde

$$
\begin{aligned}
& X_{1}=\frac{\left(q_{021} b-q_{111} c-b q_{201}\right)}{b} u_{3}+\chi u_{1}^{2}+\tilde{\chi} u_{1}^{3}+\ldots \\
& X_{3}\left(\frac{q_{102} b-q_{111}^{2}}{b(k-1)} u_{3}+\frac{q_{201}}{(k-1)} u_{1}+O(2)\right) X_{1} \\
& X_{4}=-B u_{1}+\left(\frac{2 q_{111}{ }^{3}}{(k-1) b}-\frac{2 q_{102} q_{111}}{k-1}+\frac{q_{111} C}{b}-\frac{k^{3} q_{111}}{b}-Q_{211}\right) u_{3}-b P+O(2)
\end{aligned}
$$

A linearização de $X_{\mathcal{L}}$, ao longo das curvas de equilíbrios $\beta_{i}\left(u_{1}\right)(i=2,3)$, possui um autovalor identicamente nulo (associado à direção tangente a curva $\beta_{i}$ ) e os seguintes dois não nulos:

$$
\begin{aligned}
& \lambda_{1}^{i}\left(u_{1}\right)=-b\left(P_{i}(0)^{2}+1\right)+O(1) \\
& \lambda_{2}^{i}\left(u_{1}\right)=b\left(P_{i}(0)^{2}+1\right)+O(1)
\end{aligned}
$$

Disto, restrito a hipersuperfície de Lie-Cartan, as curvas $\beta_{i}$ 's $(i=2,3)$ são normalmente hiperbólicas do tipo sela. 
Pontos Parcialmente Umbílicos $D_{1}^{1}, D_{2}^{1}, D_{3}^{1}, D_{13}^{1}, D_{1 h, p}^{1}$ e $D_{1 h, n}^{1}$

Agora, estudaremos o retrato de fase de $X_{\mathcal{L}}$ numa vizinhança de $\beta_{1}\left(u_{1}\right)$ para $u_{1}$ suficientemente pequeno. Sendo a forma de Jordan de $D X_{\mathcal{L}}\left(\beta_{1}(0)\right)$ a matriz

$$
J=\left(\begin{array}{ccc}
0 & 1 & 0 \\
0 & 0 & 0 \\
0 & 0 & -b
\end{array}\right)
$$

segue-se que, numa vizinhança da origem, existe uma variedade $W^{s}$ de dimensão 2 onde $\beta_{1}$ é atratora (estamos supondo $b>0$ ).

Estudaremos o retrato de fase de $X_{\mathcal{L}}$ restrito a variedade central, que neste caso tem dimensão 2 e contém a curva de equilíbrios. Como na seção anterior, depois de feita a mudança (3.15), obtemos que a restrição do campo de Lie-Cartan à variedade central é da forma (3.22), onde

$$
\frac{\partial^{2} \tilde{A}}{\partial u^{2}}(0,0)=\chi=0 \text { e } \frac{\partial^{3} \tilde{A}}{\partial u^{3}}(0,0)=\tilde{\chi} \neq 0
$$

donde vemos que, numa vizinhança de $\beta_{1}(0)$, o comportamento de $X_{\mathcal{L}}$ restrito a variedade central, $W^{c}$, é como mostrado na figura abaixo:
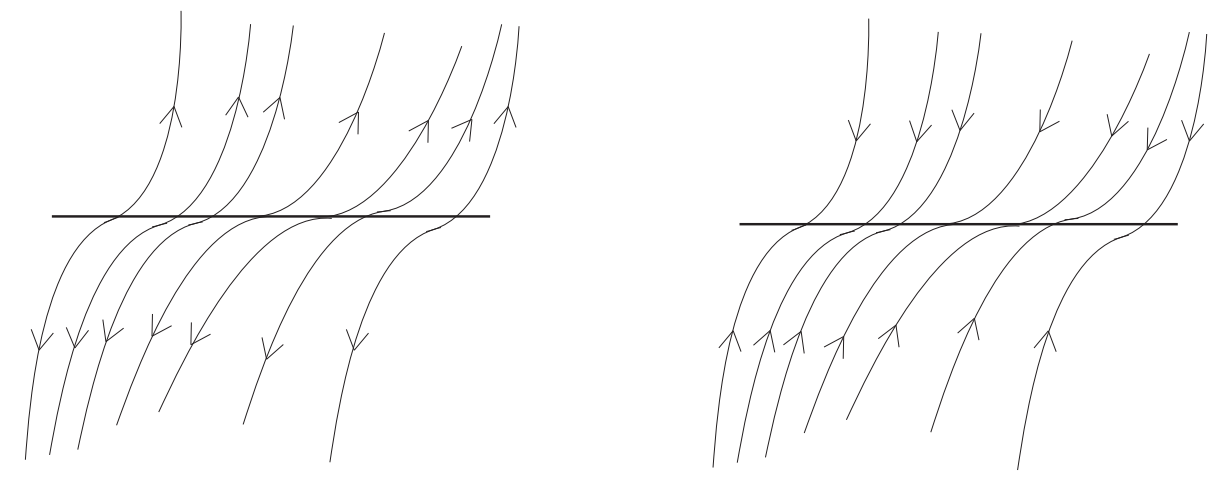

Figura 3.3: Retrato de fase de $X_{\mathcal{L}}$ restrito a variedade central para $\tilde{\chi}>0$ (esquerda) e $\tilde{\chi}<0$ (direita).

Assim, concluímos que a curva de equilíbrios é topologicamente equivalente a uma curva normalmente hiperbólica do tipo sela se $\tilde{\chi}>0$, e a um atrator se $\tilde{\chi}<0$. Como ilustração, veja figura 3.4.

Demonstração do teorema 6: Seja $p$ um ponto parcialmente umbílico do tipo $D_{2}^{1}$ ou $D_{3}^{1}$. 

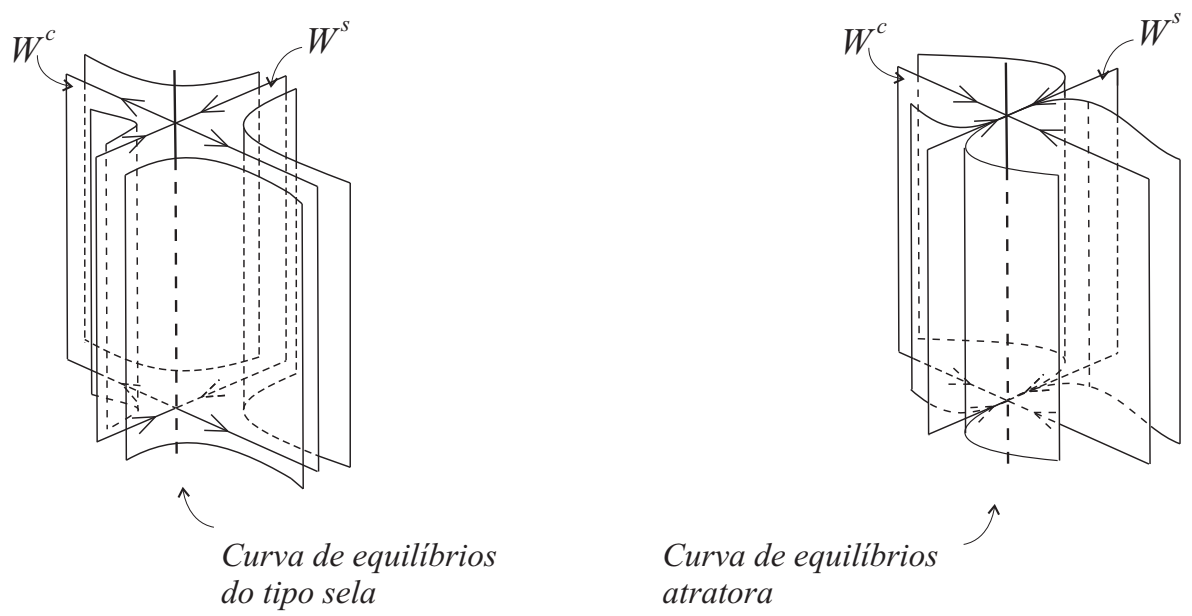

Curva de equilíbrios do tipo sela atratora

Figura 3.4: Retrato de fase de $X_{\mathcal{L}}$ numa vizinhança da curva de equilíbrios para $\tilde{\chi}>0$ (esquerda) e $\tilde{\chi}<0$ (direita).

Pela equação 3.24, $p$ pertence a uma curva regular $c$ formada por pontos parcialmente umbílicos.

Pelo lema 11, o campo de Lie-Cartan, restrito a hipersuperfície de Lie-Cartan, possui exatamente três curvas de equilíbrios $\beta_{1}, \beta_{2}$ e $\beta_{3}$.

Pelo lema 12, as curvas $\beta_{2}$ e $\beta_{3}$ são normalmente hiperbólicas do tipo sela, e $\beta_{1}$ é topologicamente equivalente a um conjunto normalmente hiperbólico de tipo sela, se $\tilde{\chi}>0$, e a um atrator se $\tilde{\chi}<0$.

Seja $\Pi\left(u_{1}, u_{2}, u_{3}, P\right)=\left(u_{1}, u_{2}, u_{3}\right)$. Vimos na seção 1.2 do capítulo 1 que $\Pi$ projeta as curvas integrais de $X$, numa vizinhança de $\beta_{1}, \beta_{2}$ e $\beta_{3}$, nas linhas de curvatura de $\alpha$, numa vizinhança de $c$. Assim,

- $\tilde{\chi}>0 \Rightarrow c$ é topologicamente equivalente a uma curva parcialmente umbílica do tipo $D_{3}$, já que no campo de Lie-Cartan, restrito a hipersuperfície de Lie-Cartan, $\beta_{1}, \beta_{2}$ e $\beta_{3}$ são do tipo sela e $\Pi\left(\beta_{i}\right)=c, i=1,2,3$.

- $\tilde{\chi}<0 \Rightarrow c$ é topologicamente equivalente a uma curva parcialmente umbílica do tipo $D_{2}$, já que o campo de Lie-Cartan, restrito a hipersuperfície de Lie-Cartan, possui um atrator, $\beta_{1}$, entre duas selas, $\beta_{2}$ e $\beta_{3}$.

Assim, concluimos a prova do teorema 6, veja figura 3.5 abaixo. 
Pontos Parcialmente Umbílicos $D_{1}^{1}, D_{2}^{1}, D_{3}^{1}, D_{13}^{1}, D_{1 h, p}^{1}$ e $D_{1 h, n}^{1}$

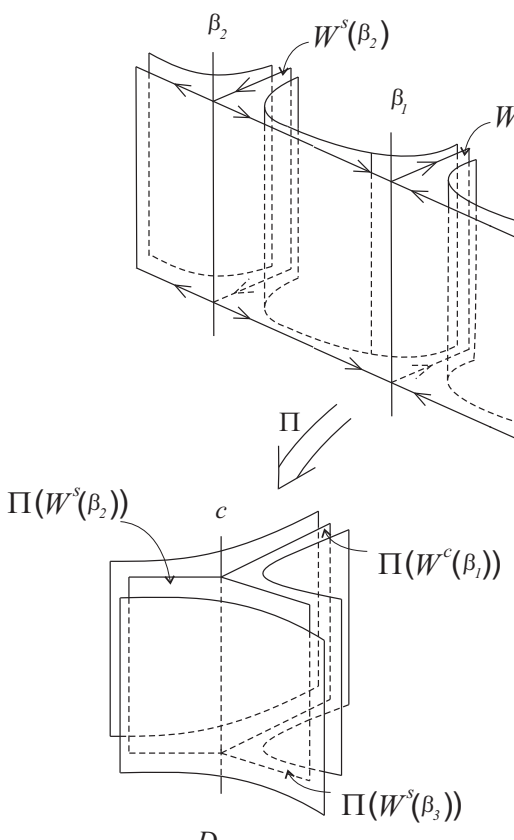

$D_{3}$

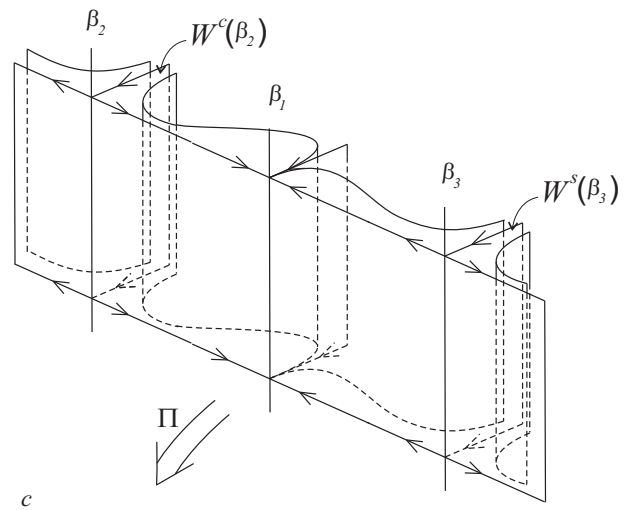

$\Pi\left(W^{s}\left(\beta_{2}\right)\right)$

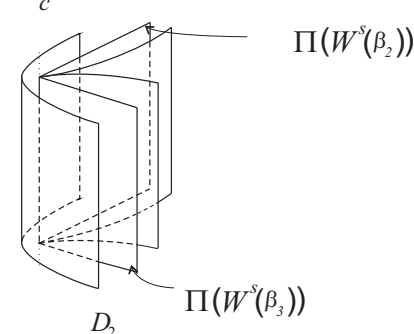

Figura 3.5: Projeção das curvas integrais do campo de Lie-Cartan na imersão $\alpha$, quando esta possui um ponto parcialmente umbílico do tipo $D_{3}^{1}$ (esquerda) ou $D_{2}^{1}$ (direita).

\subsection{Linhas de Curvatura numa Vizinhança de um Ponto $D_{13}^{1}$}

A determinação da configuração principal numa vizinhança de um ponto parcialmente umbílico do tipo $D_{13}^{1}$ é a mais trabalhosa das que vimos até agora, pois, como veremos, na singularidade do campo de Lie-Cartan, $X_{\mathcal{L}}$, correspondente a este tipo de ponto, a variedade central tem dimensão 3, ainda mais, a forma de Jordan da linearização de $X_{\mathcal{L}}$ em tal singularidade é

$$
\left(\begin{array}{lll}
0 & 1 & 0 \\
0 & 0 & 1 \\
0 & 0 & 0
\end{array}\right) .
$$

Ao longo desta seção, iremos supor que $X$ e $Y$ denotam os campos definidos em (1.17) e (1.18), respectivamente, que $p \in \mathbb{M}^{3}$ é um ponto parcialmente umbílico tal que $k_{1}(p)=k_{2}(p)=k(p) \neq k_{3}(p)$ e que $\left(u_{1}, u_{2}, u_{3}\right): \mathbb{M}^{3} \rightarrow \mathbb{R}^{3}$ é a carta de Monge reduzida, onde 
Pontos Parcialmente Umbílicos $D_{1}^{1}, D_{2}^{1}, D_{3}^{1}, D_{13}^{1}, D_{1 h, p}^{1}$ e $D_{1 h, n}^{1}$

$$
\begin{aligned}
h\left(u_{1}, u_{2}, u_{3}\right) & =\frac{k}{2}\left(u_{1}^{2}+u_{2}^{2}\right)+\frac{k_{3}}{2} u_{3}^{2}+\frac{1}{6} a u_{1}^{3}+\frac{1}{2} b u_{1} u_{2}^{2}+\frac{1}{6} c u_{2}^{3}+\frac{1}{6} q_{003} u_{3}^{3} \\
& +\frac{1}{2} q_{012} u_{2} u_{3}^{2}+q_{111} u_{1} u_{2} u_{3}+\frac{1}{2} q_{021} u_{2}^{2} u_{3}+\frac{1}{2} q_{102} u_{1} u_{3}^{2} \\
& +\frac{1}{2} q_{201} u_{1}^{2} u_{3}+\frac{1}{24} A u_{1}^{4}+\frac{1}{6} B u_{1}^{3} u_{2}+\frac{1}{4} C u_{1}^{2} u_{2}^{2}+\frac{1}{6} D u_{1} u_{2}^{3} \\
& +\frac{1}{24} E u_{2}^{4}+\frac{1}{24} Q_{004} u_{3}^{4}+\frac{1}{6} Q_{013} u_{2} u_{3}^{3}+\frac{1}{6} Q_{103} u_{1} u_{3}^{3}+\frac{1}{4} Q_{022} u_{2}^{2} u_{3}^{2} \\
& +\frac{1}{4} Q_{202} u_{1}^{2} u_{3}^{2}+\frac{1}{2} Q_{112} u_{1} u_{2} u_{3}^{2}+\frac{1}{6} Q_{031} u_{2}^{3} u_{3}+\frac{1}{6} Q_{301} u_{1}^{3} u_{3} \\
& +\frac{1}{2} Q_{121} u_{1} u_{2}^{2} u_{3}+\frac{1}{2} Q_{211} u_{1}^{2} u_{2} u_{3}+O(5) .
\end{aligned}
$$

Via homotetia iremos supor que $k_{3}(p)=1$.

Definição 10 [Ponto parcialmente umbílico $D_{13}^{1}$ / O ponto $p$ é chamado parcialmente umbílico do tipo $D_{13}^{1}$ se satisfaz

$$
a=b=0, \quad c q_{111} \neq 0 \quad e \quad \frac{B k-B+3 q_{111} q_{201}}{k-1} \neq 0
$$


Pontos Parcialmente Umbílicos $D_{1}^{1}, D_{2}^{1}, D_{3}^{1}, D_{13}^{1}, D_{1 h, p}^{1}$ e $D_{1 h, n}^{1}$

Teorema 7 Suponhamos $\alpha \in \mathcal{I}^{k}\left(\mathbb{M}^{3}, \mathbb{R}^{4}\right), k \geq 5$ e seja $p$ um ponto parcialmente umbilico do tipo $D_{13}^{1}$. Então o comportamento das linhas de curvatura próximas à $p$ é como na figura 3.6.
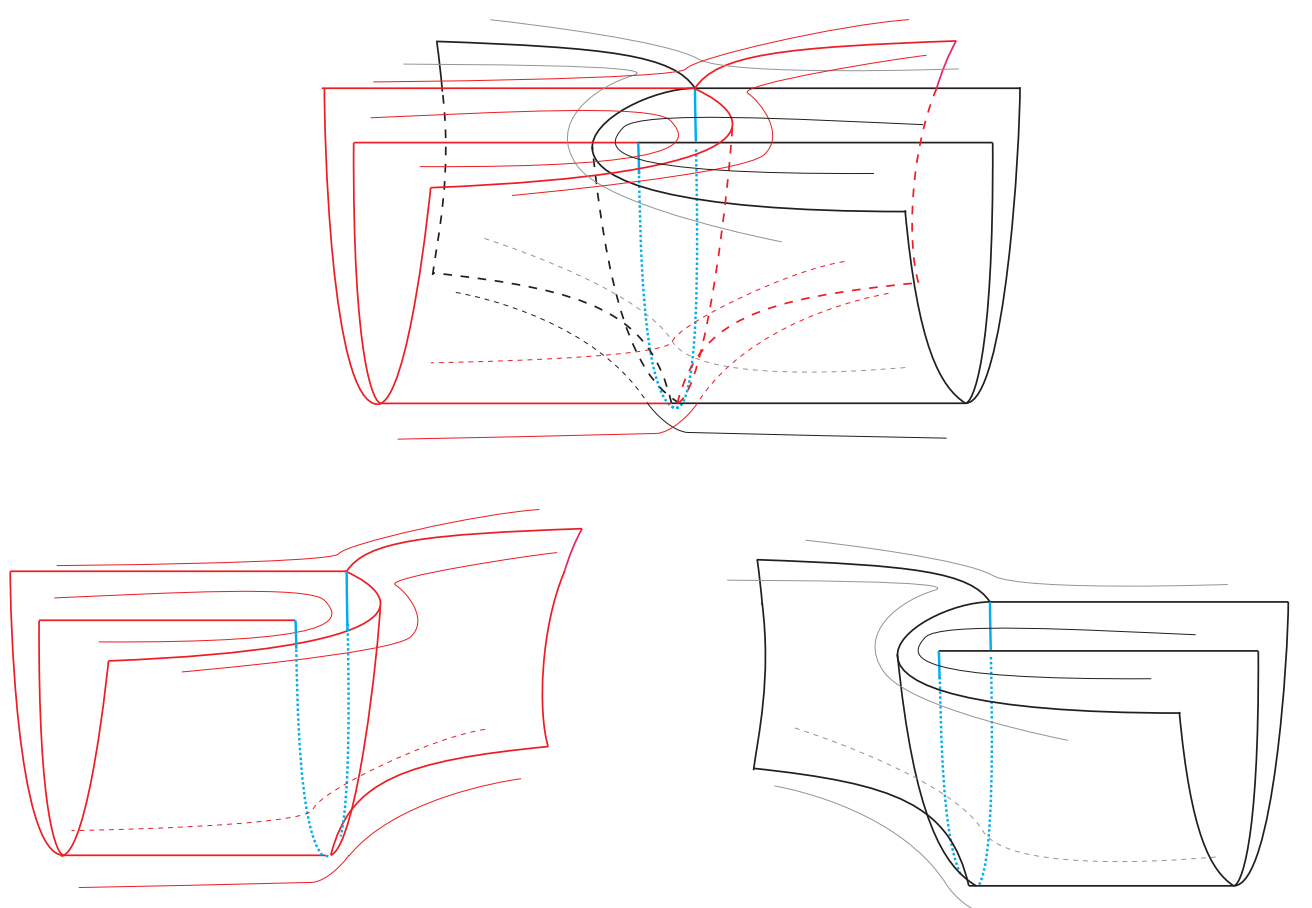

Figura 3.6: Linhas de curvatura numa vizinhança de um ponto do tipo $D_{13}^{1}$.

Observação 20 Observe que um ponto parcialmente umbilico do tipo $D_{13}^{1}$ conecta uma curva do tipo $D_{1}$ com uma curva to tipo $D_{3}$ formando uma curva regular 
Pontos Parcialmente Umbílicos $D_{1}^{1}, D_{2}^{1}, D_{3}^{1}, D_{13}^{1}, D_{1 h, p}^{1}$ e $D_{1 h, n}^{1}$

Para ver que ponto do tipo $D_{13}^{1}$ pertence a uma curva regular de pontos parcialmente umbílicos, basta observar que, na carta de Monge reduzida,

$$
\operatorname{det}\left(\left.\frac{\partial\left(L_{r}, M_{r}\right)}{\partial\left(u_{2}, u_{3}\right)}\right|_{u_{1}=0, u_{2}=0, u_{3}=0}\right)=\operatorname{det}\left(\begin{array}{cc}
0 & -q_{111} \\
c & -q_{201}+q_{021}
\end{array}\right)=c q_{111} \neq 0 .
$$

Além disso, pelo teorema da função implícita, podemos escrever $u_{2}=c_{2}\left(u_{1}\right)$ e $u_{3}=$ $c_{3}\left(u_{1}\right)$ em

$$
L_{r}\left(u_{1}, u_{2}, u_{3}\right)=0 \quad \text { e } \quad M_{r}\left(u_{1}, u_{2}, u_{3}\right)=0 .
$$

Dados

$$
\begin{aligned}
& c_{2}\left(u_{1}\right)=c_{2}^{1} u_{1}+c_{2}^{2} u_{1}^{2} \\
& c_{3}\left(u_{1}\right)=c_{3}^{1} u_{1}+c_{3}^{2} u_{1}^{2}
\end{aligned}
$$

e resolvendo

$$
\left\{\begin{array}{c}
\left.\frac{\partial}{\partial u_{1}}\left[L_{r}\left(u_{1}, c_{2}\left(u_{1}\right), c_{3}\left(u_{1}\right)\right)\right]\right|_{u_{1}=0}=0 \\
\left.\left.\frac{\partial}{\partial u_{1}}\left[M_{r}\left(u_{1}, c_{2}\left(u_{1}\right), c_{3}\left(u_{1}\right)\right)\right]\right|_{u_{1}=0}\right|_{u_{1}=0}=0 \\
\left.\frac{\partial^{2}}{\partial u_{1}^{2}}\left[L_{r}\left(u_{1}, c_{2}\left(u_{1}\right), c_{3}\left(u_{1}\right)\right)\right]\right|_{u_{1}=0}=0 \\
\left.\frac{\partial^{2}}{\partial u_{1}^{2}}\left[M_{r}\left(u_{1}, c_{2}\left(u_{1}\right), c_{3}\left(u_{1}\right)\right)\right]\right|_{u_{1}=0}=0
\end{array}\right.
$$

em $c_{2}^{1}, c_{2}^{2}, c_{3}^{1}$ e $c_{3}^{2}$, obtemos que

$$
\begin{aligned}
& c_{2}^{1}=0=c_{3}^{1} \\
& c_{2}^{2}=\frac{A}{2 c}-\frac{C}{2 c}+\frac{\left(-q_{201}+q_{021}\right) B}{2 q_{111} c}-\frac{q_{111}^{2}}{(k-1) c}+\frac{-k^{3}}{c}+\frac{q_{201} q_{021}}{(k-1) c} \\
& c_{3}^{2}=-\frac{B}{2 q_{111}}-\frac{q_{201}}{k-1},
\end{aligned}
$$

donde vemos que as cordenadas $u_{2}$ e $u_{3}$, da curva parcialmente umbílica que contém um ponto do tipo $D_{13}^{1}$ podem ser escrita, numa vizinhança do ponto $p$, em função de $u_{1}: u_{2}=c_{2}\left(u_{1}\right)$ e $u_{3}=c_{3}\left(u_{1}\right)$ onde

$$
\begin{aligned}
c_{2}\left(u_{1}\right)= & \left(\frac{A}{2 c}-\frac{C}{2 c}+\frac{\left(-q_{201}+q_{021}\right) B}{2 q_{111} c}-\frac{q_{111}^{2}}{(k-1) c}+\frac{-k^{3}}{c}+\right. \\
& \left.+\frac{q_{201} q_{021}}{(k-1) c}\right) u_{1}^{2}+O(3) \\
c_{3}\left(u_{1}\right)= & \left(-\frac{B}{2 q_{111}}-\frac{q_{201}}{k-1}\right) u_{1}^{2}+O(3)
\end{aligned}
$$


Pontos Parcialmente Umbílicos $D_{1}^{1}, D_{2}^{1}, D_{3}^{1}, D_{13}^{1}, D_{1 h, p}^{1}$ e $D_{1 h, n}^{1}$

Agora, usando o levantamento de Lie-Cartan, passaremos ao estudo das linhas de curvatura numa vizinhança de um curva parcialmente umbílica que contém um ponto do tipo $D_{13}^{1}$.

Lembramos que o campo de Lie-Cartan é dado por:

- Na carta $\left(u_{1}, u_{2}, u_{3}, P\right), P=\frac{d u_{2}}{d u_{1}}$ :

$$
X:=\left\{\begin{array}{l}
\dot{u}_{1}=\mathcal{L}_{P} \\
\dot{u}_{2}=P \mathcal{L}_{P} \\
\dot{u}_{3}=(\mathcal{U}+\mathcal{V} P) \mathcal{L}_{P} \\
\dot{P}=-\left(\mathcal{L}_{u_{1}}+P \mathcal{L}_{u_{2}}+\mathcal{L}_{u_{3}}(\mathcal{U}+\mathcal{V} P)\right)
\end{array}\right.
$$

onde $\mathcal{L}\left(u_{1}, u_{2}, u_{3}, P\right)=L_{r}\left(u_{1}, u_{2}, u_{3}\right) P^{2}+M_{r}\left(u_{1}, u_{2}, u_{3}\right) P+N_{r}\left(u_{1}, u_{2}, u_{3}\right)$.

- Na carta $\left(u_{1}, u_{2}, u_{3} ; Q\right), Q=\frac{d u_{1}}{d u_{2}}$ :

$$
Y:=\left\{\begin{array}{rl}
\dot{u}_{1} & =Q \frac{\partial \mathcal{G}}{\partial Q} \\
\dot{u}_{2} & =\frac{\partial \mathcal{G}}{\partial Q} \\
\dot{u}_{3} & =(\mathcal{U} Q+\mathcal{V}) \frac{\partial \mathcal{G}}{\partial Q} \\
\dot{Q} & =-\left(Q \mathcal{G}_{u_{1}}+\mathcal{G}_{u_{2}}+\mathcal{G}_{u_{3}}(\mathcal{U} Q+\mathcal{V})\right)
\end{array},\right.
$$

onde $\mathcal{G}\left(u_{1}, u_{2}, u_{3}, Q\right)=L_{r}\left(u_{1}, u_{2}, u_{3}\right)+M_{r}\left(u_{1}, u_{2}, u_{3}\right) Q+N_{r}\left(u_{1}, u_{2}, u_{3}\right) Q^{2}$.

Lema 13 Suponha $u_{1}$ suficientemente pequeno. Restrito à hipersuperficie de LieCartan, o campo $Y$ possui uma única curva de equilíbrios, $\gamma$, passando por $Q=0$. Quanto ao campo $X$, existe uma única curva de equilíbrios, $\beta$, numa vizinhança da origem, satisfazendo

$$
\begin{aligned}
u_{1}(P) & =\frac{(k-1) c}{B(k-1)+3 q_{111} q_{201}} P^{2}+O(3) \\
u_{2}(P) & =\left(\frac{A}{2 c}-\frac{C}{2 c}+\frac{\left(-q_{201}+q_{021}\right) B}{2 q_{111} c}-\frac{q_{111}{ }^{2}}{(k-1) c}+\frac{-k^{3}}{c}+\frac{q_{201} q_{021}}{(k-1) c}\right) . \\
& \cdot\left(\frac{B k-B+3 q_{111} q_{201}}{k-1}\right)^{2} P^{4}+O(5) \\
u_{3}(P) & =\left(-\frac{B}{2 q_{111}}-\frac{q_{201}}{k-1}\right)\left(\frac{B k-B+3 q_{111} q_{201}}{k-1}\right)^{2} P^{4}+O(5), .
\end{aligned}
$$


Pontos Parcialmente Umbílicos $D_{1}^{1}, D_{2}^{1}, D_{3}^{1}, D_{13}^{1}, D_{1 h, p}^{1}$ e $D_{1 h, n}^{1}$

Demonstração: Por (3.33) e (3.32) vemos que os equilíbrios de $Y$ e $X$ são, respectivamente, as soluções dos sistemas

$$
\left\{\begin{array}{l}
\mathcal{G}_{Q}\left(u_{1}, u_{2}, u_{3}\right)=0 \\
\left(Q \mathcal{G}_{u_{1}}+\mathcal{G}_{u_{2}}+\mathcal{G}_{u_{3}}(\mathcal{U} Q+\mathcal{V})\right)\left(u_{1}, u_{2}, u_{3}, Q\right)=0
\end{array}\right.
$$

e

$$
\left\{\begin{array}{l}
\mathcal{L}_{P}\left(u_{1}, u_{2}, u_{3}\right)=0 \\
\left(\mathcal{L}_{u_{1}}+P \mathcal{L}_{u_{2}}+\mathcal{L}_{u_{3}}(\mathcal{U}+\mathcal{V} P)\right)\left(u_{1}, u_{2}, u_{3}, P\right)=0
\end{array}\right.
$$

A solução da primeira equação de cada um dos sistemas são os pontos parcialmente umbílicos, que podem ser escritos em função de $u_{1}: u_{2}=c_{2}\left(u_{1}\right), u_{3}=c_{3}\left(u_{1}\right)$ (veja a equação (3.31)). Sejam

$$
\begin{aligned}
L\left(u_{1}, P\right) & =\left(\mathcal{L}_{u_{1}}+P \mathcal{L}_{u_{2}}+\mathcal{L}_{u_{3}}(\mathcal{U}+\mathcal{V} P)\right)\left(u_{1}, c_{2}\left(u_{1}\right), c_{3}\left(u_{1}\right), P\right) \\
& =A_{3}\left(u_{1}\right) P^{3}+A_{2}\left(u_{1}\right) P^{2}+A_{1}\left(u_{1}\right) P+A_{0}\left(u_{1}\right)
\end{aligned}
$$

e

$$
\begin{aligned}
G\left(u_{1}, Q\right) & =\left(Q \mathcal{G}_{u_{1}}+\mathcal{G}_{u_{2}}+\mathcal{G}_{u_{3}}(\mathcal{U} Q+\mathcal{V})\right)\left(u_{1}, c_{2}\left(u_{1}\right), c_{3}\left(u_{1}\right), Q\right) \\
& =B_{3}\left(u_{1}\right) Q^{3}+B_{2}\left(u_{1}\right) Q^{2}+B_{1}\left(u_{1}\right) Q+B_{0}\left(u_{1}\right)
\end{aligned}
$$

onde

$$
\begin{aligned}
& A_{3}\left(u_{1}, P\right)=\left(C-k^{3}+\frac{\left(2 q_{111}^{2}+q_{021} q_{201}\right)}{k-1}\right) u_{1}+O(2) \\
& A_{2}\left(u_{1}, P\right)=-c+\left(2 B-D+\frac{-3 q_{111} q_{021}+6 q_{111} q_{201}}{k-1}\right) u_{1}+O(2) \\
& A_{1}\left(u_{1}, P\right)=\left(A-2 C-k^{3}+\frac{-2 q_{021} q_{201}-4 q_{111}^{2}+3 q_{201}^{2}}{k-1}\right) u_{1}+O(2) \\
& A_{0}\left(u_{1}, P\right)=\left(-\frac{B k-B+3 q_{111} q_{201}}{k-1}\right) u_{1}+O(2) \\
& B_{3}\left(u_{1}, P\right)=\left(\frac{B-B k-3 q_{111} q_{201}}{k-1}\right) u_{1}+O(2) \\
& B_{2}\left(u_{1}, P\right)=\left(-2 C+A-\frac{k^{3}\left(-2 q_{021} q_{201}+3 q_{201}^{2}-4 q_{111}^{2}\right)}{k-1}\right) u_{1}+O(2) \\
& B_{1}\left(u_{1}, P\right)=-c+\left(-D+2 B+\frac{6 q_{111} q_{201}-3 q_{111} q_{021}}{k-1}\right) u_{1}+O(2) \\
& B_{0}\left(u_{1}, P\right)=\left(C-k^{3}+\frac{q_{021} q_{201}-k^{4}+2 q_{111}^{2}+k^{3}}{k-1}\right) u_{1}+0(2)
\end{aligned}
$$


Pontos Parcialmente Umbílicos $D_{1}^{1}, D_{2}^{1}, D_{3}^{1}, D_{13}^{1}, D_{1 h, p}^{1}$ e $D_{1 h, n}^{1}$

Como $L(0,0)=0, G(0,0)=0$,

$$
\left.\frac{\partial G}{\partial Q}\right|_{u_{1}=0=Q}=-c \neq 0
$$

$\mathrm{e}$

$$
\left.\frac{\partial L}{\partial u_{1}}\right|_{u_{1}=0=P}=-\frac{B k-B+3 q_{111} q_{201}}{k-1} \neq 0
$$

segue-se que exite uma única curva satisfazendo (3.34), respectivamente (3.35), e passando pela origem do espaço das variáveis $u_{1}, u_{2}, u_{3}$ e $Q$, respectivamente $u_{1}, u_{2}, u_{3}$ e $P$, o que conclui a primeira parte do lema.

Para determinar a parte quadrática da curva de equilíbrios do campo $X$ procedemos como segue:

Escrevendo $u_{1}(P)=u_{1}^{1} P+u_{1}^{2} P^{2}$ e resolvendo

$$
\left\{\begin{array}{l}
\left.\frac{\partial L}{\partial P}\left(u_{1}(P), P\right)\right|_{P=0}=0 \\
\left.\frac{\partial^{2} L}{\partial P^{2}}\left(u_{1}(P), P\right)\right|_{P=0}=0
\end{array}\right.
$$

em $u_{1}^{1}, u_{1}^{2}$, obtemos que na carta de Monge

$$
u_{1}^{1}=0, \quad \text { e } \quad u_{1}^{2}=-\frac{B k-B+3 q_{111} q_{201}}{k-1},
$$

implicando que a solução $u_{1}=u_{1}(P)$ de $L\left(u_{1}, P\right)=0$ satisfaz

$$
u_{1}(P)=-\frac{B k-B+3 q_{111} q_{201}}{k-1} P^{2}+O(3) .
$$

Substituindo (3.38) em (3.31) obtemos que

$$
\begin{aligned}
u_{2}(P) & =\left(\frac{A}{2 c}-\frac{C}{2 c}+\frac{\left(-q_{201}+q_{021}\right) B}{2 q_{111} c}-\frac{q_{111}{ }^{2}}{(k-1) c}+\frac{-k^{3}}{c}+\frac{q_{201} q_{021}}{(k-1) c}\right) . \\
& \cdot\left(\frac{B k-B+3 q_{111} q_{201}}{k-1}\right)^{2} P^{4}+O(5) \\
u_{3}(P) & =\left(-\frac{B}{2 q_{111}}-\frac{q_{201}}{k-1}\right)\left(\frac{B k-B+3 q_{111} q_{201}}{k-1}\right)^{2} P^{4}+O(5),
\end{aligned}
$$

concluindo, assim, a demonstração do lema.

Os retratos de fase dos campos $X$ e $Y$, numa vizinhança das suas curvas de equilibrios, $\gamma$ e $\beta$, são descritos nos lemas 14, 15 e 16. 
Pontos Parcialmente Umbílicos $D_{1}^{1}, D_{2}^{1}, D_{3}^{1}, D_{13}^{1}, D_{1 h, p}^{1}$ e $D_{1 h, n}^{1}$

Lema 14 Restrito à hipersuperfície de Lie-Cartan, a curva de equilíbrio de $Y$ é normalmente hiperbólica do tipo sela.

Demonstração: Vimos na seção 1.2 do capítulo 1 , que na carta $\left(u_{1}, u_{2}, u_{3}, Q\right)$ a hipersuperfície de Lie-Cartan é dada por $\mathcal{G}\left(u_{1}, u_{2}, u_{3} ; Q\right)=0$, onde

$$
\mathcal{G}\left(u_{1}, u_{2}, u_{3} ; Q\right)=L_{r}\left(u_{1}, u_{2}, u_{3}\right)+M_{r}\left(u_{1}, u_{2}, u_{3}\right) Q+N_{r}\left(u_{1}, u_{2}, u_{3}\right) Q^{2} .
$$

Como, na carta de Monge reduzida, $\frac{\partial \mathcal{G}}{\partial u_{3}}(0,0,0,0)=-q_{111} \neq 0$, segue-se que podemos escrever $u_{3}=u_{3}\left(u_{1}, u_{2}, Q\right)$ em $\mathcal{G}\left(u_{1}, u_{2}, u_{3} ; Q\right)=0$. Tal função é dada por

$$
\begin{aligned}
u_{3}\left(u_{1}, u_{2}, Q\right) & =\left(-\frac{B}{2 q_{111}}-\frac{q_{201}}{k-1}\right) u_{1}^{2}+\left(-\frac{C-k^{3}}{q_{111}}-\frac{q_{201} q_{021}+q_{111}^{2}}{(k-1) q_{111}}\right) u_{1} u_{2} \\
& -\left(\frac{D}{2 q_{111}}+\frac{q_{021}}{(k-1) q_{111}}\right) u_{2}^{2}+\frac{c}{q_{111}} Q u_{2}+O(3) .
\end{aligned}
$$

Substituindo (3.39) em (3.33) teremos que, nas variáveis $u_{1}, u_{2}$ e $Q$, o campo vetorial de Lie-Cartan $Y_{\mathcal{G}}$ é dado por

$$
Y_{\mathcal{G}}=\left\{\begin{array}{rl}
\dot{u}_{1} & =Y_{1}\left(u_{1}, u_{2}, u_{3}\left(u_{1}, u_{3}, Q\right), Q\right) \\
\dot{u}_{2} & =Y_{2}\left(u_{1}, u_{2}, u_{3}\left(u_{1}, u_{3}, Q\right), Q\right) \\
\dot{Q} & =Y_{4}\left(u_{1}, u_{2}, u_{3}\left(u_{1}, u_{3}, Q\right), Q\right)
\end{array} .\right.
$$

onde

$$
\begin{aligned}
& Y_{1}=Q Y_{2} \\
& Y_{2}=c u_{2}+O(2) \\
& Y_{4}=\left(-k^{3}+C+\frac{2 q_{111}^{2}+q_{201} q_{021}}{k-1}\right) u_{1}+\left(D+\frac{3 q_{111} q_{021}}{k-1}\right) u_{2}-c Q+O(2)
\end{aligned}
$$

Pelo lema 13, os equilíbrios de $Y_{\mathcal{G}}$ formam uma curva regular $\gamma$ e esta pode ser escrita em função de $u_{1}$. A linearização de $Y_{\mathcal{G}}$, ao longo de $\gamma\left(u_{1}\right)$ possui os seguintes autovalores:

$$
\begin{aligned}
& \lambda_{1}\left(u_{1}\right) \equiv 0 \\
& \lambda_{2}\left(u_{1}\right)=c+O(1), \\
& \lambda_{3}\left(u_{1}\right)=-c+O(1)
\end{aligned}
$$

donde vemos que, restrito a hipersuperfície de Lie-Cartan, a curva de equilíbrios de $Y_{\mathcal{G}}$ é normalmente hiperbólica do tipo sela. 
Pontos Parcialmente Umbílicos $D_{1}^{1}, D_{2}^{1}, D_{3}^{1}, D_{13}^{1}, D_{1 h, p}^{1}$ e $D_{1 h, n}^{1}$

Nosso objetivo, agora, é estudar o campo de Lie-Cartan, $X$, restrito a hipersuperfície de Lie-Cartan, $\mathcal{L}\left(u_{1}, u_{2}, u_{3}, P\right)=0$, numa vizinhança da curva de equilíbrios $\beta$ descrita no lema 13. Para isto, observamos que

$$
\frac{\partial \mathcal{L}}{\partial u_{3}}(0,0,0,0)=q_{111} \neq 0
$$

implica que podemos escrever $u_{3}=u_{3}\left(u_{1}, u_{2}, P\right)$ em

$$
\mathcal{L}\left(u_{1}, u_{2}, u_{3}, P\right)=0
$$

Tal função, que define a hipersuperfície de Lie-Cartan como gráfico, é dada a seguir:

$$
\begin{aligned}
u_{3}\left(u_{1}, u_{2}, P\right) & =-\frac{1}{2} \frac{\left(B k+2 q_{111} q_{201}-B\right)}{q_{111}(k-1)} u_{1}^{2}-\frac{1}{2} \frac{\left(D k+2 q_{111} q_{021}-D\right)}{q_{111}(k-1)} u_{2}^{2}- \\
& -\frac{c}{q_{111}} P u_{2}-\frac{\left(C k-C-k^{4}+k^{3}+q_{201} q_{021}+q_{111}^{2}\right)}{q_{111}(k-1)} u_{1} u_{2}+O(3)
\end{aligned}
$$

Denotemos $X=\left(X_{1}, X_{2}, X_{3}, X_{4}\right)$. O campo de Lie-Cartan, restrito à hipersuperfície de Lie-Cartan, é dado por

$$
X_{\mathcal{L}}=\left\{\begin{array}{l}
\dot{u}_{1}=X_{1}\left(u_{1}, u_{2}, u_{3}\left(u_{1}, u_{2}, P\right), P\right) \\
\dot{u}_{2}=X_{2}\left(u_{1}, u_{2}, u_{3}\left(u_{1}, u_{2}, P\right), P\right) \\
\dot{P}=X_{4}\left(u_{1}, u_{2}, u_{3}\left(u_{1}, u_{2}, P\right), P\right)
\end{array}\right.
$$

onde

$$
\begin{aligned}
X_{1} & =c u_{2}+\left(\frac{A-C}{2}-k(k-1)^{2}+\frac{q_{201} q_{021}}{k-1}-\frac{\left(q_{201}+q_{021}\right) B}{q_{111}}+\frac{q_{111}{ }^{2}}{k-1}\right) u_{1}^{2}+ \\
& +\left(\frac{\left(-q_{021}+q_{201}\right) C}{q_{111}}+D-B-\frac{\left(-q_{021}+q_{201}\right) q_{111}}{k-1}+\frac{q_{201} q_{021}}{q_{111} q_{021}}-\right. \\
& \left.-\frac{q_{201} k^{3}}{q_{111}}-\frac{q_{201} q_{021}}{q_{111}(k-1)}+\frac{q_{021} k^{3}}{q_{111}}\right) u_{1} u_{2}+\left(-\frac{c\left(q_{021}-q_{201}\right)}{q_{111}}\right) u_{2} P+ \\
& +\left(-\frac{\left(-q_{201}+q_{021}\right) D}{2 q_{111}}-\frac{C+D}{2}-\frac{q_{111}^{2}}{k-1}-k^{3}+\frac{q_{201} q_{021}}{k-1}\right) u_{2}^{2}+O(3) \\
X_{2} & =X_{1} \cdot P
\end{aligned}
$$


Pontos Parcialmente Umbílicos $D_{1}^{1}, D_{2}^{1}, D_{3}^{1}, D_{13}^{1}, D_{1 h, p}^{1}$ e $D_{1 h, n}^{1}$

$$
\begin{aligned}
X_{4} & =\left(-C-\frac{2 q_{111}^{2}}{k-1}-\frac{q_{021} q_{201}}{k-1}+k^{3}\right) u_{2}-\frac{B k+3 q_{111} q_{201}-B}{k-1} u_{1}- \\
& -c P^{2}+\left(\left(\frac{Q_{211}}{2 q_{111}}+\frac{q_{201} q_{012}}{2(k-1) q_{111}}-\frac{q_{102}}{k-1}\right) B-\frac{q_{111} Q_{301}}{-2+2 k}\right) u_{1}^{2}+ \\
& +\left(\left(\frac{C}{(k-1) q_{111}}-\frac{q_{021} q_{201}}{(k-1) q_{111}}-\frac{q_{111}}{k-1}-\frac{k^{3}}{q_{111}}\right) Q_{211}-\frac{q_{012} q_{201} C}{(k-1) q_{111}}+\right. \\
& +\frac{2 q_{102} C}{k-1}+\frac{q_{201}{ }^{2} q_{012} q_{021}}{\left(k-1^{2} q_{111}\right.}+\frac{q_{201} q_{021} q_{102}}{(k-1)^{2}}-\frac{2 q_{111} q_{012} q_{201}}{(k-1)^{2}}-\frac{k^{3} q_{012} q_{201}}{(k-1) q_{111}}+ \\
& \left.+\frac{Q_{121} q_{201}}{k-1}+2 \frac{k^{3}(k+1) q_{102}}{(k-1)^{2}}\right) u_{2} u_{1}+\left(-2 C+A-\frac{4 q_{111}}{k-1}+\frac{3 q_{201}{ }^{2}}{k-1}-\right. \\
& \left.-2 \frac{q_{201} q_{021}}{k-1}-k^{3}\right) P u_{1}+\left(\frac{Q_{211} D}{2(k-1) q_{111}}+\frac{q_{021} q_{102} q_{111}}{(k-1)^{2}}+\frac{q_{201} q_{012} q_{021}}{(k-1)^{2}}\right. \\
& \left.+\frac{q_{102} D}{k-1}+\frac{q_{012} q_{201} D}{2(k-1) q_{111}}+\frac{q_{021} Q_{211}}{k-1}-2 \frac{q_{012} q_{111}^{2}}{(k-1)^{2}}-\frac{3 Q_{121} q_{111}}{2(k-1)}\right) u_{2}^{2}+ \\
& +\left(\frac{Q_{211} c}{q_{111}}+\frac{2 q_{102} c}{k-1}+\frac{q_{012} q_{201} c}{(k-1) q_{111}}-2 D+B+\frac{3 q_{201} q_{111}}{k-1}-\frac{6 q_{021} q_{111}}{k-1}\right) P u_{2} \\
& +O(3)
\end{aligned}
$$

Lema 15 A variedade

$$
W=\left\{\left(u_{1}, u_{2}, P\right) ; u_{2}=c_{2}\left(u_{1}\right)\right\}, \quad(\text { Veja figura } 3.8)
$$

onde $c_{2}\left(u_{1}\right)$ foi dada em (3.31), é invariante pelo fluxo de $X_{\mathcal{L}}$ e contém a curva de equilíbrios (numa vizinhança da origem). Além disso, o retrato de fase de $X_{\mathcal{L}}$ restrito à variedade $W$ é como na figura abaixo.

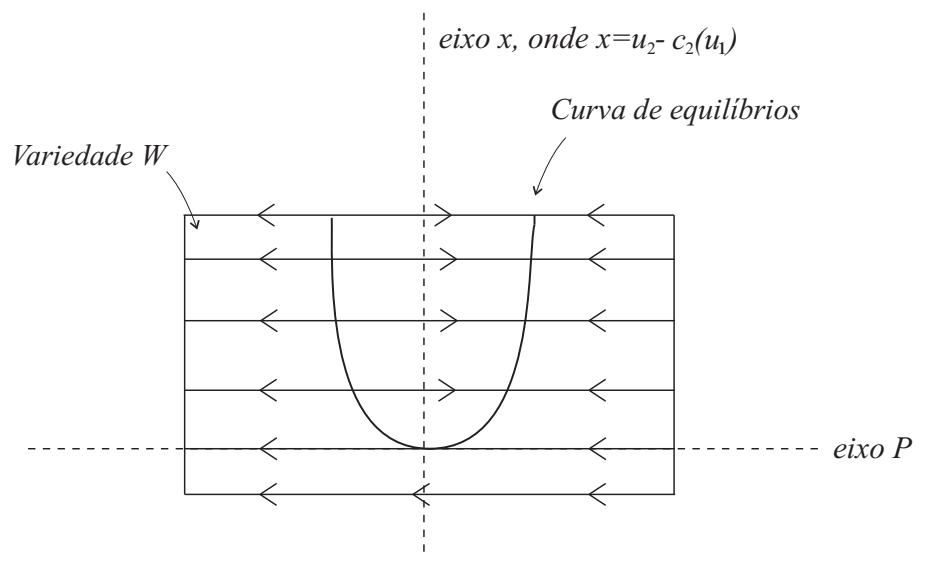

Figura 3.7: Retrato de fase de $X_{\mathcal{L}}$ restrito a variedade $W$. 
Pontos Parcialmente Umbílicos $D_{1}^{1}, D_{2}^{1}, D_{3}^{1}, D_{13}^{1}, D_{1 h, p}^{1}$ e $D_{1 h, n}^{1}$

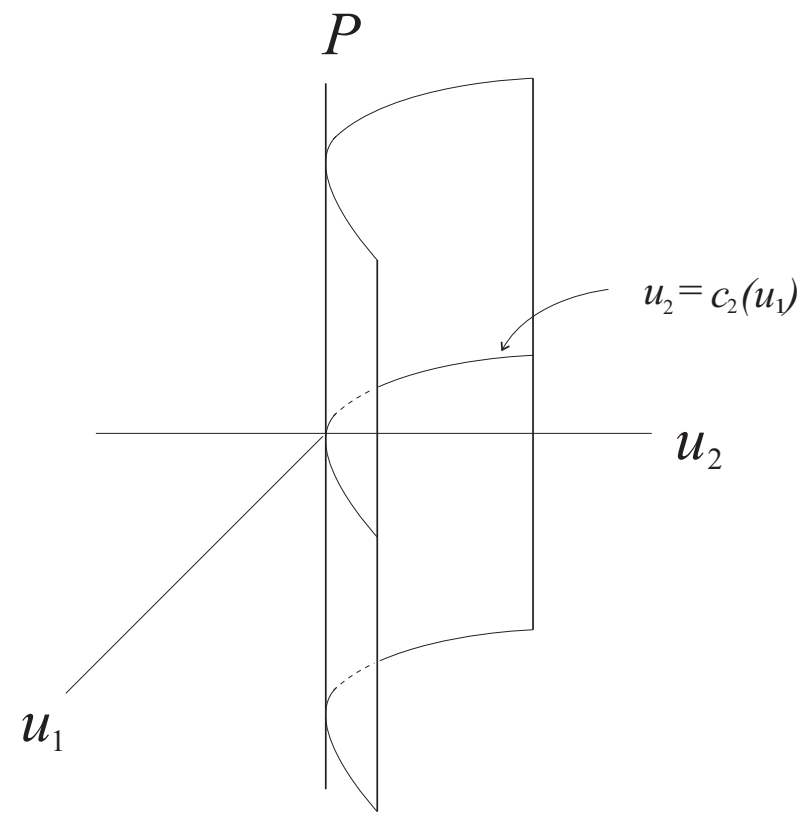

Figura 3.8: Variedade W.

Demonstração: Vimos no lema 13 que, nas variáveis $u_{1}, u_{2}$ e $P$, os equilíbrios de $X$ satisfazem

$$
u_{1}=u_{1}(P), \quad u_{2}=u_{2}(P), \text { onde } u_{2}(P)=c_{2}\left(u_{1}(P)\right) ;
$$

implicando que a curva de equilíbrios pertence a variedade $W$. Quanto a invariância de $W$ por $X_{\mathcal{L}}$, basta ver que $W$ contem o eixo $P$ e que

$$
\begin{aligned}
\dot{u}_{1} & =0 \\
\left(u_{1}, u_{2}, P\right) \in W \Rightarrow \quad \dot{u}_{2} & =0 \\
\dot{P} & =X_{4}\left(u_{1}, c_{2}\left(u_{1}\right), P\right)
\end{aligned}
$$

Por (3.43), vemos que o campo em $W$ é paralelo ao eixo $P$, e por (3.36) teremos que

$$
\frac{\partial^{2} X_{4}}{\partial P^{2}}(0,0,0)=-c \neq 0 \text { e } \frac{\partial X_{4}}{\partial u_{1}}(0,0,0)=-\frac{B k-B+3 q_{111} q_{201}}{k-1} \neq 0
$$

donde segue-se que o retrato de fase de $X_{\mathcal{L}}$ restrito a $W$ é como na figura 3.7. 
Pontos Parcialmente Umbílicos $D_{1}^{1}, D_{2}^{1}, D_{3}^{1}, D_{13}^{1}, D_{1 h, p}^{1}$ e $D_{1 h, n}^{1}$

Lema 16 Restrito à hipersuperfície de Lie-Cartan, o retrato de fase de $X$ numa vizinhança da curva de equilíbrios, $\beta$, é topologicamente como na figura 3.9.

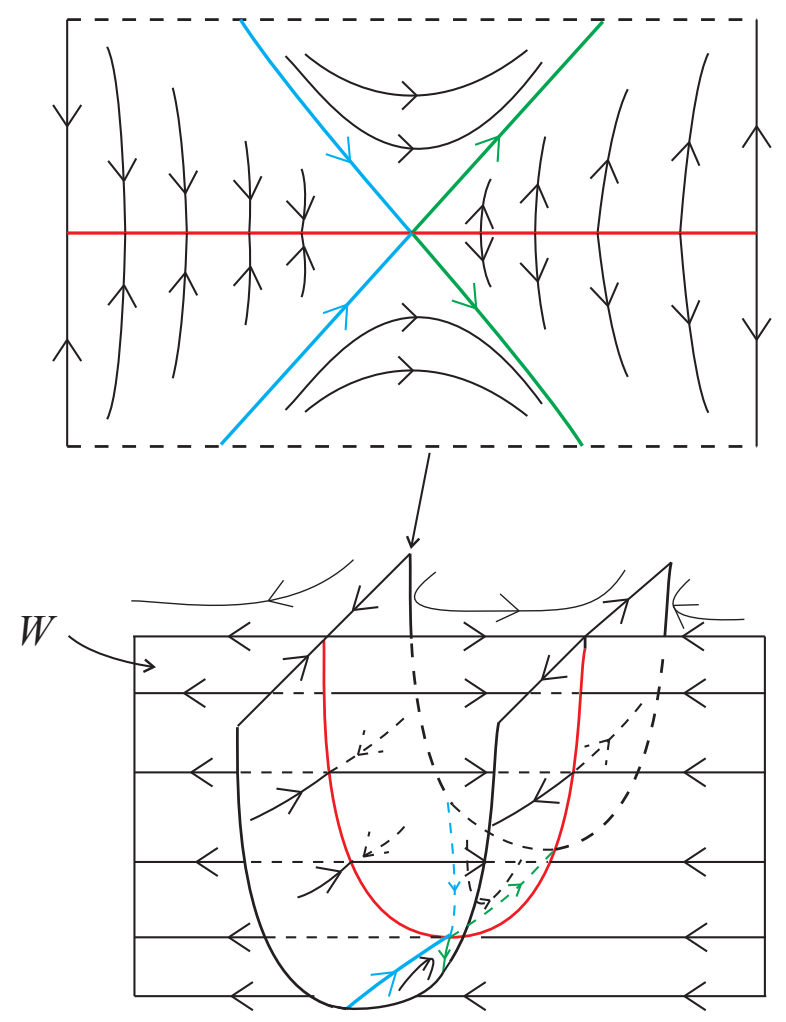

Figura 3.9: Nesta figura, a curva vermelha representa a curva de equilíbrios.

Demonstração: Escrevendo a curva de equilíbrios como no lema 13, teremos que os autovalores da derivada de $X_{\mathcal{L}}$, nos pontos desta curva, são dados em função de $P$ :

$$
\begin{aligned}
& \lambda_{1}(P) \equiv 0 \\
& \lambda_{2}(P)=-2 c P+O(2) \\
& \lambda_{3}(P)=c P+O(2)
\end{aligned}
$$

donde vemos que, fora de uma vizinhança da origem, a curva de equilíbrios de $X$ é normalmente hiperbólica do tipo sela.

Observação 21 O auto-vetor, da linearização de $X_{\mathcal{L}}$ na curva de equilíbrios, associado ao auto-valor $\lambda_{2}(P)$ é constante igual a $(0,0,1)$ (eixo $P$ ). 
Pontos Parcialmente Umbílicos $D_{1}^{1}, D_{2}^{1}, D_{3}^{1}, D_{13}^{1}, D_{1 h, p}^{1}$ e $D_{1 h, n}^{1}$

Se $P=0$ teremos três autovalores nulos (a variedade central na origem tem dimensão $3)$, e a forma de Jordan de $D X_{\mathcal{L}}(0,0,0)$ é

$$
\left(\begin{array}{lll}
0 & 1 & 0 \\
0 & 0 & 1 \\
0 & 0 & 0
\end{array}\right) .
$$

Para o estudo de $X_{\mathcal{L}}$ num vizinhança da origem, faremos a seguinte mudança de coordenadas:

$$
u_{1}=r^{2} x, \quad u_{2}=r^{3} y, \quad P=r z, \quad x^{2}+y^{2}+z^{2}=1, \quad r \geq 0 .
$$

Das relações acima, teremos que

$$
\left\{\begin{array}{l}
\dot{u}_{1}=2 r \dot{r} x+r^{2} \dot{x} \\
\dot{u}_{2}=3 r \dot{r}^{2} y+r^{3} \dot{y} \\
\dot{P}=\dot{r} z+r \dot{z} \\
0=\dot{x} x+\dot{y} y+\dot{z} z
\end{array}\right.
$$

Resolvendo em $\dot{r}, \dot{x}, \dot{y}$ e $\dot{z}$, usando (3.44) e (3.42), e multiplicando pela função positiva $\frac{\left(2 x^{2}+3 y^{2}+z^{2}\right)}{r(k-1)}$ obtemos o seguinte campo:

$$
\tilde{X}=\left\{\begin{aligned}
\dot{x} & =\frac{\left(2 k B+6 q_{201} q_{111}-2 B\right)}{k-1} z x^{2}-2 c z y^{2} x+2 c z^{3} x+3 c y^{3}+c z^{2} y+ \\
& +r F_{1}(x, y, z, r) \\
\dot{y} & =2 c z y x^{2}-3 c y^{2} x+\frac{\left(9 q_{201} q_{111}+3 k B-3 B\right)}{k-1} z y x+4 c z^{3} y+ \\
& +r F_{2}(x, y, z, r) \\
\dot{z} & =-\frac{\left(2 k B-2 B+6 q_{201} q_{111}\right)}{k-1} x^{3}-2 c z^{2} x^{2}-c z y x-4 c z^{2} y^{2}- \\
& -\frac{\left(9 q_{201} q_{111}+3 k B-3 B\right)}{k-1} y^{2} x+r F_{3}(x, y, z, r) \\
\dot{r} & =c y x-\frac{\left(k B-B+3 q_{111} q_{201}\right) z x}{k-1}+c z y^{2}-c z^{3}+r^{2} F_{4}(x, y, z, r)
\end{aligned}\right.
$$

onde $F_{i}(x, y, z, 0)=0, i=1,2,3,4$. Estudaremos a restrição deste campo à esfera 
Pontos Parcialmente Umbílicos $D_{1}^{1}, D_{2}^{1}, D_{3}^{1}, D_{13}^{1}, D_{1 h, p}^{1}$ e $D_{1 h, n}^{1}$

unitária $x^{2}+y^{2}+z^{2}=1$, ou seja, quando $r=0$. Neste caso, os equilíbrios são

- $(0,0, z)$ onde $z^{2}=1$,

- $\left(-\frac{(k-1) c}{k B-B+3 q_{201} q_{111}} z^{2}, 0, z\right)$ onde $\left(\frac{(k-1) c}{k B-B+3 q_{201} q_{111}} z^{2}\right)^{2}+z^{2}=1$,

- $\left(-\frac{4}{3} \frac{4 c(k-1) z^{2}}{3 q_{201} q_{111}+k B-B},-\frac{8}{9} \frac{c(k-1) z^{3}}{3 q_{201} q_{111}+k B-B}, z\right)$ onde

$\left(\frac{4}{3} \frac{4 c(k-1) z^{2}}{3 q_{201} q_{111}+k B-B}\right)^{2}+\left(\frac{8}{9} \frac{c(k-1) z^{3}}{3 q_{201} q_{111}+k B-B}\right)^{2}+z^{2}=1$.

Pode-se mostrar que são seis as singularidades na esfera.

Quanto a linearização do campo $\tilde{X}_{0}=\tilde{X}(x, y, z, 0)$, nas singularidades dadas acima, teremos que:

- Os autovalores não nulos de $D \tilde{X}_{0}(0,0, z)$ são $2 c z^{3}, 4 c z^{3}$

- Os autovalores não nulos de $D \tilde{X}_{0}\left(-\frac{(k-1) c}{k B-B+3 q_{201} q_{111}} z^{2}, 0, z\right)$ são

$2 c z^{3}\left(2 \frac{c^{2}(k-1)^{2} z^{2}}{\left(3 q_{201} q_{111}+(k-1) B\right)^{2}}+1\right),-2 c z^{3}\left(2 \frac{c^{2}(k-1)^{2} z^{2}}{\left(3 q_{201} q_{111}+(k-1) B\right)^{2}}+1\right)$

- Os autovalores não nulos de

$D \tilde{X}_{0}\left(-\frac{16 c(k-1) z^{2}}{9 q_{201} q_{111}+9(k-1) B},-\frac{8 c(k-1) z^{3}}{27 q_{201} q_{111}+9(k-1) B}, z\right)$ são

$-\frac{4}{81} c z^{3}\left(\frac{64(c(k-1))^{4} z^{4}}{\left(3 q_{201} q_{111}+(k-1) B\right)^{2}}+\frac{96(c(k-1))^{4} z^{2}}{\left(3 q_{201} q_{111}+(k-1) B\right)^{2}}+27\right) \mathrm{e}$

$-\frac{2}{27} c z^{3}\left(\frac{64(c(k-1))^{4} z^{4}}{\left(3 q_{201} q_{111}+(k-1) B\right)^{2}}+\frac{96(c(k-1))^{4} z^{2}}{\left(3 q_{201} q_{111}+(k-1) B\right)^{2}}+27\right)$

donde vemos que temos dois atratores, dois repulsores e duas selas.

Seja

$$
\mathcal{Q}(x, y, z)=c y z+\frac{(k-1) B+3 q_{201} q_{111}}{2 k-2} x^{2} .
$$

Denotemos as componentes de $\tilde{X}_{0}$ por $\dot{x}=\tilde{X}_{0}^{1}, \dot{y}=\tilde{X}_{0}^{2}, \dot{z}=\tilde{X}_{0}^{3}, \dot{r}=\tilde{X}_{0}^{4}$. Como

$$
c \tilde{X}_{0}^{2} z+y \tilde{X}_{0}^{3}+\frac{(k-1) B+3 q_{201} q_{111}}{k-1} x \tilde{X}_{0}^{1}=0
$$

se $c y z+\frac{(k-1) B+3 q_{201} q_{111}}{2 k-2} x^{2}=0$, segue-se que o cone quadrático $\mathcal{Q}^{-1}(0)$ é invariante por $\tilde{X}_{0}$. Além disso,

$$
\mathcal{Q}(0,0, z)=0, \mathcal{Q}\left(-\frac{4}{3} \frac{4 c(k-1) z^{2}}{3 q_{201} q_{111}+k B-B},-\frac{8}{9} \frac{c(k-1) z^{3}}{3 q_{201} q_{111}+k B-B}, z\right)=0
$$


Pontos Parcialmente Umbílicos $D_{1}^{1}, D_{2}^{1}, D_{3}^{1}, D_{13}^{1}, D_{1 h, p}^{1}$ e $D_{1 h, n}^{1}$

$\mathrm{e}$

$$
\mathcal{Q}\left(-\frac{(k-1) c}{k B-B+3 q_{201} q_{111}} z^{2}, 0, z\right)=\frac{1}{2} \frac{c^{2} z^{4}(k-1)}{3 q_{201} q_{111}+k B-B} \neq 0
$$

ou seja, apenas os atratores e os repulsores pertencem à interseção deste cone com a esfera.

Numa vizinhança da esfera, as quatro singularidades

$$
(0,0, z, 0)
$$

e

$$
\left(-\frac{16 c(k-1) z^{2}}{9 q_{201} q_{111}+9(k-1) B},-\frac{8 c(k-1) z^{3}}{27 q_{201} q_{111}+9(k-1) B}, z\right)
$$

são selas hiperbólicas para $X$. De fato, além dos autovalores citados acima (da restrição a esfera) teremos que

$$
-c z^{3} \text { e } \frac{1}{81} c z^{3}\left(\frac{64(c(k-1))^{4} z^{4}}{\left(3 q_{201} q_{111}+(k-1) B\right)^{2}}+\frac{96(c(k-1))^{4} z^{2}}{\left(3 q_{201} q_{111}+(k-1) B\right)^{2}}+27\right) .
$$

são autovalores associados às singularidades (3.45) e (3.46), respectivamente.

Veja ilustração nas figuras 3.10 e 3.11, do retrato de fase de $X$ próximo a esfera.

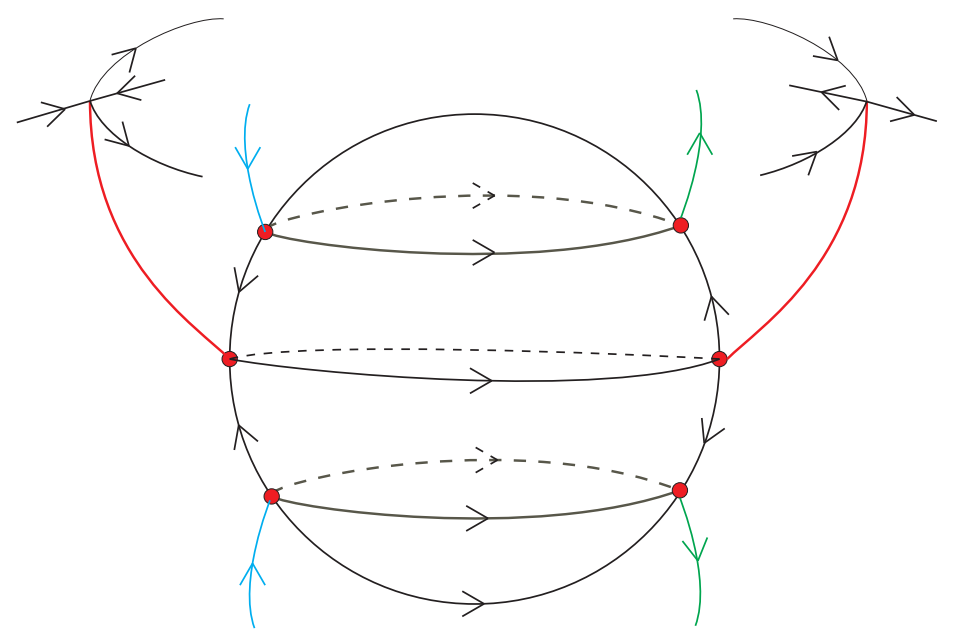

Figura 3.10: Retrato de fase de $\tilde{X}$ próximo a esfera. 
Pontos Parcialmente Umbílicos $D_{1}^{1}, D_{2}^{1}, D_{3}^{1}, D_{13}^{1}, D_{1 h, p}^{1}$ e $D_{1 h, n}^{1}$

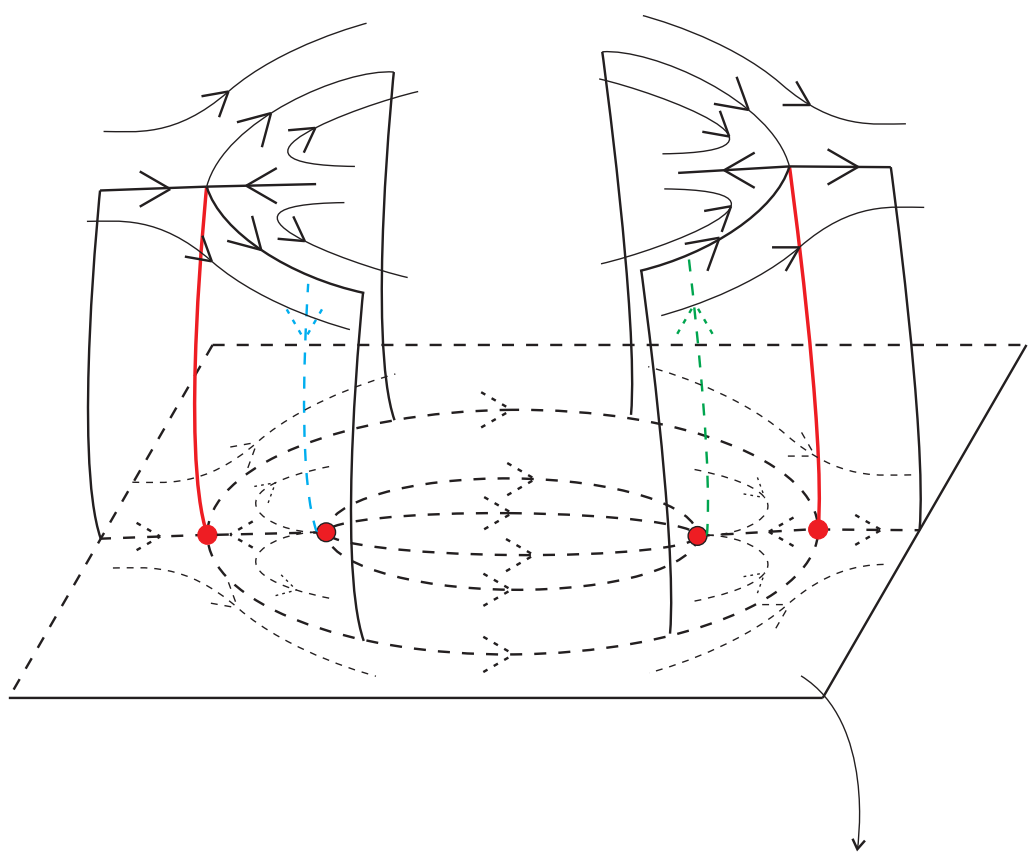

Vista superior da esfera

Figura 3.11: Retrato de fase de $X$ numa vizinhança da esfera, parte superior.

Assim, próximo a origem, o retrato de fase de $X_{\mathcal{L}}$ é como na figura 3.9. 
Pontos Parcialmente Umbílicos $D_{1}^{1}, D_{2}^{1}, D_{3}^{1}, D_{13}^{1}, D_{1 h, p}^{1}$ e $D_{1 h, n}^{1}$

Demonstração do teorema 7: Seja $p$ um ponto parcialmente umbílicos do tipo $D_{2 h}^{1}$. Por (3.30), $p$ pertence a uma curva regular $c$ formada por pontos parcialmente umbílicos.

Sejam $X$ o campo de lie-Cartan na carta $\left(u_{1}, u_{2}, u_{3}, P\right)$ (veja (3.32)) e $Y$ o campo de lie-Cartan na carta $\left(u_{1}, u_{2}, u_{3}, Q\right)$ (veja (3.33)).

Pelo lema 13, o campo $Y$ possui uma única curva de equilíbrios, $\gamma$, passando transversalmente por $Q=0$ e o campo $X$, possui uma única curva de equilíbrios, $\beta$, numa vizinhança da origem, satisfazendo

$$
\begin{aligned}
u_{1}(P) & =\frac{(k-1) c}{B(k-1)+3 q_{111} q_{201}} P^{2}+O(3) \\
u_{2}(P) & =\left(\frac{A}{2 c}-\frac{C}{2 c}+\frac{\left(-q_{201}+q_{021}\right) B}{2 q_{111} c}-\frac{q_{111}{ }^{2}}{(k-1) c}+\frac{-k^{3}}{c}+\frac{q_{201} q_{021}}{(k-1) c}\right) . \\
& \cdot\left(\frac{B k-B+3 q_{111} q_{201}}{k-1}\right)^{2} P^{4}+O(5) \\
u_{3}(P) & =\left(-\frac{B}{2 q_{111}}-\frac{q_{201}}{k-1}\right)\left(\frac{B k-B+3 q_{111} q_{201}}{k-1}\right)^{2} P^{4}+O(5), .
\end{aligned}
$$

Pelo lema 14, $\gamma$ é normalmente hiperbólica do tipo sela, e

Pelos lemas 15 e 16 o retrato de fase de $X$ restrito a hipesuperfície de Lie-Cartan, numa vizinhança de $\beta$, é como na figura 3.9. (veja figura 3.12) 


\section{Lema 6}

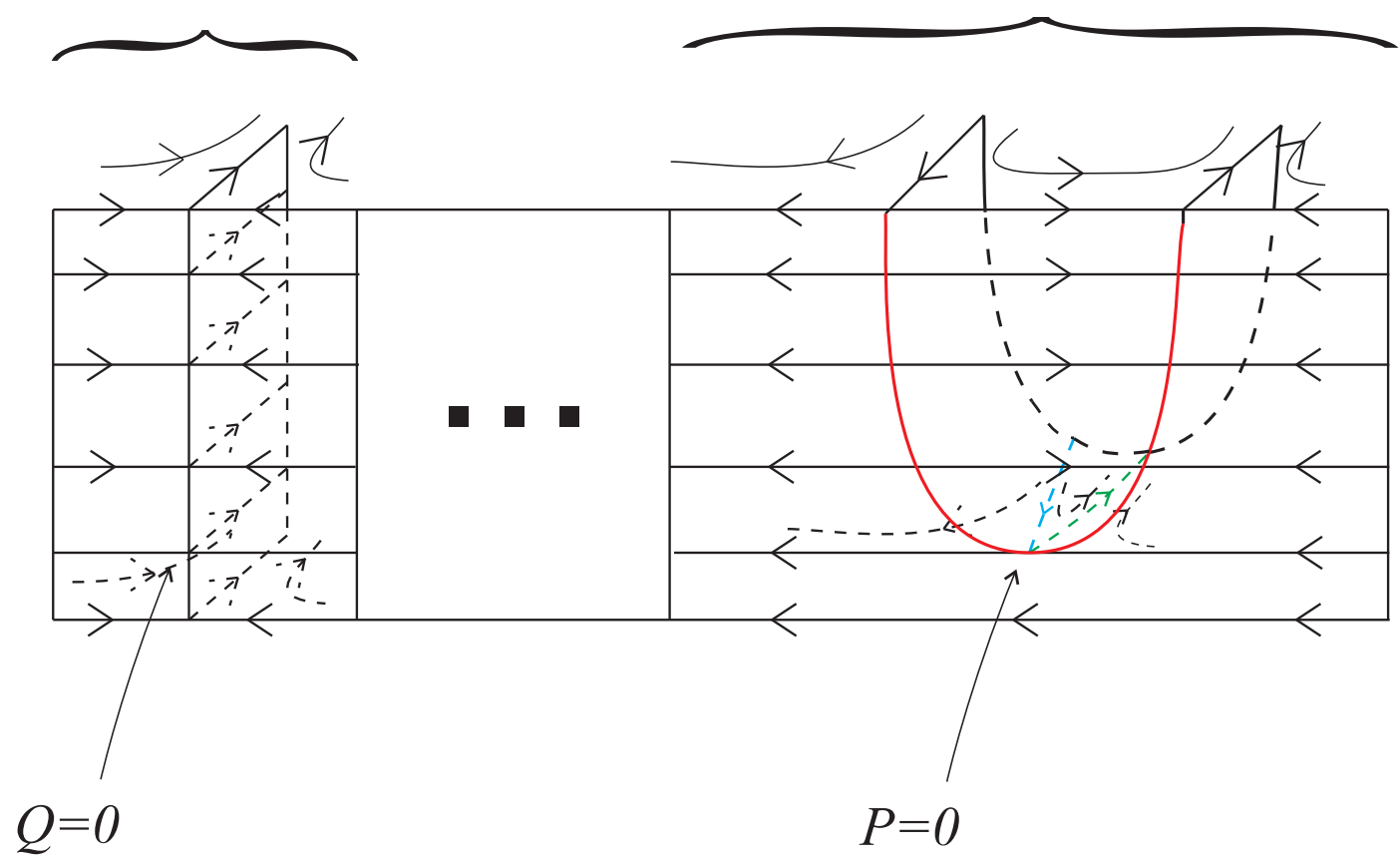

Figura 3.12: Ilustração dos resultados obtidos nesta seção.

Assim, concluímos que a curva parcialmente umbílica $c$ é, por uma lado, a projeção de três curvas do tipo sela e por outro a de uma curva do tipo sela, implicando que, em $p$, a estrutura da curva parcialmente umbílica muda do tipo $D_{1}$ para o tipo $D_{3}$, concluindo, assim, a prova do teorema 7 .

Observação 22 O caso $c=b=0$ nos leva a mesma estrutura tológica da linhas de curvatura numa vizinahnça de um ponto do tipo $D_{13}^{1}$. 
Pontos Parcialmente Umbílicos $D_{1}^{1}, D_{2}^{1}, D_{3}^{1}, D_{13}^{1}, D_{1 h, p}^{1}$ e $D_{1 h, n}^{1}$

\subsection{Linhas de Curvatura numa Vizinhança de um Ponto $D_{1 h, p}^{1}$ e $D_{1 h, n}^{1}$}

Seja

$$
X:=\left\{\begin{array}{l}
\dot{u}_{1}=\mathcal{L}_{P} \\
\dot{u}_{2}=P \mathcal{L}_{P} \\
\dot{u}_{3}=(\mathcal{U}+\mathcal{V} P) \mathcal{L}_{P} \\
\dot{P}=-\left(\mathcal{L}_{u_{1}}+P \mathcal{L}_{u_{2}}+\mathcal{L}_{u_{3}}(\mathcal{U}+\mathcal{V} P)\right)
\end{array},\right.
$$

onde $\mathcal{L}=L_{r} P^{2}+M_{r} P+N_{r}$.

Os equilíbrios de $X$ são dados por

$$
\left\{\begin{array}{l}
\left\{\begin{array}{l}
L_{r}\left(u_{1}, u_{2}, u_{3}\right)=0 \\
M_{r}\left(u_{1}, u_{2}, u_{3}\right)=0
\end{array}\right. \\
\left(\mathcal{L}_{u_{1}}+P \mathcal{L}_{u_{2}}+\mathcal{L}_{u_{3}}(\mathcal{U}+\mathcal{V} P)\right)=0
\end{array}\right.
$$

Suponhamos que

$$
\operatorname{det}\left(\left.\frac{\partial\left(L_{r}, M_{r}\right)}{\partial\left(u_{1}, u_{2}\right)}\right|_{u_{1}=0, u_{2}=0, u_{3}=0}\right) \neq 0
$$

Na carta de Monge reduzida, a não anulação do determinante acima é equivalente: $b(b-a) \neq 0$ (veja a equação 1.15 na proposição 1 do capítulo 1 )

Seja

$$
u_{1}=u_{1}\left(u_{3}\right) \text { e } u_{2}=u_{2}\left(u_{3}\right)
$$

a solução de

$$
\left\{\begin{array}{l}
L_{r}\left(u_{1}, u_{2}, u_{3}\right)=0, \\
M_{r}\left(u_{1}, u_{2}, u_{3}\right)=0,
\end{array}\right.
$$

substituindo (3.49) na última equação de (3.48), obtemos que os equilíbrios de $X$ são dados pela anulação da seguinte cúbica

$$
C\left(u_{3}, P\right)=A_{3}\left(u_{3}\right) P^{3}+A_{2}\left(u_{3}\right) P^{2}+A_{1}\left(u_{3}\right) P+A_{0}\left(u_{3}\right),
$$


Pontos Parcialmente Umbílicos $D_{1}^{1}, D_{2}^{1}, D_{3}^{1}, D_{13}^{1}, D_{1 h, p}^{1}$ e $D_{1 h, n}^{1}$

onde

$$
\begin{aligned}
& A_{3}=-\frac{\partial}{\partial u_{2}} L_{r}-\frac{\partial}{\partial u_{3}} L_{r} \mathcal{U} \\
& A_{2}=-\frac{\partial}{\partial u_{1}} L_{r}-\frac{\partial}{\partial u_{2}} M_{r}-\left(\frac{\partial}{\partial u_{3}} M_{r}\right) \mathcal{U}-\left(\frac{\partial}{\partial u_{3}} L_{r}\right) \mathcal{V} \\
& A_{1}=-\frac{\partial}{\partial u_{1}} M_{r}-\frac{\partial}{\partial u_{2}} N_{r}-\left(\frac{\partial}{\partial u_{3}} N_{r}\right) \mathcal{U}-\left(\frac{\partial}{\partial u_{3}} M_{r}\right) \mathcal{V}, \\
& A_{0}=-\frac{\partial}{\partial u_{1}} N_{r}-\left(\frac{\partial}{\partial u_{3}} N_{r}\right) \mathcal{V} .
\end{aligned}
$$

Denotemos por $D\left(u_{3}\right)$ o discriminante de (3.50). Sejam

$$
\chi_{2}=\frac{\partial D}{\partial u_{3}}(0)(\text { Veja equações } 2.32 \text { e } 3.26 \text { no capítulo } 2)
$$

e

$$
\tilde{\chi}_{2}=\frac{\partial^{2} D}{\partial u_{3}^{2}}(0)
$$

Definição 11 (Ponto parcialmente umbílico $D_{1 h, p}^{1}$ e $D_{1 h, n}^{1}$ ) Seja

$$
\alpha\left(u_{1}, u_{2}, u_{3}\right)=\left(u_{1}, u_{2}, u_{3}, h\left(u_{1}, u_{2}, u_{3}\right)\right),
$$

com h como em (3.2). O ponto $p=\alpha(0,0,0)$ é chamado parcialmente umbílico do tipo:

$\left.D_{1 h, p}^{1}\right) c b(b-a) \neq 0, \frac{a}{b}=2, \chi_{2}=0$ e $\tilde{\chi}_{2}>0$.

$\left.D_{1 h, n}^{1}\right) c b(b-a) \neq 0, \frac{a}{b}=2, \chi_{2}=0$ e $\tilde{\chi}_{2}<0$.

Observação 23 Se fizermos $q_{111}=0, q_{201}=0, q_{021}=0, c=1, b=1, a=2$, $Q_{121}=0, Q_{211}=0, B=0, C=0, Q_{112}=0$ e $Q_{301}=0$, obtemos que $c b(b-a) \neq 0$, $\frac{a}{b}=2, \chi_{2}=0$ e $\tilde{\chi}_{2}=\frac{4}{27} q_{102}^{2} q_{012} \neq 0$. 
Pontos Parcialmente Umbílicos $D_{1}^{1}, D_{2}^{1}, D_{3}^{1}, D_{13}^{1}, D_{1 h, p}^{1}$ e $D_{1 h, n}^{1}$

Teorema 8 Suponhamos $\alpha \in \mathcal{I}^{k}\left(\mathbb{M}^{3}, \mathbb{R}^{4}\right), k \geq 5$ e seja $p$ um ponto parcialmente umbílico do tipo $D_{1 h, n}^{1}$. Então o comportamento das linhas de curvatura próximas à $p$ é como na figura 3.13.

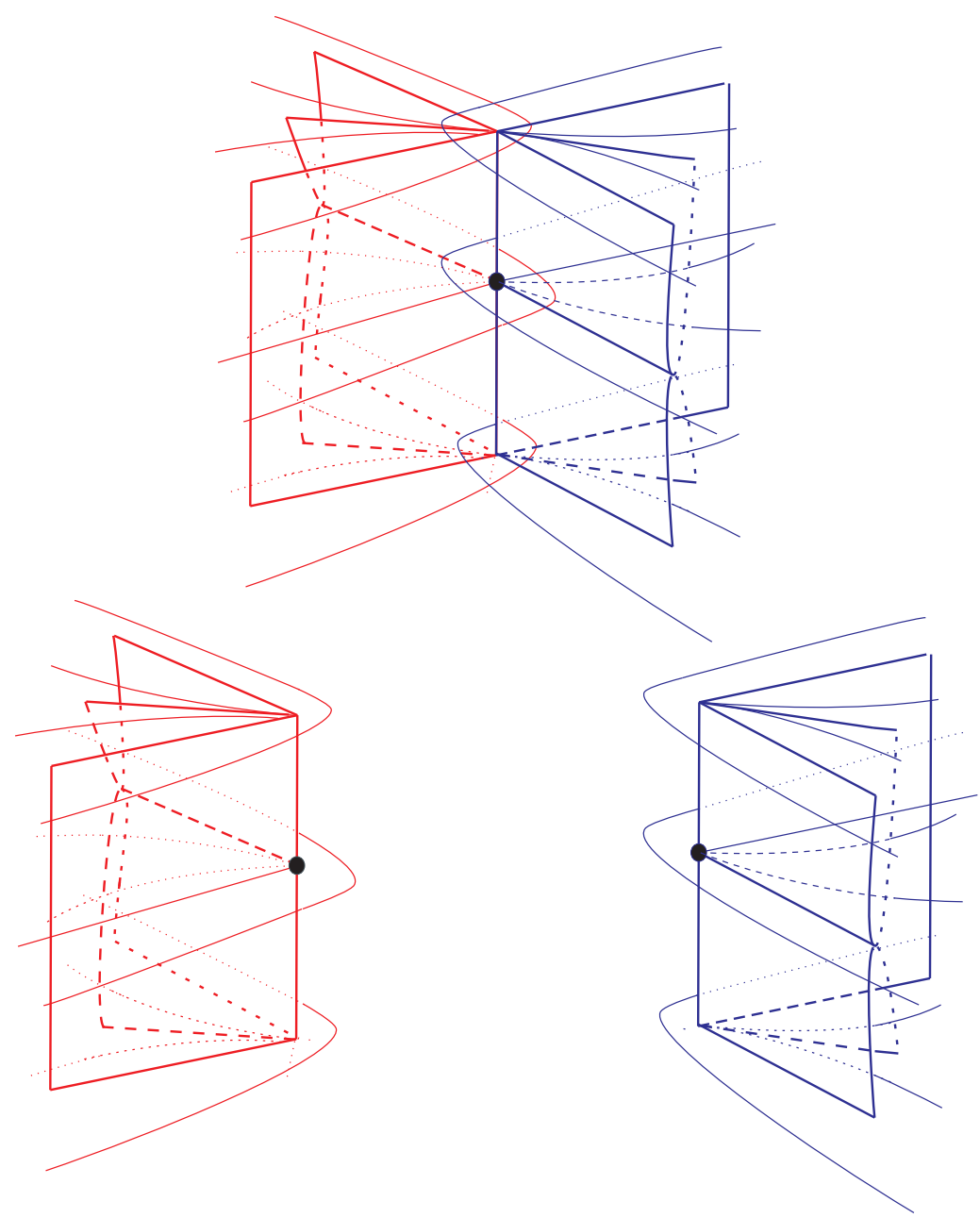

Figura 3.13: Linhas de curvatura numa vizinhança de um ponto do tipo $D_{1 h, n}^{1}$. 
Pontos Parcialmente Umbílicos $D_{1}^{1}, D_{2}^{1}, D_{3}^{1}, D_{13}^{1}, D_{1 h, p}^{1}$ e $D_{1 h, n}^{1}$

Teorema 9 Suponhamos $\alpha \in \mathcal{I}^{k}\left(\mathbb{M}^{3}, \mathbb{R}^{4}\right), k \geq 5$ e seja $p$ um ponto parcialmente umbílico do tipo $D_{1 h, p}^{1}$. Então o comportamento das linhas de curvatura próximas à $p$ é como na figura 3.14.

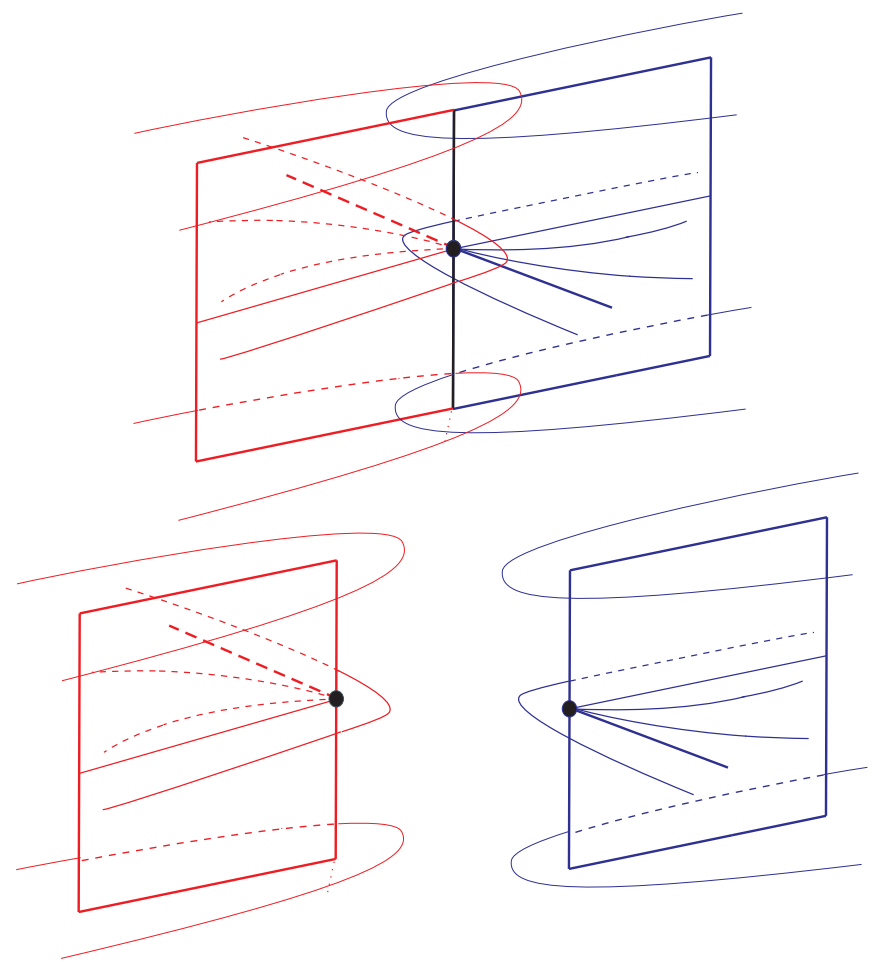

Figura 3.14: Linhas de curvatura numa vizinhança de um ponto do tipo $D_{1 h, p}^{1}$.

Demonstração dos teoremas 8 e 9: Os equilíbrios de $X$ no eixo $P$ são dados por $C(0, P)=0$ (equação 3.50). Na carta de Monge

$$
C(0, P)=0 \Leftrightarrow b P^{3}-c P^{2}=0 \Leftrightarrow P=0 \text { ou } P=\frac{c}{b} .
$$

Sendo $\left.\frac{\partial C}{\partial P}\right|_{u_{3}=0, P=\frac{c}{b}}=\frac{c^{2}}{b} \neq 0$ segue-se que podemos escrever $P=P\left(u_{3}\right)$ na equação $C\left(u_{3}, P\right)=0$. Seja $\beta_{1}$ a curva cujas coordenadas satisfazem:

$$
\beta_{1}: u_{1}=c_{1}\left(u_{3}\right), \quad u_{2}=c_{2}\left(u_{3}\right) \text { e } P=P\left(u_{3}\right)
$$

onde $u_{1}=c_{1}\left(u_{3}\right)$ e $u_{2}=c_{2}\left(u_{3}\right)$ são as coordenadas da curva parcialmente umbílica. A linearização, $D X\left(\beta_{1}\left(u_{3}\right)\right)$, do campo de Lie-Cartan possui dois autovalores nulos e os seguintes dois não nulos:

$$
\lambda_{1}\left(u_{3}\right)=-\frac{b^{2}+c^{2}}{b}+O(1), \quad \lambda_{2}\left(u_{3}\right)=\frac{c^{2}}{b}+O(1) .
$$


Pontos Parcialmente Umbílicos $D_{1}^{1}, D_{2}^{1}, D_{3}^{1}, D_{13}^{1}, D_{1 h, p}^{1}$ e $D_{1 h, n}^{1}$

Disto, restrito à hipersuperfície de Lie-Cartan, $\beta_{1}$ é normalmente hiperbólica do tipo sela.

Seja $\varepsilon>0$ suficientemente pequeno. Definamos

$$
W=\left\{\left(u_{1}, u_{2}, u_{3}, P\right), \quad\left|u_{3}\right|<\varepsilon, \quad u_{1}=c_{1}\left(u_{3}\right) \text { e } u_{2}=c_{2}\left(u_{3}\right)\right\} .
$$

Então,

1. $W$ é uma variedade diferenciável de classe $C^{k-2}$ e invariante pelo fluxo de $X$.

2. $W$ está contida na hipersuperfície de Lie-Cartan

3. As coordendas de um ponto de $W$ satisfaz:

$$
\dot{u}_{1}=0, \dot{u}_{2}=0, \dot{u}_{3}=0
$$

e

$$
\dot{P}=C\left(u_{3}, P\right)
$$

O discriminante, $D\left(u_{3}\right)$, da equação cúbica $C\left(u_{3}, P\right)$ satifaz

$$
\frac{\partial D}{\partial u_{3}}(0,0)=\chi_{2}=0 \text { e } \frac{\partial^{2} D}{\partial u_{3}^{2}}(0,0)=b \tilde{\chi}_{2} \neq 0
$$

Denotemos por $X_{\mathcal{L}}$ o campo de Lie-Cartan restrito à hipersuperfície de LieCartan.

O retrato de fase de $X_{\mathcal{L}}$ restrito a variedade $W$ é como na figura 3.15 se $\tilde{\chi}_{2}<0$ e como na figura 3.16 se $\tilde{\chi}_{2}>0$ 
Pontos Parcialmente Umbílicos $D_{1}^{1}, D_{2}^{1}, D_{3}^{1}, D_{13}^{1}, D_{1 h, p}^{1}$ e $D_{1 h, n}^{1}$

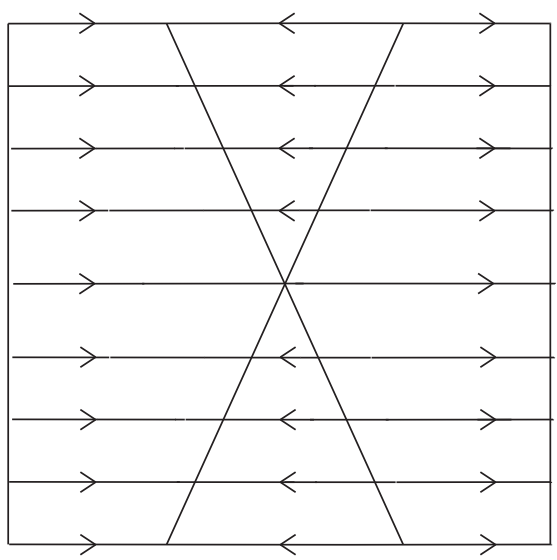

Figura 3.15: Comportamento de $X_{\mathcal{L}}$ restrito a variedade central quando $\tilde{\chi}_{2}<0$

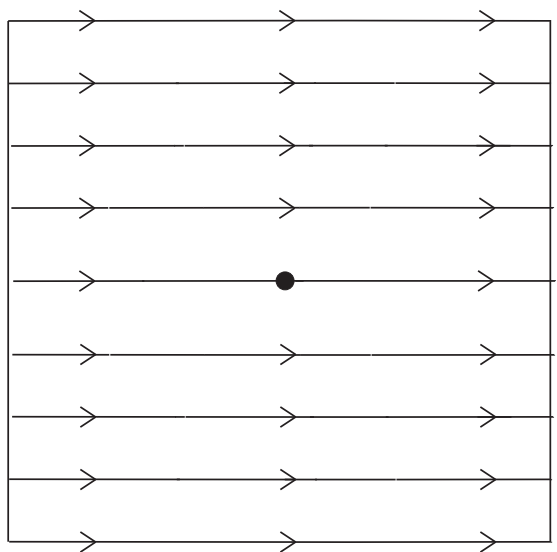

Figura 3.16: Comportamento de $X_{\mathcal{L}}$ restrito a variedade central quando $\tilde{\chi}_{2}>0$

A linearização de $X$, na origem, possui uma autovalor não nulo: $-b$. Assim, o retrato de fase de $X_{\mathcal{L}}$ numa vizinhança da origem é como na figura 3.17 se $\tilde{\chi}_{2}<0$ e como na figura 3.18 se $\tilde{\chi}_{2}>0$

Projetando as curvas integrais de $X$, numa vizinhança dos seus equilíbrios, na variedade $\mathbb{M}^{3}$ concluímos a demonstração dos teoremas 8 e 9 . 


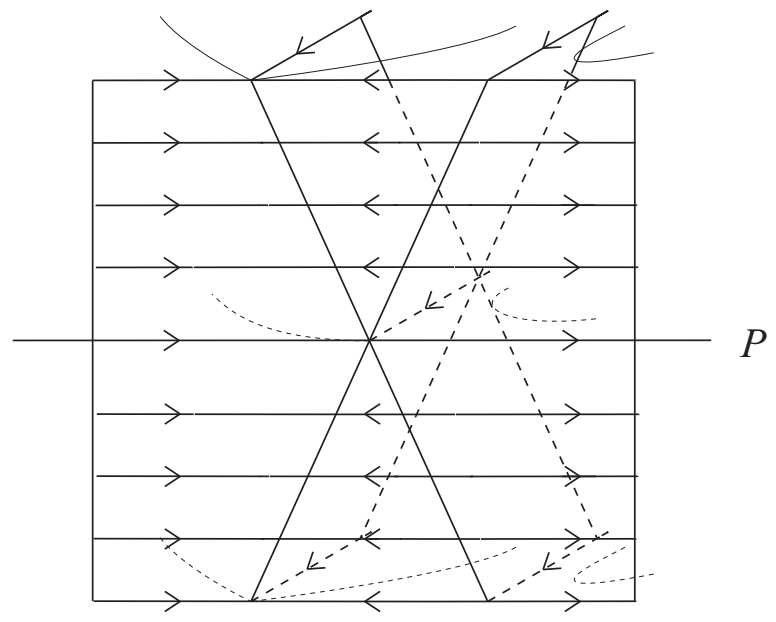

Figura 3.17: Comportamento de $X_{\mathcal{L}}$ numa vizinhança da origem quando $\tilde{\chi}_{2}<0$

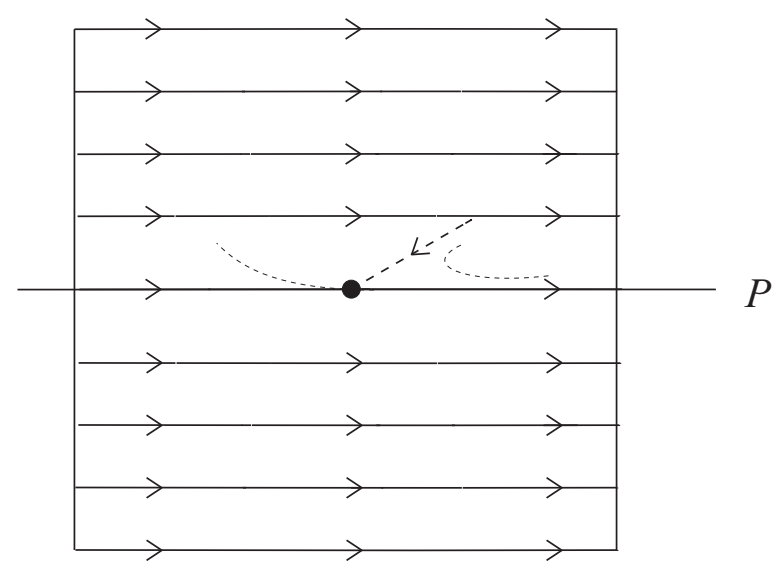

Figura 3.18: Comportamento de $X_{\mathcal{L}}$ numa vizinhança da origem quando $\tilde{\chi}_{2}>0$ 


\section{Capítulo 4}

\section{Pontos Parcialmente Umbílicos}

\section{Críticos: $D_{p}^{1}$ e $D_{c}^{1}$}

\subsection{Preliminares}

Seja $\alpha: \mathbb{M}^{3} \rightarrow \mathbb{R}^{4}$ e $\left(u_{1}, u_{2}, u_{3}\right):\left(\mathbb{M}^{3}, p\right) \rightarrow\left(\mathbb{R}^{3}, 0\right)$ a carta de Monge reduzida, ou seja,

$$
\alpha\left(u_{1}, u_{2}, u_{3}\right)=\left(u_{1}, u_{2}, u_{3}, h\left(u_{1}, u_{2}, u_{3}\right)\right)
$$

onde

$$
\begin{aligned}
h\left(u_{1}, u_{2}, u_{3}\right) & =\frac{k}{2}\left(u_{1}^{2}+u_{2}^{2}\right)+\frac{k_{3}}{2} u_{3}^{2}+\frac{1}{6} a u_{1}^{3}+\frac{1}{2} b u_{1} u_{2}^{2}+\frac{1}{6} c u_{2}^{3}+\frac{1}{6} q_{003} u_{3}^{3} \\
& +\frac{1}{2} q_{012} u_{2} u_{3}^{2}+q_{111} u_{1} u_{2} u_{3}+\frac{1}{2} q_{021} u_{2}^{2} u_{3}+\frac{1}{2} q_{102} u_{1} u_{3}^{2} \\
& +\frac{1}{2} q_{201} u_{1}^{2} u_{3}+\frac{1}{24} A u_{1}^{4}+\frac{1}{6} B u_{1}^{3} u_{2}+\frac{1}{4} C u_{1}^{2} u_{2}^{2}+\frac{1}{6} D u_{1} u_{2}^{3} \\
& +\frac{1}{24} E u_{2}^{4}+\frac{1}{24} Q_{004} u_{3}^{4}+\frac{1}{6} Q_{013} u_{2} u_{3}^{3}+\frac{1}{6} Q_{103} u_{1} u_{3}^{3}+\frac{1}{4} Q_{022} u_{2}^{2} u_{3}^{2} \\
& +\frac{1}{4} Q_{202} u_{1}^{2} u_{3}^{2}+\frac{1}{2} Q_{112} u_{1} u_{2} u_{3}^{2}+\frac{1}{6} Q_{031} u_{2}^{3} u_{3}+\frac{1}{6} Q_{301} u_{1}^{3} u_{3} \\
& +\frac{1}{2} Q_{121} u_{1} u_{2}^{2} u_{3}+\frac{1}{2} Q_{211} u_{1}^{2} u_{2} u_{3}+O(5) .
\end{aligned}
$$

Lembramos que o conjunto parcialmente umbílico de $\alpha$ é definido por:

$$
\left\{\begin{array}{l}
L_{r}\left(u_{1}, u_{2}, u_{3}\right)=0 \\
M_{r}\left(u_{1}, u_{2}, u_{3}\right)=0
\end{array} .\right.
$$


Pontos Parcialmente Umbílicos $D_{p}^{1}$ e $D_{c}^{1}$

onde

$$
\begin{aligned}
L_{r} & =-b u_{2}-q_{111} u_{3}-\frac{\left(2 q_{201} q_{111}+B k-B\right)}{2(k-1)} u_{1}^{2}-\left(\frac{\left(2 q_{201} q_{021}+2 q_{111}{ }^{2}\right)}{2(k-1)}+\right. \\
& \left.+C-2 k^{3}+\right) u_{2} u_{1}-\left(Q_{211}+\frac{\left(2 q_{102} q_{111}+2 q_{201} q_{012}\right)}{2(k-1)}\right) u_{3} u_{1}-\left(\frac{\left(2 q_{111} q_{021}\right)}{2(k-1)}+\right. \\
& \left.+\frac{D}{2}\right) u_{2}^{2}-\left(Q_{121}+\frac{\left(q_{102} q_{021}+q_{111} q_{012}\right)}{(k-1)}\right) u_{2} u_{3}-\left(\frac{\left(q_{012} q_{102}\right)}{(k-1)}+\right. \\
& \left.+\frac{Q_{112}}{2}\right) u_{3}{ }^{2}+O(3)
\end{aligned}
$$

$$
\begin{aligned}
M_{r} & =(b-a) u_{1}+c u_{2}-\left(q_{201}-q_{021}\right) u_{3}+\left(-\frac{A}{2}+\frac{C}{2}+k^{3}-\frac{q_{201}^{2}-q_{111}^{2}}{k-1}\right) u_{1}^{2}+ \\
& +\left(-B-D-\frac{2 q_{201} q_{111}-2 q_{111} q_{021}}{k-1}\right) u_{1} u_{2}+\left(-\frac{-2 q_{111} q_{012}+2 q_{201} q_{102}}{k-1}+\right. \\
& \left.+Q_{121}-Q_{301}\right) u_{1} u_{3}+\left(\frac{E}{2}-\frac{C}{2}-k^{3}-\frac{q_{111}^{2}-q_{021}^{2}}{k-1}\right) u_{2}^{2}+\left(-Q_{211}+Q_{031}-\right. \\
& \left.-\frac{-2 q_{021} q_{012}+2 q_{102} q_{111}}{k-1}\right) u_{2} u_{3}+\left(\frac{Q_{022}}{2}-\frac{Q_{202}}{2}-\frac{-q_{012}^{2}+q_{102}^{2}}{k-1}\right) u_{3}^{2}+O(3)
\end{aligned}
$$

$$
\begin{aligned}
N_{r} & =b u_{2}+q_{111} u_{3}+\frac{\left(2 q_{201} q_{111}+B k-B\right)}{2(k-1)} u_{1}^{2}+\left(\frac{\left(2 q_{201} q_{021}+2 q_{111}^{2}\right)}{2(k-1)}+\right. \\
& \left.+C-2 k^{3}+\right) u_{2} u_{1}+\left(Q_{211}+\frac{\left(2 q_{102} q_{111}+2 q_{201} q_{012}\right)}{2(k-1)}\right) u_{3} u_{1}+\left(\frac{\left(2 q_{111} q_{021}\right)}{2(k-1)}+\right. \\
& \left.+\frac{D}{2}\right) u_{2}^{2}+\left(Q_{121}+\frac{\left(q_{102} q_{021}+q_{111} q_{012}\right)}{(k-1)}\right) u_{2} u_{3}+\left(\frac{\left(q_{012} q_{102}\right)}{(k-1)}+\right. \\
& \left.+\frac{Q_{112}}{2}\right) u_{3}^{2}+O(3)
\end{aligned}
$$


donde vemos que

$$
\left.\frac{\partial\left(L_{r}, M_{r}\right)}{\partial\left(u_{1}, u_{2}, u_{3}\right)}\right|_{u_{1}=0, u_{2}=0, u_{3}=0}=\left(\begin{array}{ccc}
0 & -b & -q_{111} \\
-a+b & c & -q_{201}+q_{021}
\end{array}\right) .
$$

Diremos que o conjunto parcialmente umbílico da $\alpha$ é regular se a matriz (4.5) tem posto 2, caso contrário, diremos que o conjunto parcialmente umbílico da $\alpha$ é crítico.

Observamos que (4.5) deixa de ter posto 2, da maneira mais simples possível, se ocorrer um dos seguintes dois casos:

Caso 1: $b=0$ e $q_{111}=0$, ou

Caso 2: $a=b$ e $b\left(q_{201}-q_{021}\right)+c q_{111}=0$

Observação 24 Iremos supor $k=0$.

Analisaremos o primeiro caso. O segundo caso é análogo.

Supondo $a \neq 0$, podemos escrever $u_{1}=u_{1}\left(u_{2}, u_{3}\right)$ em $M_{r}\left(u_{1}, u_{2}, u_{3}\right)=0$, numa vizinhança da origem, já que $\frac{\partial M_{r}}{\partial u_{1}}(0,0,0)=-a \neq 0$. Na carta de Monge reduzida, tal função é dada por:

$$
\begin{aligned}
u_{1}\left(u_{2}, u_{3}\right)= & \frac{c}{a} u_{2}+\frac{\left(-q_{201}+q_{021}\right)}{a} u_{3}+ \\
& +\frac{1}{2 a^{3}}\left[2(-B+D) a c+\left(-C+E-2 q_{021}^{2}\right) a^{2}+\left(C+2 q_{201}^{2}-A\right) c^{2}\right] u_{2}^{2}+ \\
& +\frac{1}{a^{3}}\left[2 c q_{201} q_{102} a-2 q_{201} q_{012} a^{2}-Q_{301} c a+Q_{121} c a-Q_{211} a^{2}+Q_{031} a^{2}-\right. \\
& \left.-\left(-q_{201}+q_{021}\right)\left(A c-C c+a B-a D-2 q_{201}^{2} c\right)\right] u_{2} u_{3}- \\
& -\frac{1}{2 a^{3}}\left[Q_{022} a^{2}-2 q_{012}^{2} a^{2}+2 q_{102}^{2} a^{2}-Q_{202} a^{2}-\right. \\
& -\left(-q_{201}+q_{021}\right)\left(\left(-q_{201}+q_{021}\right)\left(-2 q_{201}^{2}+A-C+4 a q_{102}\right)-\right. \\
& \left.\left.-8 q_{021} q_{102} a-4 Q_{121} a+4 Q_{301} a\right)\right] u_{3}^{2}+O(3)
\end{aligned}
$$


Substituindo (4.6) em (4.2) e multiplicando por $a^{2}$, obtemos que

$$
\begin{aligned}
L_{r}\left(u_{1}\left(u_{2}, u_{3}\right), u_{2}, u_{3}\right) & =\left(D a^{2}+B c^{2}-2 q_{201} q_{021} a c+2 C a c\right) u_{2}^{2}+ \\
& +\left(\left(2 q_{021}-2 q_{201}\right)\left(-q_{021} q_{201} a+C a+B c+a q_{012} c\right)+\right. \\
& \left.+2 a\left(-a q_{102} q_{021}+c Q_{211}+a Q_{121}-q_{021} q_{012} c\right)\right) u_{2} u_{3}+ \\
& +\left(\left(q_{021}-q_{201}\right)\left(B q_{021}+2 a Q_{211}-q_{201} B-2 a q_{201} q_{012}\right)-\right. \\
& \left.-a^{2}\left(-2 q_{012} q_{102}+Q_{112}\right)\right) u_{3}^{2}+O(3)
\end{aligned}
$$

CONDIÇÃO DE QUASE TRANSVERSALIDADE: Determinante da matriz hessiana de $L_{r}\left(u_{1}\left(u_{2}, u_{3}\right), u_{2}, u_{3}\right)$, na origem, diferente de zero. Denotemos por $\zeta$ este determinante.

Na carta de Monge reduzida com $k_{3}=1$ e $k=0$ teremos que

$$
\begin{aligned}
\zeta & =4\left(-q_{201}+q_{021}\right)^{2}\left(-q_{201}^{2} q_{021}^{2}+2 C q_{201} q_{021}+D B-C^{2}\right)+ \\
& -8 a\left(-q_{201}+q_{021}\right)\left(q_{201} q_{102} q_{021}^{2}-q_{021} C q_{102}-Q_{121} q_{201} q_{021}+C Q_{121}-Q_{211} D+\right. \\
& \left.+D q_{201} q_{012}\right)-8 c\left(-q_{201}+q_{021}\right)\left(-q_{021} B q_{102}+q_{021} Q_{211} q_{201}-q_{021} q_{201}^{2} q_{012}+B Q_{121}+\right. \\
& \left.+C q_{201}+q_{012}-C Q_{211}\right)+\left(8 q_{201} q_{012} Q_{211}+4 B Q_{112}-4 q_{201}^{2} q_{012}^{2}-8 B q_{102} q_{012}+\right. \\
& \left.+8 Q_{121} q_{102} q_{021}-4 Q_{211}^{2}\right) c^{2}+\left(4 D Q_{112}-4 q_{102}^{2} q_{021}^{2}-4 Q_{121}^{2}-8 D q_{102} q_{012}-4 q_{102}^{2} q_{021}^{2}-\right. \\
& \left.-4 Q_{121}^{2}+8 Q_{121} q_{102} q_{021}-8 D q_{102} q_{012}+4 D Q_{112}\right) a^{2}+\left(-8 Q_{211} Q_{121}+8 Q_{211} q_{102} q_{021}+\right. \\
& \left.+8 C Q_{112}-16 C q_{102} q_{012}-8 q_{201} q_{021} Q_{112}+8 q_{201} q_{012} Q_{121}+8 q_{201} q_{012} q_{102} q_{021}\right) a c
\end{aligned}
$$

Observação 25 - Se $\zeta>0$ então o conjunto solução da equação $L_{r}\left(u_{1}\left(u_{2}, u_{3}\right), u_{2}, u_{3}\right)=$ 0 é formado por um ponto.

- Se $\zeta<0$ então o conjunto solução da equação $L_{r}\left(u_{1}\left(u_{2}, u_{3}\right), u_{2}, u_{3}\right)=0$ é formado por duas curvas regulares que se intersectam transversalmente.

Disto, introduzimos as seguintes definições: 
Pontos Parcialmente Umbílicos $D_{p}^{1}$ e $D_{c}^{1}$

Definição 12 [Ponto Parcialmente Umbílico $D_{p}^{1}$ ] O ponto p é chamado parcialmente umbilico do tipo $D_{p}^{1}$ se:

$\left.D_{p}^{1}\right) b=q_{111}=0$ e $\zeta>0$.

Definição 13 [Ponto Parcialmente Umbílico $D_{c}^{1}$ J O ponto p é chamado parcialmente umbílico do tipo $D_{c}^{1}$ se:

$\left.D_{c}^{1}\right) b=q_{111}=0$ e $\zeta<0$.

Assim,

\section{Proposição 2}

Se $p$ é um ponto parcialmente umbílico do tipo $D_{p}^{1}$ então o conjunto parcialmente umbílico da $\alpha$, numa vizinhança de $p$, é formado por único ponto

\section{Proposição 3}

Se p é um ponto parcialmente umbílico do tipo $D_{c}^{1}$ então o conjunto parcialmente umbílico da $\alpha$, numa vizinhança de p, é formado por duas curvas regulares que se intersectam transversalmente em $p$. 
Pontos Parcialmente Umbílicos $D_{p}^{1}$ e $D_{c}^{1}$

\subsection{Linhas de curvatura numa vizinhança de um ponto parcialmente umbílico do tipo $D_{p}^{1}$ e $D_{c}^{1}$}

Nosso objetivo é provar os seguintes dois teoremas:

Teorema 10 Se p é um ponto parcialmente umbilico do tipo $D_{p}^{1}$ então o comportamento das linhas de curvatura de $\alpha$, numa vizinhança de p, é como na figura 4.1.
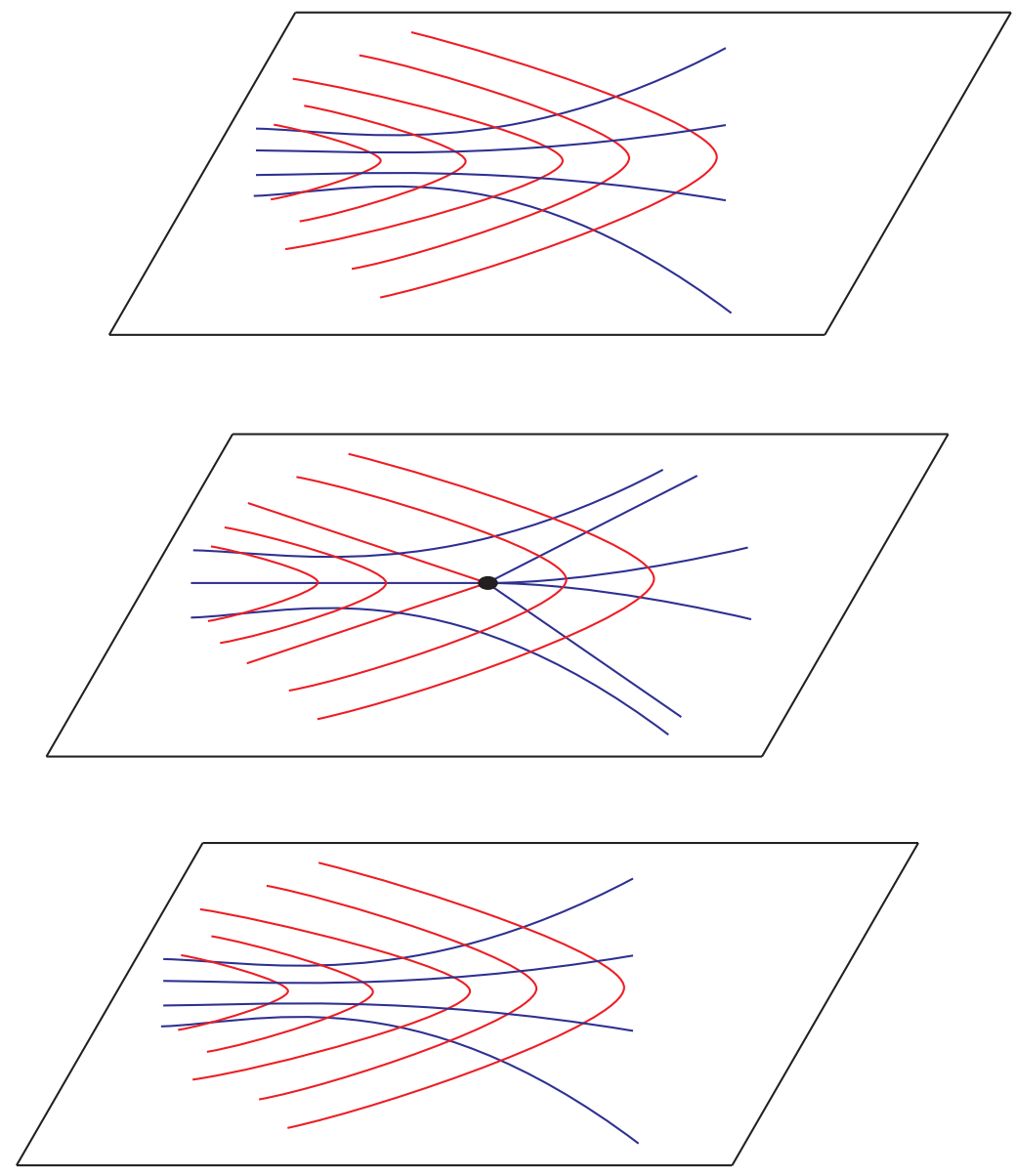

Figura 4.1: Configuração principal numa vizinhança de um ponto parcialmente umbílico do tipo $D_{p}^{1}$ 
Pontos Parcialmente Umbílicos $D_{p}^{1}$ e $D_{c}^{1}$

Teorema 11 Se p é um ponto parcialmente umbílico do tipo $D_{c}^{1}$ então o comportamento das linhas de curvatura de $\alpha$, numa vizinhança de p, é como na figura 4.2.

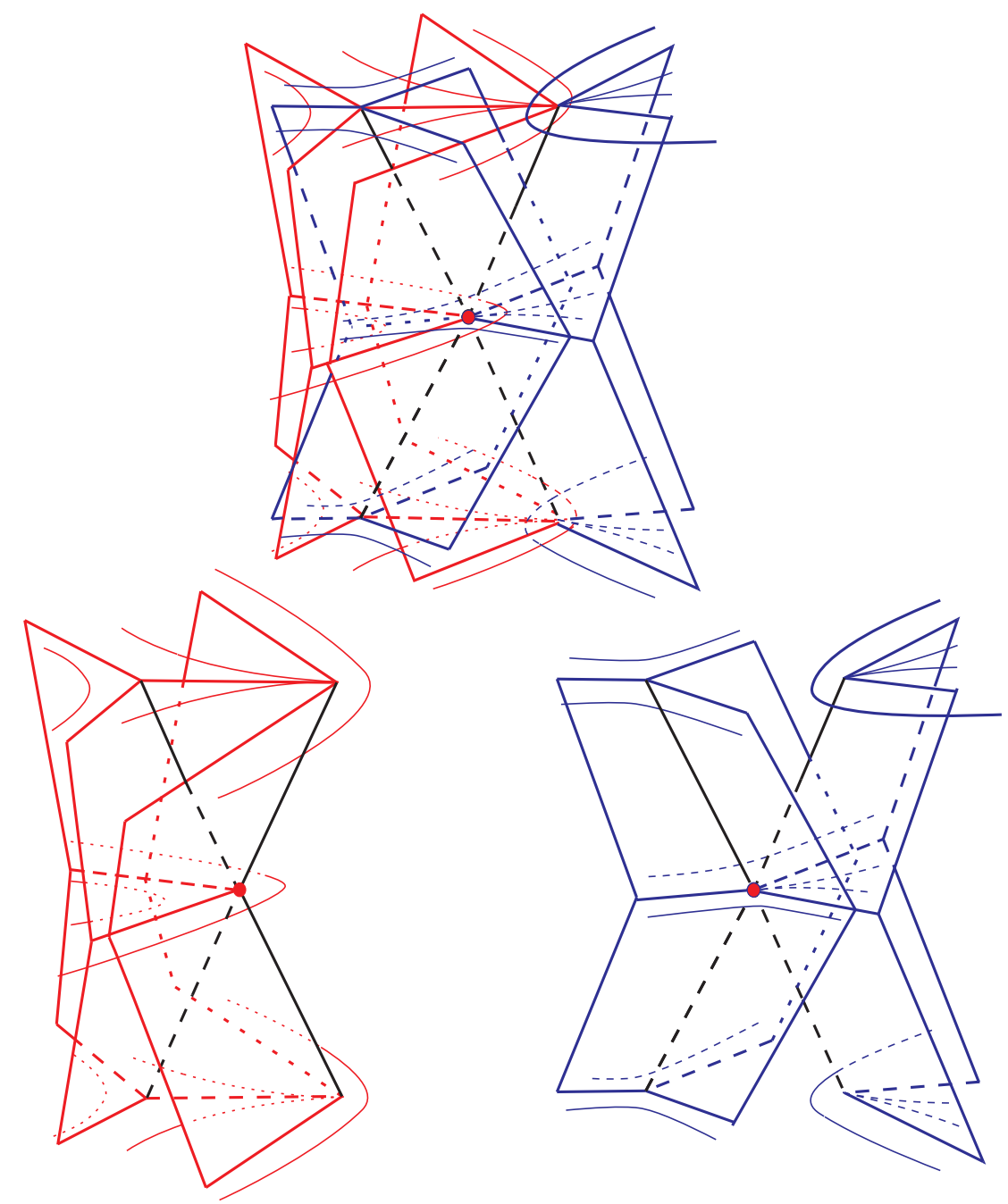

Figura 4.2: Configuração principal numa vizinhança de um ponto parcialmente umbílico do tipo $D_{c}^{1}$ 
Demonstração dos teoremas 10 e 11:

Lembramos que depois de feita a restrição das formas fundamentais ao campo de planos ortogonais a direção regular (seção 1.2 do capitulo 1), a equação diferencial das linhas de curvatura é dada por:

$$
\begin{aligned}
& L_{r}\left(u_{1}, u_{2}, u_{3}\right) d u_{1}^{2}+M_{r}\left(u_{1}, u_{2}, u_{3}\right) d u_{1} d u_{2}+N_{r}\left(u_{1}, u_{2}, u_{3}\right) d u_{2}^{2}=0 \\
& \text { Sejam } P=\frac{d u_{2}}{d u_{1}}, Q=\frac{d u_{1}}{d u_{2}}, \\
& \mathcal{L}\left(u_{1}, u_{2}, u_{3}, P\right)=L_{r}\left(u_{1}, u_{2}, u_{3}\right) P^{2}+M_{r}\left(u_{1}, u_{2}, u_{3}\right) P+N_{r}\left(u_{1}, u_{2}, u_{3}\right), \\
& \mathcal{G}\left(u_{1}, u_{2}, u_{3}, Q\right)=L_{r}\left(u_{1}, u_{2}, u_{3}\right)+M_{r}\left(u_{1}, u_{2}, u_{3}\right) Q+N_{r}\left(u_{1}, u_{2}, u_{3}\right) Q^{2}, \\
& X:=\left\{\begin{array}{l}
\dot{u}_{1}=\mathcal{L}_{P} \\
\dot{u}_{2}=P \mathcal{L}_{P} \\
\dot{u}_{3}=(\mathcal{U}+\mathcal{V} P) \mathcal{L}_{P} \\
\dot{P}=-\left(\mathcal{L}_{u_{1}}+P \mathcal{L}_{u_{2}}+\mathcal{L}_{u_{3}}(\mathcal{U}+\mathcal{V} P)\right)
\end{array}\right.
\end{aligned}
$$

$\mathrm{e}$

$$
Y:=\left\{\begin{aligned}
\dot{u}_{1} & =Q \frac{\partial \mathcal{G}}{\partial Q} \\
\dot{u}_{2} & =\frac{\partial \mathcal{G}}{\partial Q} \\
\dot{u}_{3} & =(\mathcal{U} Q+\mathcal{V}) \frac{\partial \mathcal{G}}{\partial Q} \\
\dot{Q} & =-\left(Q \mathcal{G}_{u_{1}}+\mathcal{G}_{u_{2}}+\mathcal{G}_{u_{3}}(\mathcal{U} Q+\mathcal{V})\right)
\end{aligned}\right.
$$

Observação 26 A condição de quase transversalidade, do conjunto parcialmente umbílico, implica que as hipersuperfícies implícitas $\mathcal{L}=0$ e $\mathcal{G}=0$ possuem, na origem, um ponto crítico do tipo Morse. De fato:

$$
\nabla \mathcal{L}(0,0,0,0)=\nabla \mathcal{G}(0,0,0,0)=(0,0,0,0)
$$

$e$

$$
\operatorname{det}(\text { Hessiana } \mathcal{L})=\operatorname{det}(\text { Hessiana } \mathcal{G})=-4 \zeta
$$

Na carta de Monge reduzida, o campo vetorial de Lie-Cartan $X$ é: 


$$
X=\left\{\begin{aligned}
\dot{u}_{1} & =c u_{2}+\left(-q_{201}+q_{021}\right) u_{3}-a u_{1}+\left(q_{201}^{2}-\frac{A}{2}+\frac{C}{2}\right) u_{1}^{2}+ \\
& \left(2 q_{201} q_{102}-Q_{301}+Q_{121}\right) u_{1} u_{3}+\left(-q_{021}^{2}+\frac{E}{2}-\frac{C}{2}\right) u_{2}^{2}+ \\
& +\left(-Q_{211}+Q_{031}-2 q_{021} q_{012}\right) u_{2} u_{3}+(-B+D) u_{1} u_{2}+ \\
& +\left(q_{102}^{2}-\frac{Q_{202}}{2}+\frac{Q_{022}}{2}-q_{012}^{2}\right) u_{3}^{2}+O(3) \\
\dot{u}_{2} & =\dot{u}_{1} P \\
\dot{u}_{3} & =\dot{u}_{1}\left(-q_{201} u_{1}-q_{102} u_{3}+O(2)\right) \\
\dot{P} & =\left(q_{201} q_{021}-C\right) u_{2}+\left(q_{201} q_{012}-Q_{211}\right) u_{3}-B u_{1}+a P+ \\
& +\left(-3 q_{201} q_{102}+Q_{301}-2 Q_{121}+2 q_{102} q_{021}\right) P u_{3}+(B-2 D) P u_{2}+ \\
& +\left(-2 q_{102}^{2} q_{012}+q_{102} Q_{112}\right) u_{3}^{2}+\left(Q_{121} q_{201}-q_{102} q_{021} q_{201}\right) u_{1} u_{2}-c P^{2}+ \\
& +\left(-q_{201}^{2} q_{012}+Q_{211} q_{201}\right) u_{1}^{2}+\left(q_{201} Q_{112}-3 q_{201} q_{012} q_{102}+q_{102} Q_{211}\right) u_{1} u_{3}+ \\
& +\left(-q_{102}^{2} q_{021}+q_{102} Q_{121}\right) u_{2} u_{3}+\left(-3 q_{201}^{2}+2 q_{201} q_{021}+A-2 C\right) P u_{1}+O(3)
\end{aligned}\right.
$$

e o campo $Y$ é:

$$
Y=\left\{\begin{aligned}
\dot{u}_{1} & =Q \dot{u}_{2} \\
\dot{u}_{2} & =c u_{2}+\left(q_{021}-q_{201}\right) u_{3}-a u_{1}+\left(q_{201}^{2}-\frac{A}{2}+\frac{C}{2}\right) u_{1}^{2}+(-B+D) u_{1} u_{2}+ \\
& +\left(2 q_{201} q_{102}-Q_{301}+Q_{121}\right) u_{1} u_{3}+\left(-q_{021}^{2}+\frac{E}{2}-\frac{C}{2}\right) u_{2}^{2}+ \\
& +\left(-Q_{211}+Q_{031}-2 q_{021} q_{012}\right) u_{2} u_{3}+\left(q_{102}^{2}-\frac{Q_{202}}{2}+\frac{Q_{022}}{2}-q_{012}^{2}\right) u_{3}^{2}+O(3) \\
\dot{u}_{3} & =\dot{u}_{2}\left(-q_{021} u_{2}-q_{012} u_{3}\right) \\
\dot{Q} & =D u_{2}-c Q+\left(-q_{102} q_{021}+Q_{121}\right) u_{3}+\left(-q_{201} q_{021}+C\right) u_{1}+Q^{2} a+ \\
& +\left(q_{201} q_{012}-Q_{211}\right) q_{021} u_{1} u_{2}+(2 B-D) u_{1} Q+\left(q_{201} q_{012}-Q_{211}\right) q_{012} u_{1} u_{3}+ \\
& +\left(\left(2 q_{012} q_{102}-Q_{112}\right) q_{021}+\left(q_{102} q_{021}-Q_{121}\right) q_{012}\right) u_{2} u_{3}+ \\
& +\left(\left(q_{021}-q_{201}\right) q_{021}-q_{201} q_{021}+2 C+2 q_{021}^{2}-E\right) Q u_{2}+ \\
& +\left(-q_{201} q_{012}+2 Q_{211}-Q_{031}+2 q_{021} q_{012}+\left(q_{021}-q_{201}\right) q_{012}\right) Q u_{3}+ \\
& +\left(2 q_{012} q_{102}-Q_{112}\right) q_{012} u_{3}^{2}+\left(q_{102} q_{021}-Q_{121}\right) q_{021} u_{2}^{2}+O(3)
\end{aligned}\right.
$$

Consideremos o campo vetorial de Lie-Cartan $X$ dado em (4.12). Os equilíbrios de $X$ são dados por

$$
\left\{\begin{array}{l}
L_{r}\left(u_{1}, u_{2}, u_{3}\right)=0 \\
M_{r}\left(u_{1}, u_{2}, u_{3}\right)=0 \\
A_{3}\left(u_{1}, u_{2}, u_{3}\right) P^{3}+A_{2}\left(u_{1}, u_{2}, u_{3}\right) P^{2}+A_{1}\left(u_{1}, u_{2}, u_{3}\right) P+A_{0}\left(u_{1}, u_{2}, u_{3}\right)=0
\end{array}\right.
$$

onde $A_{3}(0,0,0)=0, A_{2}(0,0,0)=-c, A_{1}(0,0,0)=a$ e $A_{0}(0,0,0)=0$. 
Como ja vimos, as equações $L_{r}\left(u_{1}, u_{2}, u_{3}\right)=0$ e $M_{r}\left(u_{1}, u_{2}, u_{3}\right)=0$ definem os pontos parcialmente umbílicos. Assim,

- Se $p$ é do tipo $D_{p}^{1}$ então o conjunto dos equilíbrios de $X$ é constituído por três pontos localizados em $P=0, P=\frac{a}{c}$ e $P=\infty$.

- Se $p$ é do tipo $D_{c}^{1}$ então o conjunto dos equilíbrios de $X$ é constituído por:

- Duas curvas regulares se intersectando transversalmente em $u_{1}=u_{2}=u_{3}=$ $0=P$,

- Duas curvas regulares se intersectando transversalmente em $u_{1}=u_{2}=u_{3}=$ $0, P=\frac{a}{c} \mathrm{e}$

- Duas curvas regulares se intersectando transversalmente em $u_{1}=u_{2}=u_{3}=$ $0, P=\infty$.

Observação 27 Sem perda de generalidade iremos supor que $a, b>0$.

Em $P=\frac{a}{c}$ a hipersuperfície de Lie-Cartan é regular. De fato, fazendo $q_{111}=0=$ $b=k$ em (4.8), (4.2) e (4.3) obtemos que

$$
\frac{\partial \mathcal{L}}{\partial u_{2}}\left(0,0,0, \frac{a}{c}\right)=a
$$


Pontos Parcialmente Umbílicos $D_{p}^{1}$ e $D_{c}^{1}$

A solução $u_{2}=u_{2}\left(u_{1}, u_{3}, P\right)$ da equação implícita $\mathcal{L}\left(u_{1}, u_{2}\left(u_{1}, u_{3}, P\right), u_{3}, P\right)=0$ é dada por

$$
\begin{aligned}
& u_{2}\left(u_{1}, u_{3}, P\right)=\frac{1}{2} \frac{a}{c} u_{1}+\frac{1}{2} \frac{\left(-2 a c^{3} q_{021}+2 a c^{3} q_{201}\right)}{a c^{4}} u_{3}+ \\
& +\frac{1}{a c^{4}}\left(2 q_{201} q_{021} a c^{3}-2 a^{3} q_{201} q_{021} c+3 a^{3} C c-3 D a^{2} c^{2}-+3 a^{2} B c^{2}\right. \\
& \left.-B c^{4}-3 C a c^{3}+a c^{3} A-2 a c^{3} q_{201}^{2}+a^{4} D-a^{3} E c+2 a^{3} q_{021}^{2} c\right) u_{1}^{2}+ \\
& +\frac{1}{2 a c^{4}}\left(-2 c^{4} Q_{211}+2 q_{201} q_{012} c^{4}+2 Q_{121} a^{3} c-2 c^{3} q_{021}^{2} q_{201}+2 c^{3} C q_{021}-\right. \\
& -2 B c^{2} a q_{021}-4 D c^{2} a q_{201}-4 Q_{121} c^{3} a+4 D c^{2} a q_{021}-2 a^{2} q_{201} q_{012} c^{2}- \\
& -4 c^{3} q_{201} q_{102} a-4 a^{2} c q_{021}^{3}+2 Q_{301} c^{3} a+2 B c^{2} a q_{201}+4 C a^{2} c\left(q_{201}-q_{021}\right)+ \\
& +6 a^{2} q_{201} q_{021}^{2} c+2 a^{2} c E q_{021}-2 q_{102} q_{021} a^{3} c-2 a^{2} q_{201}^{2} q_{021} c+2 a c^{3} q_{102} q_{021}- \\
& -2 a^{2} c E q_{201}+4 a^{2} c^{2} q_{021} q_{012}+4 Q_{211} a^{2} c^{2}-2 a^{2} c^{2} Q_{031}+2 D a^{3} q_{201}+ \\
& \left.+2 q_{201}^{2} q_{021} c^{3}-2 c^{3} C q_{201}-2 D a^{3} q_{021}\right) u_{1} u_{3}+\frac{1}{2 a c^{4}}\left(D a^{2} q_{201}^{2}-a c^{3} Q_{022}+\right. \\
& +2 a c^{3} q_{012}^{2}+Q_{112} a^{2} c^{2}+2 a c q_{021}^{4}+2 q_{102} q_{021} c^{3} q_{201}-2 Q_{121} a^{2} c q_{021}+ \\
& +2 D c^{2} q_{021} q_{201}-2 q_{102} q_{012} a^{2} c^{2}+4 a c^{2} q_{021} q_{012} q_{201}-4 a c^{2} q_{021}^{2} q_{012}- \\
& -a c E q_{021}^{2}+2 a c q_{021}^{2} q_{201}^{2}-a c E q_{201}^{2}+C a c q_{021}^{2}+2 q_{102} q_{021}^{2} a^{2} c+ \\
& +C a c q_{201}^{2}+2 a c^{2} Q_{031} q_{021}+2 Q_{211} a c^{2} q_{201}-Q_{112} c^{4}+2 Q_{121} a^{2} c q_{201}- \\
& +2 a c E q_{021} q_{201}-2 C a c q_{021} q_{201}-2 q_{102} q_{021} a^{2} c q_{201}-D c^{2} q_{021}^{2}-D c^{2} q_{201}^{2}- \\
& -2 Q_{121} c^{3} q_{201}+2 q_{012} q_{102} c^{4}-2 q_{102} q_{021}^{2} c^{3}+a c^{3} Q_{202}+2 Q_{121} c^{3} q_{021}- \\
& -2 a c^{2} Q_{031} q_{201}-2 D a^{2} q_{021} q_{201}+D a^{2} q_{021}^{2}-2 a c^{3} q_{102}^{2}-2 Q_{211} a c^{2} q_{021}- \\
& \left.-4 a c q_{021}^{3} q_{201}\right) u_{3}^{2}+O(3)
\end{aligned}
$$


Próximo ao ponto $\left(0,0,0, \frac{a}{c}\right)$, o campo vetorial $X$ restrito a hipersuperfície de Lie-Cartan é dado por

$$
X_{\mathcal{L}}=\left\{\begin{aligned}
\dot{u}_{1} & =c u_{2}\left(u_{1}, u_{3}, P\right)+\left(-q_{201}+q_{021}\right) u_{3}-a u_{1}+ \\
& +\left(q_{201}^{2}-\frac{A}{2}+\frac{C}{2}\right) u_{1}^{2}+(-B+D) u_{2}\left(u_{1}, u_{3}, P\right) u_{1}+ \\
& +\left(2 q_{201} q_{102}-Q_{301}+Q_{121}\right) u_{1} u_{3}+ \\
& +\left(-q_{021}^{2}+\frac{E}{2}-\frac{C}{2}\right) u_{2}\left(u_{1}, u_{3}, P\right)^{2}+ \\
& +\left(-Q_{211}+Q_{031}-2 q_{021} q_{012}\right) u_{2}\left(u_{1}, u_{3}, P\right) u_{3}+ \\
& +\left(q_{102}^{2}-\frac{Q_{202}}{2}+\frac{Q_{022}}{2}-q_{012}^{2}\right) u_{3}^{2}+O(3) \\
\dot{u}_{3} & =\dot{u}_{1}\left(-q_{201} u_{1}-q_{102} u_{3}+O(2)\right) \\
\dot{P} & =\left(q_{201} q_{021}-C\right) u_{2}\left(u_{1}, u_{3}, P\right)+\left(q_{201} q_{012}-Q_{211}\right) u_{3}-B u_{1}+a P+ \\
& +\left(-3 q_{201} q_{102}+Q_{301}-2 Q_{121}+2 q_{102} q_{021}\right) P u_{3}+ \\
& +(B-2 D) P u_{2}\left(u_{1}, u_{3}, P\right)+\left(Q_{121} q_{201}-q_{102} q_{021} q_{201}\right) u_{1} u_{2}\left(u_{1}, u_{3}, P\right)+ \\
& +\left(-q_{102}^{2} q_{021}+q_{102} Q_{121}\right) u_{2}\left(u_{1}, u_{3}, P\right) u_{3}-c P^{2}+ \\
& +\left(-q_{201}^{2} q_{012}+Q_{211} q_{201}\right) u_{1}^{2}+\left(q_{201} Q_{112}-3 q_{201} q_{012} q_{102}+q_{102} Q_{211}\right) u_{1} u_{3}+ \\
& +\left(-3 q_{201}^{2}+2 q_{201} q_{021}+A-2 C\right) P u_{1}+\left(-2 q_{102}^{2} q_{012}+q_{102} Q_{112}\right) u_{3}^{2}+O(3)
\end{aligned}\right.
$$

Disto os autovalores de $D X_{\mathcal{L}}\left(0,0, \frac{a}{c}\right)$ são $0,0,-a$.

Observação 28 O eixo $P$ é o autoespaço associado ao autovalor $-a$.

Estudaremos $X_{\mathcal{L}}$ restrito a variedade central, que neste caso tem dimensão 2 .

Escrevendo a variedade central como gráfico sobre as variáveis $u_{1}, u_{3}: P=$ $P\left(u_{1}, u_{3}\right)$, obtemos que o campo $X_{\mathcal{L}}$ restrito a variedade central satisfaz

$$
\left.X_{\mathcal{L}}\right|_{W^{c}}=\left\{\begin{array}{l}
\dot{u}_{1}=B\left(u_{1}, u_{3}\right) \\
\dot{u}_{3}=B\left(u_{1}, u_{3}\right) C\left(u_{1}, u_{3}\right)
\end{array}\right.
$$

onde

$$
\operatorname{det}\left(\left.\left(\operatorname{Hessiana}\left(B\left(u_{1}, u_{3}\right)\right)\right)\right|_{u_{1}=0=u_{3}}\right)=\frac{\zeta}{4 a^{2} c^{4}} \text {. }
$$

Assim, o retrato de fase de $\left.X_{\mathcal{L}}\right|_{W^{c}}$ é como na figura 4.3 se $p$ é do tipo $D_{c}^{1}(\zeta<0)$, e como na figura 4.4 se $p$ é do tipo $D_{p}^{1}$. 


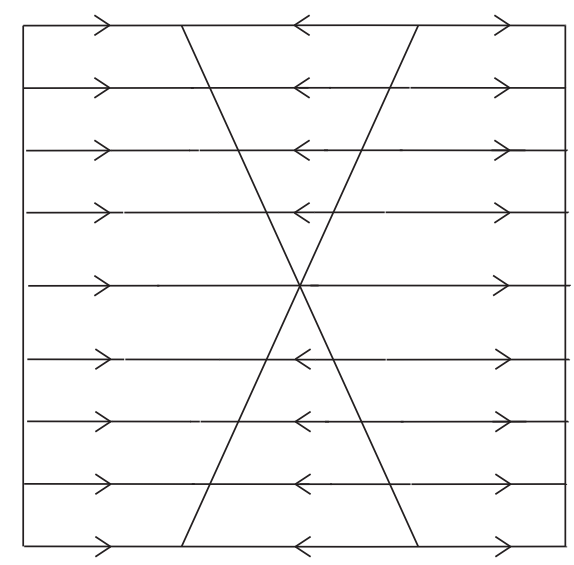

Figura 4.3: Comportamento de $\left.X_{\mathcal{L}}\right|_{W^{c}}$ quando $\zeta<0$

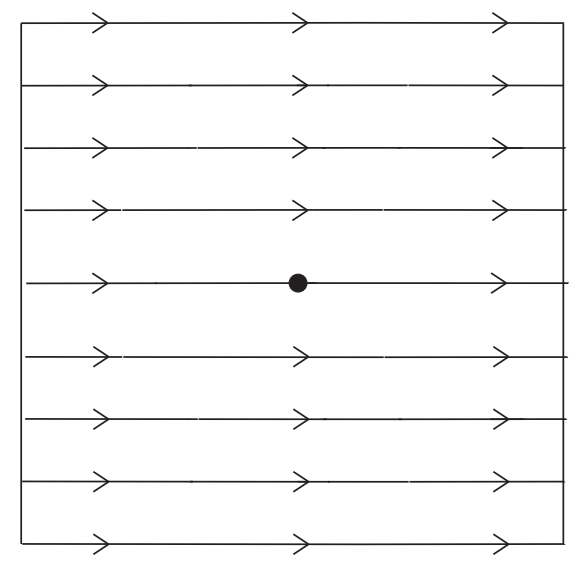

Figura 4.4: Comportamento de $\left.X_{\mathcal{L}}\right|_{W^{c}}$ quando $\zeta>0$

Quanto aos pontos $P=0$ e $P=\infty(Q=0)$ teremos que

- os autovalores de $D X(0,0,0,0)$ são $0,0, a,-a$

- os autovalores de $D Y(0,0,0,0)$ são $0,0, c,-c$

Já vimos que a hipersuperfície de Lie-Cartan tem uma singularidade do tipo Morse em $P=0$ e $P=\infty(Q=0)$.

Observação 29 A variedade central de $X$, respectivamente de $Y$, numa vizinhança de $P=0$, respectivamente $Q=0$, intersecta a hipersuperfície de Lie-Cartan nos respectivos equilíbrios. 
Assim da análise feita acima, concluímos a demonstração dos teoremas 10 e 11 . 


\section{Capítulo 5}

\section{Teorema de Genericidade}

Uma família a um parâmetro de hipersuperfícies imersas será uma família $\alpha_{\lambda}$ de imersões de uma hipersuperfície orientada $\mathbb{M}^{3}$ no espaço $\mathbb{R}^{4}$, onde $\lambda \in \mathbb{R}$. A família $\alpha_{\lambda}$ será dita suave se $\alpha(m, \lambda)=\alpha_{\lambda}(m)$ é de classe $C^{\infty}$ no produto $\mathbb{M}^{3} \times \mathbb{R}$. O espaço destas famílias, denotado por $\mathcal{F}_{\mathbb{M}^{3} \times \mathbb{R}}$ será dotado da topologia de Whitney. O termo genérico, usado para nos referirmos a uma propriedade da família, significa que é válida para uma coleção de famílias que contém a interseção enumerável de abertos densos em $\mathcal{F}_{\mathbb{M}^{3} \times \mathbb{R}}$. Pelo teorema de Baire esta coleção será densa. Ver [17].

Consideremos o espaço $\mathbb{J}^{r}\left(\mathbb{M}^{3}, \mathbb{R}^{4}\right)$ dos $r$-jatos das imersões, $\alpha$, de $\mathbb{M}^{3}$ em $\mathbb{R}^{4}$, dotado com a estrutura de fibrado principal. O espaço base é $\mathbb{M}^{3} \times \mathbb{R}^{4}$, a fibra é o espaço $\mathcal{J}^{r}(3,4)$, onde $\mathcal{J}^{r}(3,4)$ é o espaço dos $r$-jatos de imersões de $\mathbb{R}^{3}$ em $\mathbb{R}^{4}$, preservando as respectivas origens. O grupo estrutural, $\mathcal{A}_{+}^{r}$, é o produto do grupo $\mathcal{L}_{+}^{r}(3,3)$ dos $r$-jatos de difeomorfismos preservando a origem e a orientação do $\mathbb{R}^{3}$, agindo à direita por mudança de coordenadas, e o grupo $\mathcal{O}_{+}(4,4)$ das isometrias positivas, a ação à esquerda consiste de uma rotação positiva no $\mathbb{R}^{4}$.

Cada 5-jato de uma imersão em um ponto parcialmente umbílico é da forma $(p, \tilde{p}, w) \operatorname{com}(p, \tilde{p}) \in \mathbb{M}^{3} \times \mathbb{R}^{4} \mathrm{e} w$ na órbita de uma imersão polinomial $\left(u_{1}, u_{2}, u_{3}, h\left(u_{1}, u_{2}, u_{3}\right)\right)$, 
onde

$$
\begin{aligned}
h\left(u_{1}, u_{2}, u_{3}\right)= & \frac{k}{2}\left(u_{1}^{2}+u_{2}^{2}\right)+\frac{k_{3}}{2} u_{3}^{2}+\frac{a}{6} u_{1}^{3}+\frac{b}{2} u_{1} u_{2}^{2}+\frac{c}{6} u_{2}^{3}+\frac{q_{003}}{6} u_{3}^{3}+\frac{q_{012}}{2} u_{2} u_{3}^{2} \\
& +q_{111} u_{1} u_{2} u_{3}+\frac{q_{021}}{2} u_{2}^{2} u_{3}+\frac{q_{102}}{2} u_{1} u_{3}^{2}+\frac{q_{201}}{2} u_{1}^{2} u_{3}+\frac{A}{24} u_{1}^{4}+\frac{B}{6} u_{1}^{3} u_{2} \\
& +\frac{C}{4} u_{1}^{2} u_{2}^{2}+\frac{D}{6} u_{1} u_{2}^{3}+\frac{E}{24} u_{2}^{4}+\frac{Q_{004}}{24} u_{3}^{4}+\frac{Q_{013}}{6} u_{3}^{3} u_{2}+\frac{Q_{103}}{6} u_{3}^{3} u_{1}+ \\
& +\frac{Q_{022}}{4} u_{2}^{2} u_{3}^{2}+\frac{Q_{202}}{4} u_{1}^{2} u_{3}^{2}+\frac{Q_{112}}{2} u_{3}^{2} u_{1} u_{2}+\frac{Q_{031}}{6} u_{3} u_{2}^{3}+\frac{Q_{301}}{6} u_{1}^{3} u_{3}+ \\
& +\frac{Q_{121}}{2} u_{3} u_{2}^{2} u_{1}+\frac{Q_{211}}{2} u_{3} u_{2} u_{1}^{2}+\frac{a_{500}}{120} u_{1}^{5}+\frac{a_{410}}{24} u_{1}^{4} u_{2}+\frac{a_{320}}{12} u_{1}^{3} u_{2}^{2}+ \\
& +\frac{a_{230}}{12} u_{1}^{2} u_{2}^{3}+\frac{a_{140}}{24} u_{1} u_{2}^{4}+\frac{a_{050}}{120} u_{2}^{5}+\frac{a_{401}}{24} u_{1}^{4} u_{3}+\frac{a_{302}}{12} u_{1}^{3} u_{3}^{2}+ \\
& +\frac{a_{203}}{12} u_{1}^{2} u_{3}^{3}+\frac{1}{24} a_{104} u_{1} u_{3}^{4}+\frac{1}{120} a_{005} u_{3}^{5}+\frac{1}{24} a_{041} u_{2}^{4} u_{3}+\frac{a_{032}}{12} u_{2}^{3} u_{3}^{2} \\
& +\frac{a_{023}}{12} u_{2}^{2} u_{3}^{3}+\frac{a_{014}}{24} u_{2} u_{3}^{4}+\frac{a_{311}}{6} u_{1}^{3} u_{2} u_{3}+\frac{a_{131}}{6} u_{1} u_{2}^{3} u_{3}+\frac{a_{113}}{6} u_{1} u_{2} u_{3}^{3}+ \\
& +\frac{a_{221}}{4} u_{1}^{2} u_{2}^{2} u_{3}+\frac{a_{212}}{4} u_{1}^{2} u_{2} u_{3}^{2}+\frac{1}{4} a_{122} u_{1} u_{2}^{2} u_{3}^{2}+O(6)
\end{aligned}
$$

A parte quadrática geral de $\tilde{h}$ onde $\left(u_{1}, u_{2}, u_{3}, \tilde{h}\right)$ está na órbita da $h$ tem a forma

$$
k_{110} u_{1} u_{2}+k_{101} u_{1} u_{3}+k_{011} u_{2} u_{3}+\frac{k_{200}}{2} u_{1}^{2}+\frac{k_{020}}{2} u_{2}^{2}+\frac{k_{002}}{2} u_{3}^{2} .
$$

A variedade dos jatos parcialmente umbílicos, $(\mathcal{P U})^{5}$, é definida pela condição de que a matriz simétrica

$$
\left(\begin{array}{lll}
k_{200} & k_{110} & k_{101} \\
k_{110} & k_{020} & k_{011} \\
k_{101} & k_{011} & k_{002}
\end{array}\right)
$$

possui dois autovalores iguais.

Em [6], R. Garcia mostrou que $(\mathcal{P U})^{5}$ é uma subvariedade de codimensão $2 \mathrm{em}$ $\mathcal{J}^{5}(3,4)$.

A variedade dos jatos umbílicos, $\mathcal{U}^{5}$, é definida pela condição de que na matriz acima $k_{200}=k_{020}=k_{002}$ e $k_{110}=k_{101}=k_{011}=0$. Esta forma uma subvariedade 
fechada de codimensão 5 em $\mathcal{J}^{5}(3,4)$.

Observação 30 A expressão na equação (5.1) é um representante da órbita dos jatos parcialmente umbílicos onde o termo $\frac{d}{2} u_{1}^{2} u_{2}$ pode ser considerado nulo por uma rotação.

Definiremos a seguir a estratificação parcialmente umbílica canônica. O termo canônica significa que os estratos são invariantes pela ação do grupo $\mathcal{A}_{+}^{5}=\mathcal{O}_{+}(4,4) \times$ $\mathcal{L}_{+}^{5}(3,3)$. É com relação a órbita desta ação que faremos referência a seguir.

1. Jatos Umbílicos: $\mathcal{U}^{5}$, é uma subvariedade fechada de codimensão 5 em $\mathcal{J}^{5}(3,4)$.

2. Jatos Parcialmente Umbilicos: $(\mathcal{P U})^{5}$, aqueles na órbita de $\mathcal{J}^{5}\left(u_{1}, u_{2}, u_{3}, h\right)$, onde $h=h\left(u_{1}, u_{2}, u_{3}\right)$ é como na equação (5.1), com $k \in \mathbb{R}$. É uma subvariedade de codimensão 2 em $\mathcal{J}^{r}(3,4)$.

3. $\mathcal{Q}^{5}$ : Aqueles na órbita de $\mathcal{J}^{5}\left(u_{1}, u_{2}, u_{3}, h\right)$, com $h=h\left(u_{1}, u_{2}, u_{3}\right)$ como na equação (5.1), onde $a=0=b=c$. É uma subvariedade fechada de codimensão $3 \mathrm{em}$ $(\mathcal{P U})^{5}$ e de codimensão 5 em $\mathcal{J}^{5}(3,4)$.

4. Jatos Parcialmente Umbilicos Não Darbouxianos: $\mathcal{N D}^{5}$, aqueles na órbita de $\mathcal{J}^{5}\left(u_{1}, u_{2}, u_{3}, h\right)$, com $h=h\left(u_{1}, u_{2}, u_{3}\right)$ como na equação (5.1) satisfazendo

$$
\begin{gathered}
b(b-a)=0 \text { ou } \\
b(b-a) \neq 0 \text { e } a-2 b=0 \text { ou } c^{2}-a b(a-2 b)=0 .
\end{gathered}
$$

Esta variedade pode ser particionada como segue:

(a) $\left(\mathcal{D}_{12}\right)^{5}$ : Definida pela órbita dos jatos com

$$
\left\{\begin{array}{c}
a-2 b=0 \text { e } \chi_{2} \neq 0, \text { ou } \\
c^{2}-4 b(a-2 b)=0 \text { e } \chi_{1} \neq 0
\end{array}\right.
$$

(b) $\left(\mathcal{D}_{23}\right)^{5}$ : Definida pela órbita dos jatos com $a=b \neq 0$ e $\chi \neq 0$

(c) $\left(\mathcal{D}_{1}^{1}\right)^{5}$ : Definida pela órbita dos jatos $\operatorname{com} c=0, a=2 b \neq 0$ e $\tilde{\chi}_{1} \neq 0$

(d) $\left(\mathcal{D}_{2}^{1}\right)^{5}$ : Definida pela órbita dos jatos com $a=b \neq 0, \chi=0$ e $\tilde{\chi}<0$

(e) $\left(\mathcal{D}_{3}^{1}\right)^{5}$ : Definida pela órbita dos jatos com $a=b \neq 0, \chi=0$ e $\tilde{\chi}>0$

(f) $\left(\mathcal{D}_{13}^{1}\right)^{5}$ : Definida pela órbita dos jatos com $a=b=0$ e $\frac{B k-B+3 q_{111} q_{201}}{k-1} \neq$ 0 
(g) $\left(\mathcal{D}_{p}^{1}\right)^{5}$ : Definida pela órbita dos jatos $\operatorname{com} b=q_{111}=0$ e $\zeta>0$

(h) $\left(\mathcal{D}_{c}^{1}\right)^{5}$ : Definida pela órbita dos jatos com $b=q_{111}=0$ e $\zeta<0$

(i) $\left(\mathcal{D}_{1 h, n}^{1}\right)^{5}$ : Definida pela órbita dos jatos com $a-2 b=0, \chi_{2}=0$ e $\tilde{\chi}_{2}<0$

(j) $\left(\mathcal{D}_{1 h, p}^{1}\right)^{5}$ : Definida pela órbita dos jatos com $a-2 b=0, \chi_{2}=0$ e $\tilde{\chi}_{2}>0$

(k) $\mathcal{Z}^{5}$ : Definida pela órbita dos jatos com $a=b \neq 0, \chi=0$ e $\tilde{\chi}=0$

(1) $\mathcal{W}^{5}$ : Definida pela órbita dos jatos com $a=b=0$ e $\frac{B k-B+3 q_{111} q_{201}}{k-1}=0$

5. Jatos Parcialmente Umbilicos Darbouxianos: $\mathcal{D}^{5}$, o complementar em $(\mathcal{P U})^{5}$ de $\mathcal{N D}^{5}$, aqueles na órbita de $\mathcal{J}^{5}\left(u_{1}, u_{2}, u_{3}, h\right)$, com $h=h\left(u_{1}, u_{2}, u_{3}\right)$ como na equação (5.1), onde

(a) $\left(\mathcal{D}_{1}\right)^{5}:\left(\frac{c}{2 b}\right)^{2}-\frac{a}{b}+2<0$

(b) $\left(\mathcal{D}_{2}\right)^{5}:\left(\frac{c}{2 b}\right)^{2}+2>\frac{a}{b}>1, a \neq 2 b$

(c) $\left(\mathcal{D}_{3}\right)^{5}: \frac{a}{b}<1$.

A estratificação canônica de $\mathcal{J}^{5}(3,4)$ induz uma estratificação canônica de $\rrbracket^{5}\left(\mathbb{M}^{3}, \mathbb{R}^{4}\right)$ cujos estratos são subfibrados principais, com codimensão igual a das suas fibras, que são os estratos canônicos de $\mathcal{J}^{5}(3,4)$ como definidos acima nos itens $1,2,3,4$ e 5 .

A coleção dos subfibrados que estratificam $\mathbb{J}^{5}\left(\mathbb{M}^{3}, \mathbb{R}^{4}\right)$ será chamada estratificação parcialmente umbílica. Os estratos são: $\mathbb{P U}^{5}\left(\mathbb{M}^{3}, \mathbb{R}^{4}\right)$ correspondente a $(\mathcal{P U})^{5}$; $\left(\mathbb{D}_{i}\right)^{5}\left(\mathbb{M}^{3}, \mathbb{R}^{4}\right), i=1,2,3$, correspondente ao estrato dos jatos parcialmente umbílicos Darbouxianos $\left(\mathcal{D}_{i}\right)^{5}, i=1,2,3$, e assim sucessivamente, um fibrado para cada estrato listado acima.

Teorema 12 As seguintes propriedades são genéricas para famílias a um parâmetro, $\alpha_{\lambda}$, de imersões em $\mathcal{F}_{\mathbb{M}^{3} \times \mathbb{R}}$. O conjunto $\mathbb{P U}\left(\alpha_{\lambda}\right)$ de pontos $(p, \lambda)$ em $\mathbb{M}^{3} \times \mathbb{R}$ tal que $p$ é um ponto parcialmente umbilico de $\alpha_{\lambda}$, forma uma subvariedade, de $\mathbb{M}^{3} \times \mathbb{R}$, diferenciável e de dimensão 2, estratificada como segue

- Os pontos parcialmente umbílicos Darbouxianos, $D_{1}, D_{2}$ e $D_{3}$, ocorrem ao longo de subvariedades de $\mathbb{P U}\left(\alpha_{\lambda}\right)$ de codimensão 0

- Os pontos parcialmente umbílicos não Darbouxianos, $D_{12}$ e $D_{23}$, ocorrem ao longo de subvariedades de $\mathbb{P U}\left(\alpha_{\lambda}\right)$ de codimensão 1 
- Os pontos parcialmente umbílicos não-Darbouxianos, $D_{1}^{1}, D_{2}^{1}, D_{3}^{1}, D_{13}^{1}, D_{1 h}^{1}, D_{p}^{1}$ e $D_{c}^{1}$, ocorrem ao longo de subvariedades de $\mathbb{P U}\left(\alpha_{\lambda}\right)$ de codimensão 2.

Demonstração: Basta aplicar o teorema da transversalidade de Thom ao espaço dos jatos. Neste caso, $j^{5} \alpha_{\lambda}$ é um mapa definido em $\mathbb{M}^{3} \times \mathbb{R}$ transversal à estratificação canônica de $\mathbb{J}^{5}\left(\mathbb{M}^{3}, \mathbb{R}^{4}\right)$. Observamos que os estratos não listados no enunciado do teorema tem codimensão maior do que 4 e, portanto, são evitados pela transversalidade.

Assim, com o teorema 12 concluímos que os pontos parcialmente umbílicos que aparecem genericamente em famílias a um parâmetro de hipersuperfícies imersas em $\mathbb{R}^{4}$, são os pontos $D_{1}^{1}, D_{2}^{1}, D_{3}^{1}, D_{13}^{1}, D_{1 h, n}^{1}, D_{1 h, p}^{1}, D_{p}^{1}$ e $D_{c}^{1}$. 


\section{Capítulo 6}

\section{Diagramas de Bifurcação dos Pontos Parcialmente Umbílicos $D_{1}^{1}, D_{2}^{1}, D_{3}^{1}$, $D_{13}^{1}, D_{1 h, n}^{1}, D_{1 h, p}^{1}, D_{p}^{1}$ e $D_{c}^{1}$}

Neste capítulo, estudaremos as mudanças qualitativas - bifurcações - que ocorrem na configuração principal dos pontos parcialmente umbílicos $D_{1}^{1}, D_{2}^{1}, D_{3}^{1}, D_{13}^{1}, D_{1 h, n}^{1}, D_{1 h, p}^{1}$, $D_{p}^{1}$ e $D_{c}^{1}$ definidos nos capítulos 3 e 4 .

\subsection{Preliminares}

Sejam $\alpha: \mathbb{M}^{3} \rightarrow \mathbb{R}^{4}$ uma imersão, em $\mathbb{R}^{4}$, de uma variedade de dimensão 3 , compacta, orientada e de classe $C^{k}(k \geq 5)$, e $p$ um ponto parcialmente umbílico de $\alpha$ de um dos seguintes tipos: $D_{1}^{1}, D_{2}^{1}, D_{3}^{1}, D_{13}^{1}, D_{1 h, n}^{1}, D_{1 h, p}^{1}, D_{p}^{1}$ ou $D_{c}^{1}$. Escrevemos

$$
\alpha\left(u_{1}, u_{2}, u_{3}\right)=\left(u_{1}, u_{2}, u_{3}, h\left(u_{1}, u_{2}, u_{3}\right)\right)
$$

onde 
Diagramas de bifurcação dos pontos $D_{1}^{1}, D_{2}^{1}, D_{3}^{1}, D_{13}^{1}, D_{1 h, n}^{1}, D_{1 h, p}^{1}, D_{p}^{1}$ e $D_{c}^{1}$

$$
\begin{aligned}
& h\left(u_{1}, u_{2}, u_{3}\right)=\frac{k}{2}\left(u_{1}^{2}+u_{2}^{2}\right)+\frac{1}{2} u_{3}^{2}+\frac{a}{6} u_{1}^{3}+\frac{b}{2} u_{1} u_{2}^{2}+\frac{c}{6} u_{2}^{3}+\frac{q_{003}}{6} u_{3}^{3} \\
& +\frac{1}{2} q_{012} u_{2} u_{3}^{2}+q_{111} u_{1} u_{2} u_{3}+\frac{1}{2} q_{021} u_{2}^{2} u_{3}+\frac{1}{2} q_{102} u_{1} u_{3}^{2} \\
& +\frac{1}{2} q_{201} u_{1}^{2} u_{3}+\frac{1}{24} A u_{1}^{4}+\frac{1}{6} B u_{1}^{3} u_{2}+\frac{1}{4} C u_{1}^{2} u_{2}^{2}+\frac{1}{6} D u_{1} u_{2}^{3} \\
& +\frac{1}{24} E u_{2}^{4}+\frac{1}{24} Q_{004} u_{3}^{4}+\frac{1}{6} Q_{013} u_{3}^{3} u_{2}+\frac{1}{6} Q_{103} u_{3}^{3} u_{1} \\
& +\frac{1}{4} Q_{022} u_{2}^{2} u_{3}^{2}+\frac{1}{4} Q_{202} u_{1}^{2} u_{3}^{2}+\frac{1}{2} Q_{112} u_{3}^{2} u_{1} u_{2}+\frac{1}{6} Q_{031} u_{3} u_{2}^{3} \\
& +\frac{1}{6} Q_{301} u_{1}^{3} u_{3}+\frac{1}{2} Q_{121} u_{3} u_{2}^{2} u_{1}+\frac{1}{2} Q_{211} u_{3} u_{2} u_{1}^{2}+ \\
& +\frac{1}{120} a_{500} u_{1}^{5}+\frac{1}{24} a_{410} u^{4} u_{2}+\frac{1}{12} a_{320} u_{1}^{3} u_{2}^{2}+\frac{1}{12} a_{230} u_{1}^{2} u_{2}^{3} \\
& +\frac{1}{24} a_{140} u_{1} u_{2}^{4}+\frac{1}{120} a_{050} u_{2}^{5}+\frac{1}{24} a_{401} u^{4} w+\frac{1}{12} a_{302} u_{1}^{3} u_{3}^{2} \\
& +\frac{1}{12} a_{203} u_{1}^{2} u_{3}^{3}+\frac{1}{24} a_{104} u_{1} u_{3}^{4}+\frac{1}{120} a_{005} u_{3}^{5}+\frac{1}{24} a_{041} u_{2}^{4} u_{3} \\
& +\frac{1}{12} a_{032} u_{2}^{3} u_{3}^{2}+\frac{1}{12} a_{023} u_{2}^{2} u_{3}^{3}+\frac{1}{24} a_{014} u_{2} u_{3}^{4}+\frac{1}{6} a_{311} u_{1}^{3} u_{2} u_{3} \\
& +\frac{1}{6} a_{131} u_{1} u_{2}^{3} u_{3}+\frac{1}{6} a_{113} u_{1} u_{2} u_{3}^{3}+\frac{1}{4} a_{221} u_{1}^{2} u_{2}^{2} u_{3}+\frac{1}{4} a_{212} u_{1}^{2} u_{2} u_{3}^{2} \\
& +\frac{1}{4} a_{122} u_{1} u_{2}^{2} u_{3}^{2}+O(6)
\end{aligned}
$$

e uma das seguintes condições é válida:

$\left.D_{1}^{1}\right)$ Se $c=0, a=2 b \neq 0$ então $\tilde{\chi}_{1} \neq 0$, onde

$$
\begin{aligned}
\tilde{\chi}_{1}= & q_{111} k^{4}-q_{111} k^{3}+Q_{211} b k+q_{021} B k-q_{201} B k-q_{111} C k-q_{021} B \\
& -2 q_{111}{ }^{3}+b q_{012} q_{201}-3 q_{201}{ }^{2} q_{111}+q_{111} C-Q_{211} b+q_{201} B \\
& +2 q_{111} b q_{102}+2 q_{201} q_{111} q_{021}
\end{aligned}
$$


Diagramas de bifurcação dos pontos $D_{1}^{1}, D_{2}^{1}, D_{3}^{1}, D_{13}^{1}, D_{1 h, n}^{1}, D_{1 h, p}^{1}, D_{p}^{1}$ e $D_{c}^{1}$

$\left.D_{2}^{1}\right)$ Se $b=a \neq 0, \chi=0$ então $\tilde{\chi}<0$,

$\left.D_{3}^{1}\right)$ Se $b=a \neq 0, \chi=0$ então $\tilde{\chi}>0$, onde

$$
\chi=C-A-\frac{c B}{b}-2 \frac{c q_{111} q_{201}}{(k-1) b}+2 k^{3}-\frac{q_{201}^{2}-2 q_{111}^{2}}{k-1}
$$

e

$$
\begin{aligned}
\tilde{\chi}= & \frac{a_{410} c}{b}-a_{500}+a_{320}-\frac{3 D B}{b}+\frac{3 c C B}{b^{2}}+\frac{3 B^{2}}{b}+12 k^{2} b-\left(\frac{3 c B}{b^{2}}-\frac{6 q_{201} c q_{111}}{(k-1) b^{2}}\right) k^{3}+ \\
& +\frac{6 q_{201}^{2} b}{(k-1)^{2}}+\frac{6 q_{201}^{2} c q_{111} q_{021}}{(k-1)^{2} b^{2}}+\frac{6 Q_{211} q_{111}}{k-1}+\frac{3 c B q_{111}^{2}}{(k-1) b^{2}}+\frac{6 q_{201} q_{111} c}{(k-1)^{2}}+ \\
& +\frac{12 q_{111}^{2} q_{201}^{2}}{(k-1)^{2} b}-\frac{6 q_{201} q_{111} D}{(k-1) b}-\frac{6 q_{201}^{2} q_{102}}{(k-1)^{2}}+\frac{3 q_{201} c B q_{021}}{(k-1) b^{2}}+\frac{12 q_{201} B q_{111}}{(k-1) b}+ \\
& +\frac{6 q_{201} c q_{111}^{3}}{(k-1)^{2} b^{2}}-\frac{12 q_{021} q_{111}^{2} q_{201}}{(k-1)^{2} b}-\frac{6 q_{201} q_{102} c q_{111}}{(k-1)^{2} b}+\frac{6 q_{102} q_{111}^{2}}{(k-1)^{2}}-\frac{3 q_{201} c Q_{211}}{(k-1) b}+ \\
& +\frac{6 q_{201} q_{111} c C}{(k-1) b^{2}}-\frac{3 c q_{111} Q_{301}}{(k-1) b}-\frac{6 q_{111}^{2} b}{(k-1)^{2}}-\frac{6 q_{021} q_{111} B}{(k-1) b}
\end{aligned}
$$

$\left.D_{13}^{1}\right)$ Se $a=b=0$ então $c q_{111} \neq 0$ e $B k-B+3 q_{111} q_{201} k-1 \neq 0$, ou se $c=b=0$ então $a q_{111} \neq 0$ e $\frac{B k-B+3 q_{111} q_{201}}{k-1} \neq 0$,

$\left.D_{1 h, p}^{1}\right) c b(b-a) \neq 0, \frac{a}{b}=2, \chi_{2}=0$ e $\tilde{\chi}_{2}>0$

$\left.D_{1 h, n}^{1}\right) c b(b-a) \neq 0, \frac{a}{b}=2, \chi_{2}=0$ e $\tilde{\chi}_{2}<0$ (Veja equações (3.51) e (3.52) no capítulo $3)$,

$\left.D_{p}^{1}\right) b=q_{111}=0$ e $\zeta>0$

$\left.D_{c}^{1}\right) b=q_{111}=0$ e $\zeta<0$ (Veja equação $(4.7$ no capítulo 4$)$ ).

Consideremos uma família a 1 parâmetro de imersões $\alpha_{\lambda}$ tal que $\alpha_{0}=\alpha$. Esta pode ser escrita da forma

$$
\begin{aligned}
\alpha_{\lambda}\left(u_{1}, u_{2}, u_{3}\right)= & \left(u_{1}+\mathcal{U}_{1}\left(u_{1}, u_{2}, u_{3}, \lambda\right), u_{2}+\mathcal{U}_{2}\left(u_{1}, u_{2}, u_{3}, \lambda\right)\right. \\
& \left.u_{3}+\mathcal{U}_{3}\left(u_{1}, u_{2}, u_{3}, \lambda\right), h\left(u_{1}, u_{2}, u_{3}\right)+\mathcal{H}\left(u_{1}, u_{2}, u_{3}, \lambda\right)\right)
\end{aligned}
$$


Diagramas de bifurcação dos pontos $D_{1}^{1}, D_{2}^{1}, D_{3}^{1}, D_{13}^{1}, D_{1 h, n}^{1}, D_{1 h, p}^{1}, D_{p}^{1}$ e $D_{c}^{1}$

onde $\mathcal{U}_{1}\left(u_{1}, u_{2}, u_{3}, 0\right)=0, \mathcal{U}_{2}\left(u_{1}, u_{2}, u_{3}, 0\right)=0, \mathcal{U}_{3}\left(u_{1}, u_{2}, u_{3}, 0\right)=0$ e $\mathcal{H}\left(u_{1}, u_{2}, u_{3}, 0\right)=$ 0 .

Fazendo a mudança de coordenadas

$$
\left\{\begin{array}{l}
\tilde{u}_{1}=u_{1}+\mathcal{U}_{1}\left(u_{1}, u_{2}, u_{3}, \lambda\right) \\
\tilde{u}_{2}=u_{2}+\mathcal{U}_{2}\left(u_{1}, u_{2}, u_{3}, \lambda\right) \\
\tilde{u}_{3}=u_{3}+\mathcal{U}_{3}\left(u_{1}, u_{2}, u_{3}, \lambda\right)
\end{array}\right.
$$

obtemos que, nestas novas coordenadas, (6.4) é dada por

$$
\alpha_{\lambda}\left(\tilde{u}_{1}, \tilde{u}_{2}, \tilde{u}_{3}\right)=\left(\tilde{u}_{1}, \tilde{u}_{2}, \tilde{u}_{3}, \tilde{H}\left(\tilde{u}_{1}, \tilde{u}_{2}, \tilde{u}_{3}, \lambda\right)\right) \text {. }
$$

Eliminando os termos lineares da $\tilde{H}$, com uma rotação, podemos supor que

$$
\begin{aligned}
\tilde{H}\left(\tilde{u}_{1}, \tilde{u}_{2}, \tilde{u}_{3}, \lambda\right) & =\frac{a_{200}(\lambda)}{2} \tilde{u}_{1}^{2}+\frac{a_{020}(\lambda)}{2} \tilde{u}_{2}^{2}+\frac{a_{002}(\lambda)}{2} \tilde{u}_{3}^{2}+a_{110}(\lambda) \tilde{u}_{1} \tilde{u}_{2}+ \\
& +a_{101}(\lambda) \tilde{u}_{1} \tilde{u}_{3}+a_{011}(\lambda) \tilde{u}_{2} \tilde{u}_{3}+\frac{a(\lambda)}{6} \tilde{u}_{1}^{3}+\frac{b(\lambda)}{2} \tilde{u}_{1} \tilde{u}_{2}^{2}+ \\
& +\frac{d(\lambda)}{2} \tilde{u}_{1}^{2} \tilde{u}_{2}+\frac{c(\lambda)}{6} \tilde{u}_{2}^{3}+\frac{q_{003}(\lambda)}{6} \tilde{u}_{3}^{3}+\frac{q_{012}(\lambda)}{2} \tilde{u}_{2} \tilde{u}_{3}^{2}+ \\
& +q_{111}(\lambda) \tilde{u}_{1} \tilde{u}_{2} \tilde{u}_{3}+\frac{q_{021}(\lambda)}{2} \tilde{u}_{2}^{2} \tilde{u}_{3}+\frac{q_{102}(\lambda)}{2} \tilde{u}_{1} \tilde{u}_{3}^{2} \\
& +\frac{q_{201}(\lambda)}{2} \tilde{u}_{1}^{2} \tilde{u}_{3}+O(4)
\end{aligned}
$$

onde $\tilde{H}\left(\tilde{u}_{1}, \tilde{u}_{2}, \tilde{u}_{3}, 0\right)=h\left(\tilde{u}_{1}, \tilde{u}_{2}, \tilde{u}_{3}\right)$.

Observação 31 A fim de não sobrecarregar a notação, continuaremos usando $u_{1}, u_{2}$, $u_{3}$ ao invés de $\tilde{u}_{1}, \tilde{u}_{2}, \tilde{u}_{3}$.

Observação 32 Para o caso dos pontos $D_{1}^{1}, D_{2}^{1}, D_{3}^{1}, D_{13}^{1}, D_{1 h, n}^{1}$ e $D_{1 h, p}^{1}$, pode ser assumido que $a_{110}(\lambda)=0, a_{101}(\lambda)=0, a_{011}(\lambda)=0$ e $a_{200}(\lambda)=a_{020}(\lambda)$ em $\tilde{H}$, ou seja, que a origem é um ponto parcialmente umbílico de $\alpha_{\lambda}$. Isto segue do fato de que o conjunto parcialmente umbílico que contém um destes pontos é uma curva regular.

\subsection{Diagrama de Bifurcação de um Ponto Parcial- mente Umbílico do tipo $D_{1}^{1}$}

Seja $p$ um ponto parcialmente umbílico do tipo $D_{1}^{1}$ de uma imersão $\alpha: \mathbb{M}^{3} \rightarrow \mathbb{R}^{4}$. Escrevemos $\alpha$ como em (6.1) com $h$ satisfazendo a condição $D_{1}^{1}$. Seja $\alpha_{\lambda}$ uma família 
Diagramas de bifurcação dos pontos $D_{1}^{1}, D_{2}^{1}, D_{3}^{1}, D_{13}^{1}, D_{1 h, n}^{1}, D_{1 h, p}^{1}, D_{p}^{1}$ e $D_{c}^{1}$

a um parâmetro de imersões tal que $\alpha_{0}=\alpha$. Como visto na observação 32 podemos escrever

$$
\alpha_{\lambda}\left(u_{1}, u_{2}, u_{3}\right)=\left(u_{1}, u_{2}, u_{3}, h_{\lambda}\left(u_{1}, u_{2}, u_{3}\right)\right)
$$

onde

$$
\begin{aligned}
h_{\lambda}\left(u_{1}, u_{2}, u_{3}, \lambda\right)= & \frac{k(\lambda)}{2}\left(u_{1}^{2}+u_{2}^{2}\right)+\frac{k_{3}(\lambda)}{2} u_{3}^{2}+\frac{a(\lambda)}{6} u_{1}^{3}+\frac{b(\lambda)}{2} u_{1} u_{2}^{2}+\frac{d(\lambda)}{6} u_{1}^{2} u_{2}+ \\
& +\frac{c(\lambda)}{6} u_{2}^{3}+\frac{q_{003}(\lambda)}{6} u_{3}^{3}+\frac{q_{012}(\lambda)}{2} u_{2} u_{3}^{2}+q_{111}(\lambda) u_{1} u_{2} u_{3}+ \\
& +\frac{q_{021}(\lambda)}{2} u_{2}^{2} u_{3}+\frac{q_{102}(\lambda)}{2} u_{1} u_{3}^{2}+\frac{q_{201}(\lambda)}{2} u_{1}^{2} u_{3}+\frac{A(\lambda)}{24} u_{1}^{4}+ \\
& +\frac{B(\lambda)}{6} u_{1}^{3} u_{2}+\frac{C(\lambda)}{4} u_{1}^{2} u_{2}^{2}+\frac{D(\lambda)}{6} u_{1} u_{2}^{3}+\frac{E(\lambda)}{24} u_{2}^{4}+\frac{Q_{004}(\lambda)}{24} u_{3}^{4}+ \\
& +\frac{Q_{013}(\lambda)}{6} u_{3}^{3} u_{2}+\frac{Q_{103}(\lambda)}{6} u_{3}^{3} u_{1}+\frac{Q_{022}(\lambda)}{4} u_{2}^{2} u_{3}^{2}+\frac{Q_{202}(\lambda)}{4} u_{1}^{2} u_{3}^{2}+ \\
& +\frac{Q_{112}(\lambda)}{2} u_{3}^{2} u_{1} u_{2}+\frac{Q_{031}(\lambda)}{6} u_{3} u_{2}^{3}+\frac{Q_{301}(\lambda)}{6} u_{1}^{3} u_{3}+ \\
& +\frac{Q_{121}(\lambda)}{2} u_{3} u_{2}^{2} u_{1}+\frac{Q_{211}(\lambda)}{2} u_{3} u_{2} u_{1}^{2}+O(5) .
\end{aligned}
$$


Diagramas de bifurcação dos pontos $D_{1}^{1}, D_{2}^{1}, D_{3}^{1}, D_{13}^{1}, D_{1 h, n}^{1}, D_{1 h, p}^{1}, D_{p}^{1}$ e $D_{c}^{1}$

Teorema 13 Seja 0 um ponto parcialmente umbilico do tipo $D_{1}^{1}$ de uma imersão $\alpha$ como em (6.1). O diagrama de bifurcação de um ponto deste tipo é como na figura 6.1.

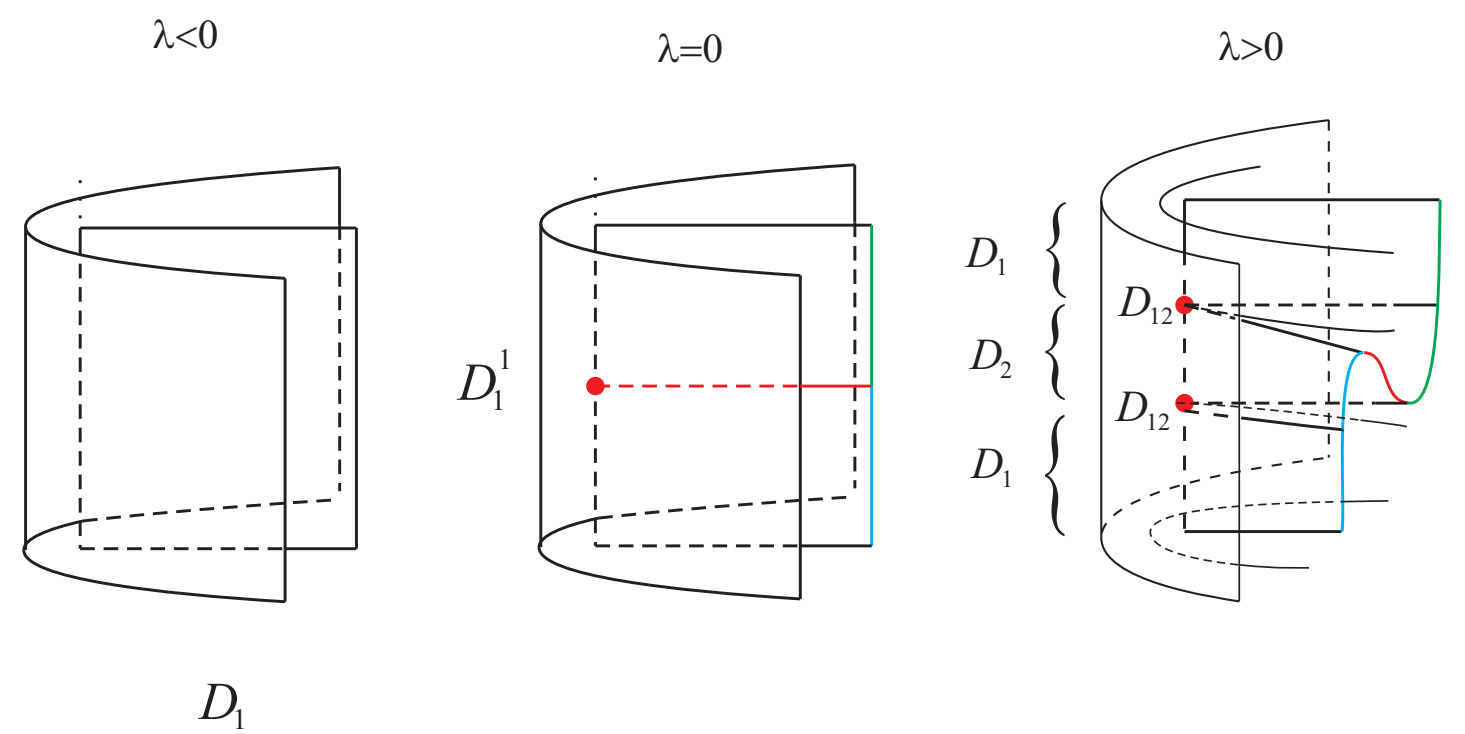

Figura 6.1: Bifurcação de uma curva parcialmente umbílica que contém um ponto do tipo $D_{1}^{1}$. 
Diagramas de bifurcação dos pontos $D_{1}^{1}, D_{2}^{1}, D_{3}^{1}, D_{13}^{1}, D_{1 h, n}^{1}, D_{1 h, p}^{1}, D_{p}^{1}$ e $D_{c}^{1}$

Demonstração: A equação diferencial das linhas de curvatura é

$$
\mathcal{L}\left(u_{1}, u_{2}, u_{3}, P, \lambda\right)=L_{r}\left(u_{1}, u_{2}, u_{3}, \lambda\right) P^{2}+M_{r}\left(u_{1}, u_{2}, u_{3}, \lambda\right) P+N_{r}\left(u_{1}, u_{2}, u_{3}, \lambda\right)
$$

onde

$$
\begin{aligned}
& L_{r}=-d(\lambda) u_{1}-b(\lambda) u_{2}-q_{111}(\lambda) u_{3}-\frac{\left(2 q_{201} q_{111}(\lambda)+B(\lambda) k(\lambda)-B k_{3}(\lambda)\right)}{2\left(k(\lambda)-k_{3}(\lambda)\right)} u_{1}^{2}- \\
& -\left(\frac{\left(2 q_{201} q_{021}+2 q_{111}^{2}\right)}{2\left(k(\lambda)-k_{3}(\lambda)\right)}+C(\lambda)-2 k(\lambda)^{3}\right) u_{1} u_{2}-\left(\frac{\left(2 q_{111}(\lambda) q_{021}(\lambda)\right)}{2\left(k(\lambda)-k_{3}(\lambda)\right)}+\right. \\
& \left.+\frac{D(\lambda)}{2}\right) u_{2}^{2}-\left(Q_{211}(\lambda)+\frac{\left(2 q_{102}(\lambda) q_{111}(\lambda)+2 q_{201}(\lambda) q_{012}(\lambda)\right)}{2\left(k(\lambda)-k_{3}(\lambda)\right)}\right) u_{1} u_{3}- \\
& -\left(Q_{121}(\lambda)+\frac{\left(q_{102}(\lambda) q_{021}(\lambda)+q_{111}(\lambda) q_{012}(\lambda)\right)}{\left(k(\lambda)-k_{3}(\lambda)\right)}\right) u_{2} u_{3}-\left(\frac{\left(q_{012}(\lambda) q_{102}(\lambda)\right)}{\left(k(\lambda)-k_{3}(\lambda)\right)}+\right. \\
& \left.+\frac{Q_{112}(\lambda)}{2}\right) u_{3}^{2}+O(3) \\
& M_{r}=(b(\lambda)-a(\lambda)) u_{1}+(c(\lambda)-d(\lambda)) u_{2}-\left(q_{201}(\lambda)-q_{021}(\lambda)\right) u_{3}+ \\
& +\left(-\frac{A(\lambda)}{2}+\frac{C(\lambda)}{2}+k(\lambda)^{3}-\frac{\left(q_{201}(\lambda)\right)^{2}-\left(q_{111}(\lambda)\right)^{2}}{k(\lambda)-k_{3}(\lambda)}\right) u_{1}^{2}+ \\
& +\left(-B(\lambda)-D(\lambda)-\frac{2 q_{201}(\lambda) q_{111}-2 q_{111}(\lambda) q_{021}(\lambda)}{k(\lambda)-k_{3}(\lambda)}\right) u_{1} u_{2}+ \\
& +\left(-\frac{-2 q_{111}(\lambda) q_{012}(\lambda)+2 q_{201}(\lambda) q_{102}(\lambda)}{k(\lambda)-k_{3}(\lambda)}+Q_{121}(\lambda)-Q_{301}(\lambda)\right) u_{1} u_{3}+ \\
& \left(\frac{E(\lambda)}{2}-\frac{C(\lambda)}{2}-k(\lambda)^{3}-\frac{\left(q_{111}(\lambda)\right)^{2}-\left(q_{021}(\lambda)\right)^{2}}{k(\lambda)-k_{3}(\lambda)}\right) u_{2}^{2}+ \\
& \left(-Q_{211}(\lambda)+Q_{031}(\lambda)-\frac{-2 q_{021}(\lambda) q_{012}(\lambda)+2 q_{102}(\lambda) q_{111}(\lambda)}{k(\lambda)-k_{3}(\lambda)}\right) u_{2} u_{3}+ \\
& +\left(\frac{Q_{022}(\lambda)}{2}-\frac{Q_{202}(\lambda)}{2}-\frac{-\left(q_{012}(\lambda)\right)^{2}+\left(q_{102}(\lambda)\right)^{2}}{k(\lambda)-k_{3}(\lambda)}\right) u_{3}^{2}+O(3)
\end{aligned}
$$


Diagramas de bifurcação dos pontos $D_{1}^{1}, D_{2}^{1}, D_{3}^{1}, D_{13}^{1}, D_{1 h, n}^{1}, D_{1 h, p}^{1}, D_{p}^{1}$ e $D_{c}^{1}$

$$
\begin{aligned}
N_{r} & =d(\lambda) u_{1}+b(\lambda) u_{2}+q_{111}(\lambda) u_{3}+\frac{\left(2 q_{201} q_{111}(\lambda)+B(\lambda) k(\lambda)-B k_{3}(\lambda)\right)}{2\left(k(\lambda)-k_{3}(\lambda)\right)} u_{1}^{2}+ \\
& +\left(\frac{\left(2 q_{201} q_{021}+2 q_{111}^{2}\right)}{2\left(k(\lambda)-k_{3}(\lambda)\right)}+C(\lambda)-2 k(\lambda)^{3}\right) u_{1} u_{2}+\left(\frac{\left(2 q_{111}(\lambda) q_{021}(\lambda)\right)}{2\left(k(\lambda)-k_{3}(\lambda)\right)}+\right. \\
& \left.+\frac{D(\lambda)}{2}\right) u_{2}^{2}+\left(Q_{211}(\lambda)+\frac{\left(2 q_{102}(\lambda) q_{111}(\lambda)+2 q_{201}(\lambda) q_{012}(\lambda)\right)}{2\left(k(\lambda)-k_{3}(\lambda)\right)}\right) u_{1} u_{3}+ \\
& +\left(Q_{121}(\lambda)+\frac{\left(q_{102}(\lambda) q_{021}(\lambda)+q_{111}(\lambda) q_{012}(\lambda)\right)}{\left(k(\lambda)-k_{3}(\lambda)\right)}\right) u_{2} u_{3}+\left(\frac{\left(q_{012}(\lambda) q_{102}(\lambda)\right)}{\left(k(\lambda)-k_{3}(\lambda)\right)}+\right. \\
& \left.+\frac{Q_{112}(\lambda)}{2}\right) u_{3}^{2}+O(3)
\end{aligned}
$$

Como vimos na seção 1.2 do capítulo 1, o campo de Lie-Cartan é dado por

$$
X:=\left\{\begin{array}{l}
\dot{u}_{1}=\mathcal{L}_{P} \\
\dot{u}_{2}=P \mathcal{L}_{P} \\
\dot{u}_{3}=(\mathcal{U}+\mathcal{V} P) \mathcal{L}_{P} \\
\dot{P}=-\left(\mathcal{L}_{u_{1}}+P \mathcal{L}_{u_{2}}+\mathcal{L}_{u_{3}}(\mathcal{U}+\mathcal{V} P)\right)
\end{array}\right.
$$

cujos equilíbrios, que se projetam nos pontos parcialmente umbílicos, satisfazem

$$
\left\{\begin{array}{l}
\left\{\begin{array}{l}
L_{r}\left(u_{1}, u_{2}, u_{3}\right)=0, \\
M_{r}\left(u_{1}, u_{2}, u_{3}\right)=0,
\end{array}\right. \\
\left(\mathcal{L}_{u_{1}}+P \mathcal{L}_{u_{2}}+\mathcal{L}_{u_{3}}(\mathcal{U}+\mathcal{V} P)\right)\left(u_{1}, u_{2}, u_{3}, P, \lambda\right)=0 .
\end{array}\right.
$$

A primeira equação de (6.8) define o conjunto parcialmente umbílico que neste caso pode ser escrito da forma: $u_{1}=u_{1}\left(u_{3}, \lambda\right), u_{2}=u_{2}\left(u_{3}, \lambda\right)$ já que, na carta de Monge reduzida,

$$
\operatorname{det}\left(\left.\frac{\partial\left(L_{r}, M_{r}\right)}{\partial\left(u_{1}, u_{2}\right)}\right|_{u_{1}=0, u_{2}=0, u_{3}=0, \lambda=0}\right)=-b^{2}
$$

Seja

$$
\mathcal{A}\left(u_{3}, P, \lambda\right)=\left(\mathcal{L}_{u_{1}}+P \mathcal{L}_{u_{2}}+\mathcal{L}_{u_{3}}(\mathcal{U}+\mathcal{V} P)\right)\left(u_{1}\left(u_{3}, \lambda\right), u_{2}\left(u_{3}, \lambda\right), u_{3}, P, \lambda\right) .
$$

Assim, as singularidades do campo de Lie-Cartan são dadas pelas raízes de um polinômio cúbico em $P$ :

$$
\mathcal{A}\left(u_{3}, P, \lambda\right)=\mathcal{A}_{3}\left(u_{3}, \lambda\right) P^{3}+\mathcal{A}_{2}\left(u_{3}, \lambda\right) P^{2}+\mathcal{A}_{1}\left(u_{3}, \lambda\right) P+\mathcal{A}_{0}\left(u_{3}, \lambda\right)
$$


Diagramas de bifurcação dos pontos $D_{1}^{1}, D_{2}^{1}, D_{3}^{1}, D_{13}^{1}, D_{1 h, n}^{1}, D_{1 h, p}^{1}, D_{p}^{1}$ e $D_{c}^{1}$ onde $\mathcal{A}(0, P, 0)=b P^{3}$ e $b \neq 0$.

Fazendo a mudança de coordenadas

$$
P=\tilde{P}-\frac{\mathcal{A}_{2}}{3 \mathcal{A}_{3}}
$$

obtemos

$$
\mathcal{B}\left(\lambda, u_{3}, \tilde{P}\right)=\mathcal{A}_{3}\left(u_{3}, \lambda\right) \tilde{P}^{3}+\mathcal{B}_{1}\left(u_{3}, \lambda\right) \tilde{P}+\mathcal{B}_{0}\left(u_{3}, \lambda\right)
$$

onde

$$
\mathcal{B}_{1}=\frac{27 \mathcal{A}_{1} \mathcal{A}_{3}^{2}-9 \mathcal{A}_{1} \mathcal{A}_{2}^{2}}{27 \mathcal{A}_{1}^{2}} \text { e } \mathcal{B}_{0}=\frac{-9 \mathcal{A}_{1} \mathcal{A}_{2} \mathcal{A}_{3}+27 \mathcal{A}_{0} \mathcal{A}_{1}^{2}+2 \mathcal{A}_{2}^{3}}{27 \mathcal{A}_{1}^{2}} \text {. }
$$

Seja $d(\lambda)=0, a(\lambda)=2 b+\lambda, b(\lambda)=b$. Desenvolvendo as contas, vemos que

$$
\left.\operatorname{det}\left(\frac{\partial\left(\mathcal{B}_{1}, \mathcal{B}_{0}\right)}{\partial\left(u_{3}, \lambda\right)}\right)\right|_{u_{3}=0=\lambda}=\frac{b \tilde{\chi}_{1}}{(k-1)} \neq 0
$$

Assim, os equilíbrios de $X$ formam uma variedade cuja projeção na variedade de pontos parcialmente umbílicos da família $\alpha_{\lambda}$, é como dado na figura 6.2. 
Diagramas de bifurcação dos pontos $D_{1}^{1}, D_{2}^{1}, D_{3}^{1}, D_{13}^{1}, D_{1 h, n}^{1}, D_{1 h, p}^{1}, D_{p}^{1}$ e $D_{c}^{1}$

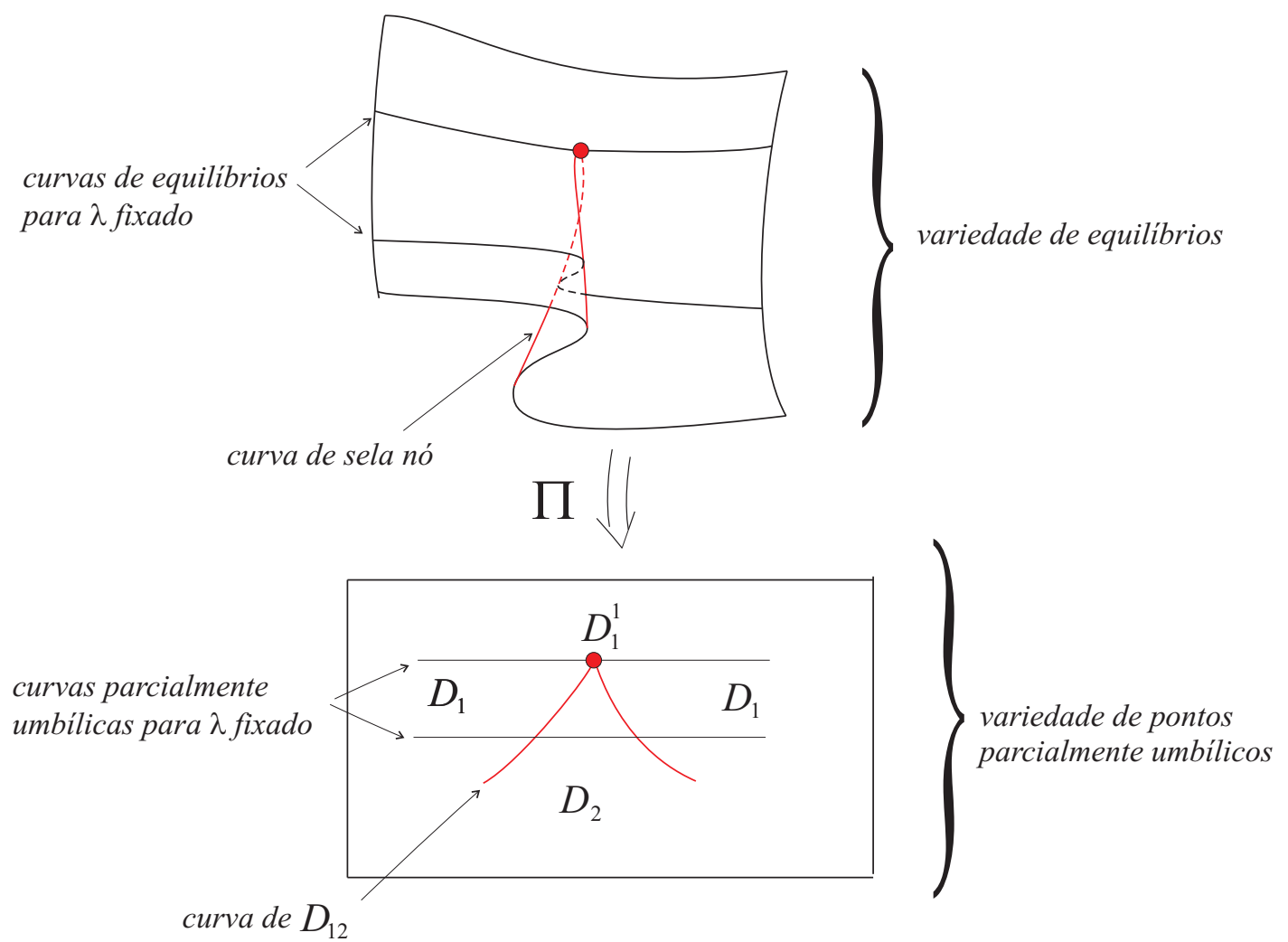

Figura 6.2: Resolução dos pontos parcialmente umbílicos de numa família a um parâmetro de hipersuperfícies, para o caso $D_{1}^{1}$.

Logo, a figura 6.1 mostra o diagrama de bifurcação para um ponto parcialmente umbílico do tipo $D_{1}^{1}$. Além disso, concluímos que o desdobramento transversal de $\alpha$ é equivalente à

$$
\alpha_{\lambda}\left(u_{1}, u_{2}, u_{3}\right)=\left(u_{1}, u_{2}, u_{3}, h\left(u_{1}, u_{2}, u_{3}\right)+\frac{\lambda}{6} u_{1}^{3}\right)
$$


Diagramas de bifurcação dos pontos $D_{1}^{1}, D_{2}^{1}, D_{3}^{1}, D_{13}^{1}, D_{1 h, n}^{1}, D_{1 h, p}^{1}, D_{p}^{1}$ e $D_{c}^{1}$

\subsection{Diagramas de Bifurcação dos Pontos Parcialmente Umbílicos $D_{2}^{1}$ e $D_{3}^{1}$}

Seja $p$ um ponto parcialmente umbílico do tipo $D_{2}^{1}$ ou $D_{3}^{1}$ de uma imersão $\alpha: \mathbb{M}^{3} \rightarrow \mathbb{R}^{4}$. Escrevemos $\alpha$ como em (6.1) com $h$ satisfazendo a condição $D_{2}^{1}$ ou $D_{3}^{1}$. Seja $\alpha_{\lambda}$ uma família a um parâmetro de imersões tal que $\alpha_{0}=\alpha$. Como visto na observação 32 podemos escrever

$$
\alpha_{\lambda}\left(u_{1}, u_{2}, u_{3}\right)=\left(u_{1}, u_{2}, u_{3}, h_{\lambda}\left(u_{1}, u_{2}, u_{3}\right)\right)
$$

onde

$$
\begin{aligned}
h_{\lambda}\left(u_{1}, u_{2}, u_{3}, \lambda\right)= & \frac{k(\lambda)}{2}\left(u_{1}^{2}+u_{2}^{2}\right)+\frac{k_{3}(\lambda)}{2} u_{3}^{2}+\frac{a(\lambda)}{6} u_{1}^{3}+\frac{b(\lambda)}{2} u_{1} u_{2}^{2}+\frac{d(\lambda)}{6} u_{1}^{2} u_{2}+ \\
& +\frac{c(\lambda)}{6} u_{2}^{3}+\frac{q_{003}(\lambda)}{6} u_{3}^{3}+\frac{q_{012}(\lambda)}{2} u_{2} u_{3}^{2}+q_{111}(\lambda) u_{1} u_{2} u_{3}+ \\
& +\frac{q_{021}(\lambda)}{2} u_{2}^{2} u_{3}+\frac{q_{102}(\lambda)}{2} u_{1} u_{3}^{2}+\frac{q_{201}(\lambda)}{2} u_{1}^{2} u_{3}+\frac{A(\lambda)}{24} u_{1}^{4}+ \\
& +\frac{B(\lambda)}{6} u_{1}^{3} u_{2}+\frac{C(\lambda)}{4} u_{1}^{2} u_{2}^{2}+\frac{D(\lambda)}{6} u_{1} u_{2}^{3}+\frac{E(\lambda)}{24} u_{2}^{4}+\frac{Q_{004}(\lambda)}{24} u_{3}^{4}+ \\
& +\frac{Q_{013}(\lambda)}{6} u_{3}^{3} u_{2}+\frac{Q_{103}(\lambda)}{6} u_{3}^{3} u_{1}+\frac{Q_{022}(\lambda)}{4} u_{2}^{2} u_{3}^{2}+\frac{Q_{202}(\lambda)}{4} u_{1}^{2} u_{3}^{2}+ \\
& +\frac{Q_{112}(\lambda)}{2} u_{3}^{2} u_{1} u_{2}+\frac{Q_{031}(\lambda)}{6} u_{3} u_{2}^{3}+\frac{Q_{301}(\lambda)}{6} u_{1}^{3} u_{3}+ \\
& +\frac{Q_{121}(\lambda)}{2} u_{3} u_{2}^{2} u_{1}+\frac{Q_{211}(\lambda)}{2} u_{3} u_{2} u_{1}^{2}+ \\
& +\sum_{i+j+k=5} \frac{a_{i j k}(\lambda)}{i ! j ! k !} u_{1}^{i} u_{2}^{j} u_{3}^{k}+O(6)
\end{aligned}
$$


Diagramas de bifurcação dos pontos $D_{1}^{1}, D_{2}^{1}, D_{3}^{1}, D_{13}^{1}, D_{1 h, n}^{1}, D_{1 h, p}^{1}, D_{p}^{1}$ e $D_{c}^{1}$

Teorema 14 Seja 0 um ponto parcialmente umbilico do tipo $D_{2}^{1}$ de uma imersão $\alpha$ como em (6.1). O diagrama de bifurcação de um ponto deste tipo é como na figura 6.3.

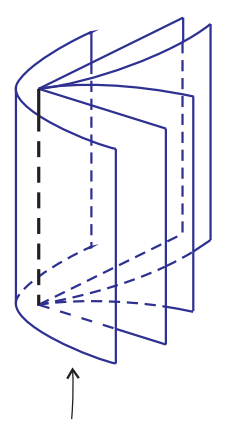

$D_{2}$

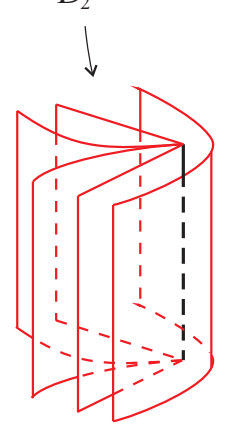

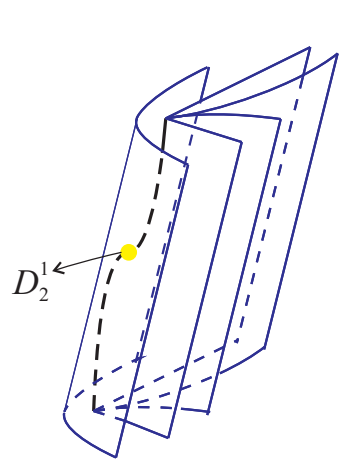

$D_{23}$

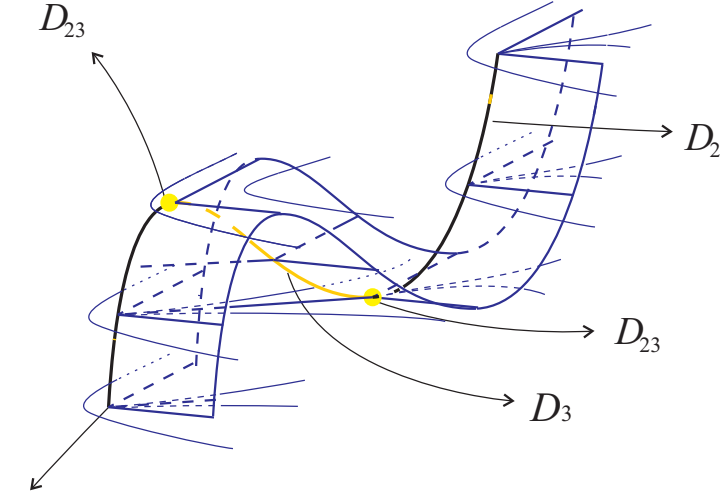

$D_{2}$
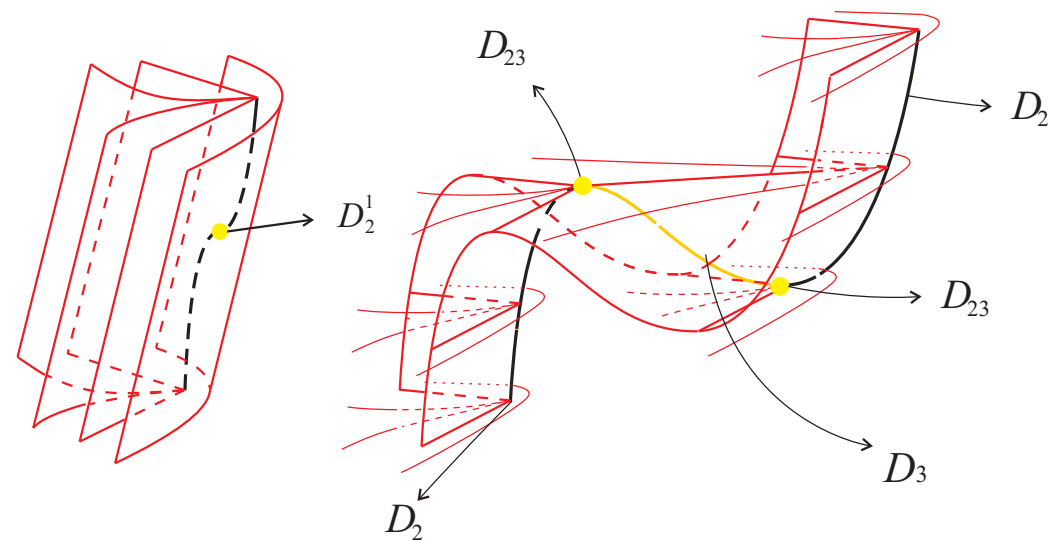

Figura 6.3: Bifurcação de uma linha parcialmente umbílica que contém um ponto do tipo $D_{2}^{1}(\tilde{\chi}<0)$. 
Diagramas de bifurcação dos pontos $D_{1}^{1}, D_{2}^{1}, D_{3}^{1}, D_{13}^{1}, D_{1 h, n}^{1}, D_{1 h, p}^{1}, D_{p}^{1}$ e $D_{c}^{1}$

Teorema 15 Seja 0 um ponto parcialmente umbílico do tipo $D_{3}^{1}$ de uma imersão $\alpha$ como em (6.1). O diagrama de bifurcação de um ponto deste tipo é como na figura 6.4.

$\lambda<0$

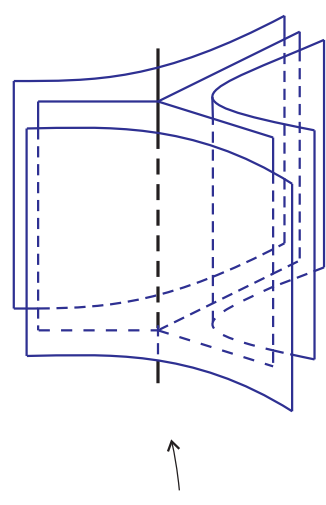

$D_{3}$

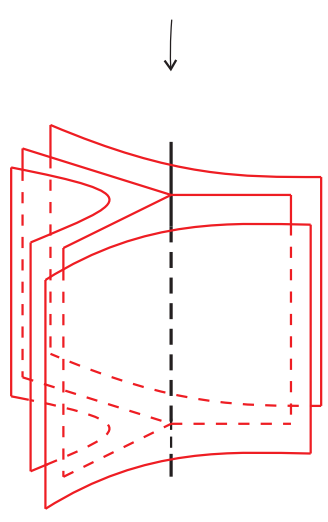

$\lambda=0$

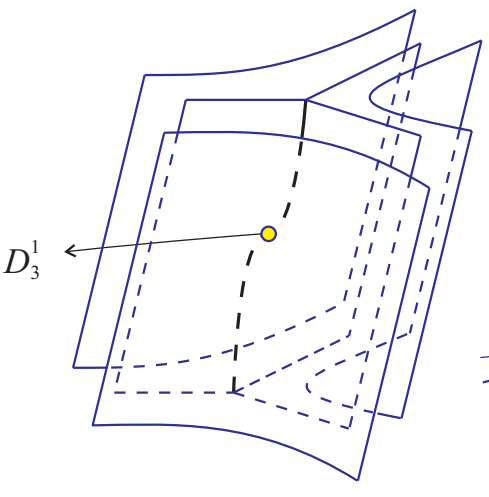

\section{$=$}

$D_{3}$

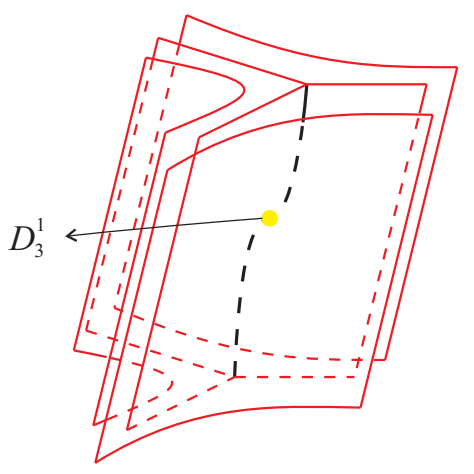

$\lambda>0$
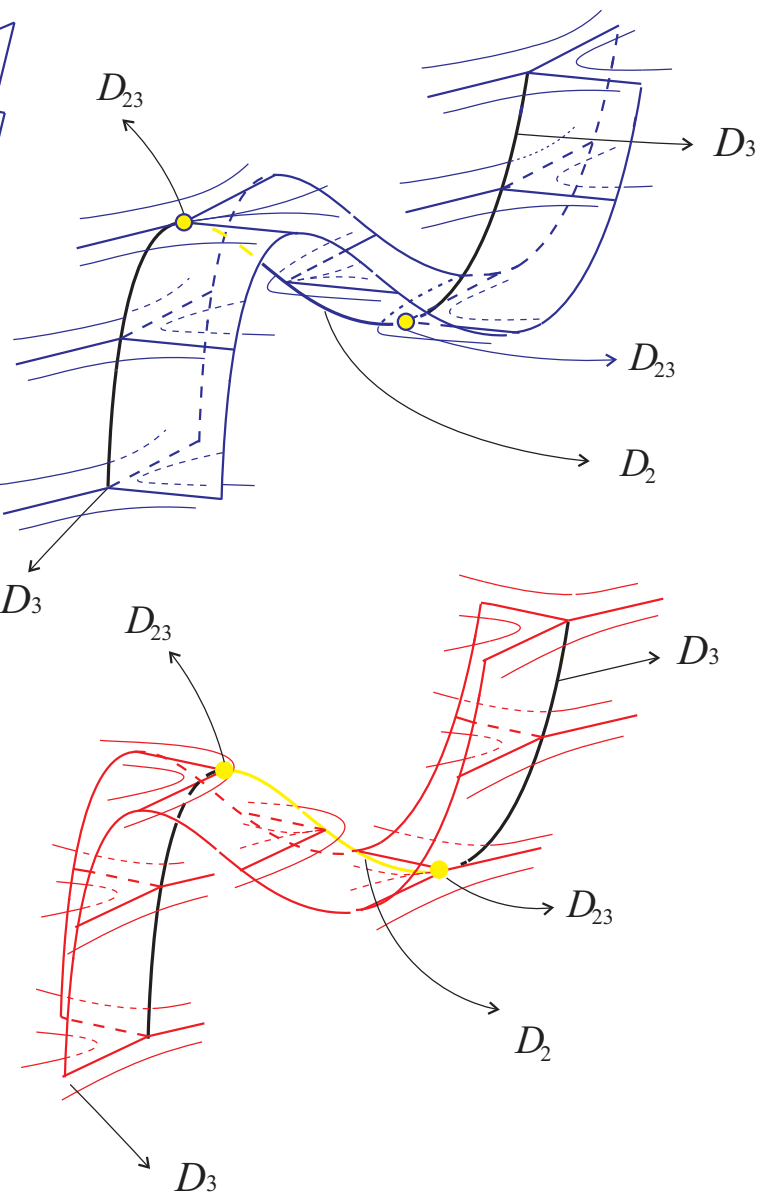

Figura 6.4: Bifurcação de uma linha parcialmente umbílica que contém um ponto do tipo $D_{3}^{1}(\tilde{\chi}>0)$. 
Diagramas de bifurcação dos pontos $D_{1}^{1}, D_{2}^{1}, D_{3}^{1}, D_{13}^{1}, D_{1 h, n}^{1}, D_{1 h, p}^{1}, D_{p}^{1}$ e $D_{c}^{1}$

Demonstração: A equação diferencial das linhas de curvatura é dada por

$$
\mathcal{L}\left(u_{1}, u_{2}, u_{3}, P, \lambda\right)=L_{r}\left(u_{1}, u_{2}, u_{3}, \lambda\right) P^{2}+M_{r}\left(u_{1}, u_{2}, u_{3}, \lambda\right) P+N_{r}\left(u_{1}, u_{2}, u_{3}, \lambda\right)
$$

onde

$$
\begin{aligned}
& L_{r}=-d(\lambda) u_{1}-b(\lambda) u_{2}-q_{111}(\lambda) u_{3}-\frac{\left(2 q_{201} q_{111}(\lambda)+B(\lambda) k(\lambda)-B k_{3}(\lambda)\right)}{2\left(k(\lambda)-k_{3}(\lambda)\right)} u_{1}^{2}- \\
& -\left(\frac{\left(2 q_{201} q_{021}+2 q_{111}^{2}\right)}{2\left(k(\lambda)-k_{3}(\lambda)\right)}+C(\lambda)-2 k(\lambda)^{3}\right) u_{1} u_{2}-\left(\frac{\left(2 q_{111}(\lambda) q_{021}(\lambda)\right)}{2\left(k(\lambda)-k_{3}(\lambda)\right)}+\right. \\
& \left.+\frac{D(\lambda)}{2}\right) u_{2}^{2}-\left(Q_{211}(\lambda)+\frac{\left(2 q_{102}(\lambda) q_{111}(\lambda)+2 q_{201}(\lambda) q_{012}(\lambda)\right)}{2\left(k(\lambda)-k_{3}(\lambda)\right)}\right) u_{1} u_{3}- \\
& -\left(Q_{121}(\lambda)+\frac{\left(q_{102}(\lambda) q_{021}(\lambda)+q_{111}(\lambda) q_{012}(\lambda)\right)}{\left(k(\lambda)-k_{3}(\lambda)\right)}\right) u_{2} u_{3}-\left(\frac{\left(q_{012}(\lambda) q_{102}(\lambda)\right)}{\left(k(\lambda)-k_{3}(\lambda)\right)}+\right. \\
& \left.+\frac{Q_{112}(\lambda)}{2}\right) u_{3}^{2}+O(3) \\
& M_{r}=(b(\lambda)-a(\lambda)) u_{1}+(c(\lambda)-d(\lambda)) u_{2}-\left(q_{201}(\lambda)-q_{021}(\lambda)\right) u_{3}+ \\
& +\left(-\frac{A(\lambda)}{2}+\frac{C(\lambda)}{2}+k(\lambda)^{3}-\frac{\left(q_{201}(\lambda)\right)^{2}-\left(q_{111}(\lambda)\right)^{2}}{k(\lambda)-k_{3}(\lambda)}\right) u_{1}^{2}+ \\
& +\left(-B(\lambda)-D(\lambda)-\frac{2 q_{201}(\lambda) q_{111}-2 q_{111}(\lambda) q_{021}(\lambda)}{k(\lambda)-k_{3}(\lambda)}\right) u_{1} u_{2}+ \\
& +\left(-\frac{-2 q_{111}(\lambda) q_{012}(\lambda)+2 q_{201}(\lambda) q_{102}(\lambda)}{k(\lambda)-k_{3}(\lambda)}+Q_{121}(\lambda)-Q_{301}(\lambda)\right) u_{1} u_{3}+ \\
& \left(\frac{E(\lambda)}{2}-\frac{C(\lambda)}{2}-k(\lambda)^{3}-\frac{\left(q_{111}(\lambda)\right)^{2}-\left(q_{021}(\lambda)\right)^{2}}{k(\lambda)-k_{3}(\lambda)}\right) u_{2}^{2}+ \\
& \left(-Q_{211}(\lambda)+Q_{031}(\lambda)-\frac{-2 q_{021}(\lambda) q_{012}(\lambda)+2 q_{102}(\lambda) q_{111}(\lambda)}{k(\lambda)-k_{3}(\lambda)}\right) u_{2} u_{3}+ \\
& +\left(\frac{Q_{022}(\lambda)}{2}-\frac{Q_{202}(\lambda)}{2}-\frac{-\left(q_{012}(\lambda)\right)^{2}+\left(q_{102}(\lambda)\right)^{2}}{k(\lambda)-k_{3}(\lambda)}\right) u_{3}^{2}+O(3)
\end{aligned}
$$


Diagramas de bifurcação dos pontos $D_{1}^{1}, D_{2}^{1}, D_{3}^{1}, D_{13}^{1}, D_{1 h, n}^{1}, D_{1 h, p}^{1}, D_{p}^{1}$ e $D_{c}^{1}$

$$
\begin{aligned}
N_{r} & =d(\lambda) u_{1}+b(\lambda) u_{2}+q_{111}(\lambda) u_{3}+\frac{\left(2 q_{201} q_{111}(\lambda)+B(\lambda) k(\lambda)-B k_{3}(\lambda)\right)}{2\left(k(\lambda)-k_{3}(\lambda)\right)} u_{1}^{2}+ \\
& +\left(\frac{\left(2 q_{201} q_{021}+2 q_{111}^{2}\right)}{2\left(k(\lambda)-k_{3}(\lambda)\right)}+C(\lambda)-2 k(\lambda)^{3}\right) u_{1} u_{2}+\left(\frac{\left(2 q_{111}(\lambda) q_{021}(\lambda)\right)}{2\left(k(\lambda)-k_{3}(\lambda)\right)}+\right. \\
& \left.+\frac{D(\lambda)}{2}\right) u_{2}^{2}+\left(Q_{211}(\lambda)+\frac{\left(2 q_{102}(\lambda) q_{111}(\lambda)+2 q_{201}(\lambda) q_{012}(\lambda)\right)}{2\left(k(\lambda)-k_{3}(\lambda)\right)}\right) u_{1} u_{3}+ \\
& +\left(Q_{121}(\lambda)+\frac{\left(q_{102}(\lambda) q_{021}(\lambda)+q_{111}(\lambda) q_{012}(\lambda)\right)}{\left(k(\lambda)-k_{3}(\lambda)\right)}\right) u_{2} u_{3}+\left(\frac{\left(q_{012}(\lambda) q_{102}(\lambda)\right)}{\left(k(\lambda)-k_{3}(\lambda)\right)}+\right. \\
& \left.+\frac{Q_{112}(\lambda)}{2}\right) u_{3}^{2}+O(3)
\end{aligned}
$$

Os pontos parcialmente umbílicos da família $\alpha_{\lambda}$ formam uma subvariedade, em $\mathbb{M} \times \mathbb{R}$, diferenciável e de dimensão 2. De fato, o conjunto dos pontos parcialmente umbílicos são definidos por

$$
L_{r}\left(u_{1}, u_{2}, u_{3}, \lambda\right)=0, \quad M_{r}\left(u_{1}, u_{2}, u_{3}, \lambda\right)=0,
$$

e satisfaz

$$
\operatorname{det}\left(\left.\frac{\partial\left(L_{r}, M_{r}\right)}{\partial\left(u_{2}, u_{3}\right)}\right|_{u_{1}=0=u_{2}=u_{3}=\lambda}\right)=c q_{111}-a\left(q_{021}-q_{201}\right) \neq 0
$$

Seja

$$
\mathcal{D}=\frac{\partial L_{r}}{\partial u_{1}} \frac{\partial M_{r}}{\partial u_{2}}-\frac{\partial M_{r}}{\partial u_{1}} \frac{\partial L_{r}}{\partial u_{2}}
$$

Podemos particionar a variedade dos pontos parcialmente umbílicos, numa vizinhança do ponto do tipo $D_{2}^{1}$ ou $D_{3}^{1}$, como segue:

- Pontos parcialmente umbílicos Darbouxianos:

$$
\left\{\begin{array}{l}
L_{r}\left(u_{1}, u_{2}, u_{3}, \lambda\right)=0 \\
M_{r}\left(u_{1}, u_{2}, u_{3}, \lambda\right)=0 \\
\mathcal{D}(0,0,0,0) \neq 0
\end{array}\right.
$$

Neste caso os pontos parcialmente umbílicos Darbouxianos são dos tipos $D_{2}, D_{3}$. 
Diagramas de bifurcação dos pontos $D_{1}^{1}, D_{2}^{1}, D_{3}^{1}, D_{13}^{1}, D_{1 h, n}^{1}, D_{1 h, p}^{1}, D_{p}^{1}$ e $D_{c}^{1}$

- Pontos parcialmente umbílicos semi-Darbouxianos do tipo $D_{23}$ :

$$
\left\{\begin{array}{l}
L_{r}\left(u_{1}, u_{2}, u_{3}, \lambda\right)=0 \\
M_{r}\left(u_{1}, u_{2}, u_{3}, \lambda\right)=0 \\
\mathcal{D}\left(u_{1}, u_{2}, u_{3}, \lambda\right)=0
\end{array}\right.
$$

Observação $33 \mathrm{Na}$ variedade dos pontos parcialmente umbílicos da família $\alpha_{\lambda}$ não aparecem pontos do tipo $D_{12}$, pois

$$
\left.\frac{\partial X_{4}}{\partial P}\right|_{u_{1}=u_{2}=u_{3}=P=\lambda=0}=a(0)-2 b(0) \neq 0 .
$$

Para os pontos parcialmente umbílicos semi-Darbouxianos do tipo $D_{23}$, teremos que

$$
\left.\frac{\partial\left(L_{r}, M_{r}, \mathcal{D}\right)}{\partial\left(u_{2}, u_{3}, \lambda\right)}\right|_{u_{1}=u_{2}=u_{3}=\lambda=0}=\left(b q_{021}+c q_{111}\right)\left(-\frac{\partial d}{\partial \lambda}(0) c+\frac{\partial b}{\partial \lambda}(0) b-b \frac{\partial a}{\partial \lambda}(0)\right)
$$

donde vemos que, se $d(\lambda)=0, a(\lambda)=a$ e $b(\lambda)=a+\lambda$

$$
\frac{\partial d}{\partial \lambda}(0) c+\frac{\partial b}{\partial \lambda}(0) b-b \frac{\partial a}{\partial \lambda}(0) \neq 0
$$

implicando que o sistema (6.12) define uma curva regular de pontos parcialmente umbílico do tipo $D_{23}$.

Assim, os diagramas de bifurcação da família $\alpha_{\lambda}$ em (6.9) são dados: na figura 6.3 se $\alpha_{0}=\alpha$ satisfaz a condição $D_{2}^{1}$, e na figura 6.4 se $\alpha_{0}=\alpha$ satisfaz a condição $D_{2}^{1}$.

Além disso, o desdobramento transversal da $\alpha$ é equivalente à família

$$
\alpha\left(u_{1}, u_{2}, u_{3}, \lambda\right)=\left(u_{1}, u_{2}, u_{3}, h\left(u_{1}, u_{2}, u_{3}\right)+\frac{\lambda}{2} u_{1} u_{2}^{2}\right) .
$$


Diagramas de bifurcação dos pontos $D_{1}^{1}, D_{2}^{1}, D_{3}^{1}, D_{13}^{1}, D_{1 h, n}^{1}, D_{1 h, p}^{1}, D_{p}^{1}$ e $D_{c}^{1}$

curvas de pontos parcialmente umbílicos no nível $\lambda$.
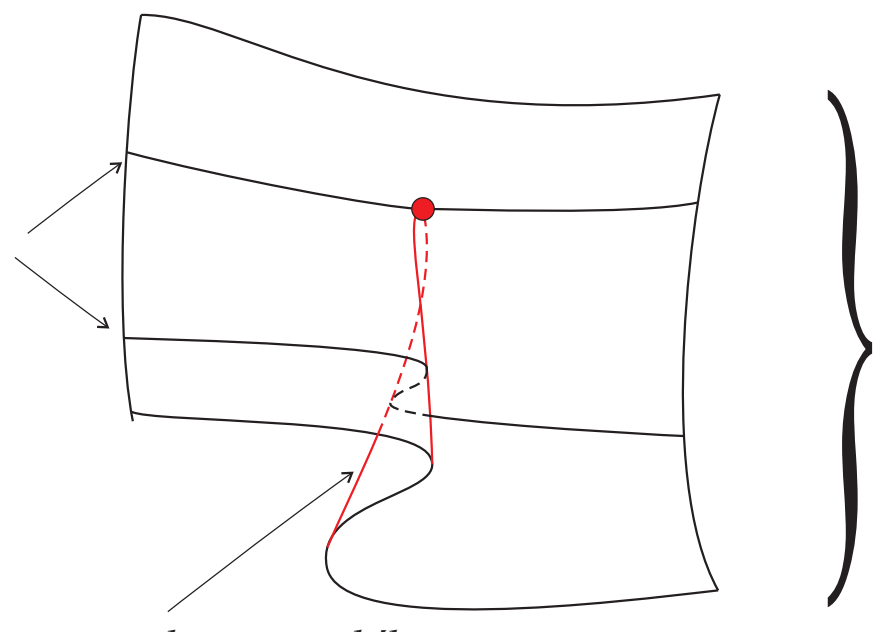

superficie parcialmente umbílica

\section{$D_{23}$ pontos parcialmente umbílicos}

(contato quadrático com o plano umbílico)

Figura 6.5: Conjunto parcialmente umbílico da família $\alpha_{\lambda}$ nas proximidades de um ponto do tipo $D_{c}^{1}$.

\subsection{Diagrama de Bifurcação de um Ponto Parcial- mente Umbílico do tipo $D_{13}^{1}$}

Seja $p$ um ponto parcialmente umbílico do tipo $D_{13}^{1}$ de uma imersão $\alpha: \mathbb{M}^{3} \rightarrow \mathbb{R}^{4}$. Escrevemos $\alpha$ como em (6.1) com $h$ satisfazendo a condição $D_{13}^{1}$. Seja $\alpha_{\lambda}$ uma família a um parâmetro de imersões tal que $\alpha_{0}=\alpha$. Como visto na observação 32 podemos escrever

$$
\alpha_{\lambda}\left(u_{1}, u_{2}, u_{3}\right)=\left(u_{1}, u_{2}, u_{3}, h_{\lambda}\left(u_{1}, u_{2}, u_{3}\right)\right)
$$


Diagramas de bifurcação dos pontos $D_{1}^{1}, D_{2}^{1}, D_{3}^{1}, D_{13}^{1}, D_{1 h, n}^{1}, D_{1 h, p}^{1}, D_{p}^{1}$ e $D_{c}^{1}$

onde

$$
\begin{aligned}
h_{\lambda}\left(u_{1}, u_{2}, u_{3}, \lambda\right)= & \frac{k(\lambda)}{2}\left(u_{1}^{2}+u_{2}^{2}\right)+\frac{k_{3}(\lambda)}{2} u_{3}^{2}+\frac{a(\lambda)}{6} u_{1}^{3}+\frac{b(\lambda)}{2} u_{1} u_{2}^{2}+\frac{d(\lambda)}{6} u_{1}^{2} u_{2}+ \\
& +\frac{c(\lambda)}{6} u_{2}^{3}+\frac{q_{003}(\lambda)}{6} u_{3}^{3}+\frac{q_{012}(\lambda)}{2} u_{2} u_{3}^{2}+q_{111}(\lambda) u_{1} u_{2} u_{3}+ \\
& +\frac{q_{021}(\lambda)}{2} u_{2}^{2} u_{3}+\frac{q_{102}(\lambda)}{2} u_{1} u_{3}^{2}+\frac{q_{201}(\lambda)}{2} u_{1}^{2} u_{3}+\frac{A(\lambda)}{24} u_{1}^{4}+ \\
& +\frac{B(\lambda)}{6} u_{1}^{3} u_{2}+\frac{C(\lambda)}{4} u_{1}^{2} u_{2}^{2}+\frac{D(\lambda)}{6} u_{1} u_{2}^{3}+\frac{E(\lambda)}{24} u_{2}^{4}+\frac{Q_{004}(\lambda)}{24} u_{3}^{4}+ \\
& +\frac{Q_{013}(\lambda)}{6} u_{3}^{3} u_{2}+\frac{Q_{103}(\lambda)}{6} u_{3}^{3} u_{1}+\frac{Q_{022}(\lambda)}{4} u_{2}^{2} u_{3}^{2}+\frac{Q_{202}(\lambda)}{4} u_{1}^{2} u_{3}^{2}+ \\
& +\frac{Q_{112}(\lambda)}{2} u_{3}^{2} u_{1} u_{2}+\frac{Q_{031}(\lambda)}{6} u_{3} u_{2}^{3}+\frac{Q_{301}(\lambda)}{6} u_{1}^{3} u_{3}+ \\
& +\frac{Q_{121}(\lambda)}{2} u_{3} u_{2}^{2} u_{1}+\frac{Q_{211}(\lambda)}{2} u_{3} u_{2} u_{1}^{2}+O(5)
\end{aligned}
$$


Diagramas de bifurcação dos pontos $D_{1}^{1}, D_{2}^{1}, D_{3}^{1}, D_{13}^{1}, D_{1 h, n}^{1}, D_{1 h, p}^{1}, D_{p}^{1}$ e $D_{c}^{1}$

Teorema 16 Seja 0 um ponto parcialmente umbílico do tipo $D_{13}^{1}$ de uma imersão $\alpha$ como em (6.1). O diagrama de bifurcação de um ponto deste tipo é como na figura 6.6.

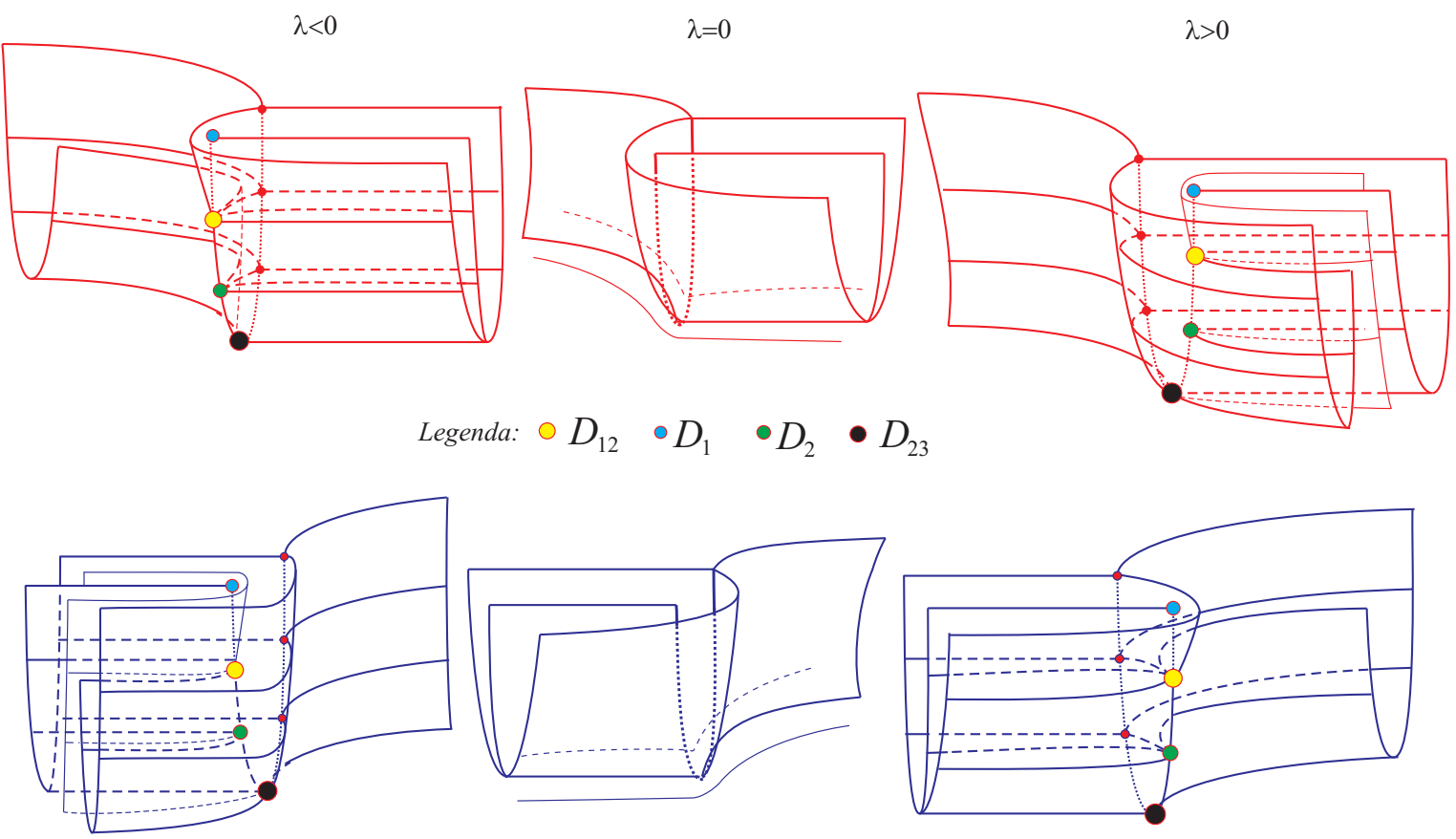

Figura 6.6: Bifurcação de uma linha parcialmente umbílica que contém um ponto do tipo $D_{13}^{1}$.

Demonstração: A equação diferencial das linhas de curvatura é dada por

$$
\mathcal{L}\left(u_{1}, u_{2}, u_{3}, P, \lambda\right)=L_{r}\left(u_{1}, u_{2}, u_{3}, \lambda\right) P^{2}+M_{r}\left(u_{1}, u_{2}, u_{3}, \lambda\right) P+N_{r}\left(u_{1}, u_{2}, u_{3}, \lambda\right)
$$

onde 
Diagramas de bifurcação dos pontos $D_{1}^{1}, D_{2}^{1}, D_{3}^{1}, D_{13}^{1}, D_{1 h, n}^{1}, D_{1 h, p}^{1}, D_{p}^{1}$ e $D_{c}^{1}$

$$
\begin{aligned}
& L_{r}=-d(\lambda) u_{1}-b(\lambda) u_{2}-q_{111}(\lambda) u_{3}-\frac{\left(2 q_{201} q_{111}(\lambda)+B(\lambda) k(\lambda)-B k_{3}(\lambda)\right)}{2\left(k(\lambda)-k_{3}(\lambda)\right)} u_{1}^{2}- \\
& -\left(\frac{\left(2 q_{201} q_{021}+2 q_{111}^{2}\right)}{2\left(k(\lambda)-k_{3}(\lambda)\right)}+C(\lambda)-2 k(\lambda)^{3}\right) u_{1} u_{2}-\left(\frac{\left(2 q_{111}(\lambda) q_{021}(\lambda)\right)}{2\left(k(\lambda)-k_{3}(\lambda)\right)}+\right. \\
& \left.+\frac{D(\lambda)}{2}\right) u_{2}^{2}-\left(Q_{211}(\lambda)+\frac{\left(2 q_{102}(\lambda) q_{111}(\lambda)+2 q_{201}(\lambda) q_{012}(\lambda)\right)}{2\left(k(\lambda)-k_{3}(\lambda)\right)}\right) u_{1} u_{3}- \\
& -\left(Q_{121}(\lambda)+\frac{\left(q_{102}(\lambda) q_{021}(\lambda)+q_{111}(\lambda) q_{012}(\lambda)\right)}{\left(k(\lambda)-k_{3}(\lambda)\right)}\right) u_{2} u_{3}-\left(\frac{\left(q_{012}(\lambda) q_{102}(\lambda)\right)}{\left(k(\lambda)-k_{3}(\lambda)\right)}+\right. \\
& \left.+\frac{Q_{112}(\lambda)}{2}\right) u_{3}^{2}+O(3) \\
& M_{r}=(b(\lambda)-a(\lambda)) u_{1}+(c(\lambda)-d(\lambda)) u_{2}-\left(q_{201}(\lambda)-q_{021}(\lambda)\right) u_{3}+ \\
& +\left(-\frac{A(\lambda)}{2}+\frac{C(\lambda)}{2}+k(\lambda)^{3}-\frac{\left(q_{201}(\lambda)\right)^{2}-\left(q_{111}(\lambda)\right)^{2}}{k(\lambda)-k_{3}(\lambda)}\right) u_{1}^{2}+ \\
& +\left(-B(\lambda)-D(\lambda)-\frac{2 q_{201}(\lambda) q_{111}-2 q_{111}(\lambda) q_{021}(\lambda)}{k(\lambda)-k_{3}(\lambda)}\right) u_{1} u_{2}+ \\
& +\left(-\frac{-2 q_{111}(\lambda) q_{012}(\lambda)+2 q_{201}(\lambda) q_{102}(\lambda)}{k(\lambda)-k_{3}(\lambda)}+Q_{121}(\lambda)-Q_{301}(\lambda)\right) u_{1} u_{3}+ \\
& \left(\frac{E(\lambda)}{2}-\frac{C(\lambda)}{2}-k(\lambda)^{3}-\frac{\left(q_{111}(\lambda)\right)^{2}-\left(q_{021}(\lambda)\right)^{2}}{k(\lambda)-k_{3}(\lambda)}\right) u_{2}^{2}+ \\
& \left(-Q_{211}(\lambda)+Q_{031}(\lambda)-\frac{-2 q_{021}(\lambda) q_{012}(\lambda)+2 q_{102}(\lambda) q_{111}(\lambda)}{k(\lambda)-k_{3}(\lambda)}\right) u_{2} u_{3}+ \\
& +\left(\frac{Q_{022}(\lambda)}{2}-\frac{Q_{202}(\lambda)}{2}-\frac{-\left(q_{012}(\lambda)\right)^{2}+\left(q_{102}(\lambda)\right)^{2}}{k(\lambda)-k_{3}(\lambda)}\right) u_{3}^{2}+O(3)
\end{aligned}
$$


Diagramas de bifurcação dos pontos $D_{1}^{1}, D_{2}^{1}, D_{3}^{1}, D_{13}^{1}, D_{1 h, n}^{1}, D_{1 h, p}^{1}, D_{p}^{1}$ e $D_{c}^{1}$

$$
\begin{aligned}
N_{r} & =d(\lambda) u_{1}+b(\lambda) u_{2}+q_{111}(\lambda) u_{3}+\frac{\left(2 q_{201} q_{111}(\lambda)+B(\lambda) k(\lambda)-B k_{3}(\lambda)\right)}{2\left(k(\lambda)-k_{3}(\lambda)\right)} u_{1}^{2}+ \\
& +\left(\frac{\left(2 q_{201} q_{021}+2 q_{111}^{2}\right)}{2\left(k(\lambda)-k_{3}(\lambda)\right)}+C(\lambda)-2 k(\lambda)^{3}\right) u_{1} u_{2}+\left(\frac{\left(2 q_{111}(\lambda) q_{021}(\lambda)\right)}{2\left(k(\lambda)-k_{3}(\lambda)\right)}+\right. \\
& \left.+\frac{D(\lambda)}{2}\right) u_{2}^{2}+\left(Q_{211}(\lambda)+\frac{\left(2 q_{102}(\lambda) q_{111}(\lambda)+2 q_{201}(\lambda) q_{012}(\lambda)\right)}{2\left(k(\lambda)-k_{3}(\lambda)\right)}\right) u_{1} u_{3}+ \\
& +\left(Q_{121}(\lambda)+\frac{\left(q_{102}(\lambda) q_{021}(\lambda)+q_{111}(\lambda) q_{012}(\lambda)\right)}{\left(k(\lambda)-k_{3}(\lambda)\right)}\right) u_{2} u_{3}+\left(\frac{\left(q_{012}(\lambda) q_{102}(\lambda)\right)}{\left(k(\lambda)-k_{3}(\lambda)\right)}+\right. \\
& \left.+\frac{Q_{112}(\lambda)}{2}\right) u_{3}^{2}+O(3)
\end{aligned}
$$

Seja

$$
\mathcal{D}=\frac{\partial L}{\partial u_{1}} \frac{\partial M}{\partial u_{2}}-\frac{\partial M}{\partial u_{1}} \frac{\partial L}{\partial u_{2}} .
$$

Pelo teorema da função implícita, o conjunto dos pontos que satisfaz

$$
\left\{\begin{array}{l}
L_{r}\left(u_{1}, u_{2}, u_{3}, \lambda\right)=0 \\
M_{r}\left(u_{1}, u_{2}, u_{3}, \lambda\right)=0 \\
\mathcal{D}\left(u_{1}, u_{2}, u_{3}, \lambda\right)=0
\end{array}\right.
$$

forma uma curva regular no espaço das variáveis $u_{1}, u_{2}, u_{3}$ e $\lambda$, já que

$$
\operatorname{det}\left(\left.\frac{\partial\left(L_{r}, M_{r}, \mathcal{D}\right)}{\partial\left(u_{1}, u_{2}, u_{3}\right)}\right|_{u_{1}=0=u_{2}=u_{3}=\lambda}\right)=-\frac{B k-B+3 q_{111} q_{201}}{k-1} \neq 0 .
$$

Tal curva é constituída de pontos parcialmente umbílicos do tipo $D_{23}$, veja seção 2.3 do capítulo 2 .

Além da curva de pontos parcialmente umbílicos do tipo $D_{23}$, a variedade de pontos parcialmente umbílicos da família $\alpha_{\lambda}$ contém uma curva de pontos do tipo $D_{12}$. De fato, denotemos a quarta componente do campo de Lie-Cartan por

$$
X_{4}\left(u_{1}, u_{2}, u_{3}, P, \lambda\right)=-\left(\mathcal{L}_{u_{1}}+P \mathcal{L}_{u_{2}}+\mathcal{L}_{u_{3}}(\mathcal{U}+\mathcal{V} P)\right)\left(u_{1}, u_{2}, u_{3}, P, \lambda\right)
$$

Os pontos parcialmente do tipo $D_{12}$ são definidos pelo sistema

$$
\left\{\begin{array}{l}
L_{r}\left(u_{1}, u_{2}, u_{3}, \lambda\right)=0 \\
M_{r}\left(u_{1}, u_{2}, u_{3}, \lambda\right)=0 \\
X_{4}\left(u_{1}, u_{2}, u_{3}, P, \lambda\right)=0 \\
\frac{\partial X_{4}}{\partial P}\left(u_{1}, u_{2}, u_{3}, P, \lambda\right)=0
\end{array} .\right.
$$


Diagramas de bifurcação dos pontos $D_{1}^{1}, D_{2}^{1}, D_{3}^{1}, D_{13}^{1}, D_{1 h, n}^{1}, D_{1 h, p}^{1}, D_{p}^{1}$ e $D_{c}^{1}$

Desenvolvendo os cálculos vemos que

$$
\begin{aligned}
& \left.\operatorname{det}\left(\frac{\partial\left(L_{r}, M_{r}, X_{4}, \frac{\partial X_{4}}{\partial u_{1}}\right)}{\partial\left(u_{1}, u_{2}, u_{3}, \lambda\right)}\right)\right|_{u_{1}=u_{2}=u_{3}=\lambda=P=0}= \\
& \frac{\left(3 q_{111} q_{201}+B k-B\right) c q_{111}}{k-1}\left(\frac{\partial a}{\partial \lambda}(0)-2 \frac{\partial b}{\partial \lambda}(0)\right)- \\
& -\frac{\left(-2 k^{4}+k^{3}+2 A k-4 C k-A-8 q_{111}^{2}+2 C-4 q_{021} q_{201}+6 q_{201}^{2}\right) c q_{111}}{k-1} \frac{\partial d}{\partial \lambda}(0)
\end{aligned}
$$

Logo, escolhendo adequadamente $d(\lambda), a(\lambda)$ e $b(\lambda)$, o sistema (6.19) define uma curva regular de pontos parcialmente umbílicos do tipo $D_{12}$.

Se $d(\lambda)=0, a(\lambda)=b(\lambda)=\lambda$ teremos que

$$
\left.\operatorname{det}\left(\frac{\partial\left(L_{r}, M_{r}, X_{4}, \frac{\partial X_{4}}{\partial P}\right)}{\partial\left(u_{1}, u_{2}, u_{3}, P\right)}\right)\right|_{u_{1}=u_{2}=u_{3}=\lambda=P=0}=2 c^{2} q_{111}\left(c B-\frac{3 q_{201} q_{111} c}{k-1}\right) \neq 0
$$

Neste caso, podemos escrever $u_{1}=u_{1}(\lambda), u_{2}=u_{2}(\lambda), u_{3}=u_{3}(\lambda)$ e $P=P(\lambda) \mathrm{em}$ (6.19). Nas variáveis $u_{3}$ e $\lambda$ teremos que

$$
u_{3}(\lambda)=-\frac{3}{4} \frac{(k-1)^{2} B+2(k-1) q_{201} q_{111}}{q_{111} c^{2}\left(B(k-1)+3 q_{201} q_{111}\right)^{2}} \lambda^{4}+O(5) .
$$

A figura 6.7 abaixo ilustra o contato das curvas de pontos $D_{12}$ e $D_{23}$ na variedade de pontos parcialmente umbílicos.

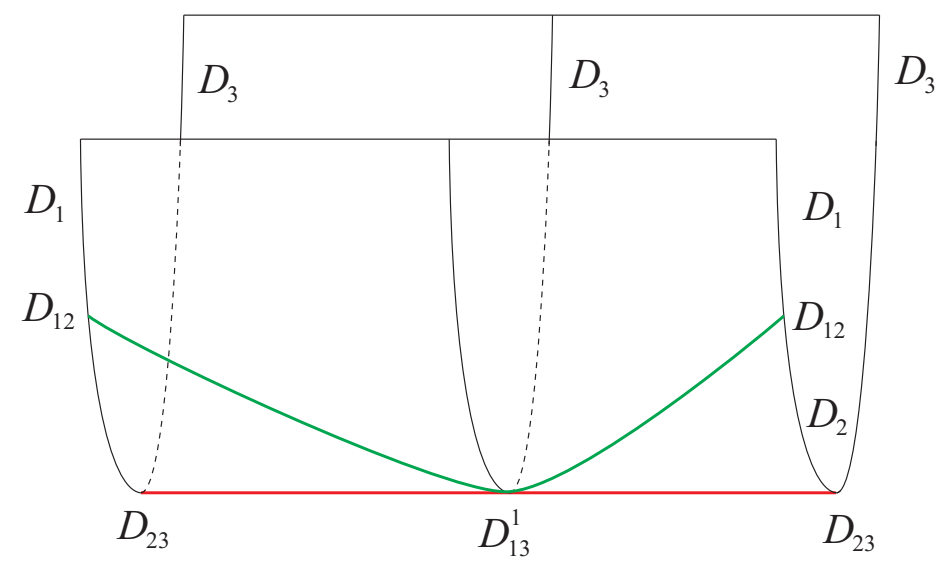

Figura 6.7: Conjunto parcialmente umbílico de uma família a 1 parâmetro associada a um ponto do tipo $D_{13}^{1}$. 
Diagramas de bifurcação dos pontos $D_{1}^{1}, D_{2}^{1}, D_{3}^{1}, D_{13}^{1}, D_{1 h, n}^{1}, D_{1 h, p}^{1}, D_{p}^{1}$ e $D_{c}^{1}$

Assim, concluímos que o diagrama de bifurcação de um ponto do tipo $D_{13}^{1}$ é como na figura 6.6. Além disso, o desdobramento transversal da $\alpha$ é equivalente à família

$$
\alpha\left(u_{1}, u_{2}, u_{3}, \lambda\right)=\left(u_{1}, u_{2}, u_{3}, h\left(u_{1}, u_{2}, u_{3}\right)+\frac{\lambda}{6}\left(u_{1}^{3}+3 u_{1} u_{2}^{2}\right)\right) .
$$


Diagramas de bifurcação dos pontos $D_{1}^{1}, D_{2}^{1}, D_{3}^{1}, D_{13}^{1}, D_{1 h, n}^{1}, D_{1 h, p}^{1}, D_{p}^{1}$ e $D_{c}^{1}$

\subsection{Diagramas de Bifurcação dos Pontos Parcialmente Umbílicos $D_{1 h, p}^{1}$ e $D_{1 h, n}^{1}$}

Seja $p$ um ponto parcialmente umbílico do tipo $D_{1 h, p}^{1}$ ou $D_{1 h, n}^{1}$ de uma imersão $\alpha$ : $\mathbb{M}^{3} \rightarrow \mathbb{R}^{4}$. Escrevemos $\alpha$ como em (6.1) com $k=0$ e $h$ satisfazendo a condição $D_{1 h, p}^{1}$ ou $D_{1 h, n}^{1}$. Seja $\alpha_{\lambda}$ uma família a um parâmetro de imersões tal que $\alpha_{0}=\alpha$. Como visto na observação 32 podemos escrever

$$
\alpha_{\lambda}\left(u_{1}, u_{2}, u_{3}\right)=\left(u_{1}, u_{2}, u_{3}, h_{\lambda}\left(u_{1}, u_{2}, u_{3}\right)\right)
$$

onde

$$
\begin{aligned}
h_{\lambda}\left(u_{1}, u_{2}, u_{3}, \lambda\right)= & \frac{k(\lambda)}{2}\left(u_{1}^{2}+u_{2}^{2}\right)+\frac{k_{3}(\lambda)}{2} u_{3}^{2}+\frac{a(\lambda)}{6} u_{1}^{3}+\frac{b(\lambda)}{2} u_{1} u_{2}^{2}+\frac{d(\lambda)}{6} u_{1}^{2} u_{2}+ \\
& +\frac{c(\lambda)}{6} u_{2}^{3}+\frac{q_{003}(\lambda)}{6} u_{3}^{3}+\frac{q_{012}(\lambda)}{2} u_{2} u_{3}^{2}+q_{111}(\lambda) u_{1} u_{2} u_{3}+ \\
& +\frac{q_{021}(\lambda)}{2} u_{2}^{2} u_{3}+\frac{q_{102}(\lambda)}{2} u_{1} u_{3}^{2}+\frac{q_{201}(\lambda)}{2} u_{1}^{2} u_{3}+\frac{A(\lambda)}{24} u_{1}^{4}+ \\
& +\frac{B(\lambda)}{6} u_{1}^{3} u_{2}+\frac{C(\lambda)}{4} u_{1}^{2} u_{2}^{2}+\frac{D(\lambda)}{6} u_{1} u_{2}^{3}+\frac{E(\lambda)}{24} u_{2}^{4}+\frac{Q_{004}(\lambda)}{24} u_{3}^{4}+ \\
& +\frac{Q_{013}(\lambda)}{6} u_{3}^{3} u_{2}+\frac{Q_{103}(\lambda)}{6} u_{3}^{3} u_{1}+\frac{Q_{022}(\lambda)}{4} u_{2}^{2} u_{3}^{2}+\frac{Q_{202}(\lambda)}{4} u_{1}^{2} u_{3}^{2}+ \\
& +\frac{Q_{112}(\lambda)}{2} u_{3}^{2} u_{1} u_{2}+\frac{Q_{031}(\lambda)}{6} u_{3} u_{2}^{3}+\frac{Q_{301}(\lambda)}{6} u_{1}^{3} u_{3}+ \\
& +\frac{Q_{121}(\lambda)}{2} u_{3} u_{2}^{2} u_{1}+\frac{Q_{211}(\lambda)}{2} u_{3} u_{2} u_{1}^{2}+O(5)
\end{aligned}
$$


Diagramas de bifurcação dos pontos $D_{1}^{1}, D_{2}^{1}, D_{3}^{1}, D_{13}^{1}, D_{1 h, n}^{1}, D_{1 h, p}^{1}, D_{p}^{1}$ e $D_{c}^{1}$

Teorema 17 Seja 0 um ponto parcialmente umbílico do tipo $D_{1 h, p}^{1}$ de uma imersão $\alpha$ como em (6.1). O diagrama de bifurcação de um ponto deste tipo é como na figura 6.8.

$\lambda<0$

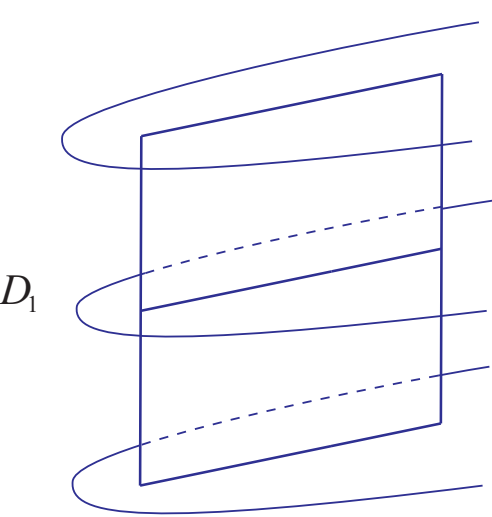

$\lambda=0$

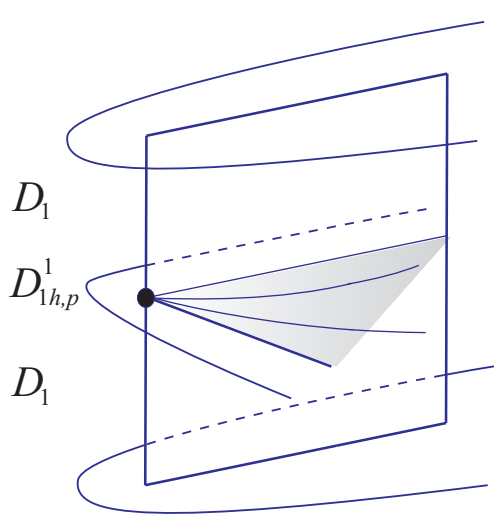

$\lambda>0$

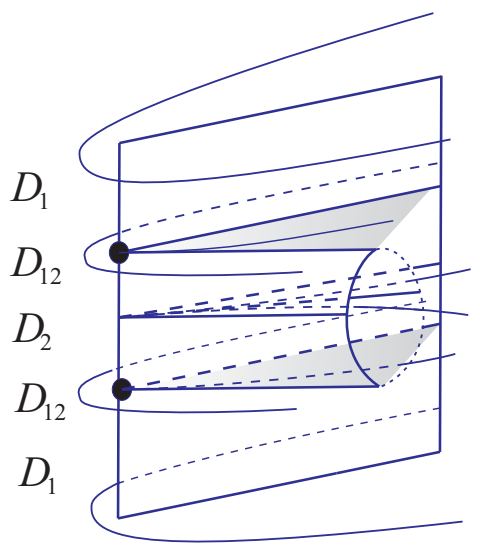

Figura 6.8: Bifurcação de uma linha parcialmente umbílica que contém um ponto do tipo $D_{1 h, p}^{1}$. 
Diagramas de bifurcação dos pontos $D_{1}^{1}, D_{2}^{1}, D_{3}^{1}, D_{13}^{1}, D_{1 h, n}^{1}, D_{1 h, p}^{1}, D_{p}^{1}$ e $D_{c}^{1}$

Teorema 18 Seja 0 um ponto parcialmente umbílico do tipo $D_{1 h, n}^{1}$ de uma imersão $\alpha$ como em (6.1). O diagrama de bifurcação de um ponto deste tipo é como na figura 6.9.

$\lambda<0$

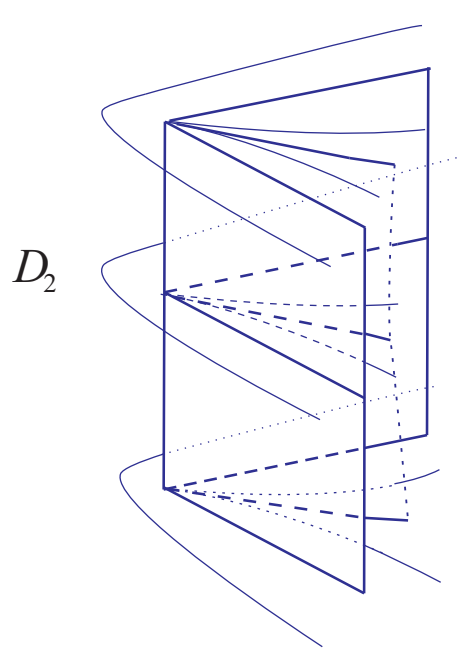

$\lambda=0$

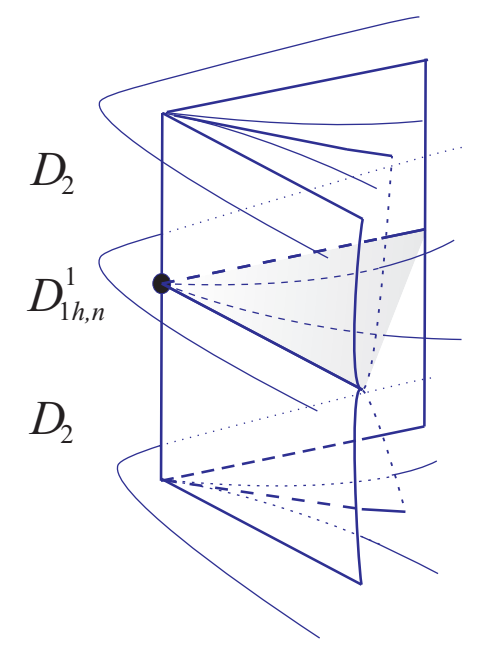

$\lambda>0$

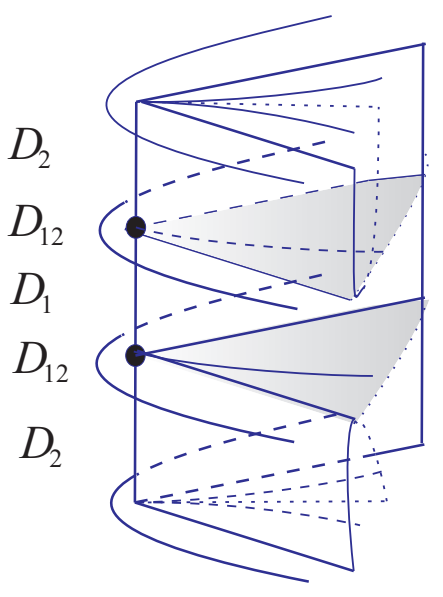

Figura 6.9: Bifurcação de uma linha parcialmente umbílica que contém um ponto do tipo $D_{1 h, n}^{1}$. 
Diagramas de bifurcação dos pontos $D_{1}^{1}, D_{2}^{1}, D_{3}^{1}, D_{13}^{1}, D_{1 h, n}^{1}, D_{1 h, p}^{1}, D_{p}^{1}$ e $D_{c}^{1}$

Demonstração dos teoremas 17 e 18: A equação diferencial das linhas de curvatura é dada por

$$
\mathcal{L}\left(u_{1}, u_{2}, u_{3}, P, \lambda\right)=L_{r}\left(u_{1}, u_{2}, u_{3}, \lambda\right) P^{2}+M_{r}\left(u_{1}, u_{2}, u_{3}, \lambda\right) P+N_{r}\left(u_{1}, u_{2}, u_{3}, \lambda\right)
$$

onde

$$
\begin{aligned}
& L_{r}=-d(\lambda) u_{1}-b(\lambda) u_{2}-q_{111}(\lambda) u_{3}-\frac{\left(2 q_{201} q_{111}(\lambda)+B(\lambda) k(\lambda)-B k_{3}(\lambda)\right)}{2\left(k(\lambda)-k_{3}(\lambda)\right)} u_{1}^{2}- \\
& -\left(\frac{\left(2 q_{201} q_{021}+2 q_{111}^{2}\right)}{2\left(k(\lambda)-k_{3}(\lambda)\right)}+C(\lambda)-2 k(\lambda)^{3}\right) u_{1} u_{2}-\left(\frac{\left(2 q_{111}(\lambda) q_{021}(\lambda)\right)}{2\left(k(\lambda)-k_{3}(\lambda)\right)}+\right. \\
& \left.+\frac{D(\lambda)}{2}\right) u_{2}^{2}-\left(Q_{211}(\lambda)+\frac{\left(2 q_{102}(\lambda) q_{111}(\lambda)+2 q_{201}(\lambda) q_{012}(\lambda)\right)}{2\left(k(\lambda)-k_{3}(\lambda)\right)}\right) u_{1} u_{3}- \\
& -\left(Q_{121}(\lambda)+\frac{\left(q_{102}(\lambda) q_{021}(\lambda)+q_{111}(\lambda) q_{012}(\lambda)\right)}{\left(k(\lambda)-k_{3}(\lambda)\right)}\right) u_{2} u_{3}-\left(\frac{\left(q_{012}(\lambda) q_{102}(\lambda)\right)}{\left(k(\lambda)-k_{3}(\lambda)\right)}+\right. \\
& \left.+\frac{Q_{112}(\lambda)}{2}\right) u_{3}^{2}+O(3) \\
& M_{r}=(b(\lambda)-a(\lambda)) u_{1}+(c(\lambda)-d(\lambda)) u_{2}-\left(q_{201}(\lambda)-q_{021}(\lambda)\right) u_{3}+ \\
& +\left(-\frac{A(\lambda)}{2}+\frac{C(\lambda)}{2}+k(\lambda)^{3}-\frac{\left(q_{201}(\lambda)\right)^{2}-\left(q_{111}(\lambda)\right)^{2}}{k(\lambda)-k_{3}(\lambda)}\right) u_{1}^{2}+ \\
& +\left(-B(\lambda)-D(\lambda)-\frac{2 q_{201}(\lambda) q_{111}-2 q_{111}(\lambda) q_{021}(\lambda)}{k(\lambda)-k_{3}(\lambda)}\right) u_{1} u_{2}+ \\
& +\left(-\frac{-2 q_{111}(\lambda) q_{012}(\lambda)+2 q_{201}(\lambda) q_{102}(\lambda)}{k(\lambda)-k_{3}(\lambda)}+Q_{121}(\lambda)-Q_{301}(\lambda)\right) u_{1} u_{3}+ \\
& \left(\frac{E(\lambda)}{2}-\frac{C(\lambda)}{2}-k(\lambda)^{3}-\frac{\left(q_{111}(\lambda)\right)^{2}-\left(q_{021}(\lambda)\right)^{2}}{k(\lambda)-k_{3}(\lambda)}\right) u_{2}^{2}+ \\
& \left(-Q_{211}(\lambda)+Q_{031}(\lambda)-\frac{-2 q_{021}(\lambda) q_{012}(\lambda)+2 q_{102}(\lambda) q_{111}(\lambda)}{k(\lambda)-k_{3}(\lambda)}\right) u_{2} u_{3}+ \\
& +\left(\frac{Q_{022}(\lambda)}{2}-\frac{Q_{202}(\lambda)}{2}-\frac{-\left(q_{012}(\lambda)\right)^{2}+\left(q_{102}(\lambda)\right)^{2}}{k(\lambda)-k_{3}(\lambda)}\right) u_{3}^{2}+O(3)
\end{aligned}
$$


Diagramas de bifurcação dos pontos $D_{1}^{1}, D_{2}^{1}, D_{3}^{1}, D_{13}^{1}, D_{1 h, n}^{1}, D_{1 h, p}^{1}, D_{p}^{1}$ e $D_{c}^{1}$

$$
\begin{aligned}
N_{r} & =d(\lambda) u_{1}+b(\lambda) u_{2}+q_{111}(\lambda) u_{3}+\frac{\left(2 q_{201} q_{111}(\lambda)+B(\lambda) k(\lambda)-B k_{3}(\lambda)\right)}{2\left(k(\lambda)-k_{3}(\lambda)\right)} u_{1}^{2}+ \\
& +\left(\frac{\left(2 q_{201} q_{021}+2 q_{111}^{2}\right)}{2\left(k(\lambda)-k_{3}(\lambda)\right)}+C(\lambda)-2 k(\lambda)^{3}\right) u_{1} u_{2}+\left(\frac{\left(2 q_{111}(\lambda) q_{021}(\lambda)\right)}{2\left(k(\lambda)-k_{3}(\lambda)\right)}+\right. \\
& \left.+\frac{D(\lambda)}{2}\right) u_{2}^{2}+\left(Q_{211}(\lambda)+\frac{\left(2 q_{102}(\lambda) q_{111}(\lambda)+2 q_{201}(\lambda) q_{012}(\lambda)\right)}{2\left(k(\lambda)-k_{3}(\lambda)\right)}\right) u_{1} u_{3}+ \\
& +\left(Q_{121}(\lambda)+\frac{\left(q_{102}(\lambda) q_{021}(\lambda)+q_{111}(\lambda) q_{012}(\lambda)\right)}{\left(k(\lambda)-k_{3}(\lambda)\right)}\right) u_{2} u_{3}+\left(\frac{\left(q_{012}(\lambda) q_{102}(\lambda)\right)}{\left(k(\lambda)-k_{3}(\lambda)\right)}+\right. \\
& \left.+\frac{Q_{112}(\lambda)}{2}\right) u_{3}^{2}+O(3)
\end{aligned}
$$

Denotando a quarta componente do campo de Lie-Cartan por

$$
X_{4}\left(u_{1}, u_{2}, u_{3}, P, \lambda\right)=-\left(\mathcal{L}_{u_{1}}+P \mathcal{L}_{u_{2}}+\mathcal{L}_{u_{3}}(\mathcal{U}+\mathcal{V} P)\right)\left(u_{1}, u_{2}, u_{3}, P, \lambda\right)
$$

teremos que os pontos parcialmente do tipo $D_{12}$ são definidos pelo sistema

$$
\left\{\begin{array}{l}
L_{r}\left(u_{1}, u_{2}, u_{3}, \lambda\right)=0 \\
M_{r}\left(u_{1}, u_{2}, u_{3}, \lambda\right)=0 \\
X_{4}\left(u_{1}, u_{2}, u_{3}, P, \lambda\right)=0 \\
\frac{\partial X_{4}}{\partial P}\left(u_{1}, u_{2}, u_{3}, P, \lambda\right)=0
\end{array} .\right.
$$

Desenvolvendo os cálculos vemos que

$$
\begin{aligned}
& \left.\operatorname{det}\left(\frac{\partial\left(L_{r}, M_{r}, X_{4}, \frac{\partial X_{4}}{\partial P}\right)}{\partial\left(u_{1}, u_{2}, u_{3}, \lambda\right)}\right)\right|_{u_{1}=u_{2}=u_{3}=\lambda=P=0}= \\
& =\frac{\partial d}{\partial \lambda}(0)\left(b^{2} \frac{\partial^{2} X_{4}}{\partial u_{3} \partial P}(0)-b q_{111} \frac{\partial^{2} X_{4}}{\partial u_{2} \partial P}(0)+\frac{\partial^{2} X_{4}}{\partial u_{1} \partial P}(0)\left(-b q_{201}+b q_{021}-c q_{111}\right)\right)
\end{aligned}
$$

Assim, supondo

$$
\frac{\partial d}{\partial \lambda}(0) \neq 0
$$

concluímos que o diagrama de bifurcação de um ponto do tipo $D_{1 h, p}^{1}$ é como na figura 6.8 e o diagrama de bifurcação de um ponto do tipo $D_{1 h, n}^{1}$ é como na figura 6.9. Além disso, o desdobramento transversal da $\alpha$ é equivalente à família

$$
\alpha\left(u_{1}, u_{2}, u_{3}, \lambda\right)=\left(u_{1}, u_{2}, u_{3}, h\left(u_{1}, u_{2}, u_{3}\right)+\frac{\lambda}{2} u_{1}^{2} u_{2}\right) .
$$


Diagramas de bifurcação dos pontos $D_{1}^{1}, D_{2}^{1}, D_{3}^{1}, D_{13}^{1}, D_{1 h, n}^{1}, D_{1 h, p}^{1}, D_{p}^{1}$ e $D_{c}^{1}$

\subsection{Diagramas de Bifurcação dos Pontos Parcialmente Umbílicos $D_{p}^{1}$ e $D_{c}^{1}$}

Seja $p$ um ponto parcialmente umbílico do tipo $D_{p}^{1}$ ou $D_{c}^{1}$ de uma imersão $\alpha: \mathbb{M}^{3} \rightarrow \mathbb{R}^{4}$. Escrevemos $\alpha$ como em (6.1) com $k=0$ e $h$ satisfazendo a condição $D_{p}^{1}$ ou $D_{c}^{1}$. Seja $\alpha_{\lambda}$ uma família a um parâmetro de imersões tal que $\alpha_{0}=\alpha$. Podemos escrever

$$
\alpha_{\lambda}\left(u_{1}, u_{2}, u_{3}\right)=\left(u_{1}, u_{2}, u_{3}, h_{\lambda}\left(u_{1}, u_{2}, u_{3}\right)\right)
$$

onde

$$
\begin{aligned}
h_{\lambda}\left(u_{1}, u_{2}, u_{3}, \lambda\right)= & a_{110}(\lambda) u_{1} u_{2}+a_{101}(\lambda) u_{1} u_{3}+a_{011}(\lambda) u_{2} u_{3}+\frac{k_{1}(\lambda)}{2} u_{1}^{2}+ \\
& +\frac{k_{2}(\lambda)}{2} u_{2}^{2}+\frac{k_{3}(\lambda)}{2} u_{3}^{2}+\frac{a(\lambda)}{6} u_{1}^{3}+\frac{b(\lambda)}{2} u_{1} u_{2}^{2}+\frac{d(\lambda)}{6} u_{1}^{2} u_{2}+ \\
& +\frac{c(\lambda)}{6} u_{2}^{3}+\frac{q_{003}(\lambda)}{6} u_{3}^{3}+\frac{q_{012}(\lambda)}{2} u_{2} u_{3}^{2}+q_{111}(\lambda) u_{1} u_{2} u_{3}+ \\
& +\frac{q_{021}(\lambda)}{2} u_{2}^{2} u_{3}+\frac{q_{102}(\lambda)}{2} u_{1} u_{3}^{2}+\frac{q_{201}(\lambda)}{2} u_{1}^{2} u_{3}+\frac{A(\lambda)}{24} u_{1}^{4}+ \\
& +\frac{B(\lambda)}{6} u_{1}^{3} u_{2}+\frac{C(\lambda)}{4} u_{1}^{2} u_{2}^{2}+\frac{D(\lambda)}{6} u_{1} u_{2}^{3}+\frac{E(\lambda)}{24} u_{2}^{4}+\frac{Q_{004}(\lambda)}{24} u_{3}^{4}+ \\
& +\frac{Q_{013}(\lambda)}{6} u_{3}^{3} u_{2}+\frac{Q_{103}(\lambda)}{6} u_{3}^{3} u_{1}+\frac{Q_{022}(\lambda)}{4} u_{2}^{2} u_{3}^{2}+\frac{Q_{202}(\lambda)}{4} u_{1}^{2} u_{3}^{2}+ \\
& +\frac{Q_{112}(\lambda)}{2} u_{3}^{2} u_{1} u_{2}+\frac{Q_{031}(\lambda)}{6} u_{3} u_{2}^{3}+\frac{Q_{301}(\lambda)}{6} u_{1}^{3} u_{3}+ \\
& +\frac{Q_{121}(\lambda)}{2} u_{3} u_{2}^{2} u_{1}+\frac{Q_{211}(\lambda)}{2} u_{3} u_{2} u_{1}^{2}+O(5)
\end{aligned}
$$


Diagramas de bifurcação dos pontos $D_{1}^{1}, D_{2}^{1}, D_{3}^{1}, D_{13}^{1}, D_{1 h, n}^{1}, D_{1 h, p}^{1}, D_{p}^{1}$ e $D_{c}^{1}$

Teorema 19 Seja 0 um ponto parcialmente umbilico do tipo $D_{p}^{1}$ de uma imersão $\alpha$ como em (6.1). O diagrama de bifurcação de um ponto deste tipo é como na figura 6.10 .

$$
\lambda<0
$$
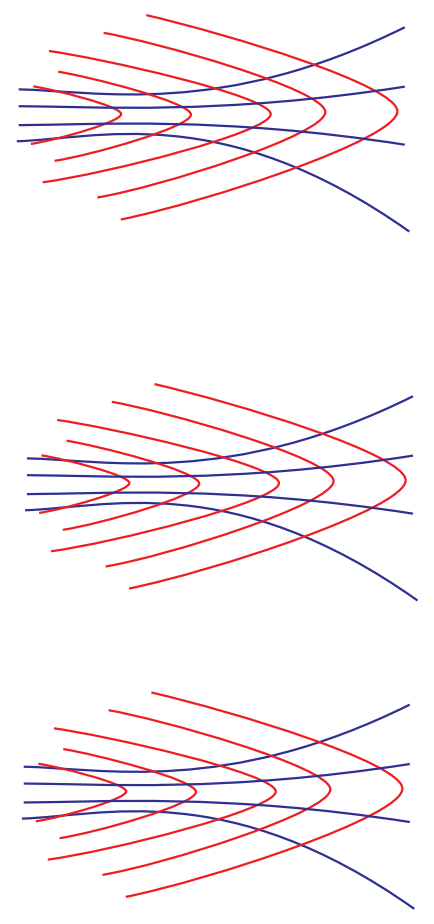

$\lambda=0$
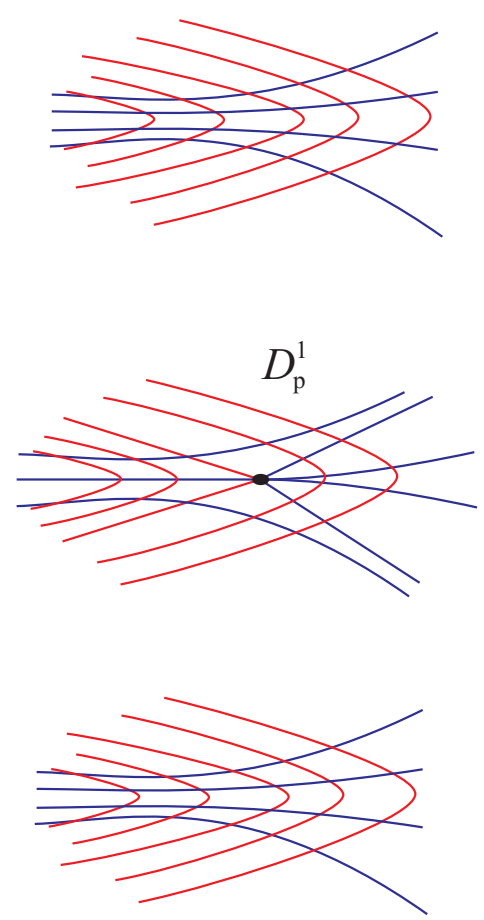

$\lambda>0$

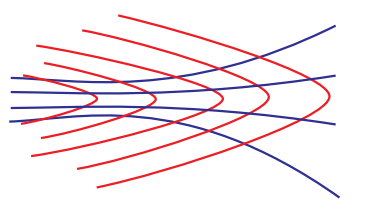

$D_{23}$

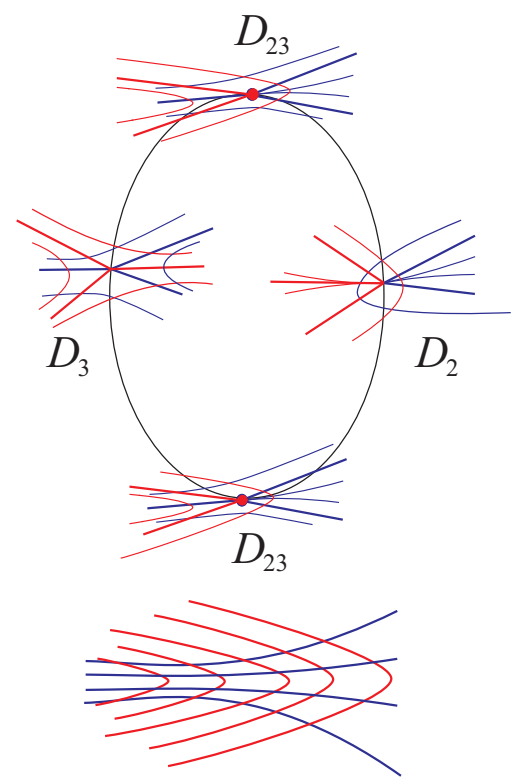

Figura 6.10: Bifurcação de uma linha parcialmente umbílica que contém um ponto do tipo $D_{p}^{1}$. 
Diagramas de bifurcação dos pontos $D_{1}^{1}, D_{2}^{1}, D_{3}^{1}, D_{13}^{1}, D_{1 h, n}^{1}, D_{1 h, p}^{1}, D_{p}^{1}$ e $D_{c}^{1}$

Teorema 20 Seja 0 um ponto parcialmente umbílico do tipo $D_{c}^{1}$ de uma imersão $\alpha$ como em 6.1. O diagrama de bifurcação de um ponto deste tipo é como na figura 6.13.
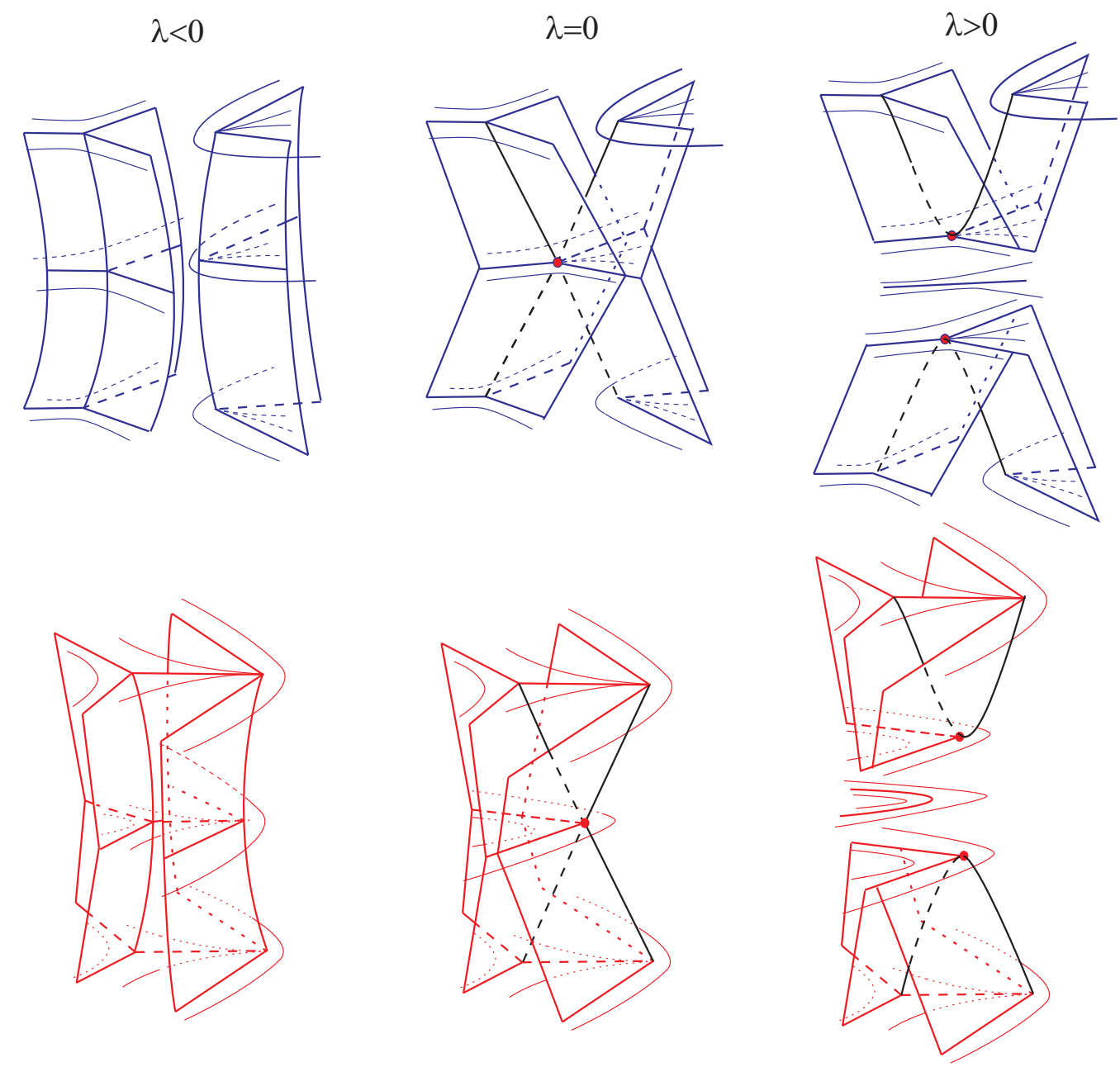

Figura 6.11: Bifurcação de uma linha parcialmente umbílica que contém um ponto do tipo $D_{c}^{1}$. 
Diagramas de bifurcação dos pontos $D_{1}^{1}, D_{2}^{1}, D_{3}^{1}, D_{13}^{1}, D_{1 h, n}^{1}, D_{1 h, p}^{1}, D_{p}^{1}$ e $D_{c}^{1}$

Demonstração dos teoremas 19 e 20: A equação diferencial das linhas de curvatura é dada por

$$
\mathcal{L}\left(u_{1}, u_{2}, u_{3}, P, \lambda\right)=L_{r}\left(u_{1}, u_{2}, u_{3}, \lambda\right) P^{2}+M_{r}\left(u_{1}, u_{2}, u_{3}, \lambda\right) P+N_{r}\left(u_{1}, u_{2}, u_{3}, \lambda\right)
$$

onde

$$
\begin{aligned}
L_{r} & =-a_{110}(\lambda)+\left(-b(\lambda)+p_{010}(\lambda) a_{011}(\lambda)+q_{010}(\lambda) a_{101}(\lambda)\right) u_{2}+ \\
& +\left(-q_{111}(\lambda)+p_{001}(\lambda) a_{011}(\lambda)+q_{001}(\lambda) a_{101}(\lambda)\right) u_{3}+ \\
& +\left(-d(\lambda)+p_{100}(\lambda) a_{011}(\lambda)+q_{100}(\lambda) a_{101}(\lambda)\right) u_{1}+O(2)
\end{aligned}
$$

$$
\begin{aligned}
M_{r} & =a_{020}(\lambda)-a_{200}(\lambda)+\left(2 a_{101}(\lambda) p_{010}(\lambda)-d(\lambda)-2 a_{011}(\lambda) q_{010}(\lambda)+c(\lambda)\right) u_{2}+ \\
& +\left(-2 a_{011}(\lambda) q_{001}(\lambda)+q_{021}(\lambda)-q_{201}(\lambda)+2 a_{101}(\lambda) p_{001}(\lambda)\right) u_{3}+ \\
& +\left(2 a_{101}(\lambda) p_{100}(\lambda)-a(\lambda)(\lambda)-2 a_{011}(\lambda) q_{100}(\lambda)+b(\lambda)\right) u_{1}+O(2)
\end{aligned}
$$

$$
\begin{aligned}
N_{r} & =a_{110}(\lambda)-\left(-b(\lambda)+p_{010}(\lambda) a_{011}(\lambda)+q_{010}(\lambda) a_{101}(\lambda)\right) u_{2}- \\
& -\left(-q_{111}(\lambda)+p_{001}(\lambda) a_{011}(\lambda)+q_{001}(\lambda) a_{101}(\lambda)\right) u_{3}- \\
& -\left(-d(\lambda)+p_{100}(\lambda) a_{011}(\lambda)+q_{100}(\lambda) a_{101}(\lambda)\right) u_{1}+O(2)
\end{aligned}
$$

onde

$$
\begin{gathered}
p_{100}(\lambda)=-2 \frac{-2 q_{201}(\lambda) k_{2}(\lambda)+q_{201}(\lambda) k_{3}(\lambda)+2 q_{111}(\lambda) a_{110}(\lambda)}{4\left(a_{110}(\lambda)\right)^{2}-\left(2 k_{2}(\lambda)-k_{3}(\lambda)\right)\left(2 k_{1}(\lambda)-k_{3}(\lambda)\right)} \\
p_{010}(\lambda)=-2 \frac{-4 q_{111}(\lambda)(\lambda) k_{2}(\lambda)+2 q_{111}(\lambda) k_{3}(\lambda)+4 a_{110}(\lambda) q_{021}(\lambda)}{4\left(a_{110}(\lambda)\right)^{2}-\left(2 k_{2}(\lambda)-k_{3}(\lambda)\right)\left(2 k_{1}(\lambda)-k_{3}(\lambda)\right)}, \\
p_{001}(\lambda)=-2 \frac{-4 q_{102}(\lambda) k_{2}(\lambda)+2 q_{102}(\lambda) k_{3}(\lambda)+4 a_{110}(\lambda) q_{012}(\lambda)}{4\left(a_{110}(\lambda)\right)^{2}-\left(2 k_{2}(\lambda)-k_{3}(\lambda)\right)\left(2 k_{1}(\lambda)-k_{3}(\lambda)\right)} \\
q_{100}(\lambda)=-2 \frac{4 a_{110}(\lambda) q_{201}(\lambda)+2 q_{111}(\lambda) k_{3}(\lambda)-4 q_{111}(\lambda) k_{1}(\lambda)}{4\left(a_{110}(\lambda)\right)^{2}-\left(2 k_{2}(\lambda)-k_{3}(\lambda)\right)\left(2 k_{1}(\lambda)-k_{3}(\lambda)\right)} \\
q_{010}(\lambda)=-2 \frac{4 q_{111}(\lambda) a_{110}(\lambda)+2 q_{021}(\lambda) k_{3}(\lambda)-4 k_{1}(\lambda) q_{021}(\lambda)}{4\left(a_{110}(\lambda)\right)^{2}-\left(2 k_{2}(\lambda)-k_{3}(\lambda)\right)\left(2 k_{1}(\lambda)-k_{3}(\lambda)\right)} \\
q_{001}(\lambda)=-2 \frac{4 q_{102}(\lambda) a_{110}(\lambda)-4 k_{1}(\lambda) q_{012}(\lambda)+2 q_{012}(\lambda) k_{3}(\lambda)}{4\left(a_{110}(\lambda)\right)^{2}-\left(2 k_{2}(\lambda)-k_{3}(\lambda)\right)\left(2 k_{1}(\lambda)-k_{3}(\lambda)\right)} .
\end{gathered}
$$

Seja

$$
\mathcal{D}=\frac{\partial L}{\partial u_{1}} \frac{\partial M}{\partial u_{2}}-\frac{\partial M}{\partial u_{1}} \frac{\partial L}{\partial u_{2}}
$$


Diagramas de bifurcação dos pontos $D_{1}^{1}, D_{2}^{1}, D_{3}^{1}, D_{13}^{1}, D_{1 h, n}^{1}, D_{1 h, p}^{1}, D_{p}^{1}$ e $D_{c}^{1}$

O conjunto dos pontos parcialmetne umbílicos do tipo $D_{23}$ é dado por

$$
\left\{\begin{array}{l}
L_{r}\left(u_{1}, u_{2}, u_{3}, \lambda\right)=0 \\
M_{r}\left(u_{1}, u_{2}, u_{3}, \lambda\right)=0 \\
\mathcal{D}\left(u_{1}, u_{2}, u_{3}, \lambda\right)=0
\end{array}\right.
$$

Usando (6.22), (6.23) e a condição de que a origem é um ponto parcialmente umbílico do tipo $D_{p}^{1}$ ou $D_{c}^{1}$ de $\alpha_{0}$, teremos que

$$
\left(\left.\frac{\partial\left(L_{r}, M_{r}, \mathcal{D}\right)}{\partial\left(u_{1}, u_{2}, u_{3}, \lambda\right)}\right|_{u_{1}=0=u_{2}=u_{3}=\lambda}\right)=\left(\begin{array}{cccc}
0 & 0 & 0 & \frac{\partial a_{110}}{\partial \lambda}(0) \\
-a(0) & c(0) & q_{021}(0)-q_{201}(0) & \frac{\partial a_{020} 0}{\partial \lambda}(0)-\frac{\partial a_{200}}{\partial \lambda}(0) \\
a_{1} & a_{2} & a_{3} & a_{4}
\end{array}\right)
$$

onde $a_{1}=q_{201}(0) q_{021}(0) a(0)-c(0) B(0)-C(0) a(0), a_{2}=c(0) q_{201}(0) q_{021}(0)-D(0) a(0)-$ $c(0) C(0), a_{3}=c(0) q_{201}(0) q_{012}(0)+q_{102}(0) q_{021}(0) a(0)-c(0) Q_{211}(0)-Q_{121}(0) a(0), a_{4}=$ $-b^{\prime}(0) a(0)$. Supondo $\frac{\partial a_{110}}{\partial \lambda}(0) \neq 0$ teremos que (6.25) forma uma curva regular no espaço das variáveis $u_{1}, u_{2}, u_{3}$ e $\lambda$.

Assim, concluímos que o diagrama de bifurcação de um ponto do tipo $D_{p}^{1}$ é como na figura 6.10 e o diagrama de bifurcação de um ponto do tipo $D_{c}^{1}$ é como na figura 6.13. Além disso, o desdobramento transversal da $\alpha$ é equivalente à família

$$
\alpha\left(u_{1}, u_{2}, u_{3}, \lambda\right)=\left(u_{1}, u_{2}, u_{3}, h\left(u_{1}, u_{2}, u_{3}\right)+\lambda u_{1} u_{2}\right) .
$$


Diagramas de bifurcação dos pontos $D_{1}^{1}, D_{2}^{1}, D_{3}^{1}, D_{13}^{1}, D_{1 h, n}^{1}, D_{1 h, p}^{1}, D_{p}^{1}$ e $D_{c}^{1}$

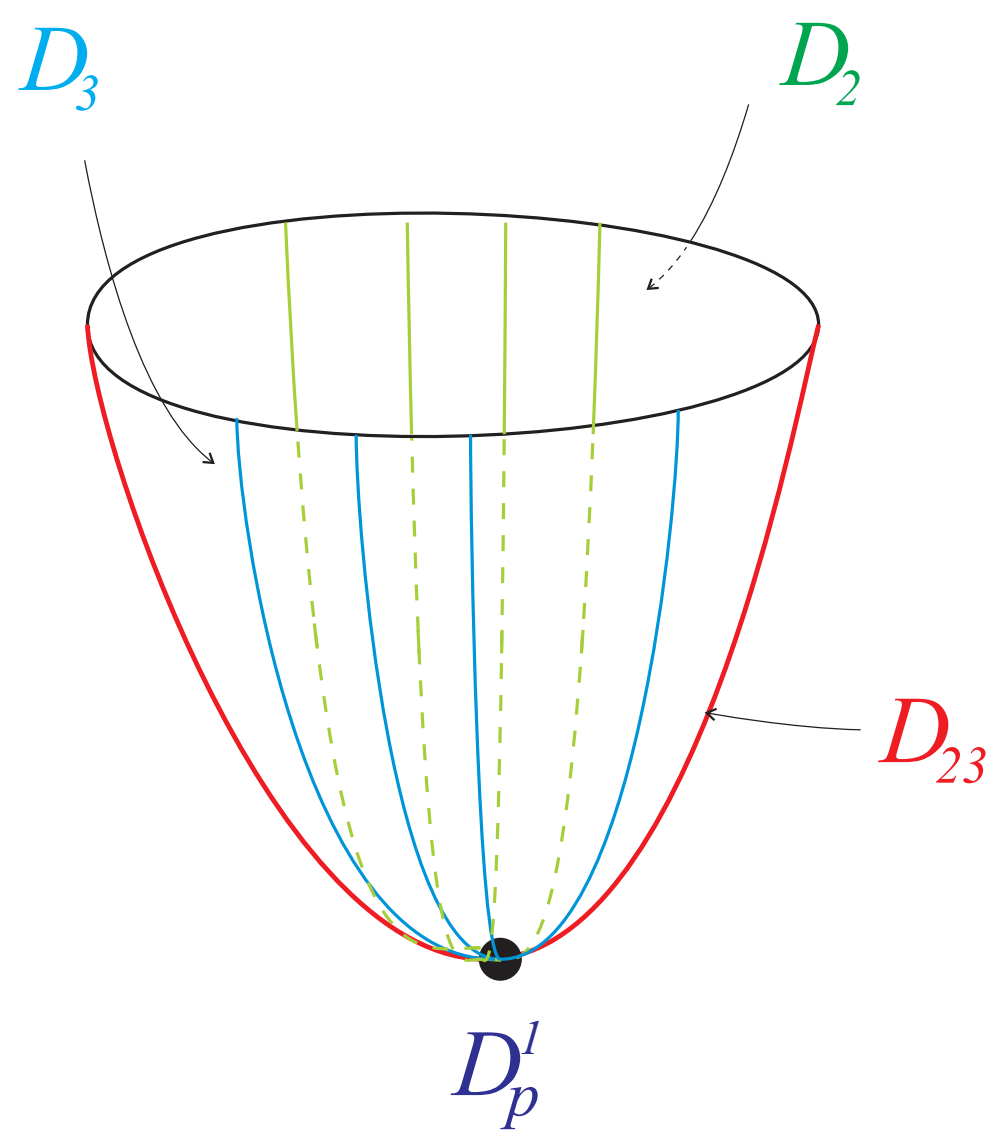

Figura 6.12: Conjunto parcialmente umbílico da família $\alpha_{\lambda}$ nas proximidades de um ponto do tipo $D_{p}^{1}$. 
Diagramas de bifurcação dos pontos $D_{1}^{1}, D_{2}^{1}, D_{3}^{1}, D_{13}^{1}, D_{1 h, n}^{1}, D_{1 h, p}^{1}, D_{p}^{1}$ e $D_{c}^{1}$

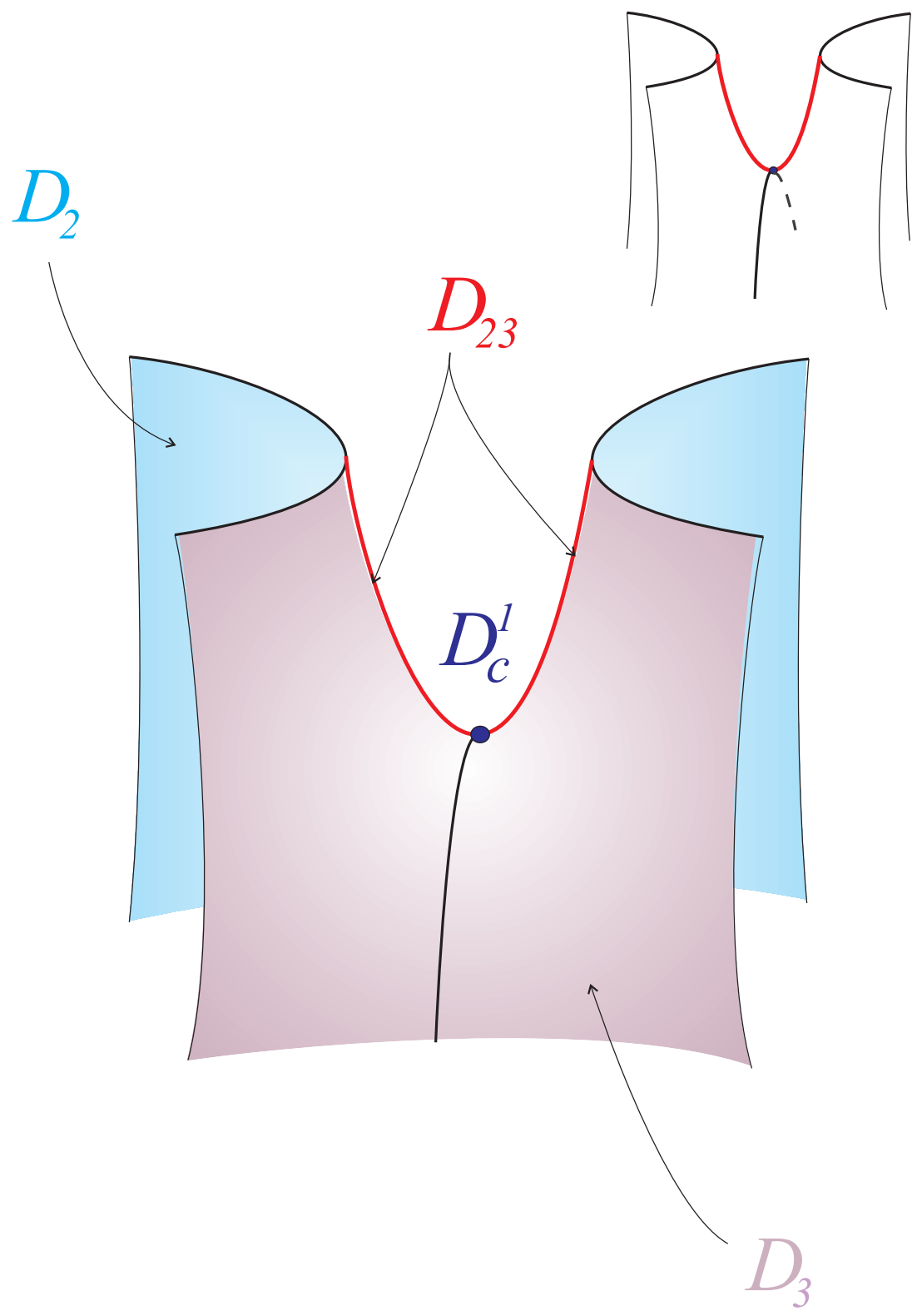

Figura 6.13: Conjunto parcialmente umbílico da família $\alpha_{\lambda}$ nas proximidades de um ponto do tipo $D_{c}^{1}$. 


\section{Capítulo 7}

\section{Conclusões}

Neste trabalho estudamos o comportamento das folheações principais, numa vizinhança dos pontos parcialmente umbílicos, e seus diagramas de bifurcação em famílias genéricas de hipersuperfícies imersas em $\mathbb{R}^{4}$ dependendo de um parâmetro. Isto da continuidade ao estudo dos pontos parcialmente umbílicos genéricos feito por Garcia em [6] e [7].

Usando o levantamento de Lie-Cartan definido na seção 1.2 do capítulo 1, estudamos nos teoremas 5, 6, 7, 8, 9, 10 e 11 o comportamento das folheações principais numa vizinhança de um ponto parcialmente umbílico do tipo $D_{1}^{1}, D_{2}^{1}, D_{3}^{1}, D_{13}^{1}, D_{1 h, n}^{1}$, $D_{1 h, p}^{1}, D_{p}^{1}$ e $D_{c}^{1}$. ( Ver definições $8,9,10,11,4.1$ e 4.2 ).

Com o teorema 12, no capítulo 5 , concluimos que as singularidades das folheações principais que aparecem genericamente em famílias a 1 parâmetro de hipersuperfícies são os pontos parcialmente umbílicos dos tipos $D_{1}^{1}, D_{2}^{1}, D_{3}^{1}, D_{13}^{1}, D_{1 h, n}^{1}, D_{1 h, p}^{1}, D_{p}^{1}, D_{c}^{1}$. Os diagramas de bifurcação dos pontos $D_{1}^{1}, D_{2}^{1}, D_{3}^{1}, D_{13}^{1}, D_{1 h, n}^{1}, D_{1 h, p}^{1}, D_{p}^{1}$ e $D_{c}^{1}$ foram obtidos nos teoremas 13,14,15,16,17,18, 19 e 20 no capítulo 6 .

Esta conclusão sintetiza a contribuição deste trabalho. 


\section{Bibliografia}

[1] J. Bruce e D. Fidal, On Binary differential equations and umbilics, Proc. Royal Soc. Edinburgh, 111A (1987)

[2] G. Darboux, Leçons Sur la Théorie des Surfaces, vol. IV. Sur la forme des lignes de Courbure dans la voisinage d'un ombilic, Note 7 , Paris: Gauthier Villars.

[3] F. Dumortier, J.Llibre, J. C. Artés Qualitative Theory of Planar Differential Systems, Springer, 2006.

[4] E. Feldman, On parabolic and umbilic points on hypersurfaces, Trans. Amer Math. Soc., 127, (1967)

[5] N. Fenichel, Persistence and Smootheness of Invariant Manifolds of Flows, Indiana University Math, J. 21,(1971 - 1972).

[6] R. Garcia, Linhas de curvatura de hipersuperfícies imersas no espaço $\mathbb{R}^{4}$, PréPublicação-IMPA, (Thesis), Série F, 27, (1989).

[7] R. Garcia, Principal Curvature lines near Darbouxian partially umbilic points of hypersurfaces immersed in $\mathbb{R}^{4}$, Comp. and Appl. Math., 20, (2001), pp. 121148.

[8] R. Garcia, C. Gutierrez e J. Sotomayor, Birfucations of Umbilical Points and Related Principal Cycles, Jour. Dyn. and Diff. Equations, 16 (2004), pp. 321346.

[9] R. Garcia e J. Sotomayor, Codimension Two Umbilics Points on Surfaces Immersed in $\mathbb{R}^{3}$, Discrete and Continuous Dynamical Systems, 17, No. 2, (2007), pp. 293-308.

[10] R. Garcia e J. Sotomayor, Principal lines near principal cycles, Annals of Global Analysis and Geometry, 10 (1992), pp. 275-289. 
[11] R. Garcia e J. Sotomayor, Differential equations of classical geometry a qualitative

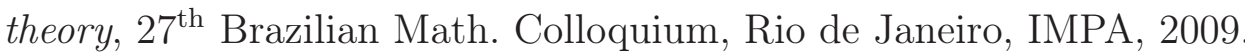

[12] C. Gutierrez e J. Sotomayor, Structural Stable Configurations of lines of Principal Curvature, Asterisque, 98-99, (1982), pp. 185-215.

[13] C. Gutierrez e J. Sotomayor, Periodic lines of curvature bifurcating from Darbouxian umbilical connections, Springer Lect. Notes in Math., (1990), 1455, pp. 196-229.

[14] C. Gutierrez e J. Sotomayor, Closed Principal Lines and Bifurcations, Bol. Soc. Bras. Mat., 17 , (1986), pp. 1-19.

[15] N. J. Hicks, Notes on Differential Geometry, Van Nostrand Reinhold Company, 1965 .

[16] M. Hirsh, C. Pugh e M. Shub, Invariant Manifolds, Lectures Notes in Math, 583, (1977).

[17] H. T. Levine, Singularities of Diferentiable Mappings, Lect. Notes in Math, 192 (1971), pp. 1-89.

[18] A. Remizov, Multidimensional Poincaré construction and singularities of lifted fields for implicit differential equations, Journal of Mathematical Sciences, 151, No. 6, (2008), pp. 3561-3602. 


\section{Índice}

Campo de Lie-Cartan, 23, 28

Campo de linhas principais, 16

Carta de Monge, 17

Carta de Monge reduzida, 17

Conjunto normalmente hiperbólico, 84

Conjunto parcialmente umbílico, 16

Conjunto umbílico, 16

Curva parcialmente umbílica Darbouxiana do tipo $D_{i}(i=1,2,3), 19$

Curvaturas principais, 16

Direção regular, 23

Direções principais, 16

Folheações principais, 21

Hipersuperfície de Lie-Cartan, 27

Imersão, 15

Levantamento de Lie-Cartan, 23

Linhas de curvatura principal, 21

Linhas principais, 21

Normalmente hiperbólico do tipo atrator, 84

Normalmente hiperbólico do tipo repulsor, 84

Normalmente hiperbólico do tipo sela, 84

Plano umbílico, 26

Ponto parcialmente umbílico $D_{13}^{1}, 105$
Ponto parcialmente umbílico $D_{1 h, p}^{1}$ e $D_{1 h, n}^{1}$, 123

Ponto parcialmente umbílico $D_{2}^{1}$ e $D_{3}^{1}, 98$

Ponto parcialmente umbílico $D_{c}^{1}, 133$

Ponto parcialmente umbílico $D_{p}^{1}, 133$

Ponto parcialmente umbílico bi-regular, 26

Ponto parcialmente umbílico crítico, 26

Ponto parcialmente umbílico Darbouxiano, 17

Ponto parcialmente umbílico do tipo $D_{1}^{1}$, 88

Ponto parcialmente umbílico regular, 26

Ponto parcialmente umbílico regular-tangente, 26

Ponto parcialmente umbílico semi-Darbouxiano do tipo $D_{12}, 20$

Ponto parcialmente umbílico semi-Darbouxiano do tipo $D_{23}, 20$

Pontos parcialmente umbílicos, 16

Pontos umbílicos, 16

Separatriz Parcialmente Umbílica de $p, 40$

Setor, 40

Setor parabólico, 40, 41

Singularidades, 16

Superfície Separatriz Parcialmente Umbílica de $c, 40$

Variedade do tipo sela-nó, 68 
Variedade estável, 84 